Supporting Information for

\title{
Asymmetric Hydrogenation of Dibenzo[c,e]azepine Derivatives with Chiral Cationic Ruthenium Diamine Catalysts
}

\author{
Shanshan Zhang, ${ }^{\dagger}$ Fei Chen, ${ }^{\dagger}{ }^{\dagger}$ Yan-Mei He, ${ }^{\dagger}$ and Qing-Hua Fan $*^{\dagger}$ \\ ${ }^{\dagger}$ Beijing National Laboratory for Molecular Sciences, CAS Key Laboratory of \\ Molecular Recognition and Function, Institute of Chemistry, Chinese Academy of \\ Sciences (CAS), and University of Chinese Academy of Sciences, Beijing 100190, P. \\ R. China \\ E-mail: fanqh@iccas.ac.cn; chenfei211@iccas.ac.cn
}

\section{Contents}

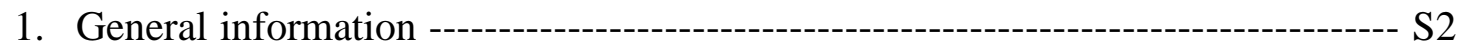

2. Optimization of conditions for asymmetric hydrogenation ----------------- S2

3. General procedure for the synthesis of dibenzo[c,e]azepines ---------------------S6

4. General procedure for asymmetric hydrogenation of dibenzo[c,e]azepines -S12

5. General procedure for asymmetric reductive amination --------------------------S22

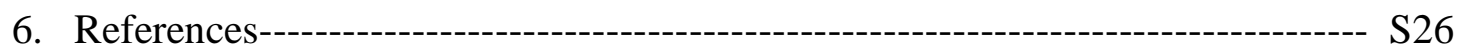

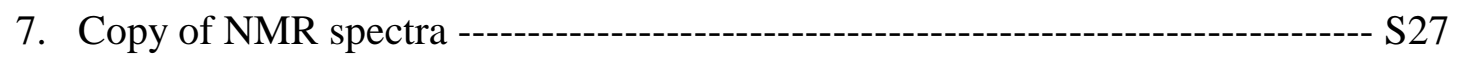

8. Copy of HPLC spectra ----------------------------------------------------------------- S72 


\section{General information}

Unless otherwise noted, all experiments were carried out under an atmosphere of nitrogen using standard Schlenk techniques or in a nitrogen-filled glovebox. ${ }^{1} \mathrm{H}$ NMR and ${ }^{13} \mathrm{C}$ NMR spectra were recorded on a Bruker Model Avance DMX 400 Spectrometer $\left({ }^{1} \mathrm{H} 400 \mathrm{MHz}\right.$ and ${ }^{13} \mathrm{C} 100 \mathrm{MHz}$, respectively) or Bruker Model Avance DMX 500 Spectrometer $\left({ }^{1} \mathrm{H} 500 \mathrm{MHz}\right.$ and ${ }^{13} \mathrm{C} 125 \mathrm{MHz}$, respectively). Chemical shifts $(\delta)$ were given in ppm and were referenced to residual solvent or TMS peaks. Optical rotations were measured with Rudolph Autopl VI polarimeter. High resolution MS (P-ESI HRMS) were obtained on Bruker Apex IV FTMS spectrometer or Thermo Fisher Q Exactive Mass Spectrometer. HPLC analyses were performed on a Varian Prostar 210 liquid chromatograph. All organic solvents were dried using standard, published methods and were distilled before use. All other chemicals were used as received from Aldrich or Acros without further purification. The catalysts were prepared according to the published methods. ${ }^{1}$ Dibenzo[c,e]azepines were synthesized according to modified literature methods. ${ }^{2}$

\section{Optimization of conditions for asymmetric hydrogenation}

Table S1: Optimization of conditions for asymmetric hydrogenation of $\mathbf{1 a}^{a}$

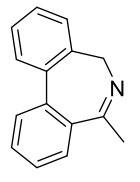

1a

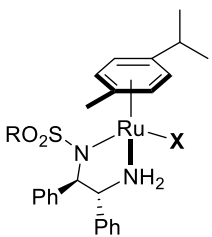

$2.0 \mathrm{~mol} \%(R, R)-3-6$

50 atm $\mathrm{H}_{2}, 50^{\circ} \mathrm{C}$, solvent $22 \mathrm{~h}, 1.1$ equiv $(\mathrm{Boc})_{2} \mathrm{O}$

$(R, R)-3: \mathrm{R}=\mathrm{CH}_{3}$ $(R, R)-4: \mathrm{R}=4-\mathrm{CH}_{3} \mathrm{C}_{6} \mathrm{H}_{4}$ $(R, R)-5: \mathrm{R}=\mathrm{CF}_{3}$ $(R, R)-6: \mathrm{R}=\mathrm{N}\left(\mathrm{CH}_{2}\right)_{5}$

$\mathbf{X}=\mathrm{OMs}(\mathbf{a}) ; \mathrm{OTf}(\mathbf{b}) ; \mathrm{BF}_{4}(\mathbf{c})$; $\mathrm{PF}_{6}(\mathbf{d}) ; \mathrm{SbF}_{6}(\mathbf{e}) ; \operatorname{BArF}(\mathbf{f})$ 


\begin{tabular}{|c|c|c|c|c|c|c|}
\hline entry & catalyst & {$[\mathrm{X}]^{-}$} & solvent & $\begin{array}{l}\mathrm{H}_{2}(\mathrm{~atm}) \\
\text { temp }\left({ }^{\mathrm{o}} \mathrm{c}\right)\end{array}$ & conv. $(\%)^{b}$ & ee $(\%)^{c}$ \\
\hline 1 & $(R, R)-\mathbf{3 a}$ & OMs & DCM & $50 ; 50$ & $>99$ & 3 \\
\hline 2 & $(R, R)-\mathbf{3 b}$ & OTf & DCM & $50 ; 50$ & $>99$ & 12 \\
\hline 3 & $(R, R)-3 \mathbf{c}$ & $\mathrm{BF}_{4}$ & DCM & $50 ; 50$ & $>99$ & 26 \\
\hline 4 & $(R, R)-\mathbf{3 d}$ & $\mathrm{PF}_{6}$ & DCM & $50 ; 50$ & $>99$ & 50 \\
\hline 5 & $(R, R)-\mathbf{3 e}$ & $\mathrm{SbF}_{6}$ & DCM & $50 ; 50$ & $>99$ & 59 \\
\hline 6 & $(R, R)-\mathbf{3 f}$ & $\mathrm{BArF}$ & DCM & $50 ; 50$ & $>99$ & 79 \\
\hline $7^{d}$ & $(R, R)-\mathbf{3 f}$ & $\mathrm{BArF}$ & DCM & $50 ; 50$ & 68 & 58 \\
\hline 8 & $(R, R)-\mathbf{3 f}$ & $\mathrm{BArF}$ & toluene & $50 ; 50$ & $>99$ & 83 \\
\hline 9 & $(R, R)-\mathbf{3 f}$ & $\mathrm{BArF}$ & DCE & $50 ; 50$ & $>99$ & 45 \\
\hline 10 & $(R, R)-\mathbf{3 f}$ & $\mathrm{BArF}$ & THF & $50 ; 50$ & $>99$ & 3 \\
\hline 11 & $(R, R)-\mathbf{3 f}$ & $\mathrm{BArF}$ & $\mathrm{Et}_{2} \mathrm{O}$ & $50 ; 50$ & $>99$ & 47 \\
\hline 12 & $(R, R)-\mathbf{3 f}$ & BArF & $\mathrm{MeOH}$ & $50 ; 50$ & $>99$ & -18 \\
\hline 13 & $(R, R)-\mathbf{3 f}$ & $\mathrm{BArF}$ & ${ }^{i} \mathrm{PrOH}$ & $50 ; 50$ & $>99$ & 34 \\
\hline 14 & $(R, R)-\mathbf{4 f}$ & $\mathrm{BArF}$ & toluene & $50 ; 50$ & $>99$ & 82 \\
\hline 15 & $(R, R)-\mathbf{5 f}$ & $\mathrm{BArF}$ & toluene & $50 ; 50$ & $>99$ & 73 \\
\hline 16 & $(R, R)-\mathbf{6 f}$ & $\mathrm{BArF}$ & toluene & $50 ; 50$ & $>99$ & 91 \\
\hline 17 & $(R, R)-\mathbf{6 f}$ & $\mathrm{BArF}$ & toluene & $50 ; 25$ & $>99$ & 93 \\
\hline 18 & $(R, R)-\mathbf{6} \mathbf{f}$ & $\mathrm{BArF}$ & toluene & $50 ; 80$ & $>99$ & 88 \\
\hline 19 & $(\boldsymbol{R}, \boldsymbol{R})-\mathbf{6 f}$ & BArF & toluene & $30 ; 25$ & $>99$ & 94 \\
\hline 20 & $(R, R)-\mathbf{6 f}$ & $\mathrm{BArF}$ & toluene & $1 ; 25$ & 34 & 87 \\
\hline $21^{e}$ & $(R, R)-\mathbf{6 f}$ & $\mathrm{BArF}$ & toluene & $30 ; 25$ & 65 & 76 \\
\hline
\end{tabular}

${ }^{a}$ Reaction conditions: 1a $(0.1 \mathrm{mmol})$ in solvent $(1.0 \mathrm{~mL}),(R, R)-\mathrm{Ru}$ catalyst $(2.0$ mol \%), (Boc) $)_{2} \mathrm{O}$ (1.1 equiv), $\mathrm{H}_{2}(50 \mathrm{~atm})$, stirred at $50{ }^{\circ} \mathrm{C}$ for $22 \mathrm{~h} .{ }^{b}$ The conversions were determined by ${ }^{1} \mathrm{H}$ NMR spectroscopy of the crude reaction mixture. ${ }^{c}$ The enantiomeric excesses were determined by HPLC with a chiral IC column. ${ }^{d}$ Without (Boc) $)_{2} \mathrm{O} .{ }^{e}$ Ru-catalyst $(1.0 \mathrm{~mol} \%)$. 
Table S2: Optimization of conditions for asymmetric hydrogenation of $\mathbf{1} \mathbf{m}^{a}$
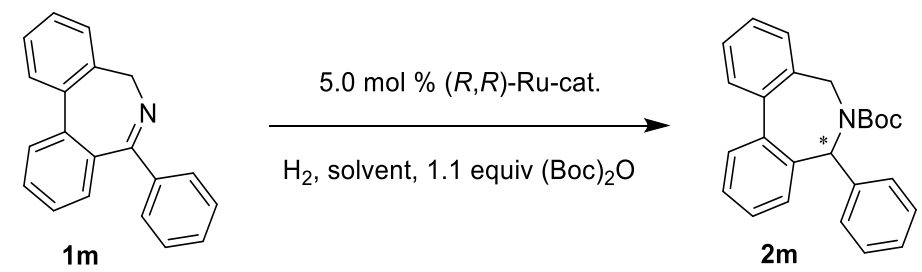

\begin{tabular}{|c|c|c|c|c|c|c|}
\hline entry & catalyst & {$[\mathrm{X}]^{-}$} & solvent & $\begin{array}{l}\mathrm{H}_{2}(\mathrm{~atm}) \\
\text { temp }\left({ }^{\mathrm{o}} \mathrm{c}\right)\end{array}$ & conv. $(\%)^{b}$ & ee $(\%)^{c}$ \\
\hline 1 & $(R, R)-\mathbf{3 a}$ & OMs & $\mathrm{Et}_{2} \mathrm{O}$ & $50 ; 50$ & 10 & 22 \\
\hline 2 & $(R, R)-\mathbf{3 b}$ & OTf & $\mathrm{Et}_{2} \mathrm{O}$ & $50 ; 50$ & 17 & 15 \\
\hline 3 & $(R, R)-3 \mathbf{c}$ & $\mathrm{BF}_{4}$ & $\mathrm{Et}_{2} \mathrm{O}$ & $50 ; 50$ & 20 & 38 \\
\hline 4 & $(R, R)-\mathbf{3 d}$ & $\mathrm{PF}_{6}$ & $\mathrm{Et}_{2} \mathrm{O}$ & $50 ; 50$ & 16 & 31 \\
\hline 5 & $(R, R)-\mathbf{3 e}$ & $\mathrm{SbF}_{6}$ & $\mathrm{Et}_{2} \mathrm{O}$ & $50 ; 50$ & 15 & 24 \\
\hline 6 & $(R, R)-\mathbf{3 f}$ & BArF & $\mathrm{Et}_{2} \mathrm{O}$ & $50 ; 50$ & 38 & 40 \\
\hline 7 & $(R, R)-\mathbf{3 f}$ & BArF & toluene & $50 ; 50$ & 17 & 10 \\
\hline 8 & $(R, R)-\mathbf{3 f}$ & BArF & DCE & $50 ; 50$ & 33 & 3 \\
\hline 9 & $(R, R)-\mathbf{3 f}$ & BArF & DCM & $50 ; 50$ & 41 & 2 \\
\hline 10 & $(R, R)-\mathbf{3 f}$ & $\mathrm{BArF}$ & THF & $50 ; 50$ & 35 & 8 \\
\hline 11 & $(R, R)-\mathbf{3 f}$ & $\mathrm{BArF}$ & $\mathrm{MeOH}$ & $50 ; 50$ & 42 & 2 \\
\hline 12 & $(R, R)-\mathbf{3 f}$ & $\mathrm{BArF}$ & ${ }^{i} \mathrm{PrOH}$ & $50 ; 50$ & 19 & 16 \\
\hline $13^{d}$ & $(R, R)-\mathbf{3 f}$ & $\mathrm{BArF}$ & $\mathrm{Et}_{2} \mathrm{O}$ & $50 ; 50$ & $>99$ & 40 \\
\hline $14^{d}$ & $(R, R)-\mathbf{6 f}$ & $\mathrm{BArF}$ & $\mathrm{Et}_{2} \mathrm{O}$ & $50 ; 50$ & $>99$ & -4 \\
\hline $15^{d}$ & $(\boldsymbol{R}, \boldsymbol{R})-\mathbf{5 f}$ & BArF & $\mathbf{E t}_{2} \mathbf{O}$ & $50 ; 50$ & $>99$ & 80 \\
\hline
\end{tabular}

${ }^{a}$ Reaction conditions: $1 \mathrm{~m}(0.1 \mathrm{mmol})$ in solvent $(1 \mathrm{~mL}),(R, R)-\mathrm{Ru}$ catalyst $(5.0$ mol \%), (Boc) $)_{2} \mathrm{O}$ (1.1 equiv), $\mathrm{H}_{2}(50 \mathrm{~atm})$, stirred at $50{ }^{\circ} \mathrm{C}$ for $22 \mathrm{~h} .{ }^{b}$ The conversions were determined by ${ }^{1} \mathrm{H}$ NMR spectroscopy of the crude reaction mixture. ${ }^{c}$ The enantiomeric excesses were determined by HPLC with a chiral IC column. ${ }^{d}(R, R)-\mathrm{Ru}$ catalyst $(10.0 \mathrm{~mol} \%)$. 
Table S3: Optimization of conditions for one-pot synthesis of chiral 6,7-dihydro-5H-dibenz $[c, e]$ azepines via two-step reductive amination ${ }^{a}$

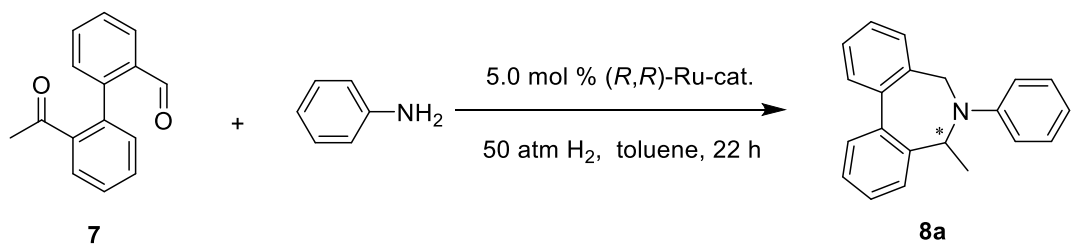

\begin{tabular}{ccccccc}
\hline entry & catalyst & {$[\mathrm{X}]^{-}$} & additive & temp $\left({ }^{\circ} \mathrm{c}\right)$ & conv. $(\%)^{b}$ & ee $(\%)^{c}$ \\
\hline 1 & $(R, R)-\mathbf{4 b}$ & OTf & none & 50 & 62 & 77 \\
2 & $(R, R)-\mathbf{4 b}$ & OTf & $4 \AA \mathrm{MS}$ & 50 & 78 & 70 \\
3 & $(R, R)-\mathbf{4 b}$ & OTf & TfOH & 50 & 64 & 78 \\
$\mathbf{4}$ & $(\boldsymbol{R}, \boldsymbol{R})-\mathbf{4 b}$ & OTf & $\mathbf{4} \AA \mathrm{MS} / \mathrm{TfOH}$ & $\mathbf{5 0}$ & $\mathbf{9 8}$ & $\mathbf{7 7}$ \\
5 & $(R, R)-\mathbf{4 b}$ & OTf & $4 \AA \mathrm{MS} / \mathrm{TfOH}$ & 25 & 57 & 85 \\
6 & $(R, R)-\mathbf{4 f}$ & BArF & $4 \AA \mathrm{MS} / \mathrm{TfOH}$ & 50 & 96 & 54 \\
7 & $(R, R)-\mathbf{3 b}$ & OTf & $4 \AA \mathrm{MS} / \mathrm{TfOH}$ & 50 & 61 & 72 \\
8 & $(R, R)-\mathbf{5 b}$ & OTf & $4 \AA \mathrm{MS} / \mathrm{TfOH}$ & 50 & 98 & 63 \\
9 & $(R, R)-\mathbf{6 b}$ & OTf & $4 \AA \mathrm{MS} / \mathrm{TfOH}$ & 50 & 95 & 71 \\
\hline
\end{tabular}

${ }^{a}$ Reaction conditions: $7(0.1 \mathrm{mmol})$ in toluene $(1 \mathrm{~mL})$, aniline $(0.1 \mathrm{mmol}),(R, R)-\mathrm{Ru}$

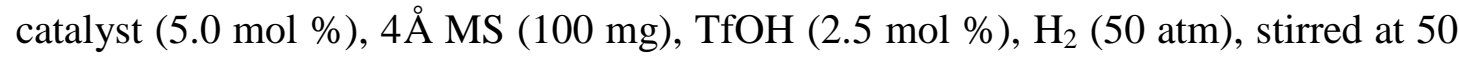
${ }^{\circ} \mathrm{C}$ for $22 \mathrm{~h}$. ${ }^{b}$ The yield were determined by ${ }^{1} \mathrm{H}$ NMR spectroscopy of the crude reaction mixture. ${ }^{c}$ The enantiomeric excesses were determined by HPLC with a chiral IA column. 


\section{General procedure for the synthesis of dibenzo $[c, e]$ azepines $^{2}$}

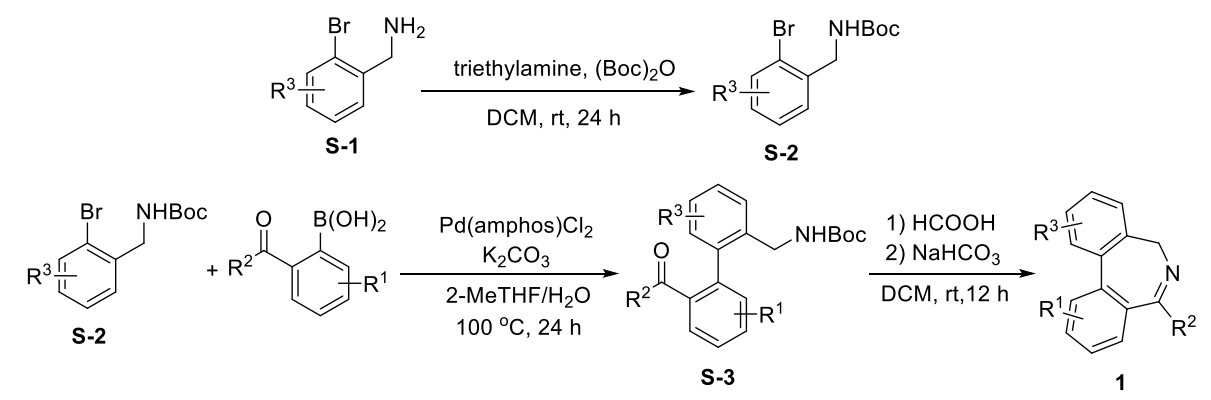

\section{Typical procedure:}

Step 1: To a solution of $\mathbf{S - 1}(5 \mathrm{mmol})$ and triethylamine $(5 \mathrm{mmol})$ in $\mathrm{CH}_{2} \mathrm{Cl}_{2}(20$ $\mathrm{mL}$ ) was added a solution of di-tert-butyl dicarbonate $(7 \mathrm{mmol})$ in $\mathrm{CH}_{2} \mathrm{Cl}_{2}(5 \mathrm{~mL})$. The reaction was stirred at room temperature for $24 \mathrm{~h}$. Water $(20 \mathrm{~mL})$ and $\mathrm{CH}_{2} \mathrm{Cl}_{2}(20$ $\mathrm{mL})$ were added and the organic layer was separated. The aqueous layer was extracted with $\mathrm{CH}_{2} \mathrm{Cl}_{2}(3 \times 20 \mathrm{~mL})$. Then, the combined organic layers were dried over $\mathrm{MgSO}_{4}$ and the solvent was removed under reduced pressure. The crude product was purified by column chromatography on silica gel to afford the $\mathbf{S - 2}$ as a colourless oil.

Step 2: To a solution of $\mathbf{S - 2}(2 \mathrm{mmol})$ and arylboronic acid (1.2 equiv) in 2-methyltetrahydrofuran $(15 \mathrm{~mL})$ and water $(15 \mathrm{~mL})$ was added $\mathrm{K}_{2} \mathrm{CO}_{3}$ (3 equiv). The catalyst $\mathrm{Pd}($ amphos $) \mathrm{Cl}_{2}$ (0.05 equiv) was added under nitrogen atmosphere and the reaction was allowed to stir at $100{ }^{\circ} \mathrm{C}$ for $24 \mathrm{~h}$. The reaction mixture was cooled to room temperature and the organic layer was separated. The aqueous layer was extracted with ethyl acetate $(2 \times 10 \mathrm{~mL})$. The combined organic layers were washed with brine and dried over $\mathrm{MgSO}_{4}$. The solvent was removed under reduced pressure, and the crude product was subjected to column chromatography on silica gel to afford the $\mathbf{S - 3}$ as a colourless oil (eluent: petroleum ether/ethyl acetate $=20 / 1$ to 5/1).

Step 3 : To a solution of $\mathbf{S - 2}$ in $\mathrm{CH}_{2} \mathrm{Cl}_{2}(2 \mathrm{~mL} / \mathrm{g}$ of $\mathbf{S}-2)$ was added $\mathrm{HCOOH}$ (7 $\mathrm{mL} / \mathrm{g}$ of $\mathbf{S - 2}$ ) and the mixture was stirred at room temperature for $12 \mathrm{~h}$. The solvent was firstly removed under reduced pressure. Then the residue was redissolved in $\mathrm{CH}_{2} \mathrm{Cl}_{2}$, and $\mathrm{NaHCO}_{3}$ (2 equiv.) was added and stirred at room temperature for $12 \mathrm{~h}$. 
The precipitate was removed by filtration, then the solvent was removed under reduced pressure and the crude product was subjected to column chromatography on silica gel to afford compound 1 (eluent: petroleum ether/ethyl acetate =10/1 to 5/1).

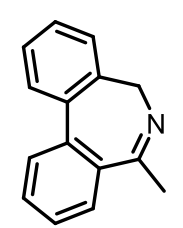

$1 \mathrm{a}$

1a: (Known compound, see: France, S. P.; Aleku, G. A.; Sharma, M.; Mangas-Sanchez, J.; Howard, R. M.; Steflik, J.; Kumar, R.; Adams, R. W.; Slabu, I.; Crook, R.; Grogan, G.; Wallace, T. W.; Turner, N. J. Angew. Chem., Int. Ed. 2017, 56, 15589); white solid, m.p. 108-109 ${ }^{\circ} \mathrm{C}$, isolated yield 90\%; ${ }^{1} \mathrm{H}$ NMR (500 MHz, $\left.\mathrm{CDCl}_{3}\right): \delta(\mathrm{ppm})$ 7.69-7.61 (m, 3H), 7.55-7.52 (m, 1H), 7.47-7.36 (m, 4H), $4.64(\mathrm{~d}, J=10.5 \mathrm{~Hz}, 1 \mathrm{H}), 3.79$ (d, J = 11.0 Hz, 1H), 2.38 (s, $3 \mathrm{H}) ;{ }^{13} \mathrm{C} \mathrm{NMR}\left(125 \mathrm{MHz}, \mathrm{CDCl}_{3}\right): \delta$ (ppm) 167.4, 140.8, 139.2, 138.2, 135.8, 129.6, $129.2,128.4,128.2,127.8,127.7,127.5,127.2,54.9,26.6$.

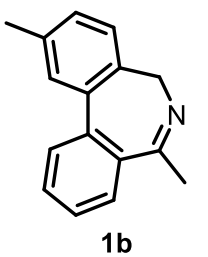

1b: (New compound); white crystal, m.p. $117-118{ }^{\circ} \mathrm{C}$, isolated yield $70 \% ;{ }^{1} \mathrm{H}$ NMR $\left(400 \mathrm{MHz}, \mathrm{CDCl}_{3}\right): 7.68(\mathrm{~d}, J=7.6 \mathrm{~Hz}, 1 \mathrm{H}), 7.64(\mathrm{~d}, J=$ $7.6 \mathrm{~Hz}, 1 \mathrm{H}), 7.54-7.50(\mathrm{~m}, 1 \mathrm{H}), 7.46-7.43(\mathrm{~m}, 2 \mathrm{H}), 7.33(\mathrm{~d}, J=7.6 \mathrm{~Hz}$, 1H), $7.19(\mathrm{~d}, J=7.6 \mathrm{~Hz}, 1 \mathrm{H}), 4.61(\mathrm{~d}, J=10.8 \mathrm{~Hz}, 1 \mathrm{H}), 3.75(\mathrm{~d}, J=$ $10.8 \mathrm{~Hz}, 1 \mathrm{H}), 2.42$ (s, 3H), 2.37 (s, 3H); ${ }^{13} \mathrm{C} \mathrm{NMR}\left(100 \mathrm{MHz}, \mathrm{CDCl}_{3}\right): 167.2,139.3$, 138.2, 138.1, 137.4, 135.9, 129.5, 129.2, 129.1, 128.9, 127.7, 127.4, 127.1, 54.6, 26.6, 21.4. HRMS-ESI exact mass calcd. for $\mathrm{C}_{16} \mathrm{H}_{16} \mathrm{~N}^{+}\left([\mathrm{M}+\mathrm{H}]^{+}\right)$requires $\mathrm{m} / \mathrm{z} 222.1277$, found $\mathrm{m} / \mathrm{z} 222.1274$.

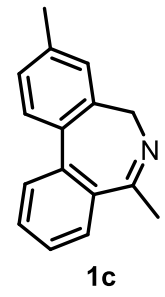

1c: (New compound); white crystal, m.p. $120-121{ }^{\circ} \mathrm{C}$, isolated yield $47 \%$; ${ }^{1} \mathrm{H}$ NMR (400 MHz, $\left.\mathrm{CDCl}_{3}\right)$ : 7.67-7.62 (m, 2H), 7.53-7.50 (m, 2H), 7.44-7.41 (m, 1H), $7.21(\mathrm{~d}, J=7.6 \mathrm{~Hz}, 1 \mathrm{H}), 4.59(\mathrm{~d}, J=10.8 \mathrm{~Hz}, 1 \mathrm{H})$, $3.77(\mathrm{~d}, J=10.8 \mathrm{~Hz}, 1 \mathrm{H}), 2.41(\mathrm{~s}, 3 \mathrm{H}), 2.37$ (s, 3H); ${ }^{13} \mathrm{C}$ NMR $(100 \mathrm{MHz}$, $\left.\mathrm{CDCl}_{3}\right): 167.4,140.7,139.2,138.2,135.7,135.4,129.5,129.0,128.5,128.3,128.2$, 127.7, 126.9, 55.0, 26.6, 21.2. HRMS-ESI exact mass calcd. for $\mathrm{C}_{16} \mathrm{H}_{16} \mathrm{~N}^{+}\left([\mathrm{M}+\mathrm{H}]^{+}\right)$ requires $\mathrm{m} / \mathrm{z} 222.1277$, found $\mathrm{m} / \mathrm{z} 222.1274$. 


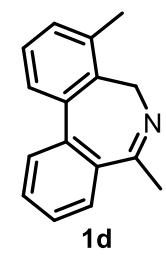

1d: (New compound); white crystal, m.p. $138-139{ }^{\circ} \mathrm{C}$, isolated yield $45 \%$; ${ }^{1} \mathrm{H}$ NMR (400 MHz, $\left.\mathrm{CDCl}_{3}\right): 7.69-7.63(\mathrm{~m}, 2 \mathrm{H}), 7.53-7.49(\mathrm{~m}, 1 \mathrm{H})$, 7.46-7.42 (m, 2H), 7.27-7.23 (m, 2H), 4.95 (d, $J=10.8 \mathrm{~Hz}, 1 \mathrm{H}), 3.49$ (d, $J$ $=10.8 \mathrm{~Hz}, 1 \mathrm{H}), 2.61(\mathrm{~s}, 3 \mathrm{H}), 2.37(\mathrm{~s}, 3 \mathrm{H}) ;{ }^{13} \mathrm{C} \mathrm{NMR}\left(100 \mathrm{MHz}, \mathrm{CDCl}_{3}\right)$ : $167.6,139.9,139.5,138.8,135.7,134.5,130.0,129.4,129.3,127.4,127.1,127.0$, 126.6, 50.3, 26.2, 20.0. HRMS-ESI exact mass calcd. for $\mathrm{C}_{16} \mathrm{H}_{16} \mathrm{~N}^{+}\left([\mathrm{M}+\mathrm{H}]^{+}\right)$requires $\mathrm{m} / \mathrm{z} 222.1277$, found $\mathrm{m} / \mathrm{z} 222.1273$.

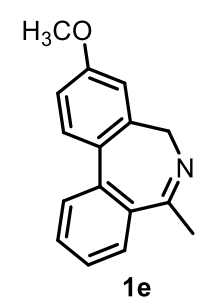

1e: (New compound); yellow solid, m.p. $146-147{ }^{\circ} \mathrm{C}$, isolated yield 62\%; ${ }^{1} \mathrm{H}$ NMR $\left(400 \mathrm{MHz}, \mathrm{CDCl}_{3}\right): 7.63(\mathrm{~d}, J=7.6 \mathrm{~Hz}, 2 \mathrm{H}), 7.54-7.48(\mathrm{~m}, 2 \mathrm{H})$, 7.42-7.39 (m, 1H), 6.99-6.93 (m, 2H), 4.59 (d, $J=10.8 \mathrm{~Hz}, 1 \mathrm{H}), 3.86$ (s, $3 \mathrm{H}), 3.79(\mathrm{~d}, J=10.8 \mathrm{~Hz}, 1 \mathrm{H}), 2.38(\mathrm{~s}, 3 \mathrm{H}) ;{ }^{13} \mathrm{C} \mathrm{NMR}(100 \mathrm{MHz}$, $\left.\mathrm{CDCl}_{3}\right): 168.0,159.9,142.1,139.1,135.5,131.0,129.6,128.9,127.8,126.6,113.9$, 112.5, 55.5, 55.2, 26.7. HRMS-ESI exact mass calcd. for $\mathrm{C}_{16} \mathrm{H}_{16} \mathrm{NO}^{+}\left([\mathrm{M}+\mathrm{H}]^{+}\right)$ requires $\mathrm{m} / \mathrm{z} 238.1226$, found $\mathrm{m} / \mathrm{z} 238.1225$.

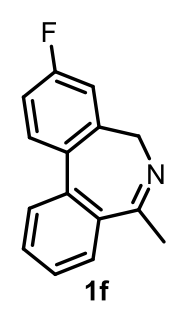

1f: (New compound); white crystal, m.p. $96-97{ }^{\circ} \mathrm{C}$, isolated yield $82 \% ;{ }^{1} \mathrm{H}$ NMR (400 MHz, $\left.\mathrm{CDCl}_{3}\right)$ : 7.65-7.61 (m, 2H), 7.58-7.50 (m, 2H), 7.47-7.43 (m, 1H), 7.17-7.14 (m, 1H), 7.11-7.06 (m, 1H), $4.58(\mathrm{~d}, J=10.8 \mathrm{~Hz}, 1 \mathrm{H})$, $3.77(\mathrm{~d}, J=10.8 \mathrm{~Hz}, 1 \mathrm{H}), 2.37(\mathrm{~s}, 3 \mathrm{H}) ;{ }^{13} \mathrm{C} \mathrm{NMR}\left(100 \mathrm{MHz}, \mathrm{CDCl}_{3}\right)$ : 167.9, $162.9\left(\mathrm{~d}, J_{\mathrm{C}-\mathrm{F}}=247.0 \mathrm{~Hz}\right), 142.7\left(\mathrm{~d}, J_{\mathrm{C}-\mathrm{F}}=8.0 \mathrm{~Hz}\right), 138.3,135.7$, $134.4\left(\mathrm{~d}, J_{\mathrm{C}-\mathrm{F}}=3.0 \mathrm{~Hz}\right), 130.0\left(\mathrm{~d}, J_{\mathrm{C}-\mathrm{F}}=9.0 \mathrm{~Hz}\right), 129.7,129.1,127.8,127.3,114.6(\mathrm{~d}$, $\left.J_{\mathrm{C}-\mathrm{F}}=32.0 \mathrm{~Hz}\right), 114.4\left(\mathrm{~d}, J_{\mathrm{C}-\mathrm{F}}=31.0 \mathrm{~Hz}\right), 54.8,26.6$. HRMS-ESI exact mass calcd. for $\mathrm{C}_{15} \mathrm{H}_{13} \mathrm{NF}^{+}\left([\mathrm{M}+\mathrm{H}]^{+}\right)$requires $\mathrm{m} / \mathrm{z} 226.1027$, found $\mathrm{m} / \mathrm{z} 226.1026$.

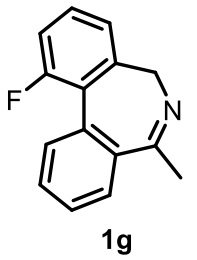

1g: (New compound); white solid, m.p. $108-109{ }^{\circ} \mathrm{C}$, isolated yield 50\%; ${ }^{1} \mathrm{H}$ NMR (500 MHz, $\left.\mathrm{CDCl}_{3}\right)$ : 7.68-7.65 (m, 2H), 7.55-7.47 (m, 2H), 7.39-7.37 (m, 1H), 7.34-7.29 (m, 1H), 7.14-7.10 (m, 1H), $5.12(\mathrm{~d}, J=$ 
$11.0 \mathrm{~Hz}, 1 \mathrm{H}), 3.46(\mathrm{~d}, J=11.0 \mathrm{~Hz}, 1 \mathrm{H}), 2.38(\mathrm{~s}, 3 \mathrm{H}) ;{ }^{13} \mathrm{C} \mathrm{NMR}\left(125 \mathrm{MHz}, \mathrm{CDCl}_{3}\right)$ : $168.5,159.0\left(\mathrm{~d}, J_{\mathrm{C}-\mathrm{F}}=245.0 \mathrm{~Hz}\right), 140.7\left(\mathrm{~d}, J_{\mathrm{C}-\mathrm{F}}=5.0 \mathrm{~Hz}\right), 138.2\left(\mathrm{~d}, J_{\mathrm{C}-\mathrm{F}}=2.5 \mathrm{~Hz}\right)$, $135.8,129.7,129.3,128.3\left(\mathrm{~d}, J_{\mathrm{C}-\mathrm{F}}=8.8 \mathrm{~Hz}\right), 128.1,127.9,127.8\left(\mathrm{~d}, J_{\mathrm{C}-\mathrm{F}}=3.8 \mathrm{~Hz}\right)$, $123.9\left(\mathrm{~d}, J_{\mathrm{C}-\mathrm{F}}=3.8 \mathrm{~Hz}\right), 114.7\left(\mathrm{~d}, J_{\mathrm{C}-\mathrm{F}}=22.5 \mathrm{~Hz}\right), 45.7\left(\mathrm{~d}, J_{\mathrm{C}-\mathrm{F}}=5.0 \mathrm{~Hz}\right), 26.6$. HRMS-ESI exact mass calcd. for $\mathrm{C}_{15} \mathrm{H}_{13} \mathrm{NF}^{+}\left([\mathrm{M}+\mathrm{H}]^{+}\right)$requires $\mathrm{m} / \mathrm{z} 226.1027$, found $\mathrm{m} / \mathrm{z} 226.1023$

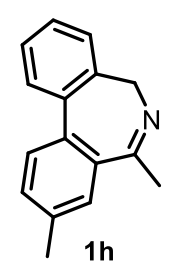

1h: (New compound); yellow oil, isolated yield 75\%; ${ }^{1} \mathrm{H}$ NMR $(400 \mathrm{MHz}$, $\left.\mathrm{CDCl}_{3}\right)$ : 7.61-7.57 (m, 2H), 7.45-7.34 (m, 5H), $4.63(\mathrm{~d}, J=10.8 \mathrm{~Hz}, 1 \mathrm{H})$, $3.78(\mathrm{~d}, J=10.8 \mathrm{~Hz}, 1 \mathrm{H}), 2.48(\mathrm{~s}, 3 \mathrm{H}), 2.37(\mathrm{~s}, 3 \mathrm{H}) ;{ }^{13} \mathrm{C} \mathrm{NMR}(100 \mathrm{MHz}$, $\left.\mathrm{CDCl}_{3}\right): 167.4,140.5,138.2,137.1,136.5,135.7,130.5,129.1,128.2$, 128.0, 127.9, 127.7, 127.5, 55.0, 26.6, 21.4. HRMS-ESI exact mass calcd. for $\mathrm{C}_{16} \mathrm{H}_{16} \mathrm{~N}^{+}\left([\mathrm{M}+\mathrm{H}]^{+}\right)$requires $\mathrm{m} / \mathrm{z} 222.1277$, found $\mathrm{m} / \mathrm{z} 222.1275$.

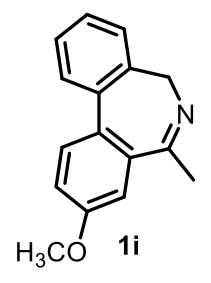

1i: (New compound); yellow oil, isolated yield 55\%; ${ }^{1} \mathrm{H}$ NMR (400 $\left.\mathrm{MHz}, \mathrm{CDCl}_{3}\right)$ : 7.62-7.55 (m, 2H), 7.43-7.31 (m, 3H), 7.13-7.08 (m, 2H), $4.64(\mathrm{~d}, J=10.4 \mathrm{~Hz}, 1 \mathrm{H}), 3.90(\mathrm{~s}, 3 \mathrm{H}), 3.78(\mathrm{~d}, J=10.4 \mathrm{~Hz}, 1 \mathrm{H}), 2.36$ $(\mathrm{s}, 3 \mathrm{H}) ;{ }^{13} \mathrm{C} \mathrm{NMR}\left(100 \mathrm{MHz}, \mathrm{CDCl}_{3}\right): 166.9,158.4,140.1,138.0,136.9$, 132.0, 130.6, 128.0, 127.7, 127.5, 127.5, 115.7, 112.4, 55.6, 55.1, 26.6. HRMS-ESI exact mass calcd. for $\mathrm{C}_{16} \mathrm{H}_{16} \mathrm{NO}^{+}\left([\mathrm{M}+\mathrm{H}]^{+}\right)$requires $\mathrm{m} / \mathrm{z}$ 238.1226, found $\mathrm{m} / \mathrm{z}$ 238.1227 .

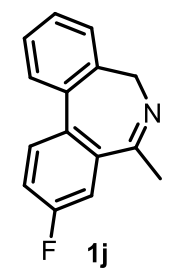

1j: (New compound); yellow solid, m.p. $74-75{ }^{\circ} \mathrm{C}$, isolated yield $45 \% ;{ }^{1} \mathrm{H}$ NMR (400 MHz, $\left.\mathrm{CDCl}_{3}\right)$ : 7.68-7.65 (m, 1H), 7.57-7.56 (m, 1H), 7.45-7.31 $(\mathrm{m}, 4 \mathrm{H}), 7.26-7.22(\mathrm{~m}, 1 \mathrm{H}), 4.67\left(\mathrm{dd}, J_{1}=10.8 \mathrm{~Hz}, J_{2}=1.6 \mathrm{~Hz}, 1 \mathrm{H}\right), 3.76$ $(\mathrm{d}, J=11.0 \mathrm{~Hz}, 1 \mathrm{H}), 2.35$ (s, 3H); $\left.{ }^{13} \mathrm{C} \mathrm{NMR} \mathrm{(100} \mathrm{MHz,} \mathrm{CDCl}_{3}\right): 166.2$, $161.4\left(\mathrm{~d}, J_{\mathrm{C}-\mathrm{F}}=247.0 \mathrm{~Hz}\right), 140.4,137.4,137.3\left(\mathrm{~d}, J_{\mathrm{C}-\mathrm{F}}=7.0 \mathrm{~Hz}\right), 135.5,131.3\left(\mathrm{~d}, J_{\mathrm{C}-\mathrm{F}}\right.$ $=8.0 \mathrm{~Hz}), 128.4,128.3,128.0,127.7,117.1\left(\mathrm{~d}, J_{\mathrm{C}-\mathrm{F}}=22.0 \mathrm{~Hz}\right), 114.1\left(\mathrm{~d}, J_{\mathrm{C}-\mathrm{F}}=21.0\right.$ $\mathrm{Hz})$, 55.0, 26.4. HRMS-ESI exact mass calcd. for $\mathrm{C}_{15} \mathrm{H}_{13} \mathrm{NF}^{+}\left([\mathrm{M}+\mathrm{H}]^{+}\right)$requires $\mathrm{m} / \mathrm{z}$ 226.1027, found $\mathrm{m} / \mathrm{z} 226.1025$. 


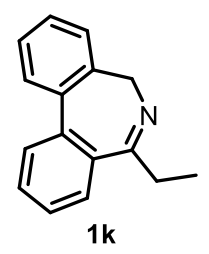

1k: (New compound); yellow oil, isolated yield 55\%; ${ }^{1} \mathrm{H}$ NMR (400 $\left.\mathrm{MHz}, \mathrm{CDCl}_{3}\right)$ : 7.69-7.61 (m, 3H), 7.55-7.51 (m, 1H), 7.47-7.35 (m, 4H), $4.67(\mathrm{~d}, J=10.4 \mathrm{~Hz}, 1 \mathrm{H}), 3.79$ (d, $J=10.8 \mathrm{~Hz}, 1 \mathrm{H}), 2.83-2.74(\mathrm{~m}, 1 \mathrm{H})$, 2.70-2.60 (m, 1H), $0.98(\mathrm{t}, J=7.4 \mathrm{~Hz}, 3 \mathrm{H}) ;{ }^{13} \mathrm{C} \mathrm{NMR}(100 \mathrm{MHz}$, $\left.\mathrm{CDCl}_{3}\right): 171.5,141.2,139.7,138.3,135.5,129.4,129.3,128.3,128.0,127.7,127.6$, 127.4, 127.2, 54.8, 32.3, 11.5. HRMS-ESI exact mass calcd. for $\mathrm{C}_{16} \mathrm{H}_{16} \mathrm{~N}^{+}\left([\mathrm{M}+\mathrm{H}]^{+}\right)$ requires $\mathrm{m} / \mathrm{z} 222.1277$, found $\mathrm{m} / \mathrm{z} 222.1279$.

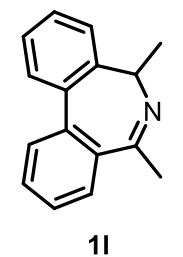

11: (Known compound, see: France, S. P.; Aleku, G. A.; Sharma, M.; Mangas-Sanchez, J.; Howard, R. M.; Steflik, J.; Kumar, R.; Adams, R. W.; Slabu, I.; Crook, R.; Grogan, G.; Wallace, T. W.; Turner, N. J. Angew. Chem., Int. Ed. 2017, 56, 15589); yellow solid, m.p. $84-85{ }^{\circ} \mathrm{C}$, isolated yield 45\%; ${ }^{1} \mathrm{H}$ NMR (500 MHz, $\left.\mathrm{CDCl}_{3}\right)$ : 7.70-7.65 (m, 2H), $7.60(\mathrm{~d}, J=7.5 \mathrm{~Hz}, 1 \mathrm{H})$, 7.55-7.52 (m, 2H), 7.48-7.42 (m, 2H), 7.39-7.36 (m, 1H), $3.80(\mathrm{q}, J=6.5 \mathrm{~Hz}, 1 \mathrm{H})$, $2.36(\mathrm{~s}, 3 \mathrm{H}), 1.79(\mathrm{~d}, J=6.5 \mathrm{~Hz}, 3 \mathrm{H}) ;{ }^{13} \mathrm{C} \mathrm{NMR}\left(125 \mathrm{MHz}, \mathrm{CDCl}_{3}\right): 165.1,144.0$, $139.2,137.9,136.1,129.5,129.2,128.6,128.1,127.2,127.2,127.2,123.1,55.9,26.4$, 19.1.

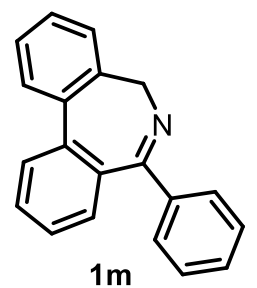

1m: (New compound); white solid, m.p. $56-58{ }^{\circ} \mathrm{C}$, isolated yield 63\%; ${ }^{1} \mathrm{H}$ NMR (400 MHz, $\left.\mathrm{CDCl}_{3}\right): 7.76(\mathrm{~d}, J=8.0 \mathrm{~Hz}, 1 \mathrm{H})$, 7.71-7.69 (m, 1H), 7.62-7.56 (m, 1H), 7.52-7.50 (m, 3H), 7.44-7.29 $(\mathrm{m}, 7 \mathrm{H}), 4.92(\mathrm{~d}, J=10.4 \mathrm{~Hz}, 1 \mathrm{H}), 3.96(\mathrm{~d}, J=10.4 \mathrm{~Hz}, 1 \mathrm{H}) ;{ }^{13} \mathrm{C}$ NMR (100 MHz, $\left.\mathrm{CDCl}_{3}\right)$ : 168.6, 141.1, 141.1, 140.8, 138.3, 134.3, 130.6, 129.8, 129.7, 129.6, 129.0, 128.3, 128.2, 128.1, 127.9, 127.8, 126.4, 55.5. HRMS-ESI exact mass calcd. for $\mathrm{C}_{20} \mathrm{H}_{16} \mathrm{~N}^{+}\left([\mathrm{M}+\mathrm{H}]^{+}\right)$requires $\mathrm{m} / \mathrm{z} 270.1277$, found $\mathrm{m} / \mathrm{z} 270.1276$.

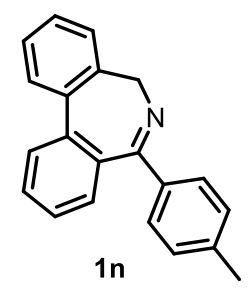

1n: (New compound); white solid, m.p. 121-122 ${ }^{\circ} \mathrm{C}$, isolated yield 73\%; ${ }^{1} \mathrm{H}$ NMR (400 MHz, $\left.\mathrm{CDCl}_{3}\right):$ 7.76-7.68 (m, 2H), 7.59-7.35 (m, $8 \mathrm{H}), 7.12(\mathrm{~d}, J=8.0 \mathrm{~Hz}, 2 \mathrm{H}), 4.89(\mathrm{~d}, J=10.4 \mathrm{~Hz}, 1 \mathrm{H}), 3.94(\mathrm{~d}, J=$ 
$10.4 \mathrm{~Hz}, 1 \mathrm{H}), 2.35$ (s, 3H); ${ }^{13} \mathrm{C}$ NMR (100 MHz, $\left.\mathrm{CDCl}_{3}\right): 168.5,141.2,141.1,139.8$, 138.4, 138.1, 134.4, 130.6, 129.7, 129.6, 129.0, 128.8, 128.3, 128.1, 127.8, 127.8, 126.4, 55.5, 21.4. HRMS-ESI exact mass calcd. for $\mathrm{C}_{21} \mathrm{H}_{18} \mathrm{~N}^{+}\left([\mathrm{M}+\mathrm{H}]^{+}\right)$requires $\mathrm{m} / \mathrm{z}$ 284.1434, found $\mathrm{m} / \mathrm{z} 284.1433$.

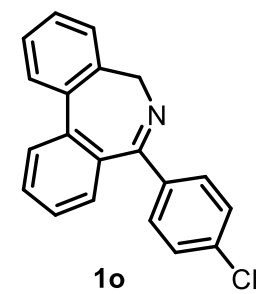

1o: (New compound), white solid, m.p. $119-120{ }^{\circ} \mathrm{C}$, isolated yield 33\%; ${ }^{1} \mathrm{H}$ NMR (400 MHz, $\left.\mathrm{CDCl}_{3}\right): 7.76(\mathrm{~d}, J=7.6 \mathrm{~Hz}, 1 \mathrm{H})$, 7.70-7.68 (m, 1H), 7.63-7.59 (m, 1H), 7.52-7.35 (m, 7H), 7.29 (d, $J=$ $8.4 \mathrm{~Hz}, 2 \mathrm{H}), 4.91(\mathrm{~d}, J=10.4 \mathrm{~Hz}, 1 \mathrm{H}), 3.94(\mathrm{~d}, J=10.8 \mathrm{~Hz}, 1 \mathrm{H}) ;{ }^{13} \mathrm{C}$ NMR (100 MHz, $\left.\mathrm{CDCl}_{3}\right)$ : 167.6, 141.2, 140.9, 139.1, 138.2, 136.0, 133.7, 131.0, 130.4, 130.1, 129.2, 128.4, 128.3, 128.0, 127.9, 126.6, 55.5. HRMS-ESI exact mass calcd. for $\mathrm{C}_{20} \mathrm{H}_{15} \mathrm{NCl}^{+}\left([\mathrm{M}+\mathrm{H}]^{+}\right)$requires $\mathrm{m} / \mathrm{z}$ 304.0888, found $\mathrm{m} / \mathrm{z} 304.0888$.

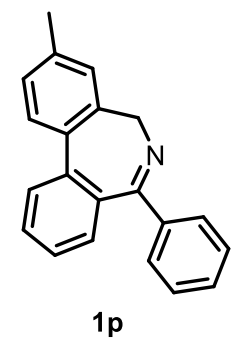

1p: (New compound); white solid, m.p. $108-109{ }^{\circ} \mathrm{C}$, isolated yield 75\%; ${ }^{1} \mathrm{H}$ NMR (400 MHz, $\left.\mathrm{CDCl}_{3}\right): \delta(\mathrm{ppm}) 7.72(\mathrm{~d}, J=7.6 \mathrm{~Hz}, 1 \mathrm{H})$, 7.58-7.53 (m, 2H), 7.50-7.48 (m, 2H), 7.38-7.27 (m, 6H), 7.24-7.20 (m, 1H), $4.85(\mathrm{~d}, J=10.4 \mathrm{~Hz}, 1 \mathrm{H}), 3.93(\mathrm{~d}, J=10.4 \mathrm{~Hz}, 1 \mathrm{H}), 2.38(\mathrm{~s}, 3 \mathrm{H})$; ${ }^{13} \mathrm{C}$ NMR $\left(100 \mathrm{MHz}, \mathrm{CDCl}_{3}\right) \delta(\mathrm{ppm})$ 168.7, 141.1, 140.9, 138.2, 135.6, 134.2, 130.6, 129.8, 129.7, 129.6, 128.8, 128.6, 128.5, 128.2, 128.0, 126.1, 55.6, 21.2. HRMS-ESI exact mass calcd. for $\mathrm{C}_{21} \mathrm{H}_{18} \mathrm{~N}^{+}\left([\mathrm{M}+\mathrm{H}]^{+}\right)$requires $\mathrm{m} / \mathrm{z}$ 284.1434, found $\mathrm{m} / \mathrm{z} 284.1433$.

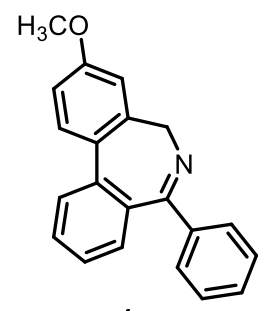

1q: (New compound); white solid, m.p. $49-50{ }^{\circ} \mathrm{C}$, isolated yield 90\%; ${ }^{1} \mathrm{H}$ NMR (400 MHz, $\left.\mathrm{CDCl}_{3}\right): 7.71(\mathrm{~d}, J=8.0 \mathrm{~Hz}, 1 \mathrm{H}), 7.63-7.51(\mathrm{~m}$, 4H), 7.39-7.30 (m, 5H), 7.07 (s, 1H), 6.98-6.96 (m, 1H), 4.88 (d, $J=$ $10.4 \mathrm{~Hz}, 1 \mathrm{H}), 3.97(\mathrm{~d}, J=10.4 \mathrm{~Hz}, 1 \mathrm{H}), 3.84$ (s, 3H); ${ }^{13} \mathrm{C}$ NMR (100 $\left.\mathrm{MHz}, \mathrm{CDCl}_{3}\right): 168.9,159.8,142.2,140.9,133.9,131.0,130.6,129.7$, 129.7, 129.6, 129.4, 128.6, 128.0, 125.7, 114.0, 112.7, 55.8, 55.4. HRMS-ESI exact mass calcd. for $\mathrm{C}_{21} \mathrm{H}_{18} \mathrm{NO}^{+}\left([\mathrm{M}+\mathrm{H}]^{+}\right)$requires $\mathrm{m} / \mathrm{z} 300.1383$, found $\mathrm{m} / \mathrm{z} 300.1381$. 


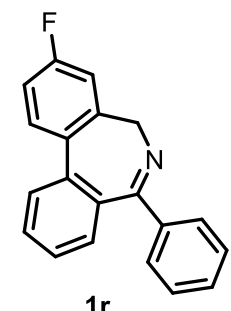

1r: (New compound); white solid, m.p. 126-127 ${ }^{\circ} \mathrm{C}$, isolated yield 35\%; ${ }^{1} \mathrm{H}$ NMR (400 MHz, $\left.\mathrm{CDCl}_{3}\right)$ : 7.71-7.31 (m, 10H), 7.24-7.21 (m, 1H), 7.13-7.08 (m, 1H), $4.86(\mathrm{~d}, J=10.8 \mathrm{~Hz}, 1 \mathrm{H}), 3.94(\mathrm{~d}, J=10.4 \mathrm{~Hz}, 1 \mathrm{H})$

${ }^{13} \mathrm{C}$ NMR (100 MHz, $\left.\mathrm{CDCl}_{3}\right): 169.1,162.9\left(\mathrm{~d}, J_{\mathrm{C}-\mathrm{F}}=247.0 \mathrm{~Hz}\right), 142.9$ $\left(\mathrm{d}, J_{\mathrm{C}-\mathrm{F}}=7.0 \mathrm{~Hz}\right), 140.6,140.1,134.4\left(\mathrm{~d}, J_{\mathrm{C}-\mathrm{F}}=3.0 \mathrm{~Hz}\right), 134.0,130.7$, $129.9\left(\mathrm{~d}, J_{\mathrm{C}-\mathrm{F}}=6.0 \mathrm{~Hz}\right), 129.8,129.6,128.8,128.1,126.5,114.8\left(\mathrm{~d}, J_{\mathrm{C}-\mathrm{F}}=22.0 \mathrm{~Hz}\right)$, $114.6\left(\mathrm{~d}, J_{\mathrm{C}-\mathrm{F}}=22.0 \mathrm{~Hz}\right)$, 55.3. HRMS-ESI exact mass calcd. for $\mathrm{C}_{20} \mathrm{H}_{15} \mathrm{NF}^{+}\left([\mathrm{M}+\mathrm{H}]^{+}\right)$ requires $\mathrm{m} / \mathrm{z} 288.1183$, found $\mathrm{m} / \mathrm{z} 288.1178$.

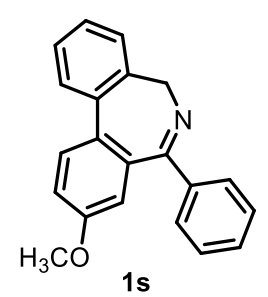

1s: (New compound); colorless oil, isolated yield 70\%; ${ }^{1} \mathrm{H}$ NMR $\left(500 \mathrm{MHz}, \mathrm{CDCl}_{3}\right): \delta(\mathrm{ppm}) 7.69(\mathrm{~d}, J=8.5 \mathrm{~Hz}, 1 \mathrm{H}), 7.65(\mathrm{~d}, J=7.5$ $\mathrm{Hz}, 1 \mathrm{H}), 7.54$ (d, $J=7.0 \mathrm{~Hz}, 2 \mathrm{H}), 7.49$ (d, $J=7.5 \mathrm{~Hz}, 1 \mathrm{H}), 7.41-7.30$ $(\mathrm{m}, 5 \mathrm{H}), 7.16\left(\mathrm{dd}, J_{1}=13.8 \mathrm{~Hz}, J_{2}=2.8 \mathrm{~Hz}, 1 \mathrm{H}\right), 6.89(\mathrm{~d}, J=2.5 \mathrm{~Hz}$, $1 \mathrm{H}), 4.91(\mathrm{~d}, J=10.5 \mathrm{~Hz}, 1 \mathrm{H}), 3.96(\mathrm{~d}, J=10.0 \mathrm{~Hz}, 1 \mathrm{H}), 3.78(\mathrm{~s}, 3 \mathrm{H}) ;{ }^{13} \mathrm{C}$ NMR $(125$ $\left.\mathrm{MHz}, \mathrm{CDCl}_{3}\right) \delta(\mathrm{ppm}) 168.2,157.7,140.7,140.4,138.2,135.3,134.1,130.4,129.8$, 129.6, 128.3, 128.1, 128.0, 127.9, 127.8, 127.6, 116.6, 114.7, 55.7, 55.6. HRMS-ESI exact mass calcd. for $\mathrm{C}_{21} \mathrm{H}_{18} \mathrm{NO}^{+}\left([\mathrm{M}+\mathrm{H}]^{+}\right)$requires $\mathrm{m} / \mathrm{z}$ 300.1383, found $\mathrm{m} / \mathrm{z}$ 300.1383 .

\section{General procedure for the asymmetric hydrogenation of dibenzo[c,e]azepines}
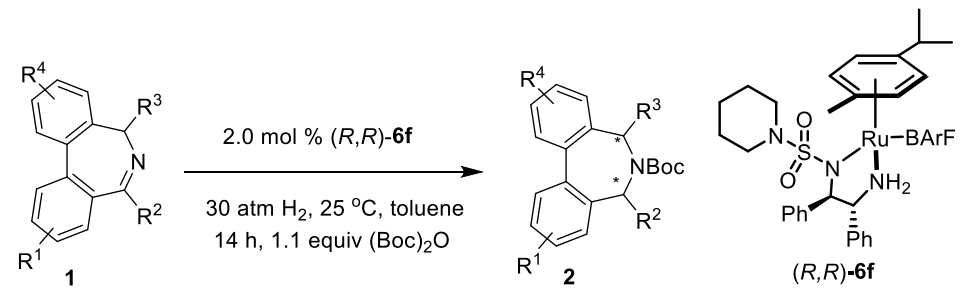

Typical procedure: A $30 \mathrm{~mL}$ glass-lined stainless-steel reactor equipped with a magnetic stirrer bar was charged with $\mathrm{Ru}$-catalyst $(R, R)-\mathbf{6 f}(3 \mathrm{mg}, 2.0 \mathrm{~mol} \%)$, corresponding imine $1(0.1 \mathrm{mmol})$ and $(\mathrm{Boc})_{2} \mathrm{O}(24 \mathrm{mg} .0 .11 \mathrm{mmol})$ in toluene $(1 \mathrm{~mL})$ 
under nitrogen atmosphere in a glove box. The autoclave was closed, and the final pressure of the hydrogen gas was adjusted to 30 atm after purging the autoclave with hydrogen gas several times. The reaction mixture was stirred at $25^{\circ} \mathrm{C}$ for $14 \mathrm{~h}$. Then the hydrogen gas was carefully released and the conversion was determined by ${ }^{1} \mathrm{H}$ NMR. The reaction mixture was filtered through a short pad of silica (petroleum/ethyl acetate, $10 / 1, v / v)$ to give the pure product. The enatiomeric excess of the product was determined by HPLC with a chiral column.

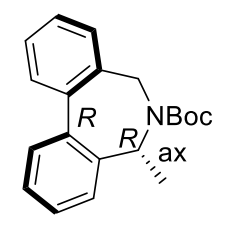

$(\mathrm{a} R, 5 R)-\mathbf{2 a}$

(aR,5R)-2a: (Known compound, see: Cheetham, C. A.; Massey, R. S.; Pira, S. L.; Pritchard, R. G.; Wallace, T. W. Org. Biomol. Chem. 2011, 9, 1831); colorless oil, $30.3 \mathrm{mg}, 98 \%$ yield, $94 \%$ ee, $[\alpha]_{\mathrm{D}}{ }^{25}=+313.6(c$ $\left.0.5, \mathrm{CHCl}_{3}\right),\left[\right.$ Lit. $^{3}[\alpha]_{\mathrm{D}}^{24}=-322\left(\mathrm{c} 0.61, \mathrm{CHCl}_{3}\right), 99 \%$ ee for $(S)$ enantiomer]; ${ }^{1} \mathrm{H}$ NMR (400 MHz, $\left.\mathrm{CDCl}_{3}\right): \delta(\mathrm{ppm})$ 7.53-7.35 (m, 8H), 5.14-5.04 (m, br, $2 \mathrm{H}), 3.73(\mathrm{~d}, J=12.8 \mathrm{~Hz}, 1 \mathrm{H}), 1.54(\mathrm{~s}, 9 \mathrm{H}), 0.87(\mathrm{~d}, J=6.8 \mathrm{~Hz}, 3 \mathrm{H}) ;{ }^{13} \mathrm{C} \mathrm{NMR}$ $\left(100 \mathrm{MHz}, \mathrm{CDCl}_{3}\right) \delta$ (ppm) 153.8, 141.1, 139.3, 135.4, 130.3, 129.7, 129.3, 128.7, 128.4, 128.3, 127.7, 79.8, 57.7, 46.7, 28.7, 21.5. HRMS-ESI exact mass calcd. for $\mathrm{C}_{20} \mathrm{H}_{24} \mathrm{O}_{2} \mathrm{~N}^{+}\left([\mathrm{M}+\mathrm{H}]^{+}\right)$requires $\mathrm{m} / \mathrm{z} 310.18016$, found $\mathrm{m} / \mathrm{z} 310.17969$.

The enantiomeric excess was determined by HPLC on Chiralcel IC column (hexane : isopropanol $=95: 5$, flowing rate $=1.0 \mathrm{~mL} / \mathrm{min}, 25{ }^{\circ} \mathrm{C}$, $\mathrm{UV}$ detection at $\lambda=254 \mathrm{~nm}$ ), $\mathrm{t}_{\mathrm{R} 1}=5.1 \min$ (major), $\mathrm{t}_{\mathrm{R} 2}=6.1 \mathrm{~min}$ (minor).

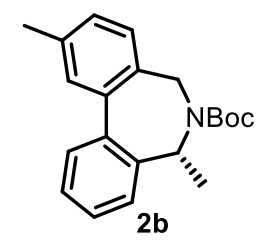

(+)-2b: (New compound); white solid, m.p. 121-122 ${ }^{\circ} \mathrm{C}, 30.0 \mathrm{mg}$, $93 \%$ yield, $93 \%$ ee, $[\alpha]_{\mathrm{D}}{ }^{25}=+290.0\left(c 0.5, \mathrm{CHCl}_{3}\right) ;{ }^{1} \mathrm{H} \mathrm{NMR}(400$ $\left.\mathrm{MHz}, \mathrm{CDCl}_{3}\right): \delta(\mathrm{ppm}) 7.49(\mathrm{~d}, J=7.6 \mathrm{~Hz}, 1 \mathrm{H}), 7.44-7.40(\mathrm{~m}, 1 \mathrm{H})$, 7.36-7.25 (m, 4H), $7.16(\mathrm{~d}, J=7.6 \mathrm{~Hz}, 1 \mathrm{H}), 5.12-4.97(\mathrm{~m}, 2 \mathrm{H}), 3.68$ $(\mathrm{d}, J=13.2 \mathrm{~Hz}, 1 \mathrm{H}), 2.44(\mathrm{~s}, 3 \mathrm{H}), 1.53(\mathrm{~s}, 9 \mathrm{H}), 0.87(\mathrm{~d}, J=6.8 \mathrm{~Hz}, 3 \mathrm{H}) ;{ }^{13} \mathrm{C} \mathrm{NMR}$ $\left(100 \mathrm{MHz}, \mathrm{CDCl}_{3}\right) \delta(\mathrm{ppm}) 153.8,141.0,139.4,139.3,138.3,132.6,130.3,129.7$, 129.2, 128.9, 128.4, 128.3, 128.2, 79.8, 57.8, 46.4, 28.8, 21.5, 21.5. HRMS-ESI exact mass calcd. for $\mathrm{C}_{21} \mathrm{H}_{26} \mathrm{O}_{2} \mathrm{~N}^{+}\left([\mathrm{M}+\mathrm{H}]^{+}\right)$requires $\mathrm{m} / \mathrm{z} 324.1958$, found $\mathrm{m} / \mathrm{z} 324.1952$. 
The enantiomeric excess was determined by HPLC on Chiralcel IC column (hexane : isopropanol $=95: 5$, flowing rate $=1.0 \mathrm{~mL} / \mathrm{min}, 25^{\circ} \mathrm{C}$, $\mathrm{UV}$ detection at $\lambda=254 \mathrm{~nm}$ ), $\mathrm{t}_{\mathrm{R} 1}=5.1 \min$ (major), $\mathrm{t}_{\mathrm{R} 2}=5.9 \min$ (minor).

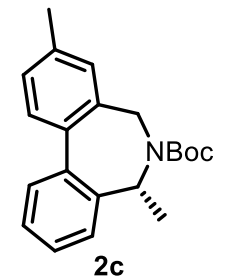

(+)-2c: (New compound); colorless oil, $31.0 \mathrm{mg}, 96 \%$ yield, $96 \%$ ee, $[\alpha]_{\mathrm{D}}{ }^{25}=+354.2\left(c 0.5, \mathrm{CHCl}_{3}\right) ;{ }^{1} \mathrm{H} \mathrm{NMR}\left(400 \mathrm{MHz}, \mathrm{CDCl}_{3}\right): \delta(\mathrm{ppm})$ $7.47(\mathrm{~d}, J=7.6 \mathrm{~Hz}, 1 \mathrm{H}), 7.43-7.39(\mathrm{~m}, 2 \mathrm{H}), 7.33-7.31(\mathrm{~m}, 2 \mathrm{H})$, 7.25-7.24 (m, 2H), 5.11-4.98 (m, 2H), 3.69 (d, $J=12.0 \mathrm{~Hz}, 1 \mathrm{H}), 2.41(\mathrm{~s}$ $3 \mathrm{H}), 1.53(\mathrm{~s}, 9 \mathrm{H}), 0.87(\mathrm{~d}, J=7.2 \mathrm{~Hz}, 3 \mathrm{H}) ;{ }^{13} \mathrm{C} \mathrm{NMR}\left(100 \mathrm{MHz}, \mathrm{CDCl}_{3}\right) \delta(\mathrm{ppm})$ $153.8,139.3,139.2,138.2,135.2,130.3,130.0,129.6,129.3,128.3,128.0,127.6$, 79.8, 57.8, 47.2, 28.8, 21.6, 21.3. HRMS-ESI exact mass calcd. for $\mathrm{C}_{21} \mathrm{H}_{26} \mathrm{O}_{2} \mathrm{~N}^{+}\left([\mathrm{M}+\mathrm{H}]^{+}\right)$requires $\mathrm{m} / \mathrm{z} 324.1958$, found $\mathrm{m} / \mathrm{z} 324.1953$.

The enantiomeric excess was determined by HPLC on Chiralcel IC column (hexane : isopropanol $=95: 5$, flowing rate $=1.0 \mathrm{~mL} / \mathrm{min}, 25{ }^{\circ} \mathrm{C}$, $\mathrm{UV}$ detection at $\lambda=254 \mathrm{~nm}$ ), $\mathrm{t}_{\mathrm{R} 1}=5.0 \mathrm{~min}$ (major), $\mathrm{t}_{\mathrm{R} 2}=6.5 \mathrm{~min}$ (minor).

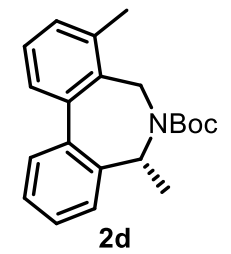

(+)-2d: (New compound); colorless oil, $31.0 \mathrm{mg}, 96 \%$ yield, $92 \%$ ee, $[\alpha]_{\mathrm{D}}{ }^{25}=+278.0\left(c 0.5, \mathrm{CHCl}_{3}\right) ;{ }^{1} \mathrm{H} \mathrm{NMR}\left(400 \mathrm{MHz}, \mathrm{CDCl}_{3}\right): \delta(\mathrm{ppm})$ $7.49(\mathrm{~d}, J=7.6 \mathrm{~Hz}, 1 \mathrm{H}), 7.44-7.40(\mathrm{~m}, 1 \mathrm{H}), 7.34-7.28(\mathrm{~m}, 4 \mathrm{H})$, 7.25-7.22 (m, 1H), 5.38-5.02 (m, 2H), $3.53(\mathrm{~s}, \mathrm{br}, 1 \mathrm{H}), 2.52(\mathrm{~s}, 3 \mathrm{H})$, $1.54(\mathrm{~s}, 9 \mathrm{H}), 0.83(\mathrm{~d}, J=6.4 \mathrm{~Hz}, 3 \mathrm{H}) ;{ }^{13} \mathrm{C} \mathrm{NMR}\left(100 \mathrm{MHz}, \mathrm{CDCl}_{3}\right) \delta(\mathrm{ppm})$ 153.4, $141.7,139.9,139.3,134.0,130.0,129.8,128.3,128.2,128.1,125.8,79.8,58.0,42.7$, 28.7, 27.5, 21.1, 20.0. HRMS-ESI exact mass calcd. for $\mathrm{C}_{21} \mathrm{H}_{26} \mathrm{O}_{2} \mathrm{~N}^{+}\left([\mathrm{M}+\mathrm{H}]^{+}\right)$ requires $\mathrm{m} / \mathrm{z} 324.1958$, found $\mathrm{m} / \mathrm{z} 324.1956$.

The enantiomeric excess was determined by HPLC on Chiralcel IC column (hexane : isopropanol $=99: 1$, flowing rate $=1.0 \mathrm{~mL} / \mathrm{min}, 25{ }^{\circ} \mathrm{C}$, $\mathrm{UV}$ detection at $\lambda=254 \mathrm{~nm}$ ), $\mathrm{t}_{\mathrm{R} 1}=6.2 \min$ (major), $\mathrm{t}_{\mathrm{R} 2}=7.0 \mathrm{~min}$ (minor). 


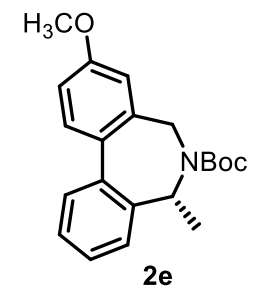

(+)-2e: (New compound); colorless oil, $32.0 \mathrm{mg}$, 94\% yield, 94\% ee, $[\alpha]_{\mathrm{D}}{ }^{25}=+343.2\left(c 1.0, \mathrm{CHCl}_{3}\right) ;{ }^{1} \mathrm{H} \mathrm{NMR}\left(400 \mathrm{MHz}, \mathrm{CDCl}_{3}\right): \delta(\mathrm{ppm})$ 7.45-7.39 (m, 3H), 7.32-7.31 (m, 2H), 6.99-6.97 (m, 2H), 5.09 (s, br, 2H), 3.87 (s, 3H), 3.70 (d, $J=12.0 \mathrm{~Hz}, 1 \mathrm{H}), 1.53(\mathrm{~s}, 9 \mathrm{H}), 0.89$ (d, $J=$ $6.8 \mathrm{~Hz}, 3 \mathrm{H}) ;{ }^{13} \mathrm{C}$ NMR $\left(100 \mathrm{MHz}, \mathrm{CDCl}_{3}\right) \delta(\mathrm{ppm}) 159.7,139.1,139.0,136.7,133.7$, $130.3,129.5,128.9,128.3,127.7,114.6,79.9,57.8,55.6,47.1,28.8,21.5$. HRMS-ESI exact mass calcd. for $\mathrm{C}_{21} \mathrm{H}_{26} \mathrm{NO}_{3}{ }^{+}\left([\mathrm{M}+\mathrm{H}]^{+}\right)$requires $\mathrm{m} / \mathrm{z} 340.1907$, found $\mathrm{m} / \mathrm{z} 340.1910$.

The enantiomeric excess was determined by HPLC on Chiralcel IC column (hexane : isopropanol $=95: 5$, flowing rate $=1.0 \mathrm{~mL} / \mathrm{min}, 25^{\circ} \mathrm{C}$, $\mathrm{UV}$ detection at $\lambda=254 \mathrm{~nm}$ ), $\mathrm{t}_{\mathrm{R} 1}=6.3 \min$ (major), $\mathrm{t}_{\mathrm{R} 2}=8.5 \min$ (minor).

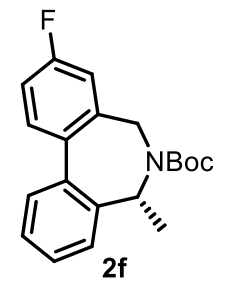

(+)-2f: (New compound); colorless oil, $31.0 \mathrm{mg}$, 95\% yield, $87 \%$ ee, $[\alpha]_{\mathrm{D}}{ }^{25}=+231.9\left(c 1.0, \mathrm{CHCl}_{3}\right) ;{ }^{1} \mathrm{H} \mathrm{NMR}\left(400 \mathrm{MHz}, \mathrm{CDCl}_{3}\right): \delta(\mathrm{ppm})$ 7.49-7.40 (m, 3H), 7.37-7.34 (m, 2H), 7.15-7.12 (m, 2H), 5.12-4.98 (m, br, 2H), $3.68(\mathrm{~d}, J=12.4 \mathrm{~Hz}, 1 \mathrm{H}), 1.53(\mathrm{~s}, 9 \mathrm{H}), 0.88(\mathrm{~d}, J=6.8 \mathrm{~Hz}, 3 \mathrm{H})$; ${ }^{13} \mathrm{C} \mathrm{NMR}\left(125 \mathrm{MHz}, \mathrm{CDCl}_{3}\right) \delta(\mathrm{ppm}) 162.5\left(\mathrm{~d}, J_{\mathrm{C}-\mathrm{F}}=246.3 \mathrm{~Hz}\right), 153.7,139.1,138.3$, $137.4,137.2,130.4,129.7,129.3,128.5,128.4,116.2,115.5\left(\mathrm{~d}, J_{\mathrm{C}-\mathrm{F}}=21.3 \mathrm{~Hz}\right), 80.1$, 57.7, 46.5, 28.7, 21.6. HRMS-ESI exact mass calcd. for $\mathrm{C}_{20} \mathrm{H}_{23} \mathrm{FNO}_{2}^{+}\left([\mathrm{M}+\mathrm{H}]^{+}\right)$ requires $\mathrm{m} / \mathrm{z}$ 328.1707, found $\mathrm{m} / \mathrm{z} 328.1710$.

The enantiomeric excess was determined by HPLC on Chiralcel IC column (hexane : isopropanol $=95: 5$, flowing rate $=1.0 \mathrm{~mL} / \mathrm{min}, 25^{\circ} \mathrm{C}$, $\mathrm{UV}$ detection at $\lambda=254 \mathrm{~nm}$ ), $\mathrm{t}_{\mathrm{R} 1}=4.9 \min$ (major), $\mathrm{t}_{\mathrm{R} 2}=5.6 \min$ (minor).

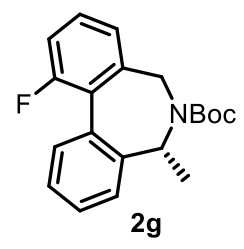

(+)-2g: (New compound); white solid, m.p. 110-111 ${ }^{\circ} \mathrm{C}, 31.0 \mathrm{mg}$, $95 \%$ yield, $90 \%$ ee, $[\alpha]_{\mathrm{D}}{ }^{25}=+274.3\left(c 1.0, \mathrm{CHCl}_{3}\right) ;{ }^{1} \mathrm{H} \mathrm{NMR}(500$ $\left.\mathrm{MHz}, \mathrm{CDCl}_{3}\right): \delta(\mathrm{ppm})$ 7.49-7.29 (m, 6H), 7.12-7.09 (m, 1H), $5.51(\mathrm{~s}$, br, 1H), 5.16 (s, br, 1H), $3.43(\mathrm{~d}, J=13.5 \mathrm{~Hz}, 1 \mathrm{H}), 1.54$ (s, 9H), 0.89 $(\mathrm{d}, J=6.5 \mathrm{~Hz}, 3 \mathrm{H}) ;{ }^{13} \mathrm{C} \mathrm{NMR}\left(125 \mathrm{MHz}, \mathrm{CDCl}_{3}\right) \delta(\mathrm{ppm}) 159.9\left(\mathrm{~d}, J_{\mathrm{C}-\mathrm{F}}=246.3 \mathrm{~Hz}\right)$, $153.6,143.8\left(\mathrm{~d}, J_{\mathrm{C}-\mathrm{F}}=3.8 \mathrm{~Hz}\right), 139.4,138.2,130.5,129.8,129.6\left(\mathrm{~d}, J_{\mathrm{C}-\mathrm{F}}=8.8 \mathrm{~Hz}\right)$, 
$128.8,128.4,123.3,122.5\left(\mathrm{~d}, J_{\mathrm{C}-\mathrm{F}}=17.5 \mathrm{~Hz}\right), 114.9\left(\mathrm{~d}, J_{\mathrm{C}-\mathrm{F}}=22.5 \mathrm{~Hz}\right), 80.0,57.6$, 38.4, 28.7, 21.4. HRMS-ESI exact mass calcd. for $\mathrm{C}_{20} \mathrm{H}_{23} \mathrm{FNO}_{2}{ }^{+}\left([\mathrm{M}+\mathrm{H}]^{+}\right)$requires $\mathrm{m} / \mathrm{z} 328.1707$, found $\mathrm{m} / \mathrm{z} 328.1709$.

The enantiomeric excess was determined by HPLC on Chiralcel IC column (hexane : isopropanol $=95: 5$, flowing rate $=1.0 \mathrm{~mL} / \mathrm{min}, 25{ }^{\circ} \mathrm{C}$, $\mathrm{UV}$ detection at $\lambda=254 \mathrm{~nm}$ ), $\mathrm{t}_{\mathrm{R} 1}=5.6 \mathrm{~min}$ (major), $\mathrm{t}_{\mathrm{R} 2}=6.5 \mathrm{~min}$ (minor).

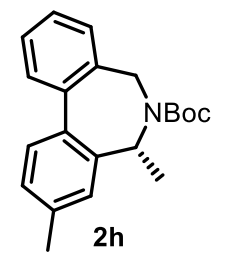

(+)-2h: (New compound); colorless oil, $31.0 \mathrm{mg}, 96 \%$ yield, $94 \%$ ee, $[\alpha]_{\mathrm{D}}{ }^{25}=+253.9\left(c 1.0, \mathrm{CHCl}_{3}\right) ;{ }^{1} \mathrm{H} \mathrm{NMR}\left(400 \mathrm{MHz}, \mathrm{CDCl}_{3}\right): \delta(\mathrm{ppm})$ 7.50-7.31 (m, 5H), $7.24(\mathrm{~d}, J=8.0 \mathrm{~Hz}, 1 \mathrm{H}), 7.16(\mathrm{~s}, 1 \mathrm{H}), 5.06(\mathrm{~s}, \mathrm{br}$, 2H), $3.71(\mathrm{~d}, J=12.8 \mathrm{~Hz}, 1 \mathrm{H}), 2.40(\mathrm{~s}, 3 \mathrm{H}), 1.53(\mathrm{~s}, 9 \mathrm{H}), 0.85(\mathrm{~d}, J=$ $6.8 \mathrm{~Hz}, 3 \mathrm{H}) ;{ }^{13} \mathrm{C} \mathrm{NMR}\left(100 \mathrm{MHz}, \mathrm{CDCl}_{3}\right) \delta(\mathrm{ppm}) 153.8,141.2,139.2,138.1,136.4$, 135.4, 131.0, 129.7, 129.3, 129.0, 128.6, 128.0, 127.6, 79.8, 57.7, 47.1, 28.8, 21.6, 21.2. HRMS-ESI exact mass calcd. for $\mathrm{C}_{21} \mathrm{H}_{26} \mathrm{O}_{2} \mathrm{~N}^{+}\left([\mathrm{M}+\mathrm{H}]^{+}\right)$requires $\mathrm{m} / \mathrm{z} 324.1958$, found $\mathrm{m} / \mathrm{z} 324.1960$.

The enantiomeric excess was determined by HPLC on Chiralcel IC column (hexane : isopropanol $=95: 5$, flowing rate $=1.0 \mathrm{~mL} / \mathrm{min}, 25^{\circ} \mathrm{C}$, $\mathrm{UV}$ detection at $\lambda=254 \mathrm{~nm}$ ), $\mathrm{t}_{\mathrm{R} 1}=5.0 \mathrm{~min}$ (major), $\mathrm{t}_{\mathrm{R} 2}=6.2 \mathrm{~min}$ (minor)

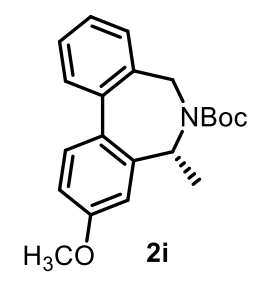

(+)-2i: (New compound); colorless oil, $33.0 \mathrm{mg}, 95 \%$ yield, 93\% ee, $[\alpha]_{\mathrm{D}}{ }^{25}=+308.8\left(c 1.0, \mathrm{CHCl}_{3}\right) ;{ }^{1} \mathrm{H} \mathrm{NMR}\left(500 \mathrm{MHz}, \mathrm{CDCl}_{3}\right): \delta(\mathrm{ppm})$ 7.48-7.31 (m, 5H), 6.98-6.96 (m, 1H), 6.90 (s, 1H), 5.04 (s, br, 1H), $3.87(\mathrm{~s}, 3 \mathrm{H}), 3.73(\mathrm{~d}, J=6.5 \mathrm{~Hz}, 1 \mathrm{H}), 1.54(\mathrm{~s}, 9 \mathrm{H}), 0.88(\mathrm{~d}, J=7.0 \mathrm{~Hz}$, $3 \mathrm{H}) ;{ }^{13} \mathrm{C} \mathrm{NMR}\left(125 \mathrm{MHz}, \mathrm{CDCl}_{3}\right) \delta$ (ppm) 159.6, 153.8, 140.9, 140.6, 135.2, 131.7, $130.9,129.2,128.6,127.7,127.4,115.7,113.5,79.9,58.0,55.5,47.7,46.8,28.7,21.6$. HRMS-ESI exact mass calcd. for $\mathrm{C}_{21} \mathrm{H}_{26} \mathrm{NO}_{3}{ }^{+}\left([\mathrm{M}+\mathrm{H}]^{+}\right)$requires $\mathrm{m} / \mathrm{z} 340.1907$, found $\mathrm{m} / \mathrm{z} 340.1906$.

The enantiomeric excess was determined by HPLC on Chiralcel IC column (hexane : isopropanol $=95: 5$, flowing rate $=1.0 \mathrm{~mL} / \mathrm{min}, 25^{\circ} \mathrm{C}$, $\mathrm{UV}$ detection at $\lambda=254 \mathrm{~nm}$ ), $\mathrm{t}_{\mathrm{R} 1}=6.1 \min ($ major $), \mathrm{t}_{\mathrm{R} 2}=7.5 \min ($ minor $)$. 


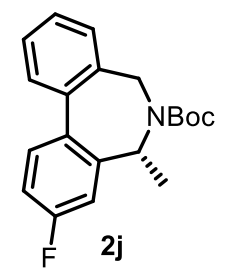

(+)-2j: (New compound); colorless oil, $31.0 \mathrm{mg}$, 95\% yield, $91 \%$ ee, $[\alpha]_{\mathrm{D}}{ }^{25}=+256.3\left(c 1.0, \mathrm{CHCl}_{3}\right) ;{ }^{1} \mathrm{H} \mathrm{NMR}\left(400 \mathrm{MHz}, \mathrm{CDCl}_{3}\right): \delta(\mathrm{ppm})$ 7.48-7.34 (m, 5H), 7.15-7.07 (m, 2H), 5.08 (s, br, 2H), 3.70 (d, $J=13.6$ $\mathrm{Hz}, 1 \mathrm{H}), 1.53(\mathrm{~s}, 9 \mathrm{H}), 0.86(\mathrm{~d}, J=6.8 \mathrm{~Hz}, 3 \mathrm{H}) ;{ }^{13} \mathrm{C}$ NMR $(100 \mathrm{MHz}$, $\left.\mathrm{CDCl}_{3}\right) \delta(\mathrm{ppm}) 162.5\left(\mathrm{~d}, J_{\mathrm{C}-\mathrm{F}}=246.0 \mathrm{~Hz}\right), 153.7,141.3\left(\mathrm{~d}, J_{\mathrm{C}-\mathrm{F}}=7.0 \mathrm{~Hz}\right), 140.2$, $135.3,131.4\left(\mathrm{~d}, J_{\mathrm{C}-\mathrm{F}}=8.0 \mathrm{~Hz}\right), 129.4,128.8,128.4,127.6,117.1\left(\mathrm{~d}, J_{\mathrm{C}-\mathrm{F}}=21.0 \mathrm{~Hz}\right)$, $115.1\left(\mathrm{~d}, J_{\mathrm{C}-\mathrm{F}}=21.0 \mathrm{~Hz}\right), 80.1,57.4,46.9,28.7,21.3$. HRMS-ESI exact mass calcd. for $\mathrm{C}_{20} \mathrm{H}_{23} \mathrm{FNO}_{2}{ }^{+}\left([\mathrm{M}+\mathrm{H}]^{+}\right)$requires $\mathrm{m} / \mathrm{z} 328.1707$, found $\mathrm{m} / \mathrm{z} 328.1708$.

The enantiomeric excess was determined by HPLC on Chiralcel IC column (hexane : isopropanol $=95: 5$, flowing rate $=1.0 \mathrm{~mL} / \mathrm{min}, 25^{\circ} \mathrm{C}$, $\mathrm{UV}$ detection at $\lambda=254 \mathrm{~nm}$ ), $\mathrm{t}_{\mathrm{R} 1}=5.1 \min$ (major), $\mathrm{t}_{\mathrm{R} 2}=5.7$ min (minor).

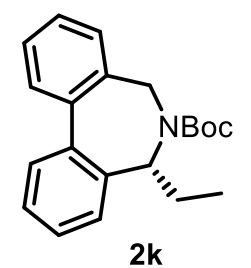

(+)-2k: (New compound); yellow oil, $29.0 \mathrm{mg}$, 90\% yield, $81 \%$ ee, $[\alpha]_{\mathrm{D}}{ }^{25}=+242.8\left(c 0.5, \mathrm{CHCl}_{3}\right) ;{ }^{1} \mathrm{H} \mathrm{NMR}\left(400 \mathrm{MHz}, \mathrm{CDCl}_{3}\right): \delta(\mathrm{ppm})$ 7.50-7.26 (m, 8H), 5.05-4.79 (m, br, 2H), 3.73 (s, br, 1H), 1.53 (s, $10 \mathrm{H}), 0.84$ (s, br, 1H), 0.44 (t, $J=7.2 \mathrm{~Hz}, 3 \mathrm{H}) ;{ }^{13} \mathrm{C}$ NMR $(100 \mathrm{MHz}$, $\left.\mathrm{CDCl}_{3}\right) \delta(\mathrm{ppm}) 153.9,141.3,139.2,137.3,135.3,131.8,129.5,128.7,128.4,128.2$, 127.9, 127.6, 79.8, 64.4, 63.7, 47.7, 46.6, 28.7, 27.5, 11.3. HRMS-ESI exact mass calcd. for $\mathrm{C}_{21} \mathrm{H}_{26} \mathrm{O}_{2} \mathrm{~N}^{+}\left([\mathrm{M}+\mathrm{H}]^{+}\right)$requires $\mathrm{m} / \mathrm{z} 324.1958$, found $\mathrm{m} / \mathrm{z} 324.1954$.

The enantiomeric excess was determined by HPLC on Chiralcel IC column (hexane : isopropanol $=95: 5$, flowing rate $=1.0 \mathrm{~mL} / \mathrm{min}, 25{ }^{\circ} \mathrm{C}$, UV detection at $\lambda=254 \mathrm{~nm}$ ), $\mathrm{t}_{\mathrm{R} 1}=4.8 \min$ (major), $\mathrm{t}_{\mathrm{R} 2}=5.8 \min$ (minor)

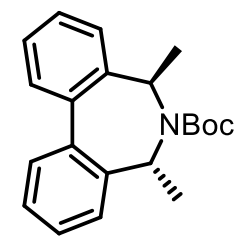

(a $R, 5 R, 7 R)-2 \mathbf{l}$ : (Known compound, see: Pira, S. L.; Wallace, T. W.; Graham, J. P. Org. Lett. 2009, 11, 1663); colorless oil, 98\% yield, $76 \%$ ee, trans $/$ cis $=1.3: 1 \mathrm{dr},[\alpha]_{\mathrm{D}}{ }^{25}=+183.8\left(\right.$ c $\left.0.5, \mathrm{CHCl}_{3}\right) ;{ }^{1} \mathrm{H} \mathrm{NMR}$ (aR,5R,7R)-2। $\quad\left(300 \mathrm{MHz}, \mathrm{CDCl}_{3}\right): \delta(\mathrm{ppm}) 7.50-7.33(\mathrm{~m}, 8 \mathrm{H}), 5.40(\mathrm{~s}, \mathrm{br}, 1 \mathrm{H}), 5.20$ (s, br, 1H), $1.53(\mathrm{~s}, 9 \mathrm{H}), 0.89\left(\mathrm{~d}, J_{1}=7.2 \mathrm{~Hz}, 6 \mathrm{H}\right) ;{ }^{13} \mathrm{C} \mathrm{NMR}\left(100 \mathrm{MHz}, \mathrm{CDCl}_{3}\right) \delta$ (ppm). 153.7, 146.9, 139.5, 130.1, 129.1, 128.5, 128.2, 85.3, 56.8, 27.6, 20.9, 20.5. 
The enantiomeric excess was determined by HPLC on Chiralcel IC column (hexane : isopropanol $=99: 1$, flowing rate $=0.5 \mathrm{~mL} / \mathrm{min}, 25^{\circ} \mathrm{C}$, $\mathrm{UV}$ detection at $\lambda=254 \mathrm{~nm}$ ), $\mathrm{t}_{\mathrm{R} 1}=11.8 \min ($ major$), \mathrm{t}_{\mathrm{R} 2}=14.3 \min ($ minor $)$

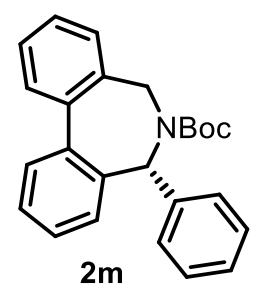

(+)-2m: (Known compound, see: Cheetham, C. A.; Massey, R. S.; Pira, S. L.; Pritchard, R. G.; Wallace, T. W. Org. Biomol. Chem. 2011, 9, 1831); white solid, m.p. $60-61{ }^{\circ} \mathrm{C}, 36.0 \mathrm{mg}, 97 \%$ yield, $80 \%$ ee, $[\alpha]_{\mathrm{D}}{ }^{25}=+219.5\left(c 1.0, \mathrm{CHCl}_{3}\right) ;{ }^{1} \mathrm{H} \mathrm{NMR}\left(300 \mathrm{MHz}, \mathrm{CDCl}_{3}\right): \delta$ (ppm) 7.56-7.36 (m, 5H), 7.22-7.11 (m, 2H), 7.05-7.02 (m, 1H), $6.85(\mathrm{~s}, 3 \mathrm{H}), 6.55$ (s, 2H), 6.28 (s, br, 1H), 5.14 (s, br, 1H), 3.89 (d, $J=12.9 \mathrm{~Hz}, 1 \mathrm{H}), 1.57-1.43$ (m, 9H); ${ }^{13} \mathrm{C}$ NMR $\left(100 \mathrm{MHz}, \mathrm{CDCl}_{3}\right) \delta(\mathrm{ppm}) 154.6,142.5,140.9,140.1,138.4,134.8,131.2$, $129.9,129.3,128.7,128.4,128.1,127.9,127.8,127.1,125.5,125.4,80.3,64.6,63.0$, 48.1, 47.2, 28.5. HRMS-ESI exact mass calcd. for $\mathrm{C}_{25} \mathrm{H}_{26} \mathrm{NO}_{2}{ }^{+}\left([\mathrm{M}+\mathrm{H}]^{+}\right)$requires $\mathrm{m} / \mathrm{z}$ 372.1958 , found $\mathrm{m} / \mathrm{z} 372.1956$.

The enantiomeric excess was determined by HPLC on Chiralcel IC column (hexane : isopropanol $=95: 5$, flowing rate $=1.0 \mathrm{~mL} / \mathrm{min}, 25{ }^{\circ} \mathrm{C}$, UV detection at $\lambda=254 \mathrm{~nm}$ ), $\mathrm{t}_{\mathrm{R} 1}=5.7$ min (major), $\mathrm{t}_{\mathrm{R} 2}=7.5 \min ($ minor $)$

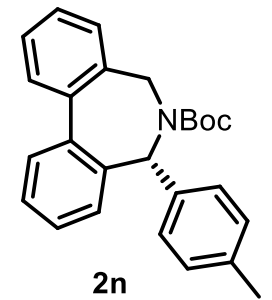

(+)-2n: (New compound); colorless oil, $35.0 \mathrm{mg}, 91 \%$ yield, $68 \%$ ee, $[\alpha]_{\mathrm{D}}{ }^{25}=+177.6\left(c\right.$ 1.0, $\left.\mathrm{CHCl}_{3}\right) ;{ }^{1} \mathrm{H} \mathrm{NMR}\left(400 \mathrm{MHz}, \mathrm{CDCl}_{3}\right): \delta(\mathrm{ppm})$ 7.55-7.40 (m, 5H), 7.24-7.14 (m, 2H), $7.07(\mathrm{~d}, J=7.6 \mathrm{~Hz}, 1 \mathrm{H}), 6.67$ $(\mathrm{d}, J=7.6 \mathrm{~Hz}, 2 \mathrm{H}), 6.44(\mathrm{~d}, J=8.0 \mathrm{~Hz}, 2 \mathrm{H}), 6.21$ (s, br, 1H), 5.19 (s, br, 1H), $3.90(\mathrm{~d}, J=11.6 \mathrm{~Hz}, 1 \mathrm{H}), 2.10(\mathrm{~s}, 3 \mathrm{H}), 1.55-1.40(\mathrm{~m}, 9 \mathrm{H}) ;{ }^{13} \mathrm{C}$ NMR $(100$ $\left.\mathrm{MHz}, \mathrm{CDCl}_{3}\right) \delta(\mathrm{ppm}) 154.6,140.9,140.0,139.5,138.6,134.8,131.1,129.9,129.2$, $128.6,128.3,128.0,127.9,127.8,127.8,125.3,80.2,64.5,47.1,28.5,20.9$. HRMS-ESI exact mass calcd. for $\mathrm{C}_{26} \mathrm{H}_{28} \mathrm{NO}_{2}{ }^{+}\left([\mathrm{M}+\mathrm{H}]^{+}\right)$requires $\mathrm{m} / \mathrm{z} 386.2115$, found $\mathrm{m} / \mathrm{z} 386.2117$.

The enantiomeric excess was determined by HPLC on Chiralcel IC column (hexane : isopropanol $=95: 5$, flowing rate $=1.0 \mathrm{~mL} / \mathrm{min}, 25{ }^{\circ} \mathrm{C}$, $\mathrm{UV}$ detection at $\lambda=254 \mathrm{~nm}$ ), $\mathrm{t}_{\mathrm{R} 1}=6.2 \min ($ major $), \mathrm{t}_{\mathrm{R} 2}=8.8 \min ($ minor $)$ 


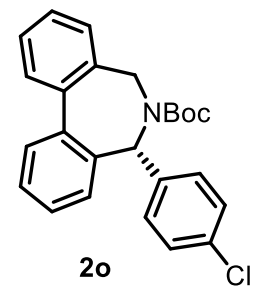

(+)-2o: (New compound); colorless oil, $40.0 \mathrm{mg}, 98 \%$ yield, $77 \%$ ee, $[\alpha]_{\mathrm{D}}{ }^{25}=+158.0\left(\right.$ ( $\left.1.0, \mathrm{CHCl}_{3}\right) ;{ }^{1} \mathrm{H} \mathrm{NMR}\left(400 \mathrm{MHz}, \mathrm{CDCl}_{3}\right): \delta(\mathrm{ppm})$ 7.55-7.41 (m, 5H), 7.24-7.15 (m, 2H), $7.05(\mathrm{~d}, J=7.2 \mathrm{~Hz}, 1 \mathrm{H}), 6.82$ (d, $J=8.4 \mathrm{~Hz}, 2 \mathrm{H}), 6.48(\mathrm{~d}, J=8.0 \mathrm{~Hz}, 2 \mathrm{H}), 6.37-6.16(\mathrm{~m}, \mathrm{br}, 1 \mathrm{H})$, 5.19-5.02 (m, br, 1H), 3.87 (s, br, 1H), 1.56-1.38 (m, 9H); ${ }^{13} \mathrm{C}$ NMR (100 MHz, $\left.\mathrm{CDCl}_{3}\right) \delta$ (ppm) 154.4, 141.3, 140.6, 140.0, 137.9, 134.8, 131.2, 131.1, 130.0, 129.3, 129.0, 128.6, 128.2, 127.8, 127.3, 126.8, 80.5, 64.1, 62.8, 48.0, 47.0, 28.5. HRMS-ESI exact mass calcd. for $\mathrm{C}_{25} \mathrm{H}_{25} \mathrm{ClNO}_{2}{ }^{+}\left([\mathrm{M}+\mathrm{H}]^{+}\right)$requires $\mathrm{m} / \mathrm{z}$ 406.1568, found $\mathrm{m} / \mathrm{z} 406.1570$.

The enantiomeric excess was determined by HPLC on Chiralcel IC column (hexane : isopropanol $=99: 1$, flowing rate $=1.0 \mathrm{~mL} / \mathrm{min}, 25^{\circ} \mathrm{C}, \mathrm{UV}$ detection at $\lambda=254 \mathrm{~nm}$ ), $\mathrm{t}_{\mathrm{R} 1}=7.9 \min$ (major), $\mathrm{t}_{\mathrm{R} 2}=9.7 \mathrm{~min}$ (minor)

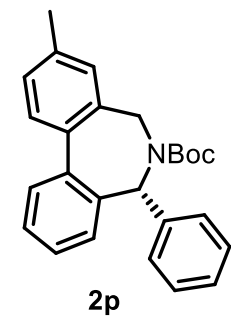

(+)-2p: (New compound); colorless oil, $36.0 \mathrm{mg}, 94 \%$ yield, $81 \%$ ee, $[\alpha]_{\mathrm{D}}{ }^{25}=+168.6\left(c 1.0, \mathrm{CHCl}_{3}\right) ;{ }^{1} \mathrm{H} \mathrm{NMR}\left(400 \mathrm{MHz}, \mathrm{CDCl}_{3}\right): \delta(\mathrm{ppm})$ 7.57-7.56 (m, 1H), 7.47-7.38 (m, 3H), 7.23 (s, br, 1H), 6.95-6.87 (m, $5 \mathrm{H}), 6.58(\mathrm{~s}, \mathrm{br}, 2 \mathrm{H}), 6.39-6.22(\mathrm{~m}, \mathrm{br}, 1 \mathrm{H}), 5.16-5.01(\mathrm{~m}, \mathrm{br}, 1 \mathrm{H})$, $3.89(\mathrm{~d}, J=12.8 \mathrm{~Hz}, 1 \mathrm{H}), 2.34(\mathrm{~s}, 3 \mathrm{H}), 1.54-1.28(\mathrm{~m}, 9 \mathrm{H}) ;{ }^{13} \mathrm{C}$ NMR $\left(100 \mathrm{MHz}, \mathrm{CDCl}_{3}\right) \delta(\mathrm{ppm}) 154.6,142.7,142.5,140.1,138.3,138.0,137.8,134.8$, 131.1, 129.9, 129.0, 128.7, 127.8, 127.6, 127.1, 125.5, 80.2, 64.8, 47.2, 28.5, 21.1. HRMS-ESI exact mass calcd. for $\mathrm{C}_{26} \mathrm{H}_{28} \mathrm{NO}_{2}^{+}\left([\mathrm{M}+\mathrm{H}]^{+}\right)$requires $\mathrm{m} / \mathrm{z} 386.2115$, found $\mathrm{m} / \mathrm{z} 386.2117$.

The enantiomeric excess was determined by HPLC on Chiralcel IC column (hexane : isopropanol $=95: 5$, flowing rate $=1.0 \mathrm{~mL} / \mathrm{min}, 25^{\circ} \mathrm{C}, \mathrm{UV}$ detection at $\lambda=254 \mathrm{~nm}$ ), $\mathrm{t}_{\mathrm{R} 1}=5.4$ min (major), $\mathrm{t}_{\mathrm{R} 2}=8.6 \min ($ minor $)$

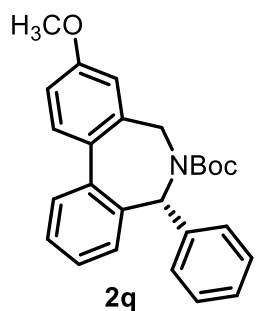

(+)-2q: (New compound); white solid, m.p. $94-95{ }^{\circ} \mathrm{C}, 35.0 \mathrm{mg}, 87 \%$ yield, $60 \%$ ee, $[\alpha]_{\mathrm{D}}{ }^{25}=+151.2\left(c 1.0, \mathrm{CHCl}_{3}\right) ;{ }^{1} \mathrm{H}$ NMR $(500 \mathrm{MHz}$, $\left.\mathrm{CDCl}_{3}\right): \delta(\mathrm{ppm}) 7.55(\mathrm{~s}, \mathrm{br}, 1 \mathrm{H}), 7.46-7.39(\mathrm{~m}, 2 \mathrm{H}), 7.36(\mathrm{~d}, J=7.5$ 
$\mathrm{Hz}, 1 \mathrm{H}), 6.98-6.88(\mathrm{~m}, 5 \mathrm{H}), 6.69$ (d, $J=8.0 \mathrm{~Hz}, 1 \mathrm{H}), 6.59-6.58(\mathrm{~m}, 2 \mathrm{H}), 6.43-6.20$ (m, br, 1H), 5.16-4.97 (m, br, 1H), 3.88 (s, br, 1H), $3.81(\mathrm{~s}, 3 \mathrm{H}), 1.57-1.37(\mathrm{~m}, 9 \mathrm{H}) ;{ }^{13} \mathrm{C}$ NMR (125 MHz, $\left.\mathrm{CDCl}_{3}\right) \delta(\mathrm{ppm}) 159.5,154.6,142.7,142.0,139.9,138.1,136.2$, 133.5, 131.1, 129.7, 129.0, 128.7, 127.5, 125.6, 125.4, 114.3, 113.4, 80.3, 64.8, 63.3, 55.6, 48.3, 47.2, 28.5. HRMS-ESI exact mass calcd. for $\mathrm{C}_{26} \mathrm{H}_{28} \mathrm{NO}_{3}{ }^{+}\left([\mathrm{M}+\mathrm{H}]^{+}\right)$ requires $\mathrm{m} / \mathrm{z}$ 402.2064, found $\mathrm{m} / \mathrm{z} 402.2065$.

The enantiomeric excess was determined by HPLC on Chiralcel IC column (hexane : isopropanol $=95: 5$, flowing rate $=1.0 \mathrm{~mL} / \mathrm{min}, 25^{\circ} \mathrm{C}$, UV detection at $\lambda=254 \mathrm{~nm}$ ), $\mathrm{t}_{\mathrm{R} 1}=6.7$ min (major), $\mathrm{t}_{\mathrm{R} 2}=11.8 \mathrm{~min}$ (minor)

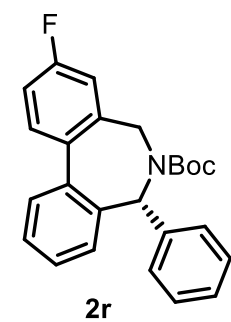

(+)-2r: (New compound); white solid, m.p. $45-46{ }^{\circ} \mathrm{C}, 38.0 \mathrm{mg}, 97 \%$ yield, $77 \%$ ee, $[\alpha]_{\mathrm{D}}{ }^{25}=+166.8\left(c 1.0, \mathrm{CHCl}_{3}\right) ;{ }^{1} \mathrm{H} \mathrm{NMR}(500 \mathrm{MHz}$, $\left.\mathrm{CDCl}_{3}\right): \delta(\mathrm{ppm})$ 7.58-7.36 (m, 4H), 7.12-6.81 (m, 6H), 6.58-6.56 (m, $2 \mathrm{H}), 6.42-6.23(\mathrm{~m}, \mathrm{br}, 1 \mathrm{H}), 5.17-5.00(\mathrm{~m}, \mathrm{br}, 1 \mathrm{H}), 3.86(\mathrm{~s}, \mathrm{br}, 1 \mathrm{H})$, 1.56-1.39 (m, 9H); ${ }^{13} \mathrm{C}$ NMR (125 MHz, $\left.\mathrm{CDCl}_{3}\right) \delta(\mathrm{ppm}) 162.3\left(\mathrm{~d}, J_{\mathrm{C}-\mathrm{F}}\right.$ = 246.3 Hz), 154.5, 142.3, 139.2, 138.3, 137.0, 131.2, 129.8, 129.5, 128.8, 128.2, $127.3,125.7,125.3,116.0\left(\mathrm{~d}, J_{\mathrm{C}-\mathrm{F}}=21.3 \mathrm{~Hz}\right), 115.2\left(\mathrm{~d}, J_{\mathrm{C}-\mathrm{F}}=21.3 \mathrm{~Hz}\right), 80.5,64.5$, 63.0, 47.8, 46.8, 28.5. HRMS-ESI exact mass calcd. for $\mathrm{C}_{25} \mathrm{H}_{25} \mathrm{FNO}_{2}^{+}\left([\mathrm{M}+\mathrm{H}]^{+}\right)$ requires $\mathrm{m} / \mathrm{z} 390.1864$, found $\mathrm{m} / \mathrm{z} 390.1865$.

The enantiomeric excess was determined by HPLC on Chiralcel IC column (hexane : isopropanol $=95: 5$, flowing rate $=1.0 \mathrm{~mL} / \mathrm{min}, 25^{\circ} \mathrm{C}$, $\mathrm{UV}$ detection at $\lambda=254 \mathrm{~nm}$ ), $\mathrm{t}_{\mathrm{R} 1}=5.2 \min ($ major $), \mathrm{t}_{\mathrm{R} 2}=6.6 \mathrm{~min}$ (minor)

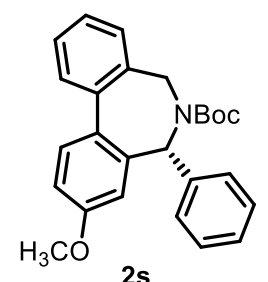

(+)-2s: (New compound); colorless oil, $34.0 \mathrm{mg}, 87 \%$ yield, $70 \%$ ee, $[\alpha]_{\mathrm{D}}{ }^{25}=+191.5\left(c 1.0, \mathrm{CHCl}_{3}\right) ;{ }^{1} \mathrm{H} \mathrm{NMR}\left(500 \mathrm{MHz}, \mathrm{CDCl}_{3}\right): \delta(\mathrm{ppm})$ 7.34-7.33 (m, 2H), 7.16-7.09 (m, 3H), 7.02-6.99 (m, 2H), 6.87-6.86 (m, 3H), 6.59-6.58 (m, 2H), 6.38-6.15 (m, br, 1H), 5.19-5.01 (m, br, 1H), $3.93(\mathrm{~m}, 4 \mathrm{H}), 1.57-1.37$ (m, br, 9H); $\left.{ }^{13} \mathrm{C} \mathrm{NMR} \mathrm{(125} \mathrm{MHz,} \mathrm{CDCl}_{3}\right) \delta(\mathrm{ppm})$ 159.5, $154.6,142.4$, 140.7, 139.6, 134.7, 132.5, 131.0, 129.3, 128.3, 127.5, 127.4, 127.1, 125.6, 125.5, 117.0, 114.1, 113.4, 80.3, 64.7, 63.2, 55.6, 48.3, 47.2, 29.8, 28.5. 
HRMS-ESI exact mass calcd. for $\mathrm{C}_{26} \mathrm{H}_{28} \mathrm{NO}_{3}{ }^{+}\left([\mathrm{M}+\mathrm{H}]^{+}\right)$requires $\mathrm{m} / \mathrm{z} 402.2064$, found $\mathrm{m} / \mathrm{z} 402.2062$.

The enantiomeric excess was determined by HPLC on Chiralcel IC column (hexane : isopropanol $=95: 5$, flowing rate $=1.0 \mathrm{~mL} / \mathrm{min}, 25{ }^{\circ} \mathrm{C}$, $\mathrm{UV}$ detection at $\lambda=254 \mathrm{~nm}$ ), $\mathrm{t}_{\mathrm{R} 1}=6.2 \min$ (major), $\mathrm{t}_{\mathrm{R} 2}=7.7$ min (minor).

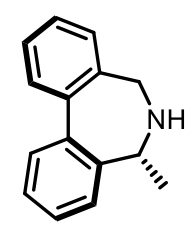

(aS,5R)-9: (Known compound, see: Pira, S. L.; Wallace, T. W.; Graham, J. P. Org. Lett. 2009, 11, 1663); yellow oil, $387.0 \mathrm{mg}$, 95\% yield, 93\% ee, $[\alpha]_{\mathrm{D}}^{25}=-33.6\left(c 0.5, \mathrm{CHCl}_{3}\right)$, Lit. $^{2}[\alpha]_{\mathrm{D}}^{26}=+26.4\left(c 1.38, \mathrm{CHCl}_{3}\right), 99 \%$ (aS,5R)-9 ee for $(S)$ enantiomer]; ${ }^{1} \mathrm{H}$ NMR $\left(400 \mathrm{MHz}, \mathrm{CDCl}_{3}\right): \delta(\mathrm{ppm})$ 7.49-7.33 (m, 8H), 3.74-3.69 (m, 2H), $3.50(\mathrm{~d}, J=12.4 \mathrm{~Hz}, 1 \mathrm{H}), 2.14(\mathrm{~s}, 1 \mathrm{H}), 1.47(\mathrm{~d}, J=6.4$ $\mathrm{Hz}, 3 \mathrm{H}) ;{ }^{13} \mathrm{C}$ NMR $\left(125 \mathrm{MHz}, \mathrm{CDCl}_{3}\right) \delta(\mathrm{ppm}) 141.3,141.3,139.5,137.2,128.4$, $128.2,128.2,128.1,128.0,127.8,127.6,125.0,50.3,49.6,19.1$.

For the secondary amine product, direct determination of its ee value was difficult. To facilitate the measurement, the obtained chiral amine was derived into the N-Boc product $(\mathrm{a} R, 5 R)-\mathbf{2 a}$ with $(\mathrm{Boc})_{2} \mathrm{O}$.

The enantiomeric excess was determined by HPLC on Chiralcel IC column (hexane : isopropanol $=95: 5$, flowing rate $=1.0 \mathrm{~mL} / \mathrm{min}, 25^{\circ} \mathrm{C}$, $\mathrm{UV}$ detection at $\lambda=254 \mathrm{~nm}$ ), $\mathrm{t}_{\mathrm{R} 1}=5.1 \min ($ major $), \mathrm{t}_{\mathrm{R} 2}=6.1 \mathrm{~min}$ (minor). 


\section{General procedure for the asymmetric reductive amination}

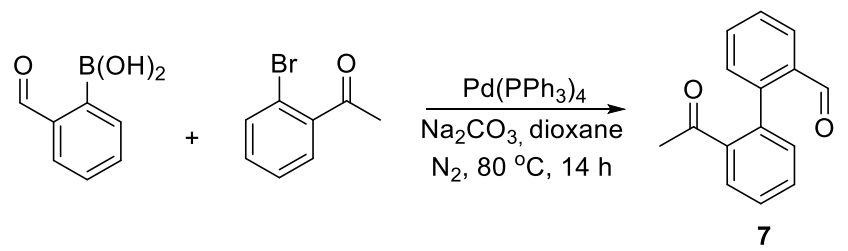

Typical procedure: ${ }^{4}$ 1-(2-bromophenyl)ethan-1-one $(1.3 \mathrm{~mL}, 10.0 \mathrm{mmol})$, (2-formylphenyl)boronic acid (1.7 g, $11.0 \mathrm{mmol}), \mathrm{Pd}\left(\mathrm{PPh}_{3}\right)_{4}(578 \mathrm{mg}, 5.0 \mathrm{~mol} \%)$ and $\mathrm{Na}_{2} \mathrm{CO}_{3}(5.7 \mathrm{~g}, 20 \mathrm{mmol})$ were added to a round bottom flask $(100 \mathrm{~mL})$. The mixture was suspended in dry-dioxane $(50 \mathrm{~mL})$ and stirred for $14 \mathrm{~h}$ at $80{ }^{\circ} \mathrm{C}$. The solvent was removed under reduced pressure and the crude product was subjected to column chromatography on silica gel to afford 7 (1.6 g, 71\% yield) as a yellow oil (eluent: petroleum ether/ethyl acetate $=30 / 1$ to $10 / 1$ ).<smiles>CC(=O)c1ccccc1-c1ccccc1C=O</smiles>

7: (Known compound, see: Choi, Y. L.; Yu, C.-M.; Kim, B. T.; Heo, J.-N. J. Org. Chem. 2009, 74, 3948); yellow oil; ${ }^{1} \mathrm{H}$ NMR (400 MHz, $\left.\mathrm{CDCl}_{3}\right): \delta(\mathrm{ppm}) 9.84(\mathrm{~s}, 1 \mathrm{H}), 8.01(\mathrm{~d}, J=7.6 \mathrm{~Hz}, 1 \mathrm{H}), 7.79(\mathrm{~d}, J=7.2$ $\mathrm{Hz}, 1 \mathrm{H}), 7.62-7.50(\mathrm{~m}, 4 \mathrm{H}), 7.29-7.24(\mathrm{~m}, 2 \mathrm{H}), 2.25(\mathrm{~s}, 3 \mathrm{H}) ;{ }^{13} \mathrm{C} \mathrm{NMR}$ (125 MHz, $\left.\mathrm{CDCl}_{3}\right): \delta(\mathrm{ppm}) 201.2,191.7,144.8,139.6,137.5,134.0,133.6,132.0$, $131.2,130.7,128.8,128.5,128.3,128.3,29.6$

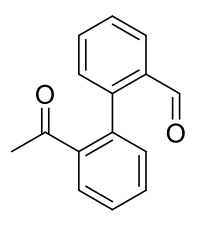

7

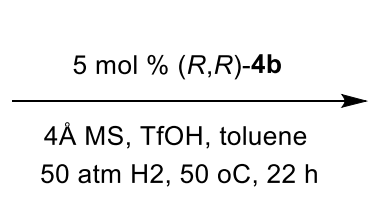

$0 \mathrm{~atm} \mathrm{H} 2,50$ oC, $22 \mathrm{~h}$

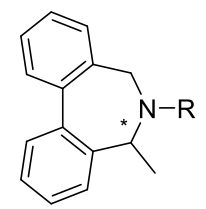

8

Typical procedure: A $30 \mathrm{~mL}$ glass-lined stainless-steel reactor equipped with a magnetic stirrer bar was charged with Ru-catalyst $(R, R)-\mathbf{4 b}(3.8 \mathrm{mg}, 5.0 \mathrm{~mol} \%), 7$ (0.1 mmol), $\mathrm{RNH}_{2}(0.1 \mathrm{mmol}), 4 \AA \mathrm{MS}(100 \mathrm{mg}), \mathrm{TfOH}(2.5 \mathrm{~mol} \%)$ in toluene $(1 \mathrm{~mL})$ under nitrogen atmosphere in a glove box. The autoclave was closed, and the final pressure of the hydrogen gas was adjusted to 50 atm after purging the autoclave with hydrogen gas several times. The reaction mixture was stirred at $50{ }^{\circ} \mathrm{C}$ for $22 \mathrm{~h}$. Then 
the hydrogen gas was carefully released and the conversion was determined by ${ }^{1} \mathrm{H}$ NMR. The reaction mixture was filtered through a short pad of silica (petroleum/ethyl acetate, 10/1, v/v) to give the pure products. The enantiomeric excess of the product was determined by HPLC with a chiral IA column.

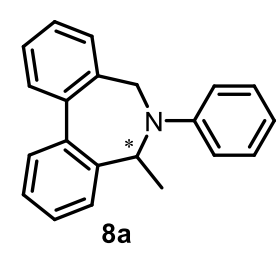

(+)-8a: (New compound); colorless oil, 91\% yield, $77 \%$ ee, $[\alpha]_{\mathrm{D}}^{25}$ $=+265.3\left(c 0.6, \mathrm{CHCl}_{3}\right) ;{ }^{1} \mathrm{H} \mathrm{NMR}\left(400 \mathrm{MHz}, \mathrm{CDCl}_{3}\right): \delta(\mathrm{ppm})$ 7.55-7.52 (m, 2H), 7.47-7.40 (m, 2H), 7.37-7.23 (m, 6H), 6.94 (d, $J$ $=8.4 \mathrm{~Hz}, 2 \mathrm{H}), 6.83-6.79(\mathrm{~m}, 1 \mathrm{H}), 4.78(\mathrm{q}, J=7.1 \mathrm{~Hz}, 1 \mathrm{H}), 4.32(\mathrm{~d}, J$ $=12.0 \mathrm{~Hz}, 1 \mathrm{H}), 3.87(\mathrm{~d}, J=12.4 \mathrm{~Hz}, 1 \mathrm{H}), 1.02(\mathrm{~d}, J=7.2 \mathrm{~Hz}, 3 \mathrm{H}) ;{ }^{13} \mathrm{C}$ NMR $(75$ $\left.\mathrm{MHz}, \mathrm{CDCl}_{3}\right) \delta(\mathrm{ppm}) 150.7,141.3,139.6,139.1,135.8,129.9,129.7,129.3,129.0$, 128.5, 128.3, 128.2, 128.1, 127.5, 118.3, 115.4, 62.8, 51.2, 21.9. HRMS-ESI exact mass calcd. for $\mathrm{C}_{21} \mathrm{H}_{20} \mathrm{~N}^{+}\left([\mathrm{M}+\mathrm{H}]^{+}\right)$requires $\mathrm{m} / \mathrm{z} 286.1590$, found $\mathrm{m} / \mathrm{z} 286.1586$.

The enantiomeric excess was determined by HPLC on Chiralcel IA column (hexane : isopropanol $=99.7: 0.3$, flowing rate $=0.4 \mathrm{~mL} / \mathrm{min}, 25^{\circ} \mathrm{C}$, $\mathrm{UV}$ detection at $\lambda=254$ $\mathrm{nm}), \mathrm{t}_{\mathrm{R} 1}=16.5 \min$ (major), $\mathrm{t}_{\mathrm{R} 2}=18.4 \min ($ minor $)$.

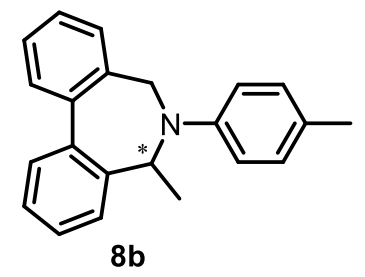

(+)-8b: (New compound); colorless oil, $80 \%$ yield, $83 \%$ ee, $[\alpha]_{\mathrm{D}}{ }^{25}=+318.4\left(c 0.5, \mathrm{CHCl}_{3}\right) ;{ }^{1} \mathrm{H} \mathrm{NMR}\left(400 \mathrm{MHz}, \mathrm{CDCl}_{3}\right): \delta$ (ppm) 7.54-7.23 (m, 8H), $7.08(\mathrm{~d}, J=8.0 \mathrm{~Hz}, 2 \mathrm{H}), 6.87$ (d, $J=$ $8.0 \mathrm{~Hz}, 2 \mathrm{H}), 4.66(\mathrm{q}, J=6.9 \mathrm{~Hz}, 1 \mathrm{H}), 4.22(\mathrm{~d}, J=12.0 \mathrm{~Hz}, 1 \mathrm{H})$, $3.84(\mathrm{~d}, J=12.0 \mathrm{~Hz}, 1 \mathrm{H}), 2.29(\mathrm{~s}, 3 \mathrm{H}), 1.04(\mathrm{~d}, J=6.8 \mathrm{~Hz}, 3 \mathrm{H}) ;{ }^{13} \mathrm{C} \mathrm{NMR}(100 \mathrm{MHz}$, $\left.\mathrm{CDCl}_{3}\right) \delta(\mathrm{ppm}) 148.9,141.3,139.6,139.4,135.8,129.7,129.5,128.9,128.4,128.2$, 128.1, 128.0, 127.4, 116.7, 62.5, 52.3, 21.8, 20.6. HRMS-ESI exact mass calcd. for $\mathrm{C}_{22} \mathrm{H}_{22} \mathrm{~N}^{+}\left([\mathrm{M}+\mathrm{H}]^{+}\right)$requires $\mathrm{m} / \mathrm{z} 300.1747$, found $\mathrm{m} / \mathrm{z} 300.1745$.

The enantiomeric excess was determined by HPLC on Chiralcel IA column (hexane : isopropanol $=99.7: 0.3$, flowing rate $=0.4 \mathrm{~mL} / \mathrm{min}, 25{ }^{\circ} \mathrm{C}$, $\mathrm{UV}$ detection at $\lambda=254$ $\mathrm{nm}), \mathrm{t}_{\mathrm{R} 1}=16.0 \min ($ major $), \mathrm{t}_{\mathrm{R} 2}=18.6 \min ($ minor $)$ 


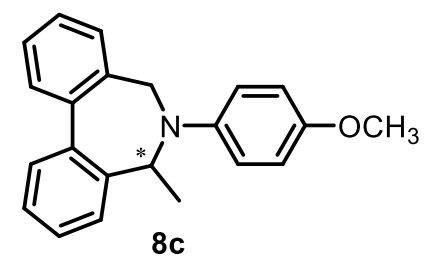

(+)-8c: (New compound); colorless oil, 76\% yield, 78\% ee, $[\alpha]_{\mathrm{D}}{ }^{25}=+167.0\left(c \quad 0.3, \mathrm{CHCl}_{3}\right) ;{ }^{1} \mathrm{H}$ NMR $(400 \mathrm{MHz}$, $\left.\mathrm{CDCl}_{3}\right): \delta(\mathrm{ppm}) \delta(\mathrm{ppm}) 7.55-7.24(\mathrm{~m}, 8 \mathrm{H}), 6.96(\mathrm{~d}, J=$ $8.0 \mathrm{~Hz}, 2 \mathrm{H}), 6.84(\mathrm{~d}, J=9.2 \mathrm{~Hz}, 2 \mathrm{H}), 4.42(\mathrm{q}, J=6.7 \mathrm{~Hz}$, $1 \mathrm{H}), 4.07(\mathrm{~d}, J=12.4 \mathrm{~Hz}, 1 \mathrm{H}), 3.83-3.79(\mathrm{~m}, 4 \mathrm{H}), 1.10(\mathrm{~d}, J=6.4 \mathrm{~Hz}, 3 \mathrm{H}) ;{ }^{13} \mathrm{C}$ NMR $\left(100 \mathrm{MHz}, \mathrm{CDCl}_{3}\right) \delta(\mathrm{ppm}) 154.4,145.4,141.2,139.9,139.3,135.5,129.6,128.7$, 128.6, 128.3, 128.2, 128.1, 127.9, 127.4, 120.9, 114.4, 61.1, 55.7, 54.8, 21.0. HRMS-ESI exact mass calcd. for $\mathrm{C}_{22} \mathrm{H}_{22} \mathrm{ON}^{+}\left([\mathrm{M}+\mathrm{H}]^{+}\right)$requires $\mathrm{m} / \mathrm{z} 316.1696$, found $\mathrm{m} / \mathrm{z} 316.1694$.

The enantiomeric excess was determined by HPLC on Chiralcel IA column (hexane : isopropanol $=99.7: 0.3$, flowing rate $=0.4 \mathrm{~mL} / \mathrm{min}, 25^{\circ} \mathrm{C}, \mathrm{UV}$ detection at $\lambda=254$ $\mathrm{nm}), \mathrm{t}_{\mathrm{R} 1}=22.5 \min$ (major), $\mathrm{t}_{\mathrm{R} 2}=26.8 \mathrm{~min}$ (minor).

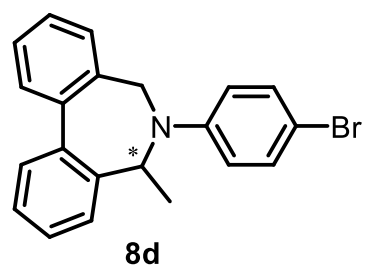

(+)-8d: (New compound); colorless oil, 55\% yield, $73 \%$ ee, $[\alpha]_{\mathrm{D}}^{25}=+285.0\left(\right.$ c $\left.0.5, \mathrm{CHCl}_{3}\right) ;{ }^{1} \mathrm{H} \mathrm{NMR}\left(400 \mathrm{MHz}, \mathrm{CDCl}_{3}\right)$ : $\delta(\mathrm{ppm})$ 7.55-7.52 (m, 2H), 7.48-7.41 (m, 2H), 7.37-7.30 (m, $5 \mathrm{H}), 7.24-7.21(\mathrm{~m}, 1 \mathrm{H}), 6.80(\mathrm{~d}, J=9.2 \mathrm{~Hz}, 2 \mathrm{H}), 4.71(\mathrm{q}, J=$ $7.1 \mathrm{~Hz}, 1 \mathrm{H}), 4.26(\mathrm{~d}, J=12.0 \mathrm{~Hz}, 1 \mathrm{H}), 3.84(\mathrm{~d}, J=12.0 \mathrm{~Hz}, 1 \mathrm{H}), 1.00(\mathrm{~d}, J=7.2 \mathrm{~Hz}$, $3 \mathrm{H}) ;{ }^{13} \mathrm{C}$ NMR $\left(100 \mathrm{MHz}, \mathrm{CDCl}_{3}\right) \delta(\mathrm{ppm})$ 149.6, 141.2, 139.3, 139.0, 135.4, 132.0, 129.8, 129.7, 129.1, 128.6, 128.3, 128.3, 128.3, 127.6, 116.9, 110.2, 62.9, 51.2, 21.7. HRMS-ESI exact mass calcd. for $\mathrm{C}_{21} \mathrm{H}_{19} \mathrm{NBr}^{+}\left([\mathrm{M}+\mathrm{H}]^{+}\right)$requires $\mathrm{m} / \mathrm{z} 364.0695$, found $\mathrm{m} / \mathrm{z} 364.0690$.

The enantiomeric excess was determined by HPLC on Chiralcel IA column (hexane : isopropanol $=99.7: 0.3$, flowing rate $=0.4 \mathrm{~mL} / \mathrm{min}, 25^{\circ} \mathrm{C}, \mathrm{UV}$ detection at $\lambda=254$ $\mathrm{nm}$ ), $\mathrm{t}_{\mathrm{R} 1}=18.8 \mathrm{~min}$ (major), $\mathrm{t}_{\mathrm{R} 2}=22.7 \mathrm{~min}$ (minor). 


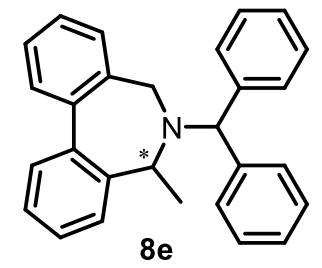

(+)-8e: (New compound); colorless oil, 53\% yield, 47\% ee, $[\alpha]_{\mathrm{D}}^{25}=+4.8\left(c 0.5, \mathrm{CHCl}_{3}\right) ;{ }^{1} \mathrm{H} \mathrm{NMR}\left(400 \mathrm{MHz}, \mathrm{CDCl}_{3}\right): \delta(\mathrm{ppm})$ $7.60(\mathrm{~d}, J=7.6 \mathrm{~Hz}, 2 \mathrm{H}), 7.53-7.14(\mathrm{~m}, 15 \mathrm{H}), 6.99(\mathrm{~d}, J=7.2 \mathrm{~Hz}$, $1 \mathrm{H}), 4.67(\mathrm{~s}, 1 \mathrm{H}), 4.00(\mathrm{q}, J=7.2 \mathrm{~Hz}, 1 \mathrm{H}), 3.58(\mathrm{~d}, J=11.2 \mathrm{~Hz}$, $1 \mathrm{H}), 2.83(\mathrm{~d}, J=11.2 \mathrm{~Hz}, 1 \mathrm{H}), 0.69(\mathrm{~d}, J=7.2 \mathrm{~Hz}, 3 \mathrm{H}) ;{ }^{13} \mathrm{C} \mathrm{NMR}\left(100 \mathrm{MHz}, \mathrm{CDCl}_{3}\right)$ $\delta(\mathrm{ppm}) 144.8,144.1,141.9,139.6,139.3,137.2,130.6,129.7,128.9,128.8,128.7$, $128.1,128.0,127.9,127.8,127.7,127.1,127.1,127.0,75.8,60.4,54.9,23.1$. HRMS-ESI exact mass calcd. for $\mathrm{C}_{28} \mathrm{H}_{26} \mathrm{~N}^{+}\left([\mathrm{M}+\mathrm{H}]^{+}\right)$requires $\mathrm{m} / \mathrm{z} 376.2060$, found $\mathrm{m} / \mathrm{z} 376.2057$.

The enantiomeric excess was determined by HPLC on Chiralcel IA column (hexane : isopropanol $=99.7: 0.3$, flowing rate $=0.4 \mathrm{~mL} / \mathrm{min}, 25^{\circ} \mathrm{C}$, $\mathrm{UV}$ detection at $\lambda=254$ $\mathrm{nm}), \mathrm{t}_{\mathrm{R} 1}=12.2 \min ($ minor $), \mathrm{t}_{\mathrm{R} 2}=16.1 \mathrm{~min}$ (major). 


\section{References}

1. (a) Haack, K.-J.; Hashiguchi, S.; Fujii, A.; Ikariya, T.; Noyori, R. Angew. Chem., Int. Ed. 1997, 36, 285. (b) Chen, F.; Wang, T.; Ding, Z.; He, Y.; Li, Z.; Xu, L.; Fan, Q.-H. Chem. Eur. J. 2011, 17, 1109.

2. France, S. P.; Aleku, G. A.; Sharma, M.; Mangas-Sanchez, J.; Howard, R. M.; Steflik, J.; Kumar, R.; Adams, R. W.; Slabu, I.; Crook, R.; Grogan, G.; Wallace, T. W.; Turner, N. J. Angew. Chem., Int. Ed. 2017, 56, 15589.

3. Cheetham, C. A.; Massey, R. S.; Pira, S. L.; Pritchard, R. G.; Wallace, T. W. Org. Biomol. Chem. 2011, 9, 1831.

4. Bhowmik, S.; Bhattacharyya, S.; Batra, S. Tetrahedron 2014, 70, 4031. 


\section{Copy of NMR spectra}
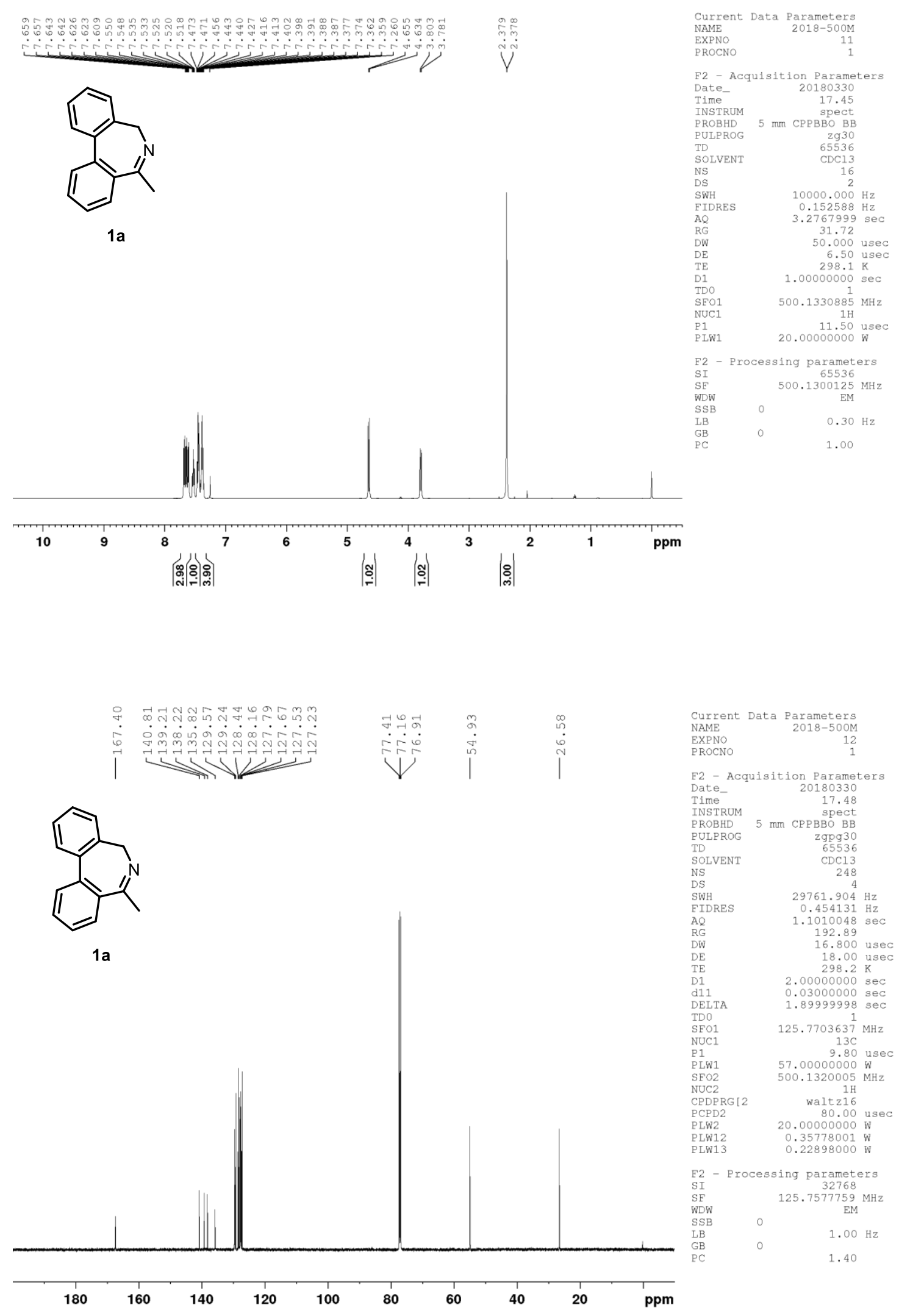


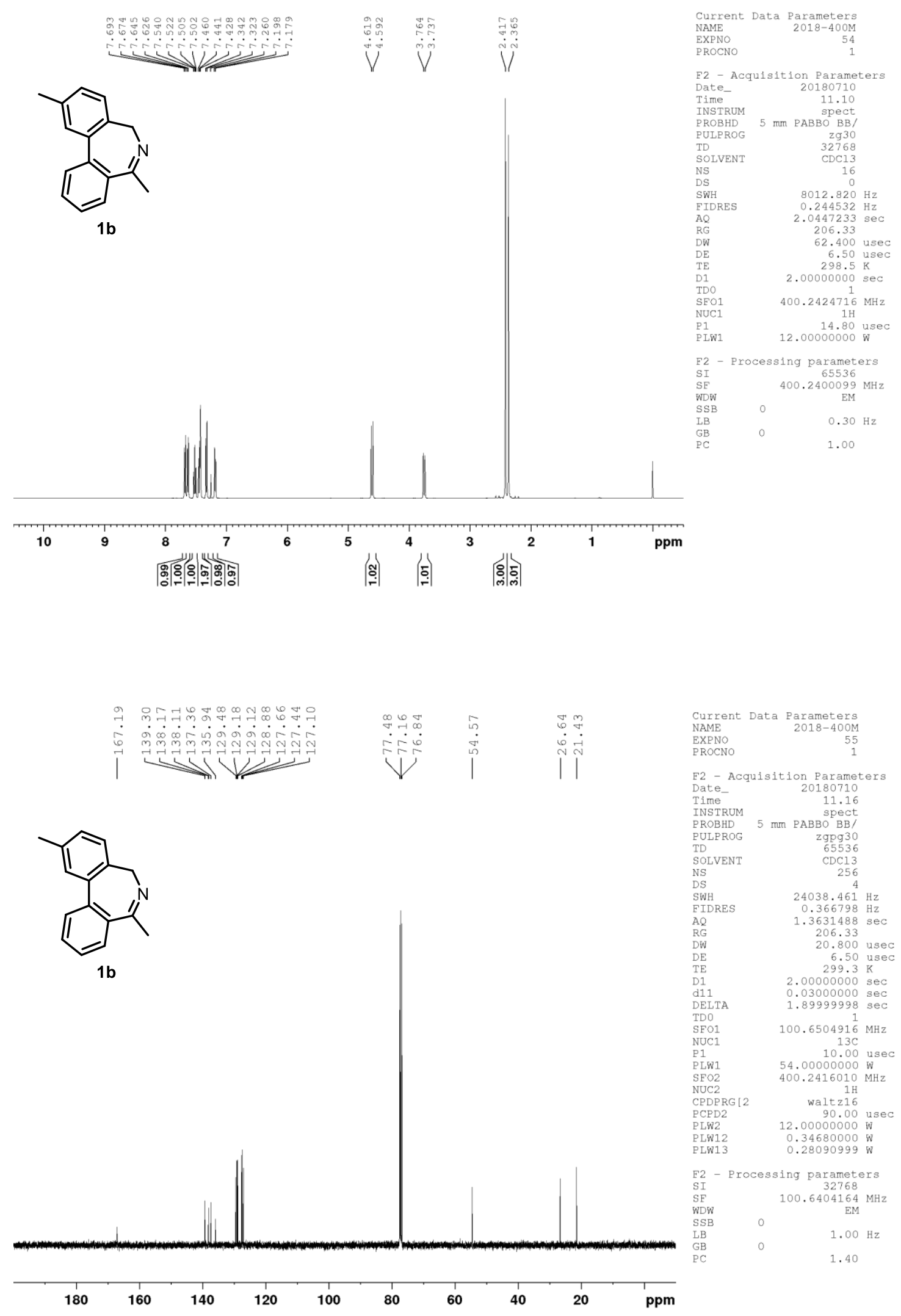



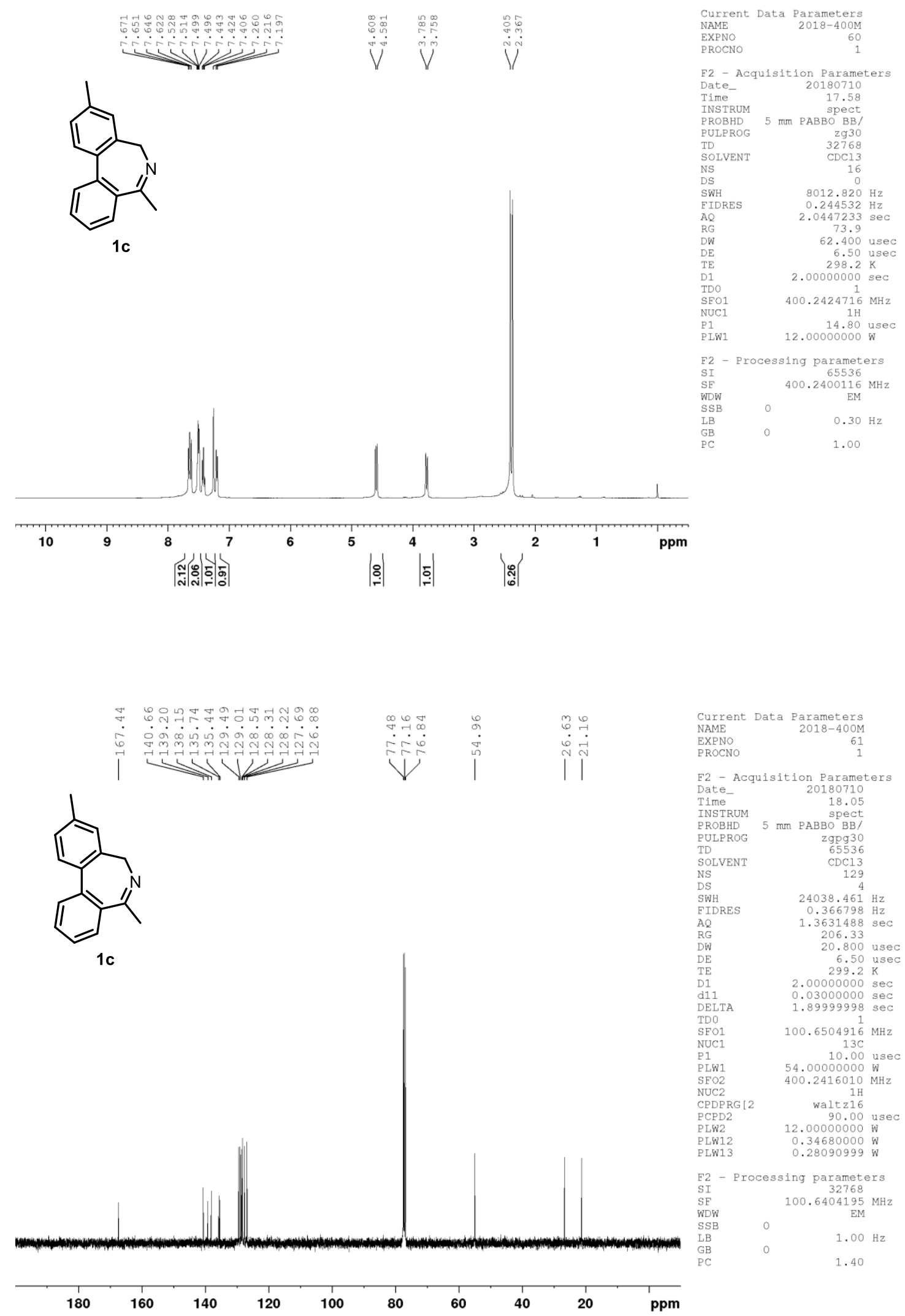


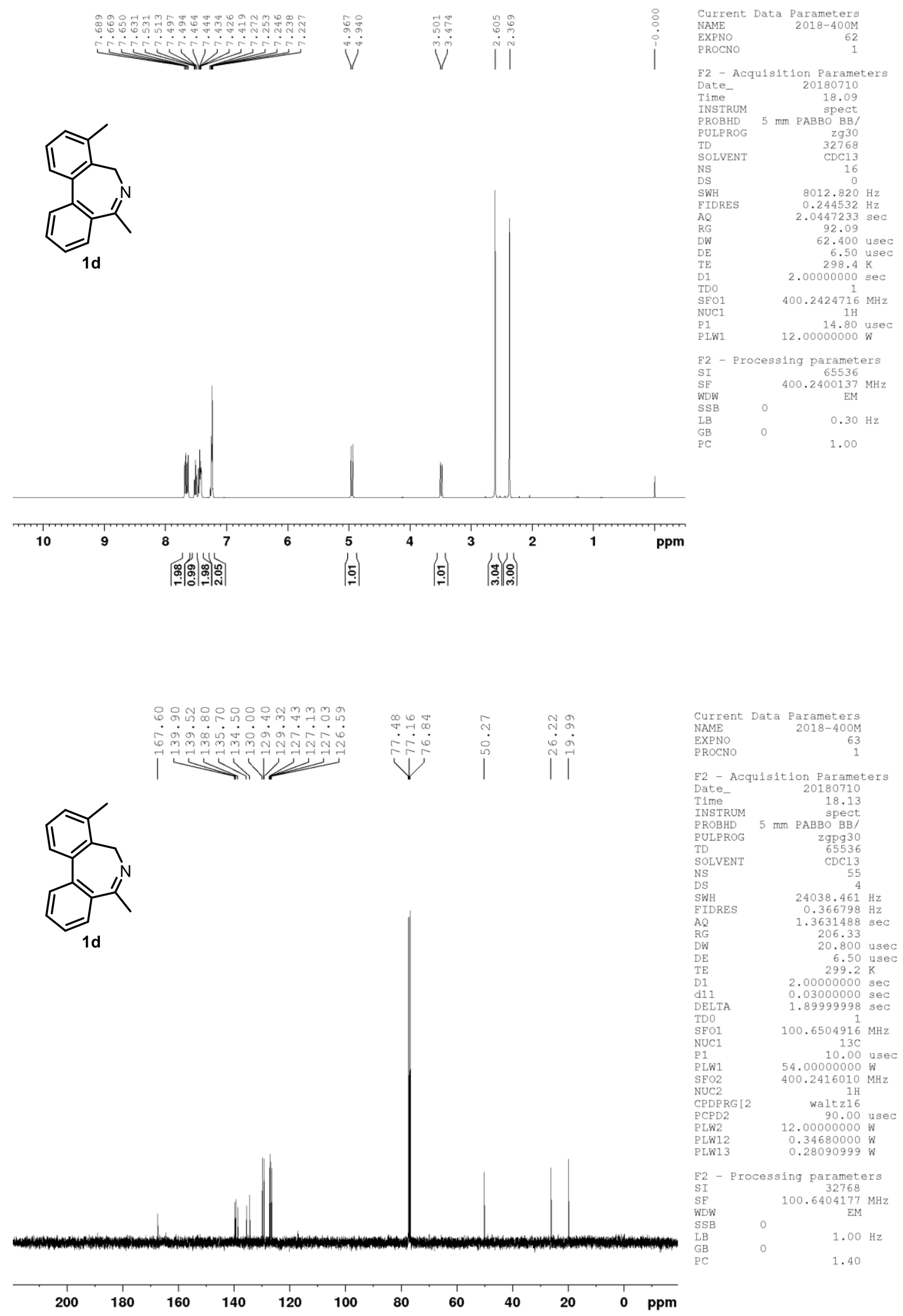




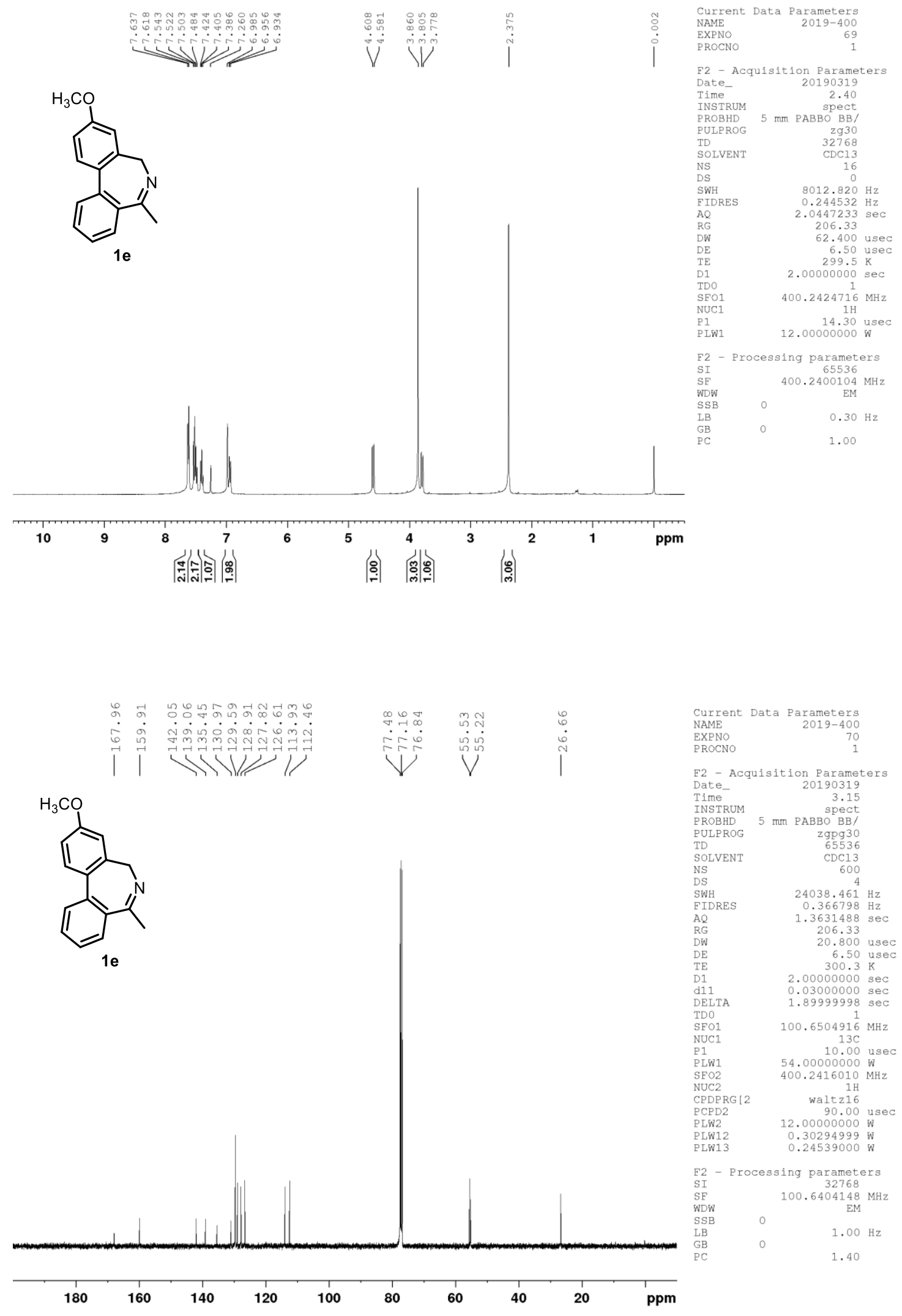




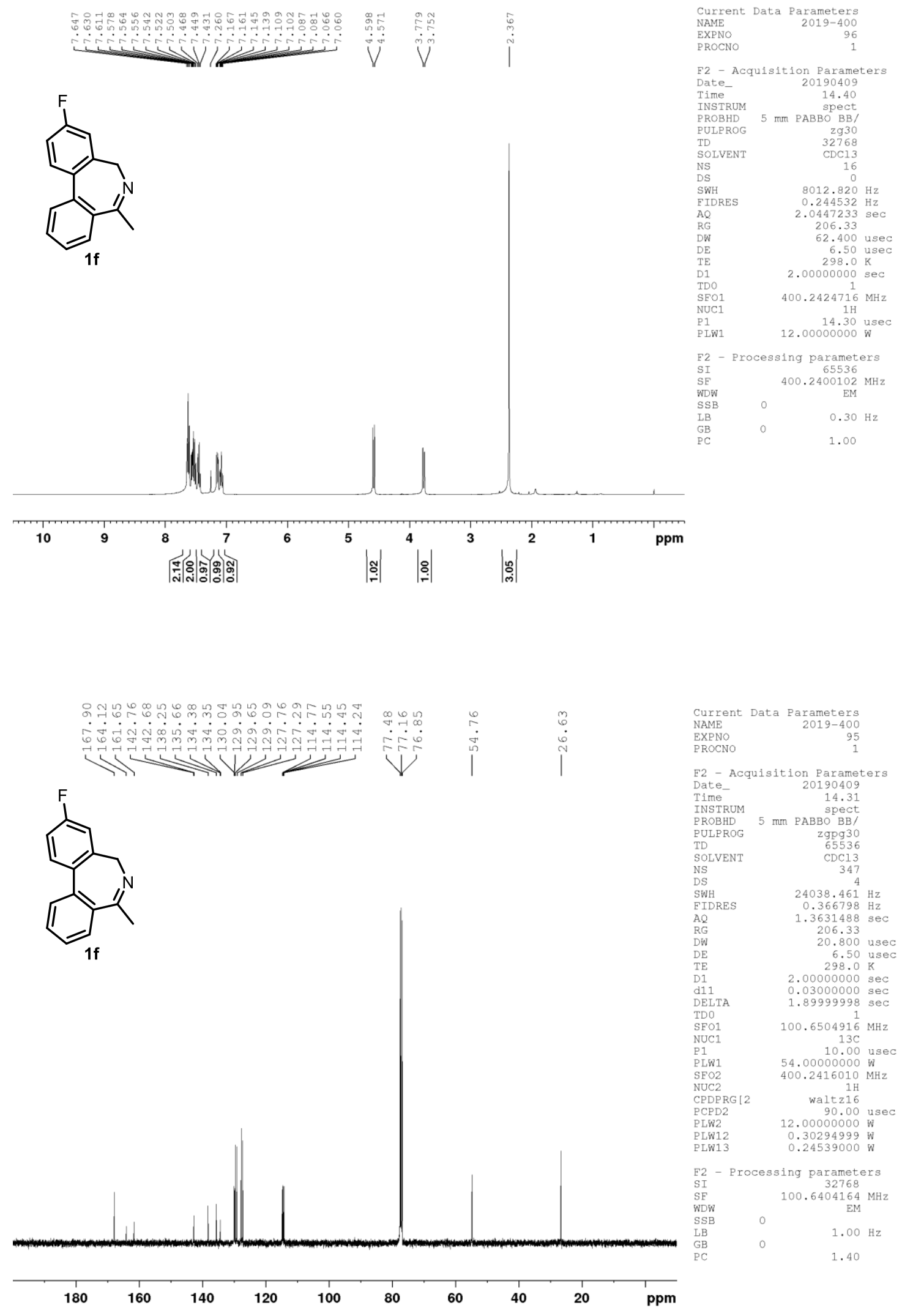




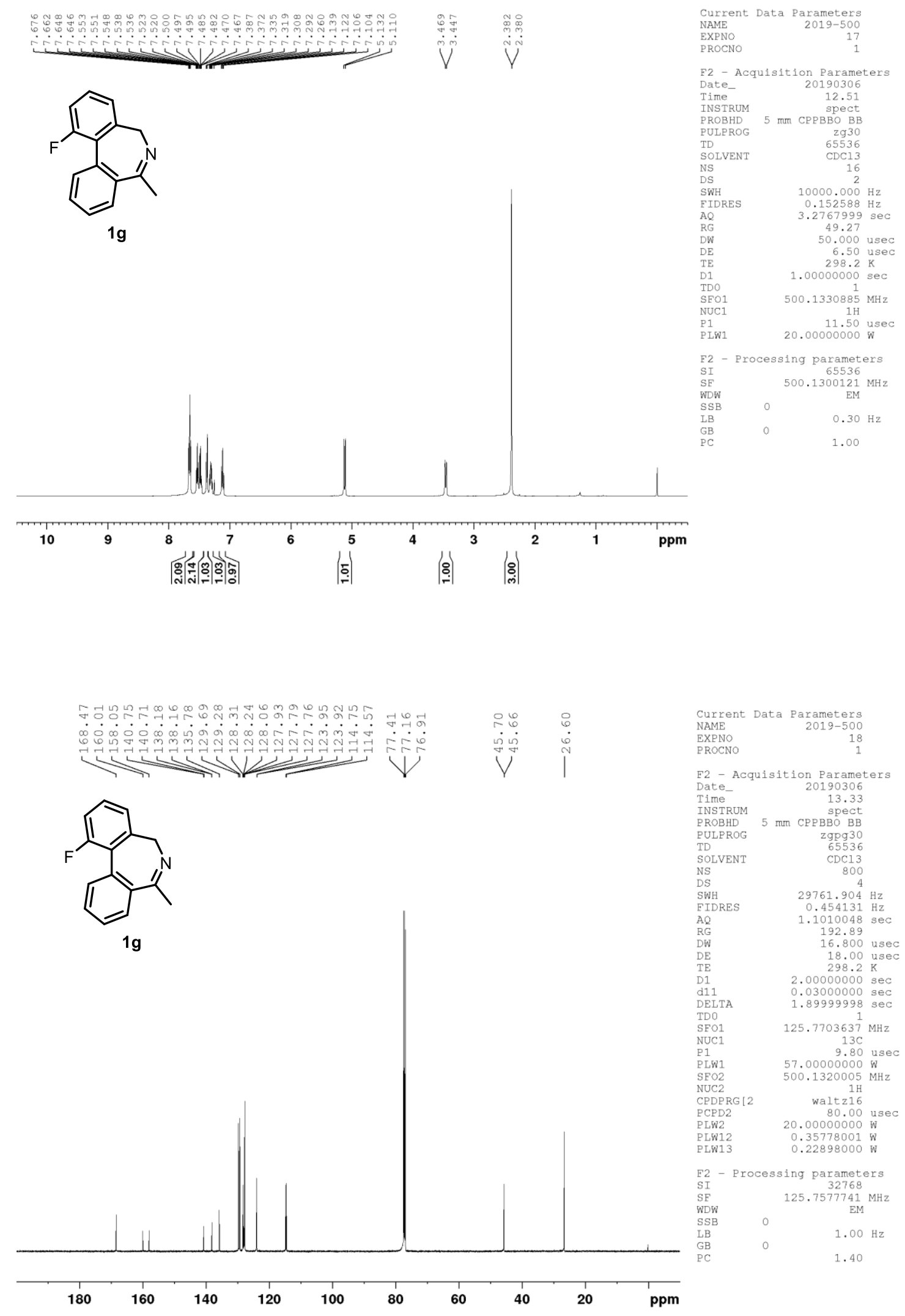




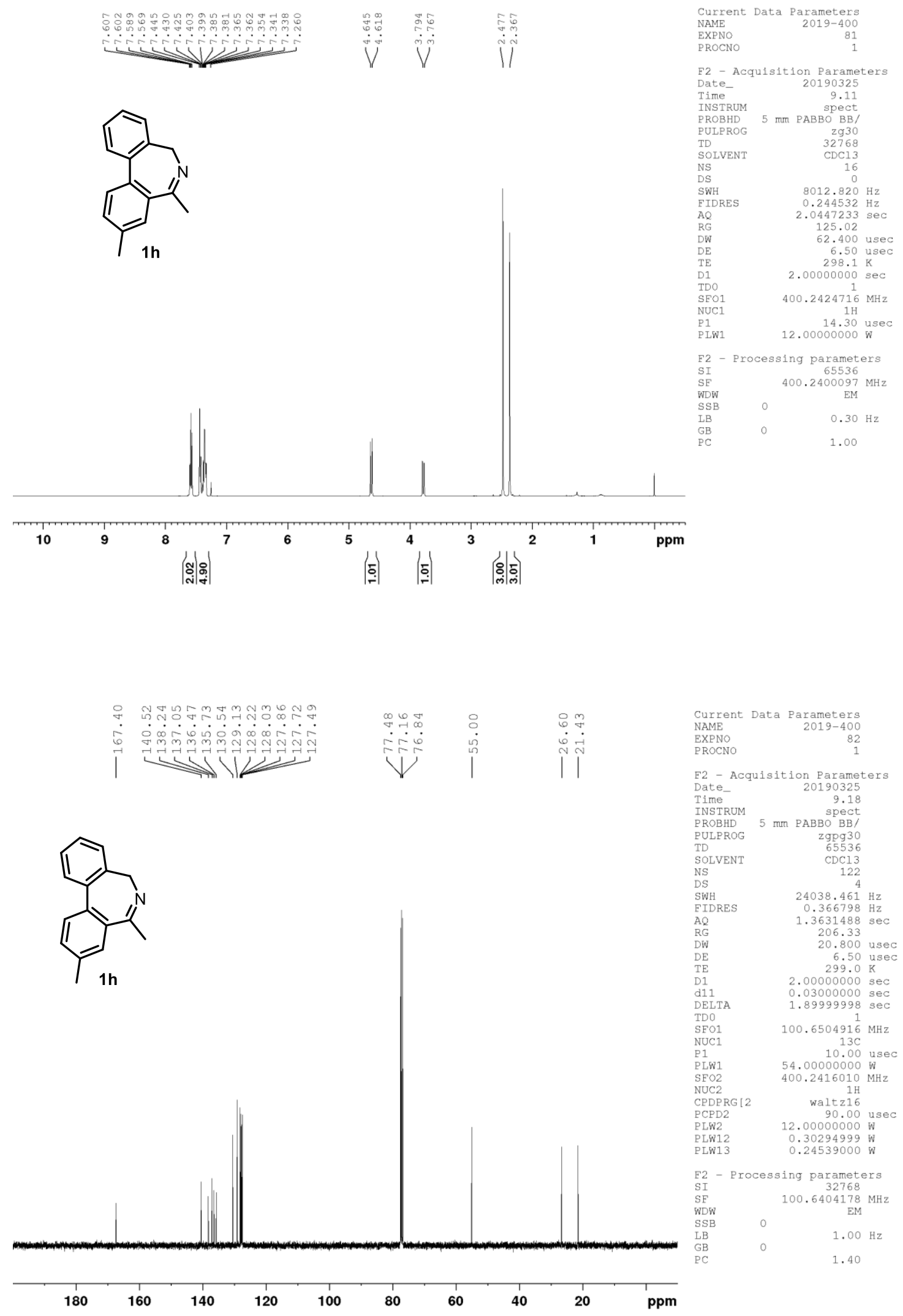




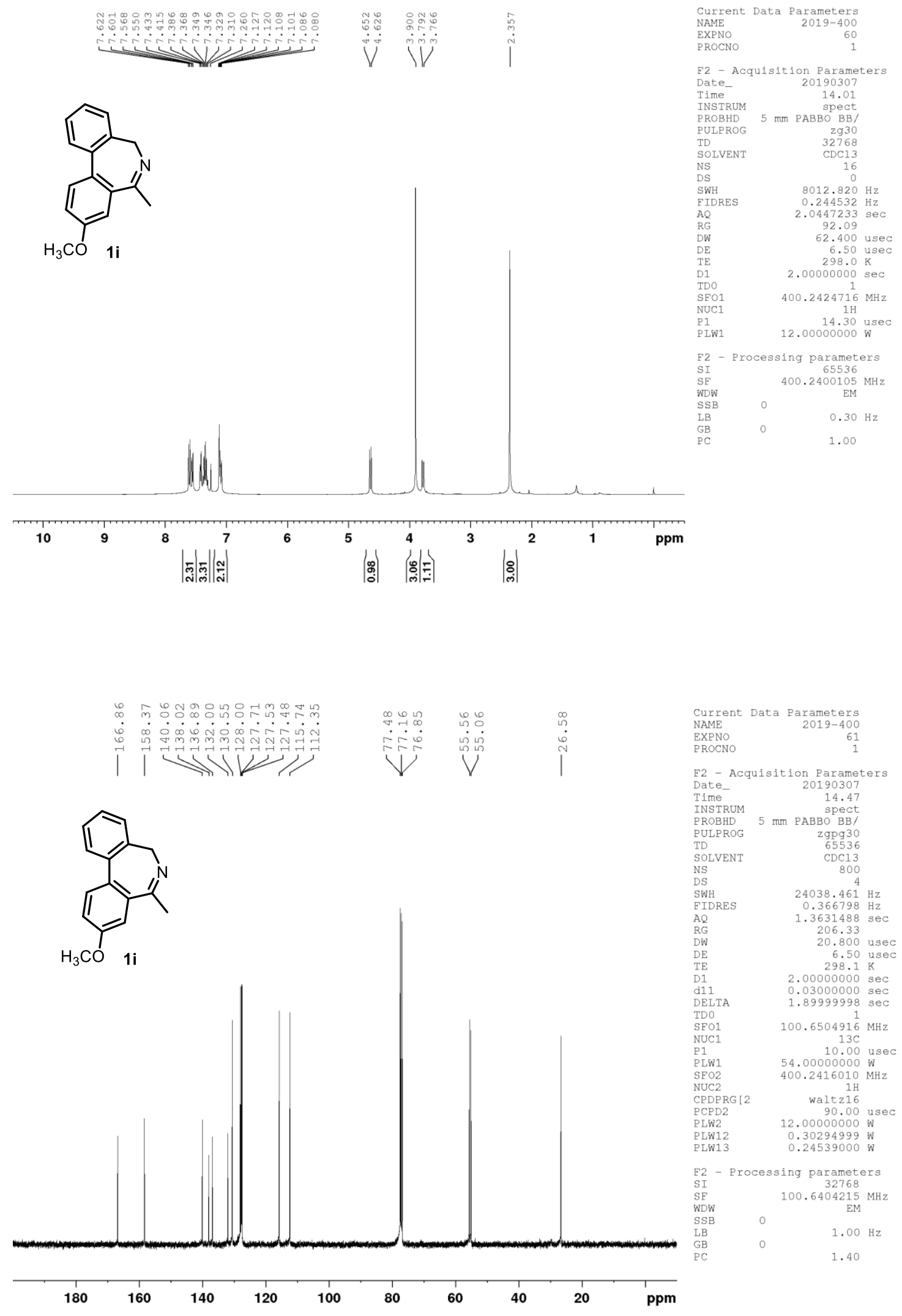




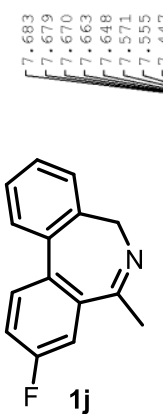

$\begin{array}{lr}\text { Current } & \text { Data } \\ \text { NAME } & 2019-400 \\ \text { EXPNO } & 71 \\ \text { PROCNO } & 1\end{array}$

F2 - Acquisition Parameters

Date_ 20190319

Time 3.18

INSTRUM
PROBHD $5 \mathrm{~mm}$ PABBO BB/

PROBHD $5 \mathrm{~mm}$ PABBO BB/
PULPROG
32306

$\begin{array}{lr}\text { PULPROG } & \mathrm{zg} 30 \\ \text { TD } & 32768\end{array}$

NS

$\begin{array}{ll}\text { SWH } & 0 \\ & 8012.820 \mathrm{~Hz}\end{array}$

FIDRES $0.244532 \mathrm{~Hz}$

$\begin{array}{lr}\text { AQ } & 2.0447233 \mathrm{sec} \\ \text { RG } & 206.33\end{array}$

$\begin{array}{lr}\text { DW } & 62.400 \text { usec } \\ \text { DE } & 6.50 \text { usec }\end{array}$

$\begin{array}{lr}\text { TE } & 299.6 \mathrm{~K} \\ \text { TE } & 2.0000000 \mathrm{sec}\end{array}$

IDO $\quad 400.2424716 \mathrm{MH}$

NEC1 $\quad 400.2424716 \mathrm{MH}$

$\begin{array}{ll}\text { P1 } & 14.30 \text { use } \\ \text { PLW1 } & 12.00000000 \mathrm{~W}\end{array}$

F2 - Processing parameters

$\begin{array}{lr}\text { SI } & 65536 \\ \text { SE } & 400.2400117 \mathrm{MHz}\end{array}$

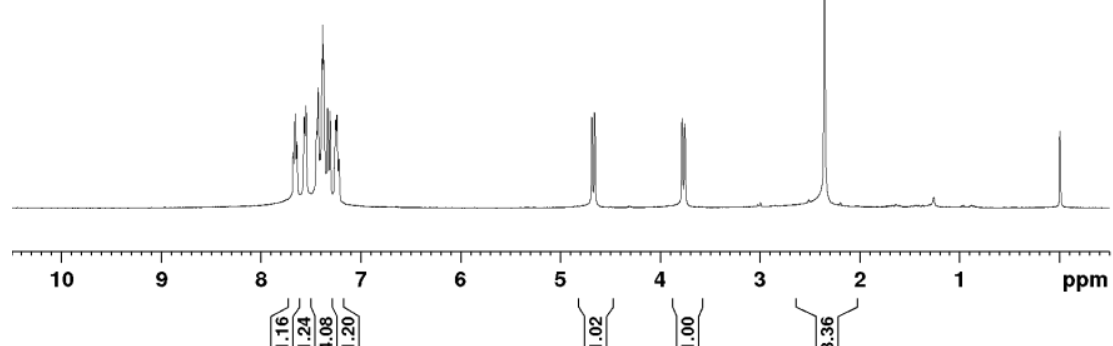

$\begin{array}{lll}\mathrm{SB} & 0.30 \mathrm{~Hz}\end{array}$

$\begin{array}{lll}\mathrm{GB} & 0 \\ \mathrm{PC} & 1.00\end{array}$

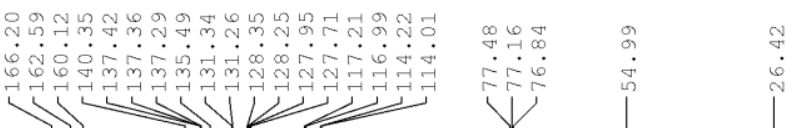<smiles>CC1=NCc2ccccc2-c2ccc(F)cc21</smiles>

$1 \mathrm{j}$

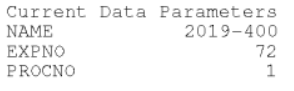

F2 - Acquisition Parameters

Date_r 20190319

INSTRUM spect

PROBHD $5 \mathrm{~mm}$ PABBO BB/
PULPROG
zgpg30

TD

SOLVENT

NS

$\begin{array}{lr}\text { SWH } & 24038.461 \mathrm{~Hz} \\ \text { FIDRES } & 0.366798 \mathrm{~Hz}\end{array}$

AQ $\quad 1.3631488 \mathrm{sec}$

$\begin{array}{ll}\text { RG } & 206.33 \\ \text { DW } & 20.800 \text { usec }\end{array}$

6.50 usec

$2.00000000 \mathrm{sec}$

$0.03000000 \mathrm{sec}$
$1.89999998 \mathrm{sec}$

$100.6504916 \mathrm{MHz}$

$13 \mathrm{C}$

54.00000000 use

$400.2416010 \mathrm{MHz}$

$$
\begin{array}{r}
1 \mathrm{H} \\
\text { waltzi6 }
\end{array}
$$

$12.00000000 \mathrm{~W}$

0.24539000 W

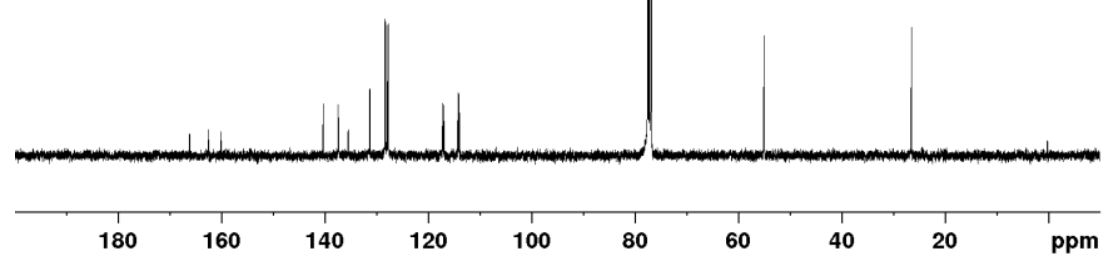

F2 - Processing parameters

SI $\quad 32768$ WDW 100.6404144 MH

$1.00 \mathrm{~Hz}$

1.40 

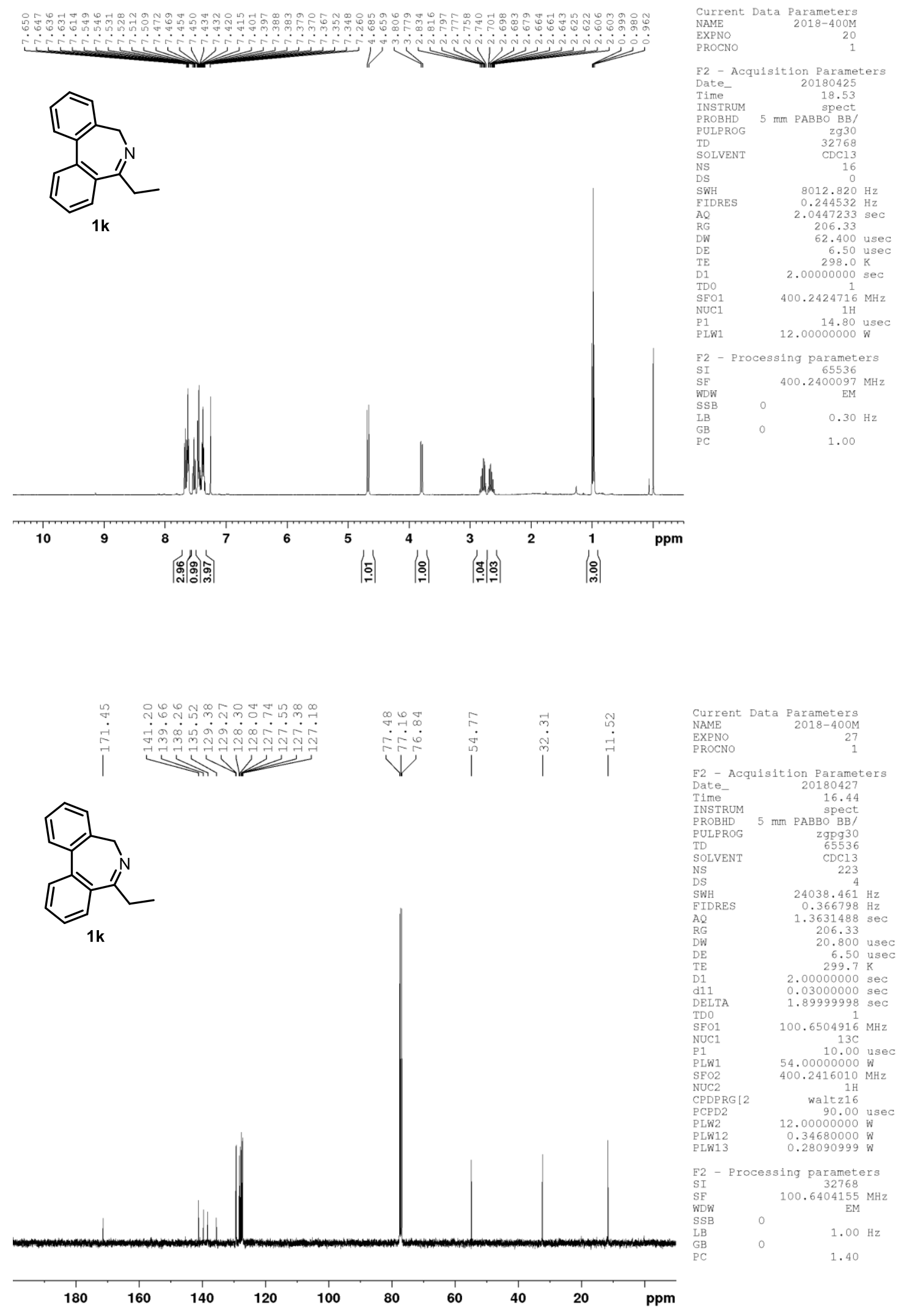


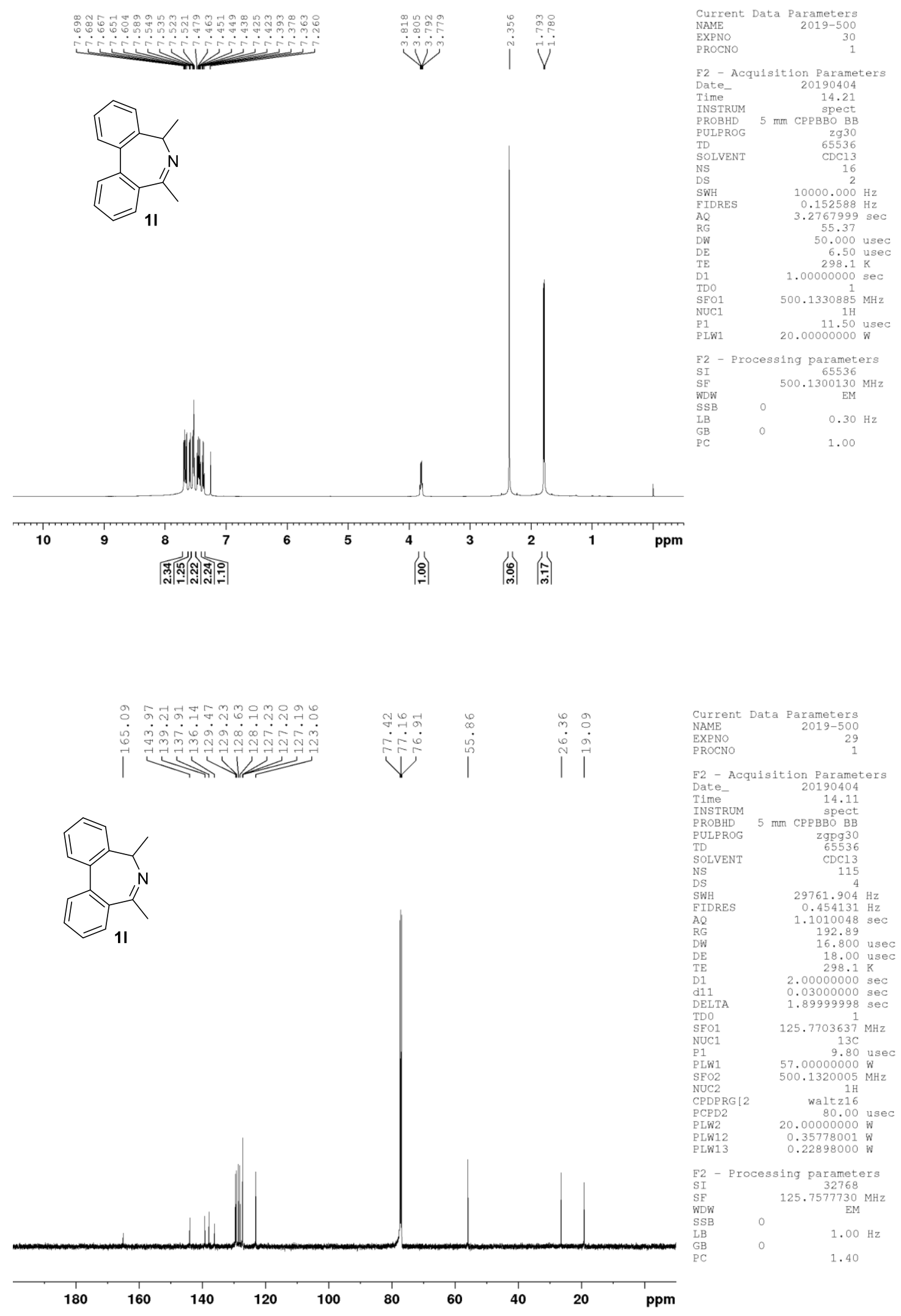



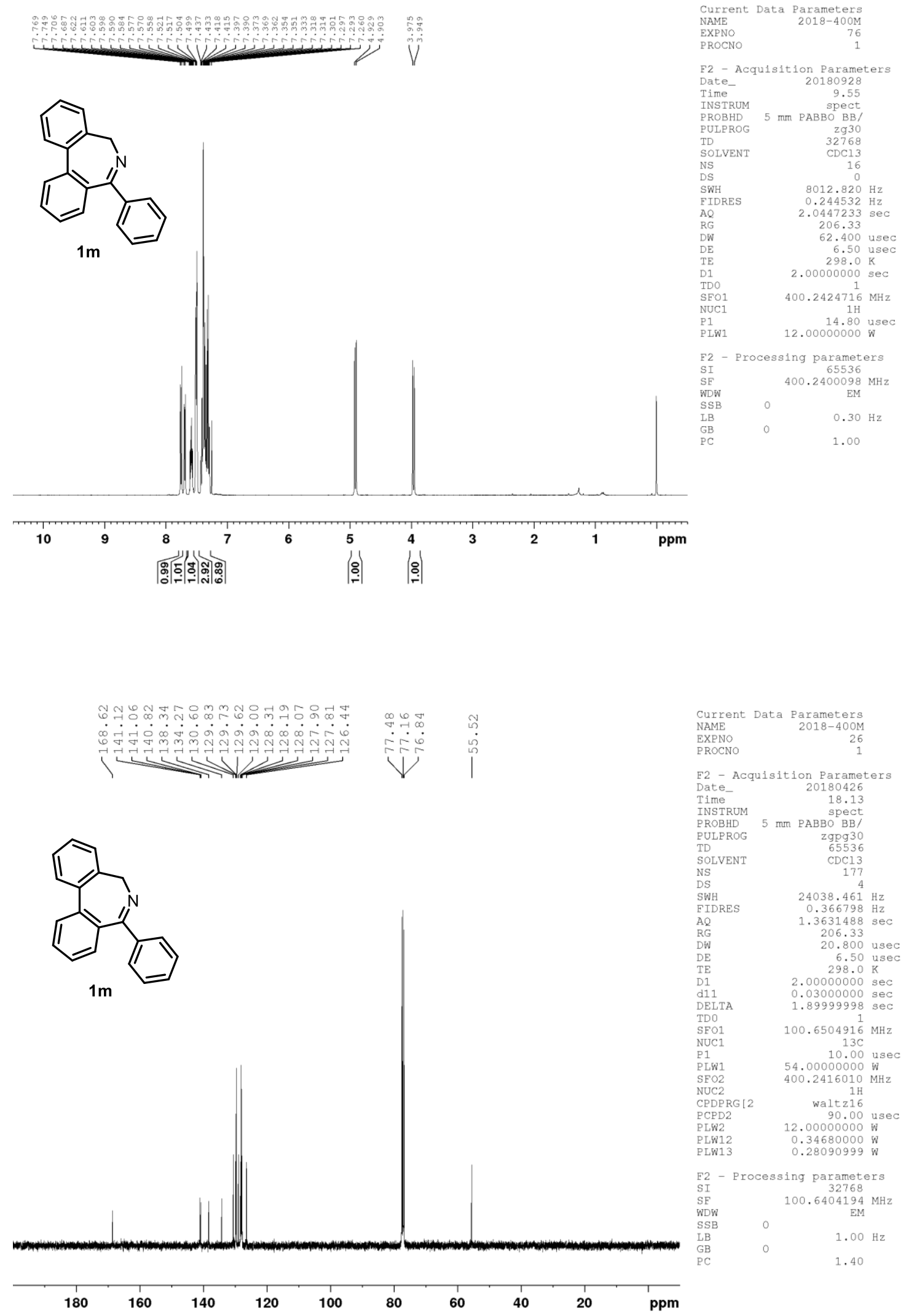


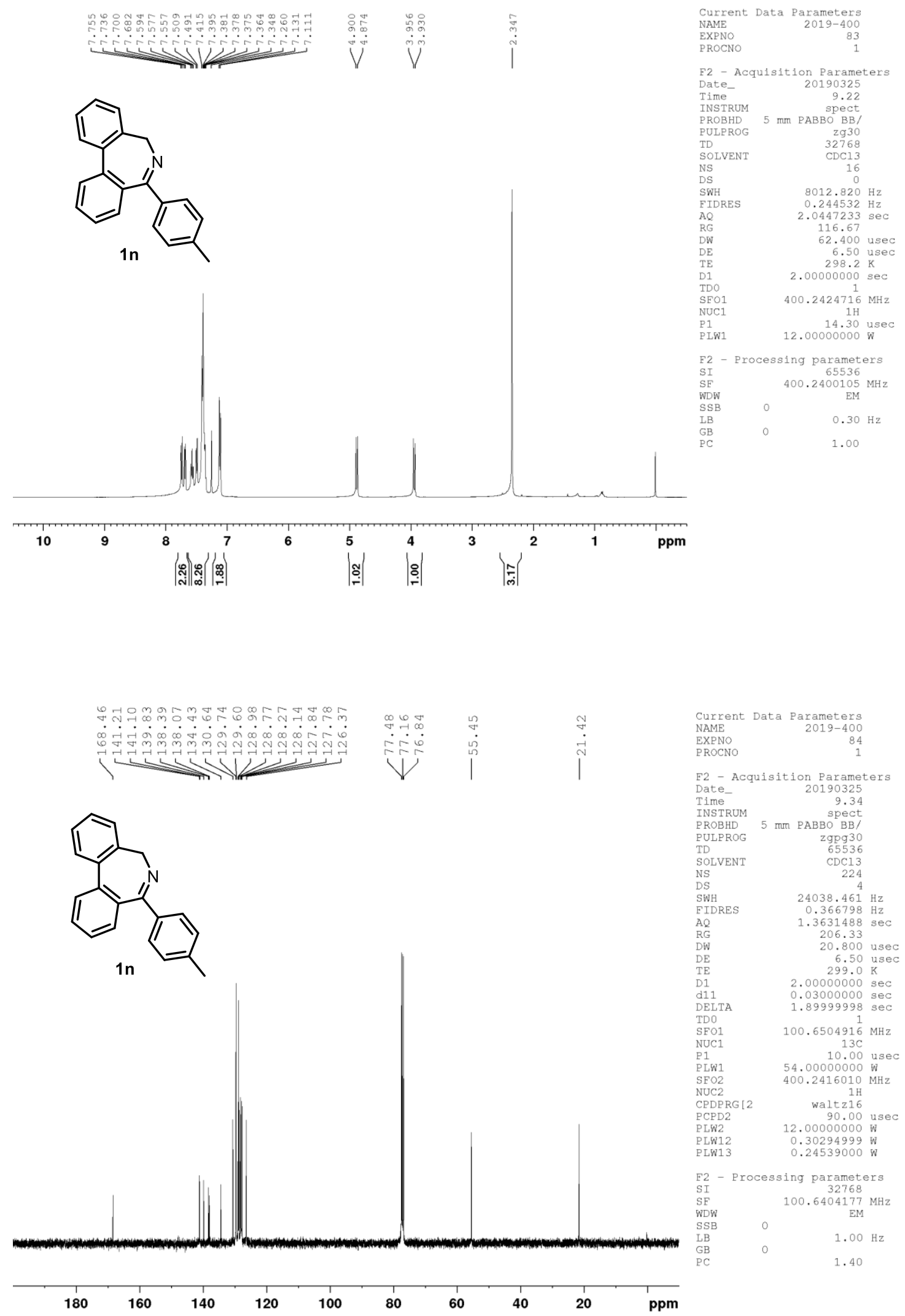



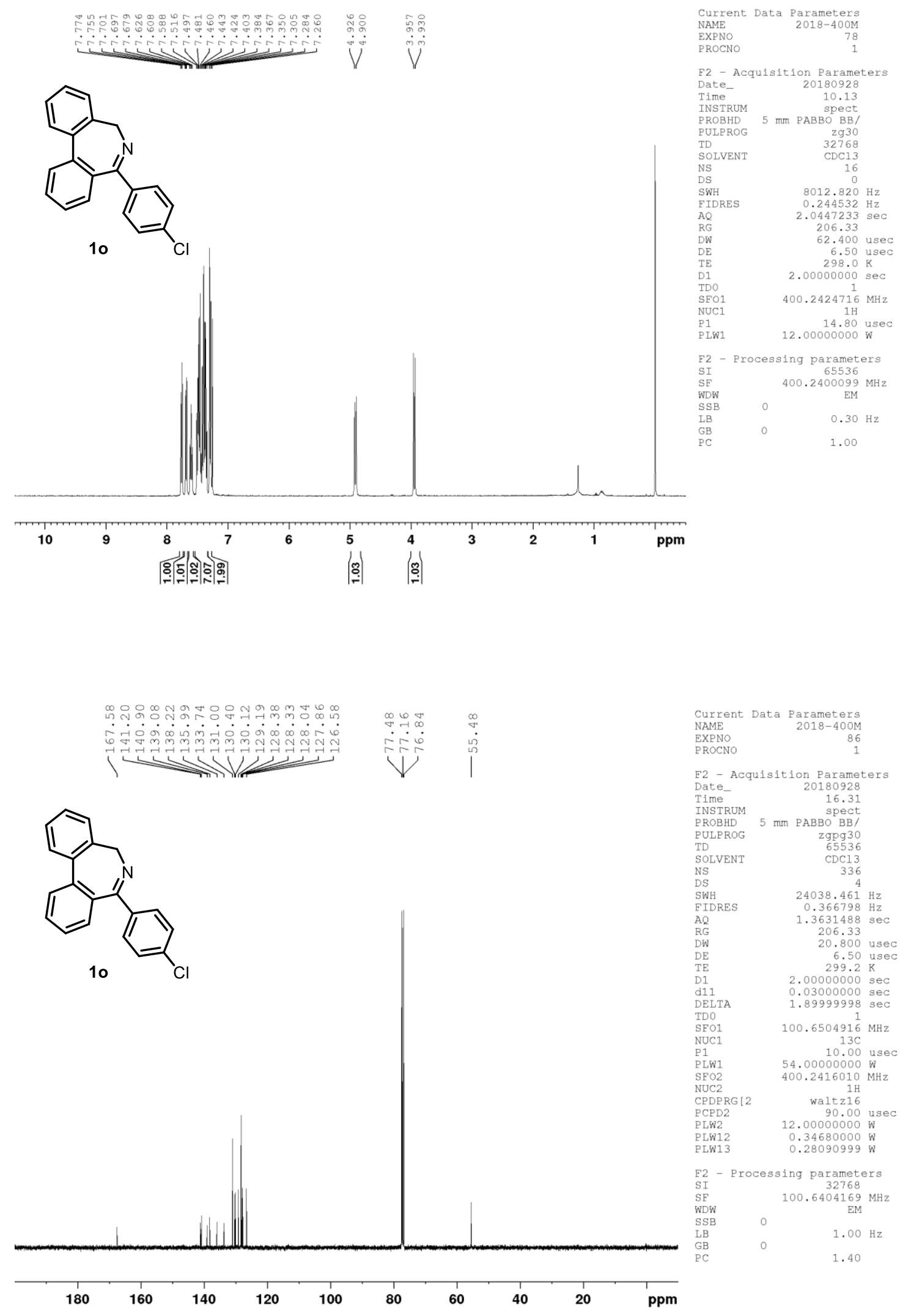
F2 - Acquisition Parameter

Date_ 20190516

Time $\quad 17.56$<smiles>Cc1ccc2c(c1)CN=C(c1ccccc1)c1ccccc1-2</smiles>

spect
PROBHD 5 mm PABBO BB

$\begin{array}{lr}\text { PULPROG } & \mathrm{zg} 30 \\ \text { TD } & 32768 \\ \text { SOLVENT } & \text { CDC13 }\end{array}$

$\begin{array}{lr}\text { DS } & 0 \\ \text { SWH } & 8012.820 \mathrm{~Hz}\end{array}$

AQ $\quad 2.0447233 \mathrm{sec}$

$\begin{array}{lr}\text { RG } & 65.71 \\ \text { DW } & 62.400 \text { use }\end{array}$

$\begin{array}{lr}\text { DE } & 6.50 \text { use } \\ \text { TE } & 298.6 \mathrm{~K}\end{array}$

$\begin{array}{lr}298.6 \mathrm{~K} \\ \mathrm{D} 1 & 2.00000000 \mathrm{sec}\end{array}$

TDO1 $400.2424716 \mathrm{MH}$

$\begin{array}{lr}\text { NUC1 } & 400.242416 \\ \text { P1 } & 14.30\end{array}$

P1 $\quad 12.00000000 \mathrm{~W}$

F2 - Processing parameters

$\begin{array}{lr}\text { SI } & 400.24001962 \mathrm{MHz} \\ \text { SF } & \end{array}$

WDW $\quad \begin{aligned} & 400.2400192 \\ & \text { EM }\end{aligned}$

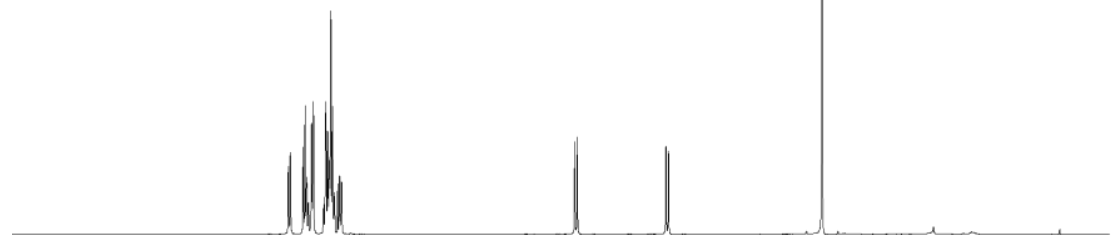

$\begin{array}{lll}\mathrm{LB} & 0.30 \mathrm{~Hz} \\ \mathrm{CB} & 0 & 1.00\end{array}$
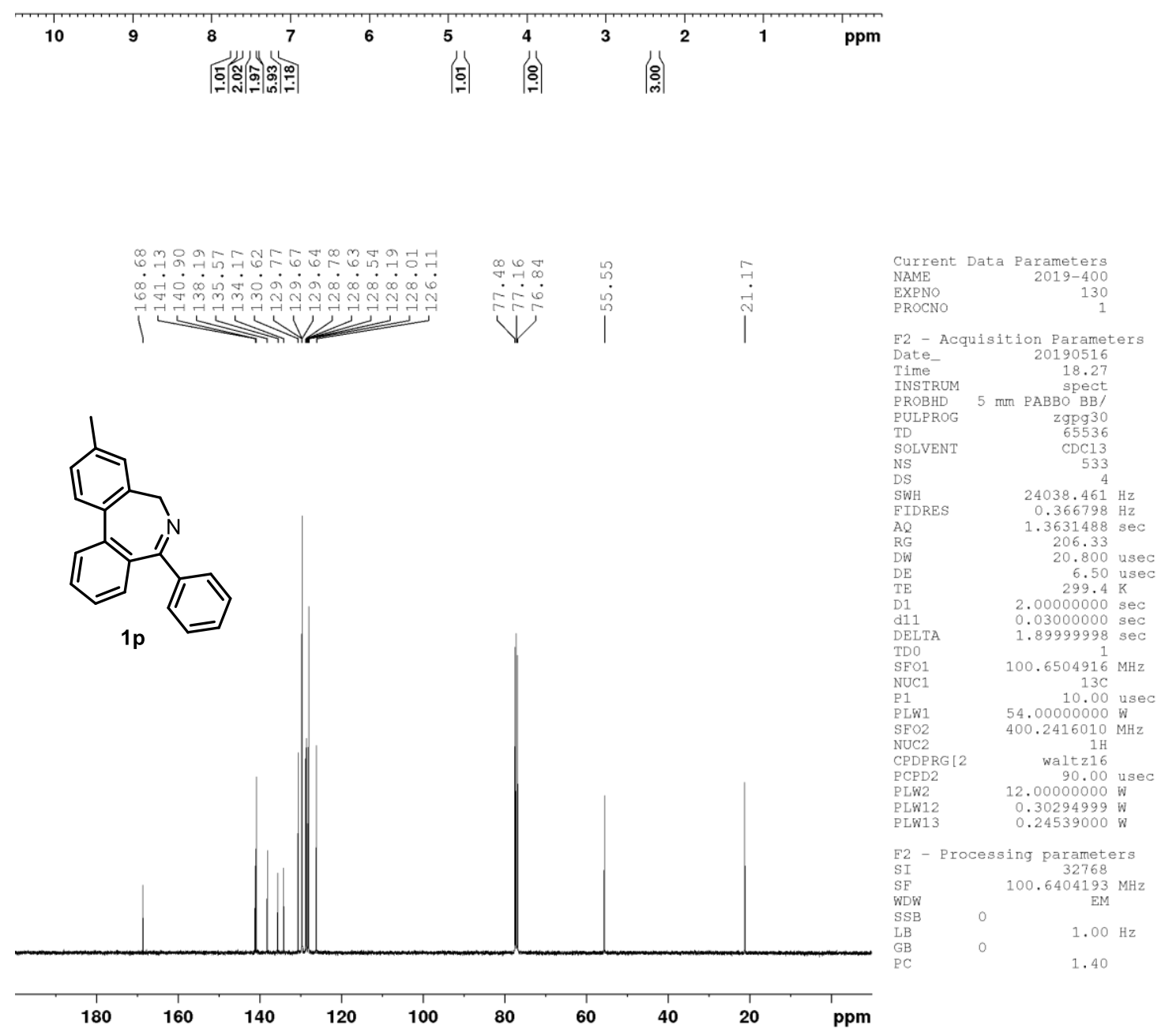

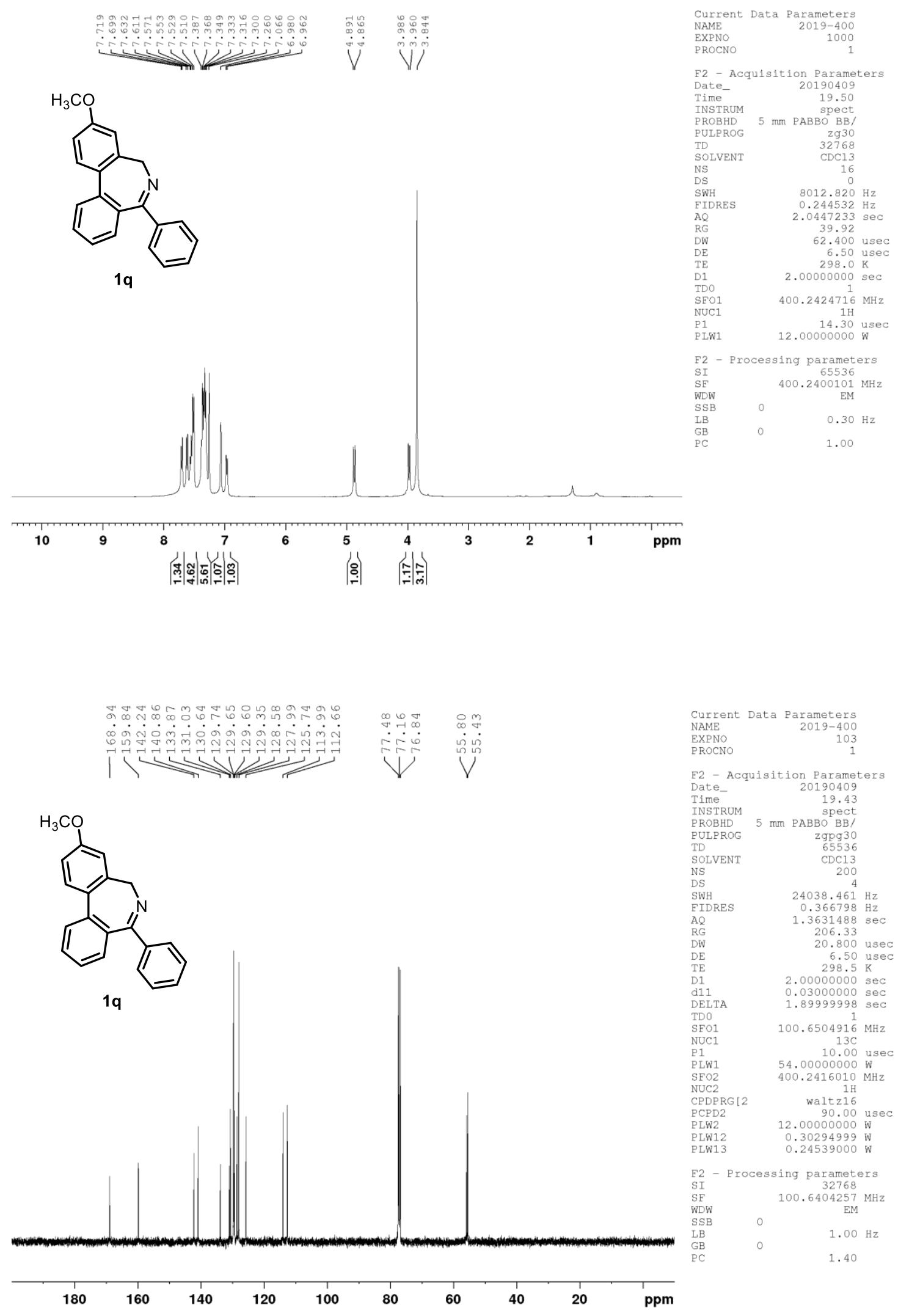

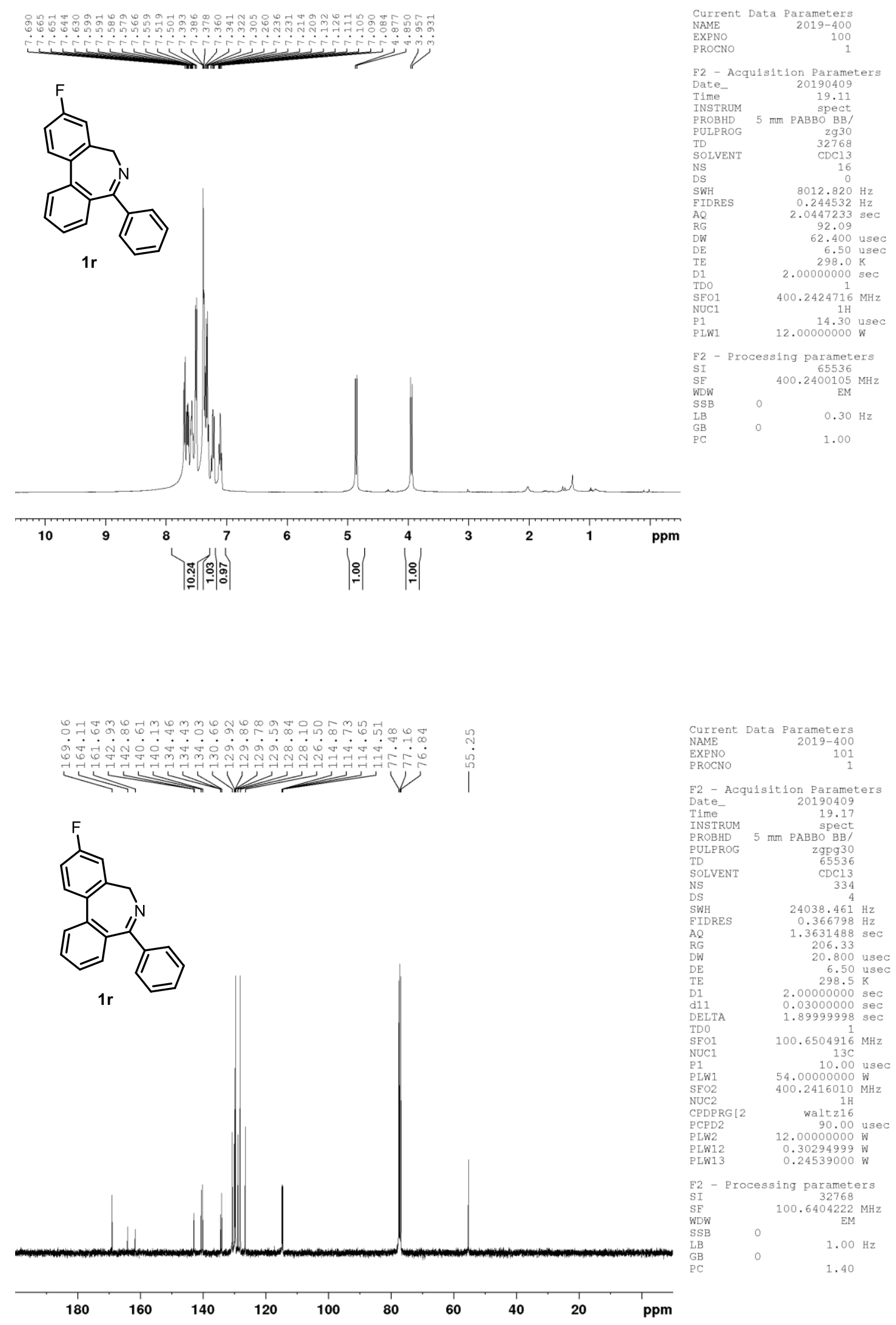


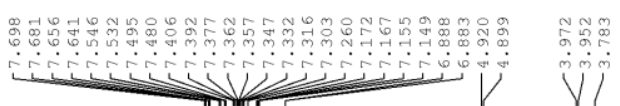<smiles></smiles>

$1 \mathrm{~s}$
Current Data Parameters
NAME
2019-500

EXPNO

F2 - Acquisition Parameter

Date__ 20190507

TNSTRUM

PROBHD $5 \mathrm{~mm}$ CPPBBO BB

PULPROG 5

$\begin{array}{lr}\text { TD } & 65536 \\ \text { SOLVENT } & \text { CDC13 }\end{array}$

SOLVENT

$\begin{array}{lr}\text { DS } & 2 \\ \text { SWH } & 10000.000 \mathrm{~Hz}\end{array}$

AQ $\quad 3.2767999 \mathrm{sec}$

$\begin{array}{lr}R G & 31.72 \\ \text { DW } & 50.000 \text { usec } \\ \text { DE } & 6.50 \text { usec }\end{array}$

TE $\quad 298.1 \mathrm{~K}$

TDO $\quad 1.00000000 \mathrm{sec}$

SEO1
NUC1

$\begin{array}{ll}\text { P1 } & 11.50 \text { usec }\end{array}$

F2 - Processing parameters

$\begin{array}{lr}\text { F2 } & 65536 \\ \mathrm{SI} & 500.1300122 \mathrm{MHz}\end{array}$

$\begin{array}{lr}\text { SE } & 500.1300122 \\ \text { WDW } & \text { EM }\end{array}$

$\begin{array}{lll}\mathrm{LB} & 0.30 \mathrm{~Hz}\end{array}$

GB
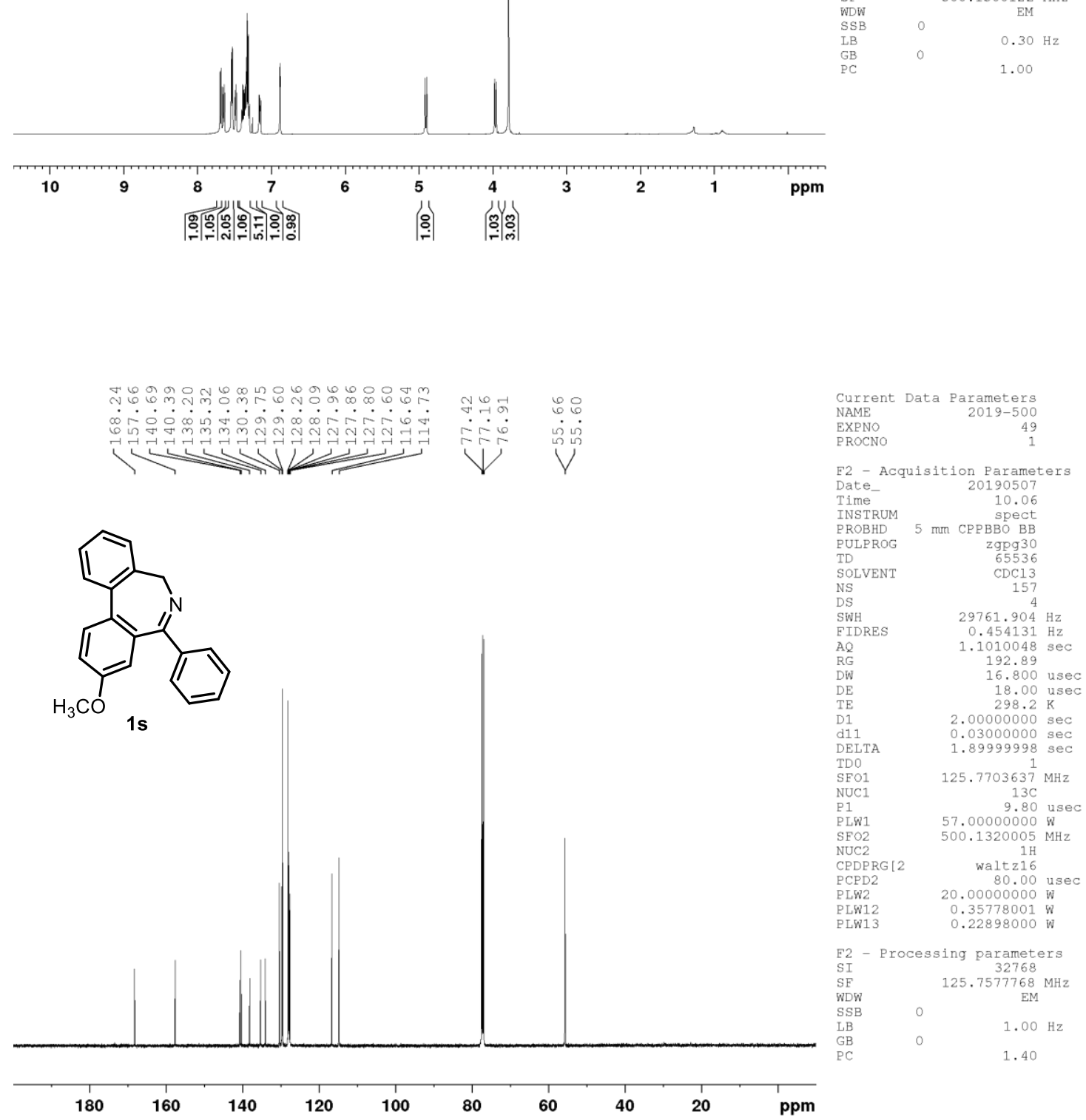

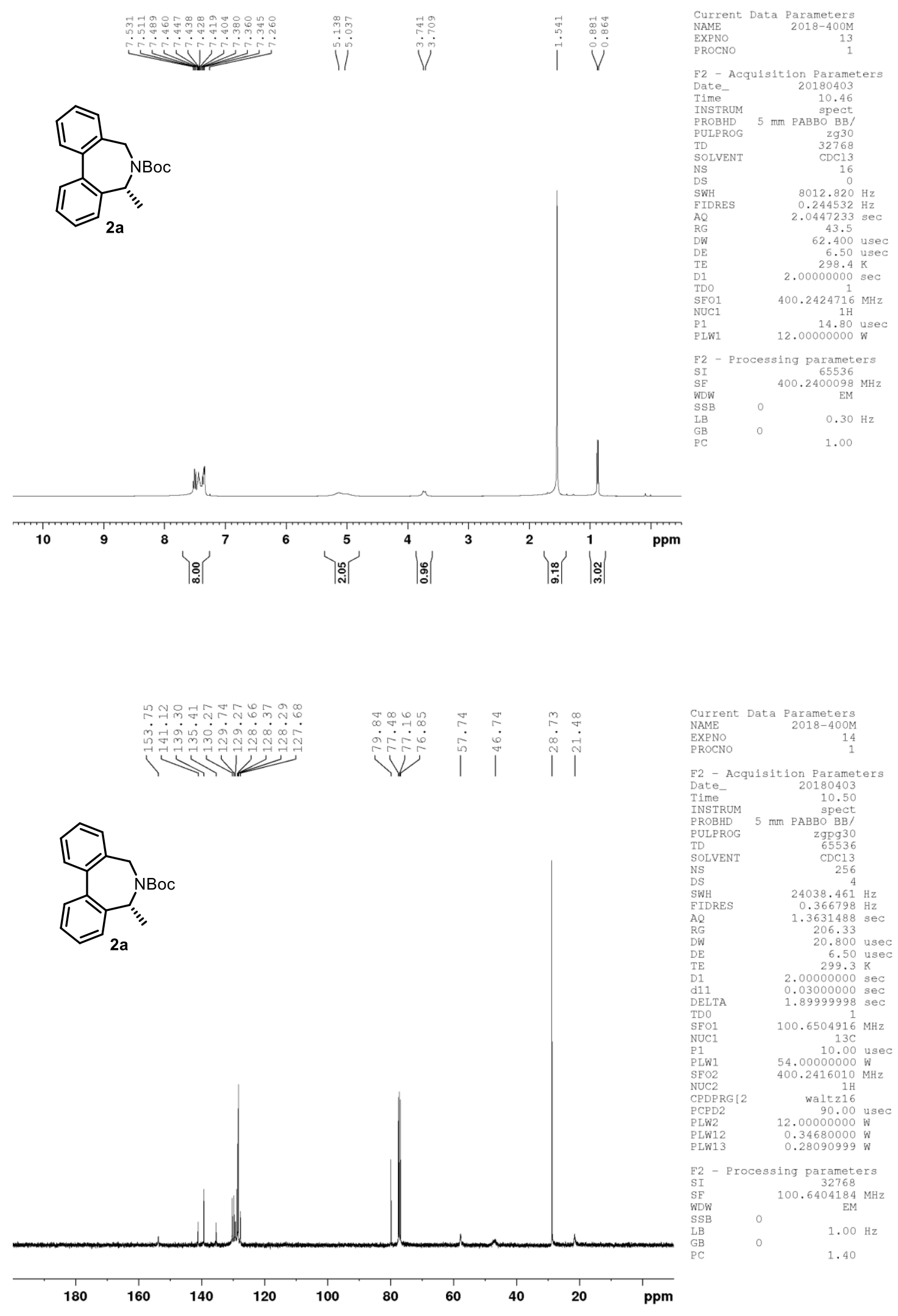


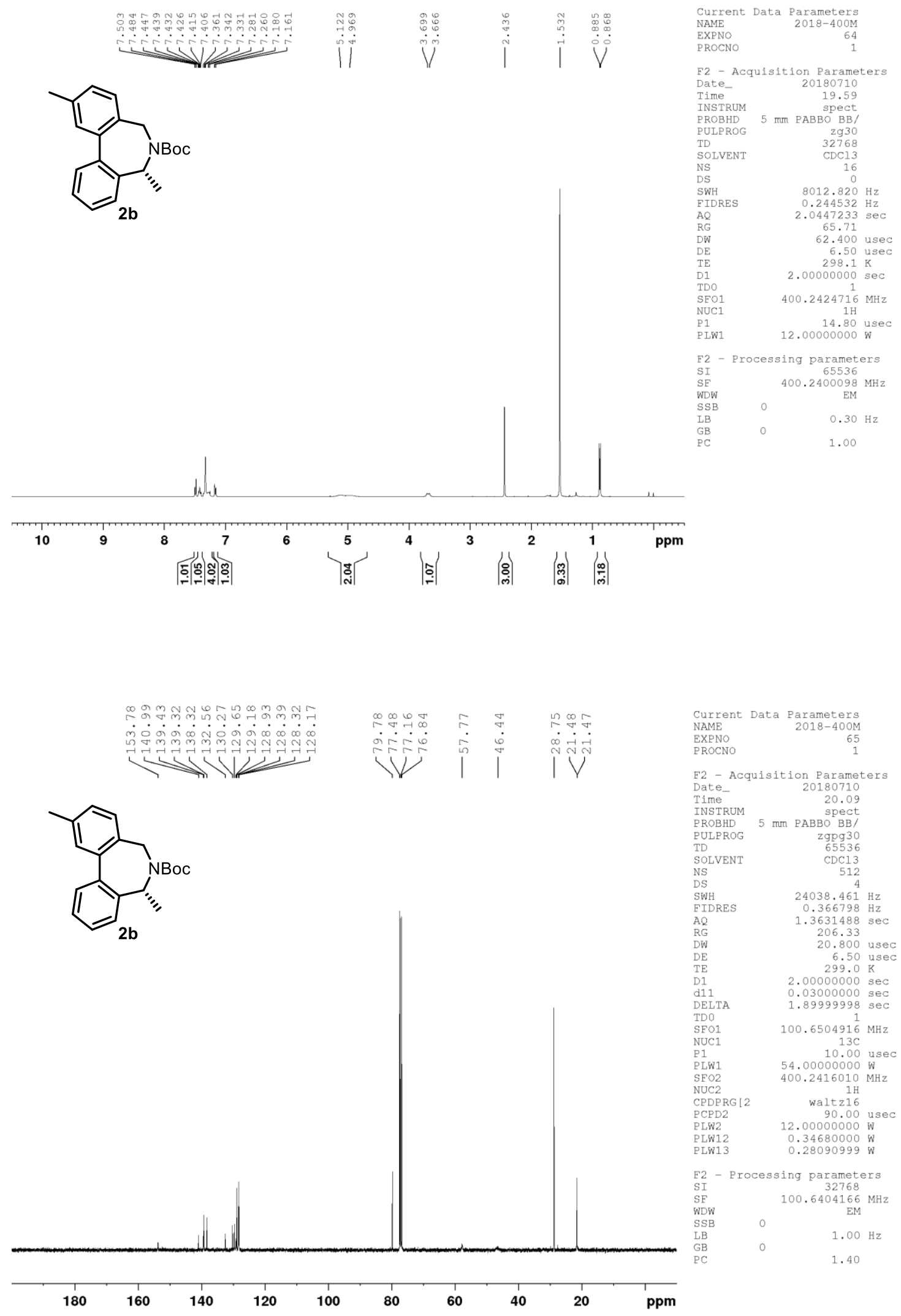



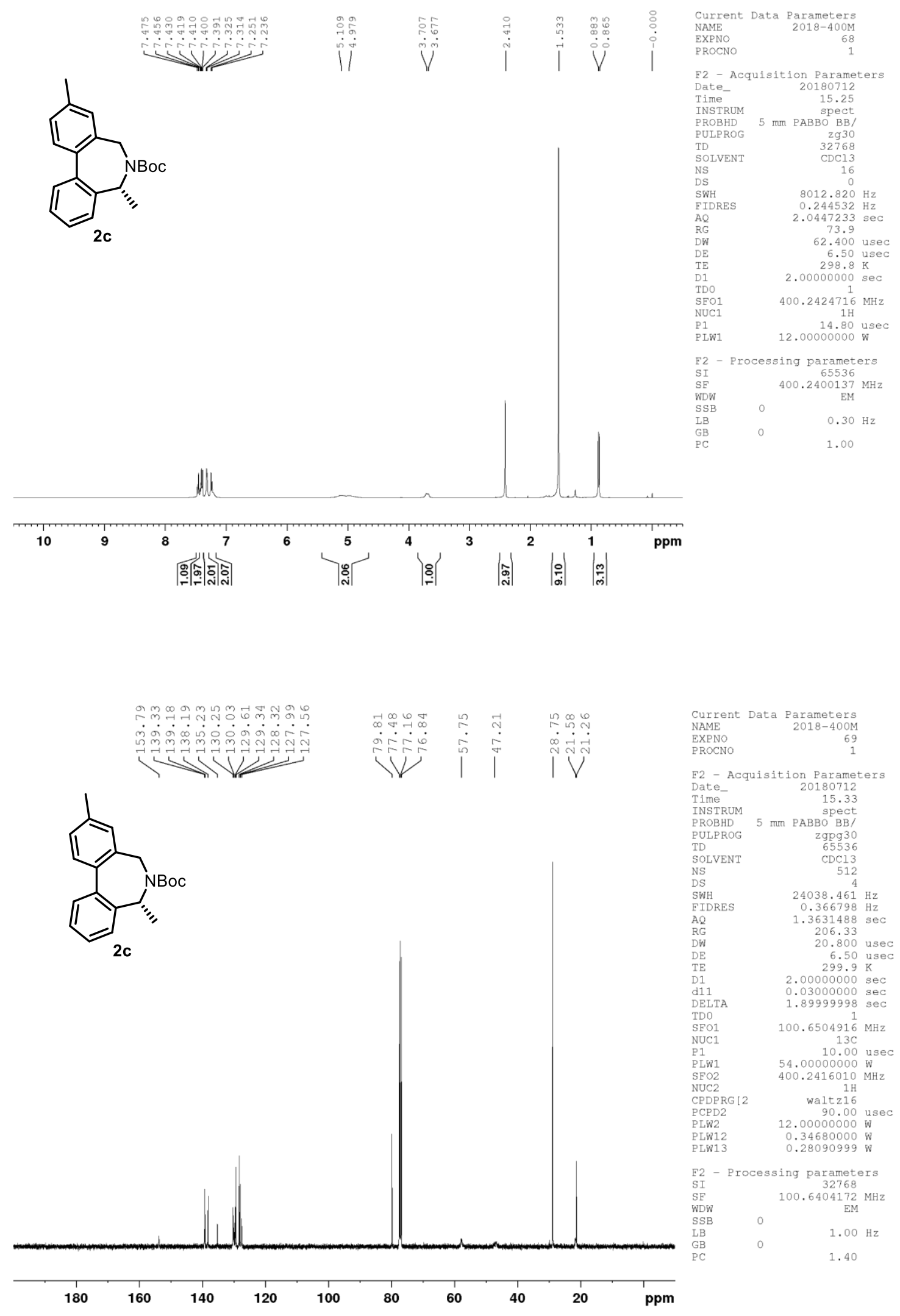

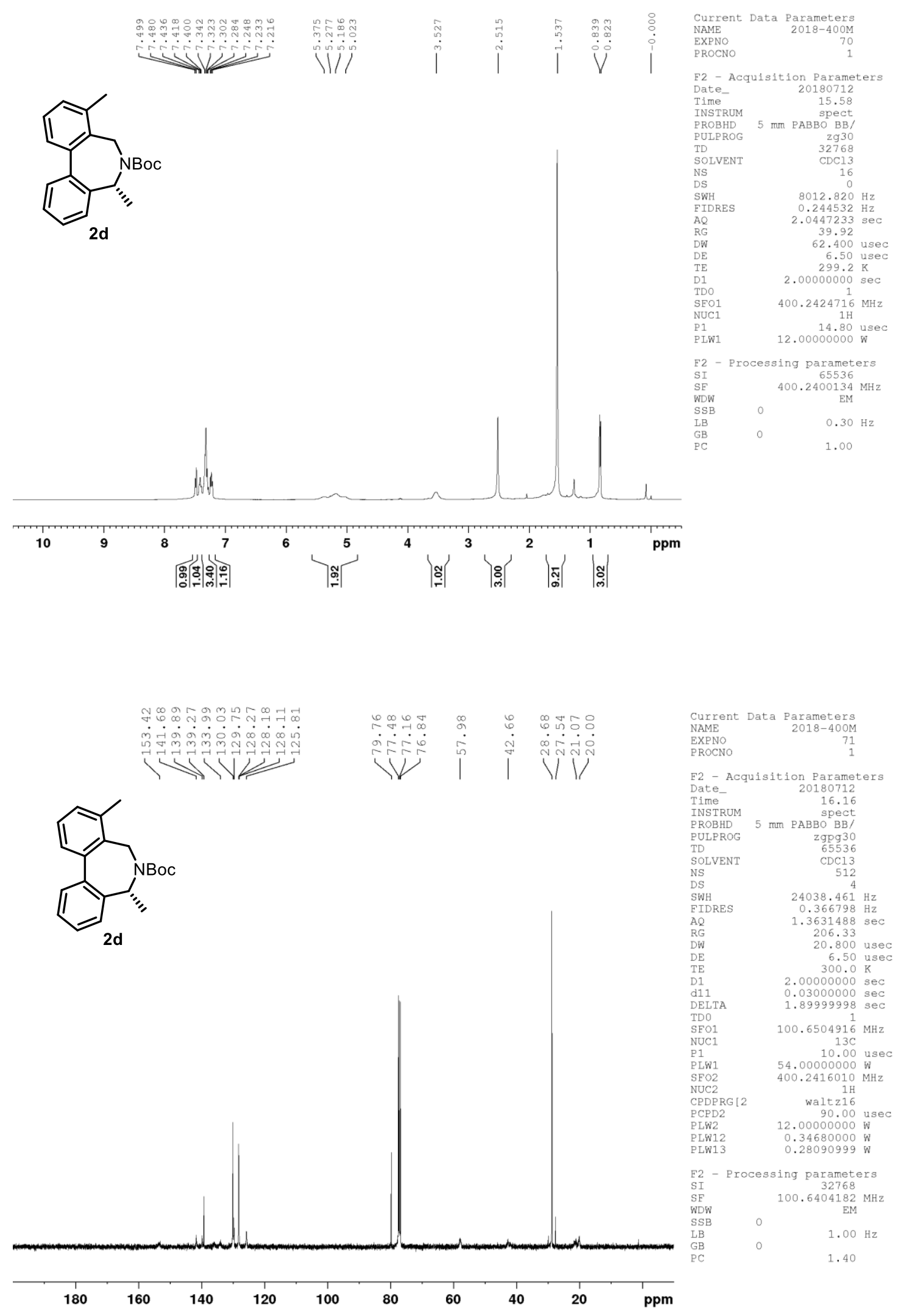


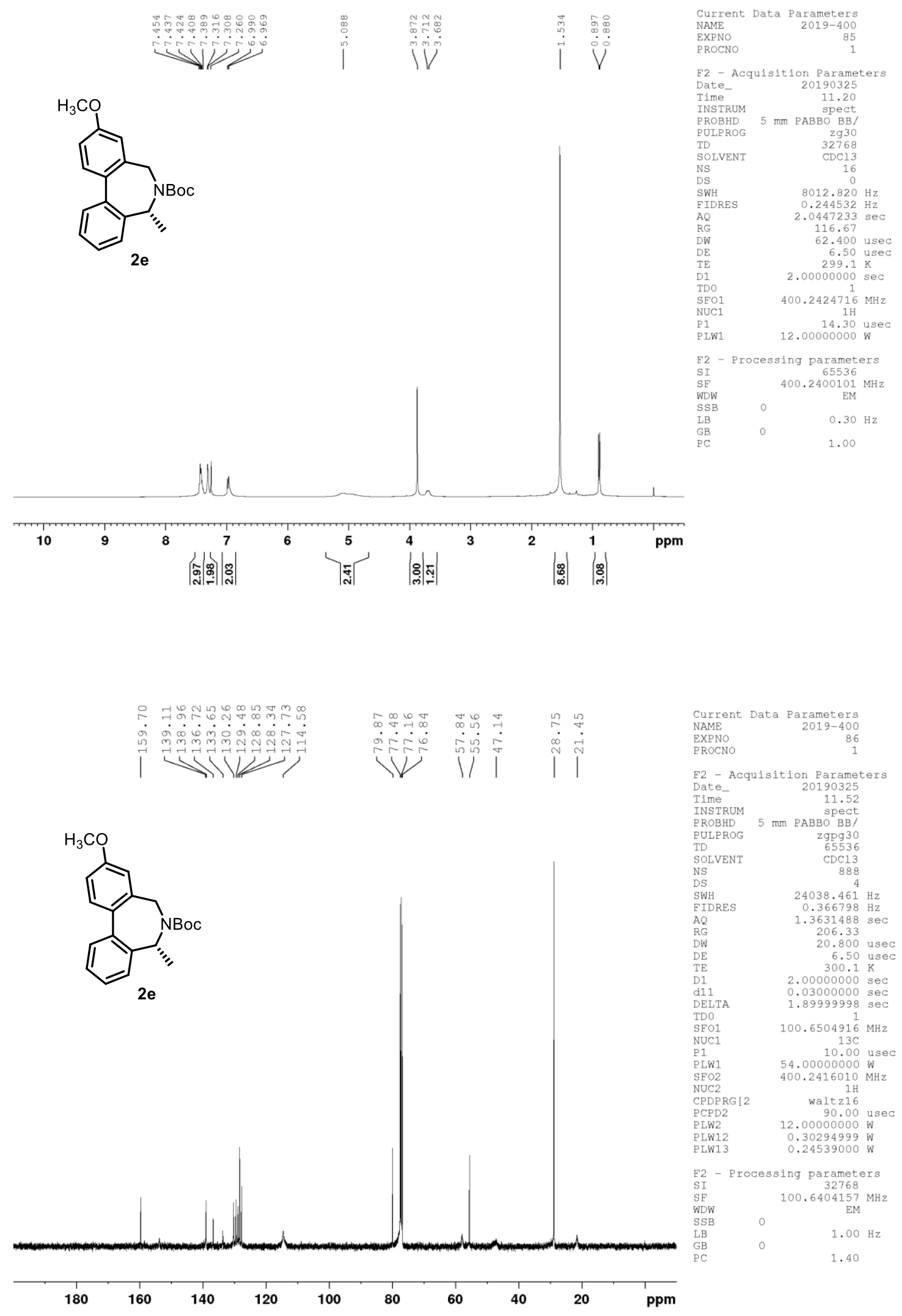



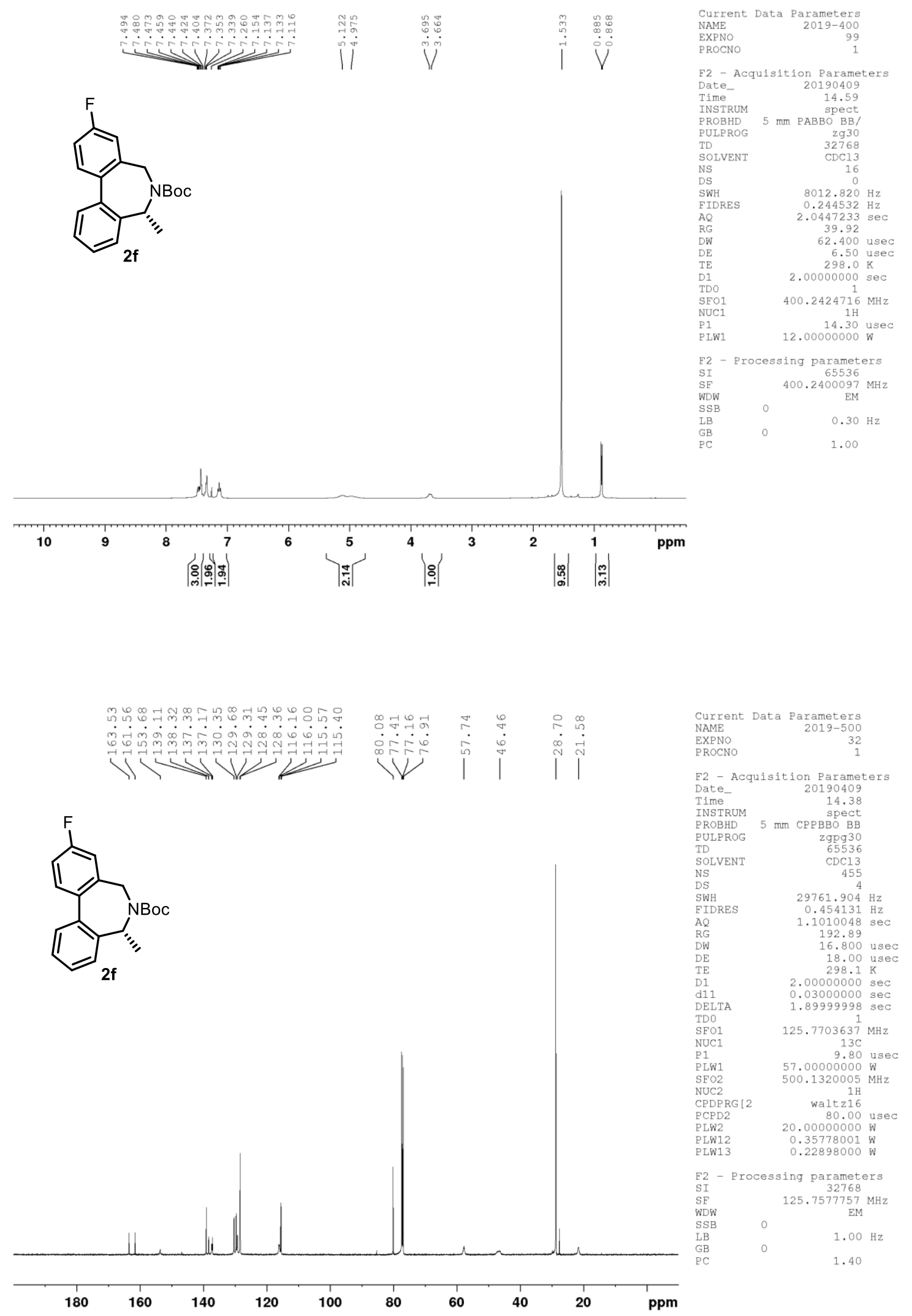


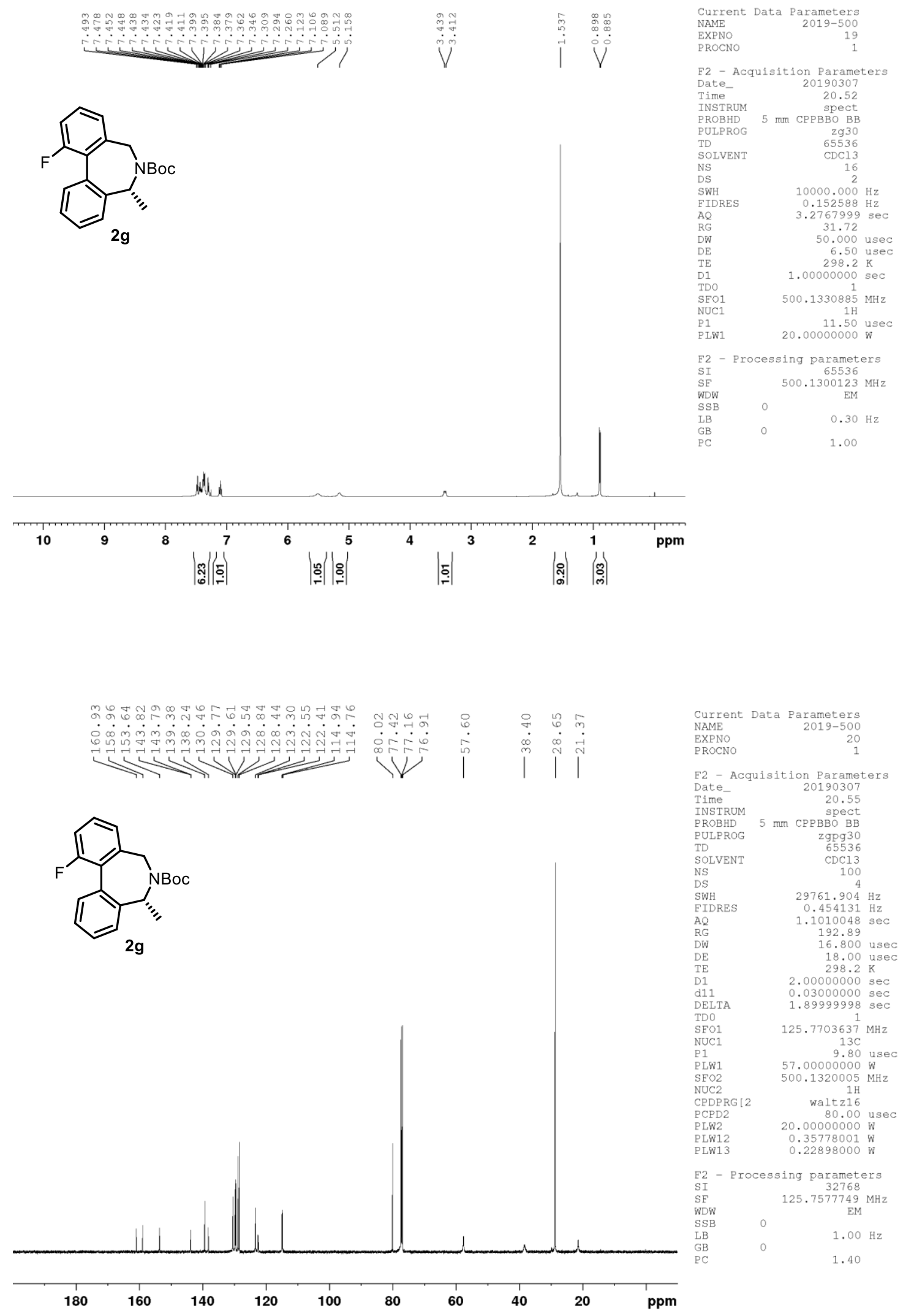




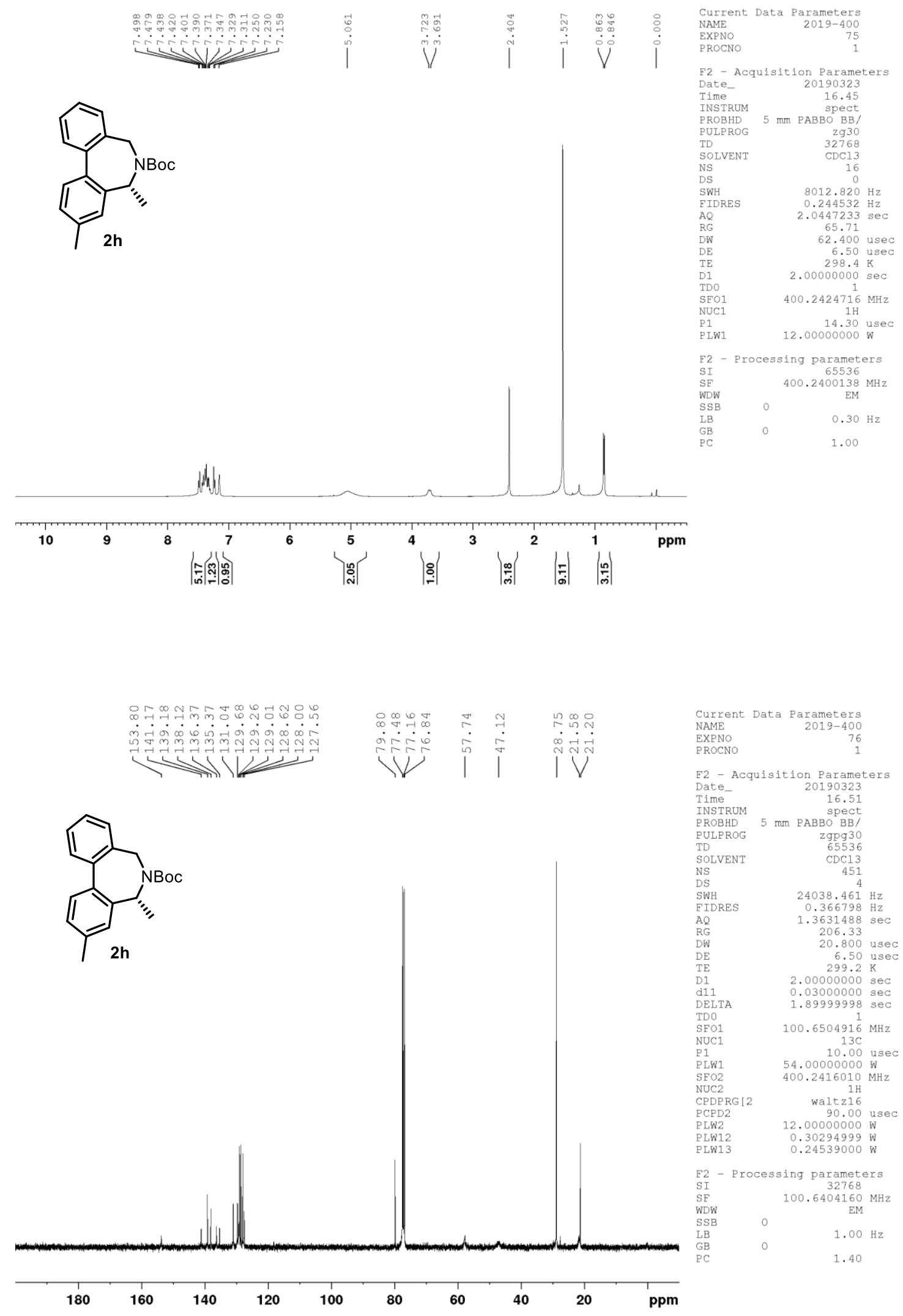




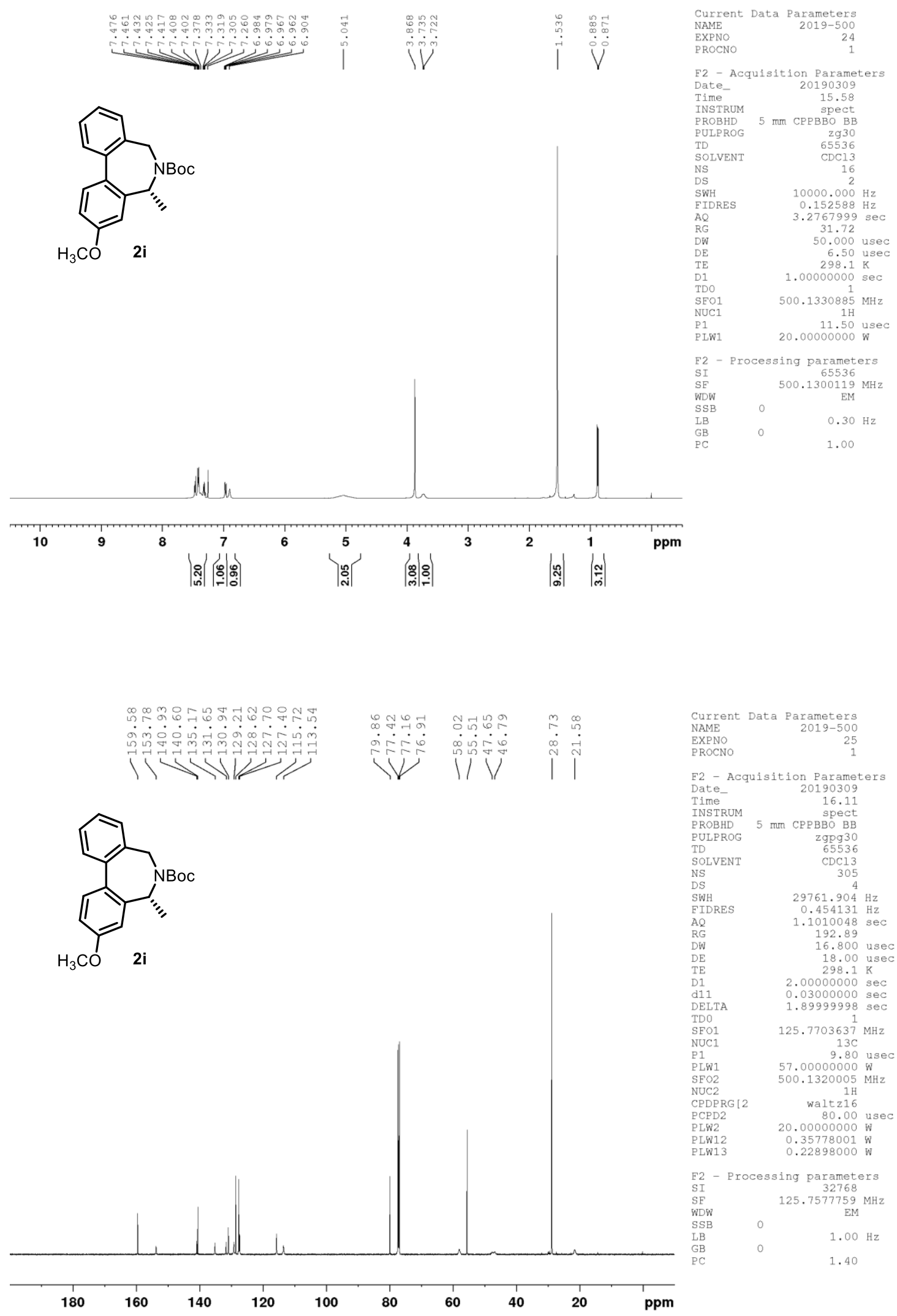




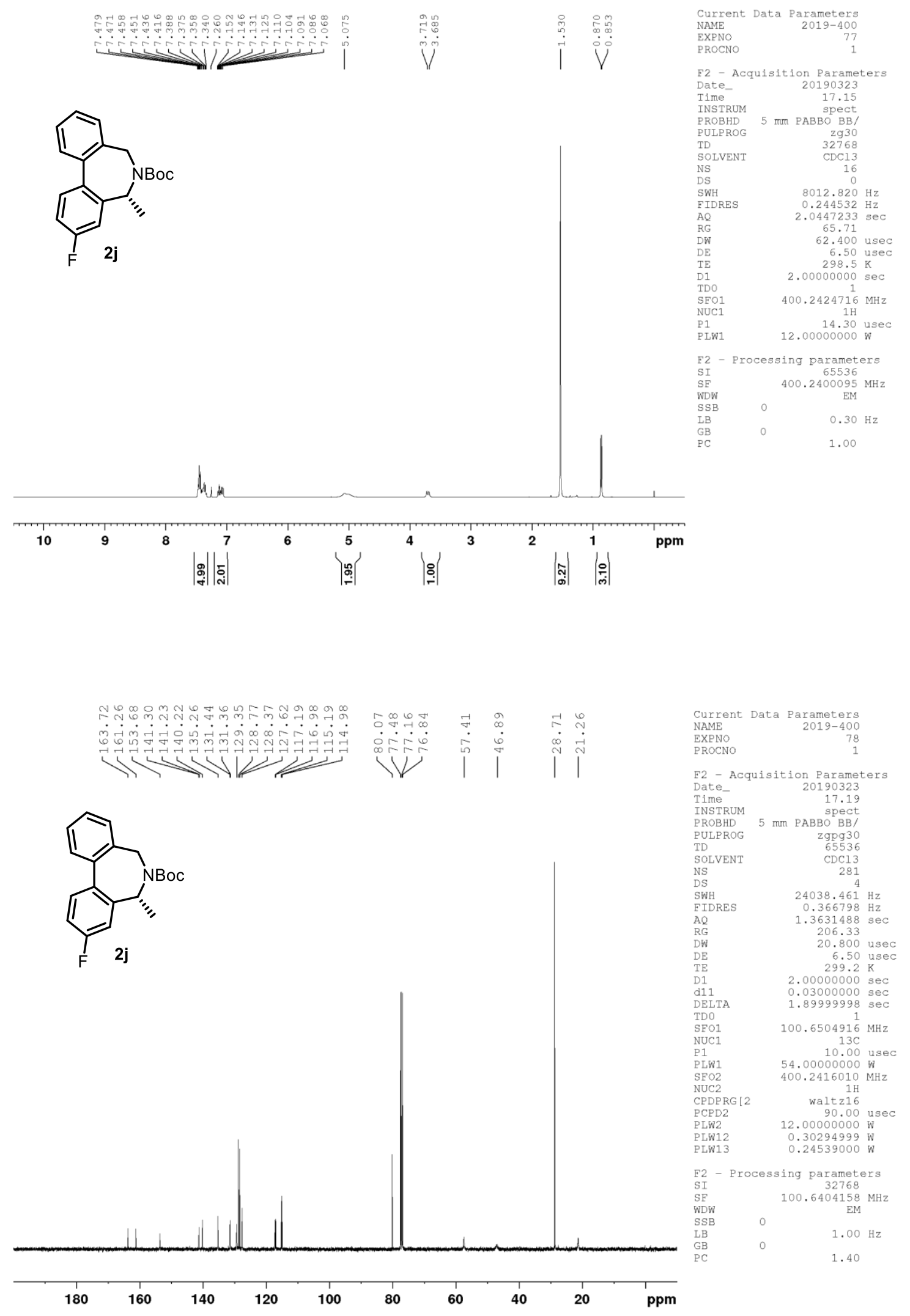



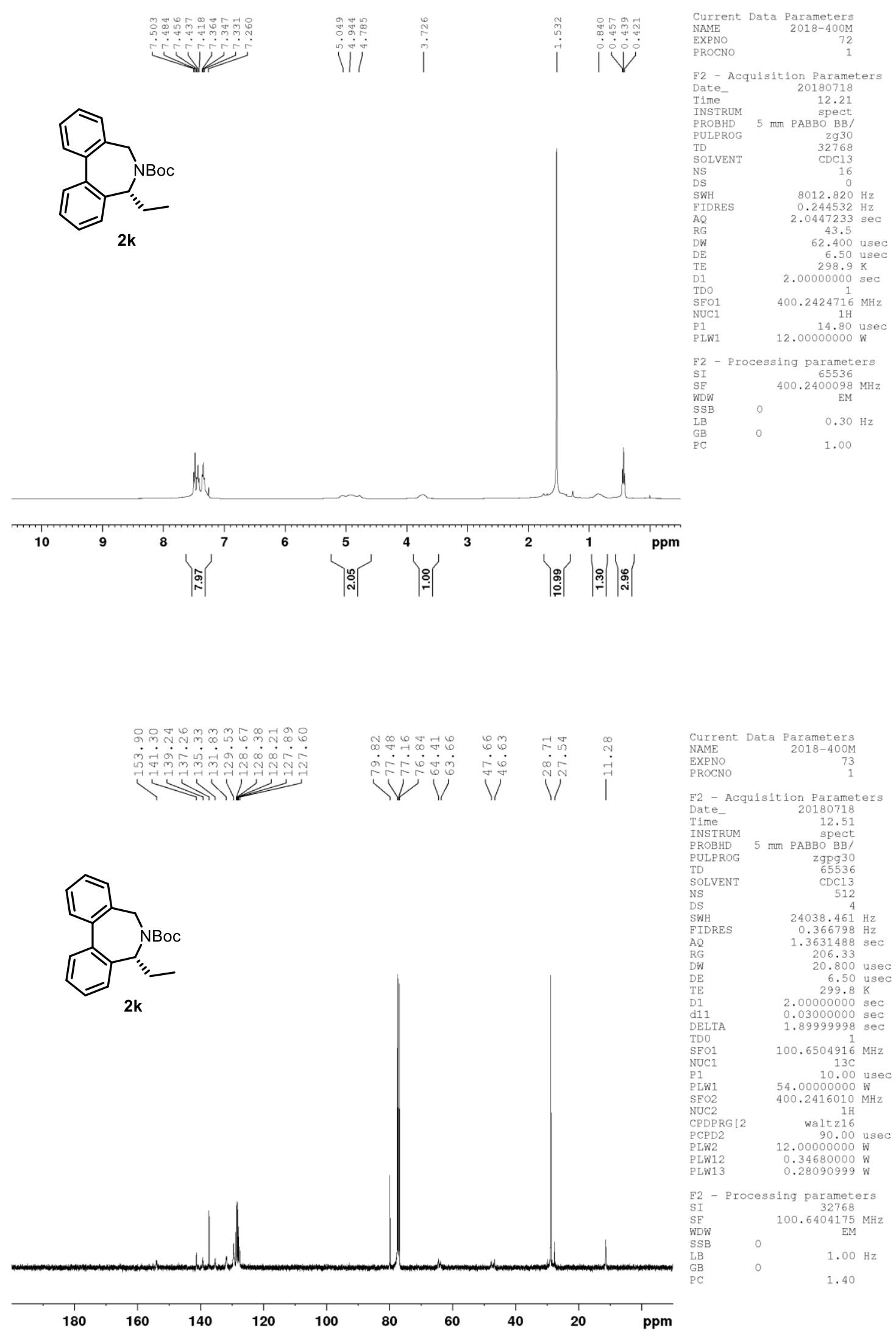


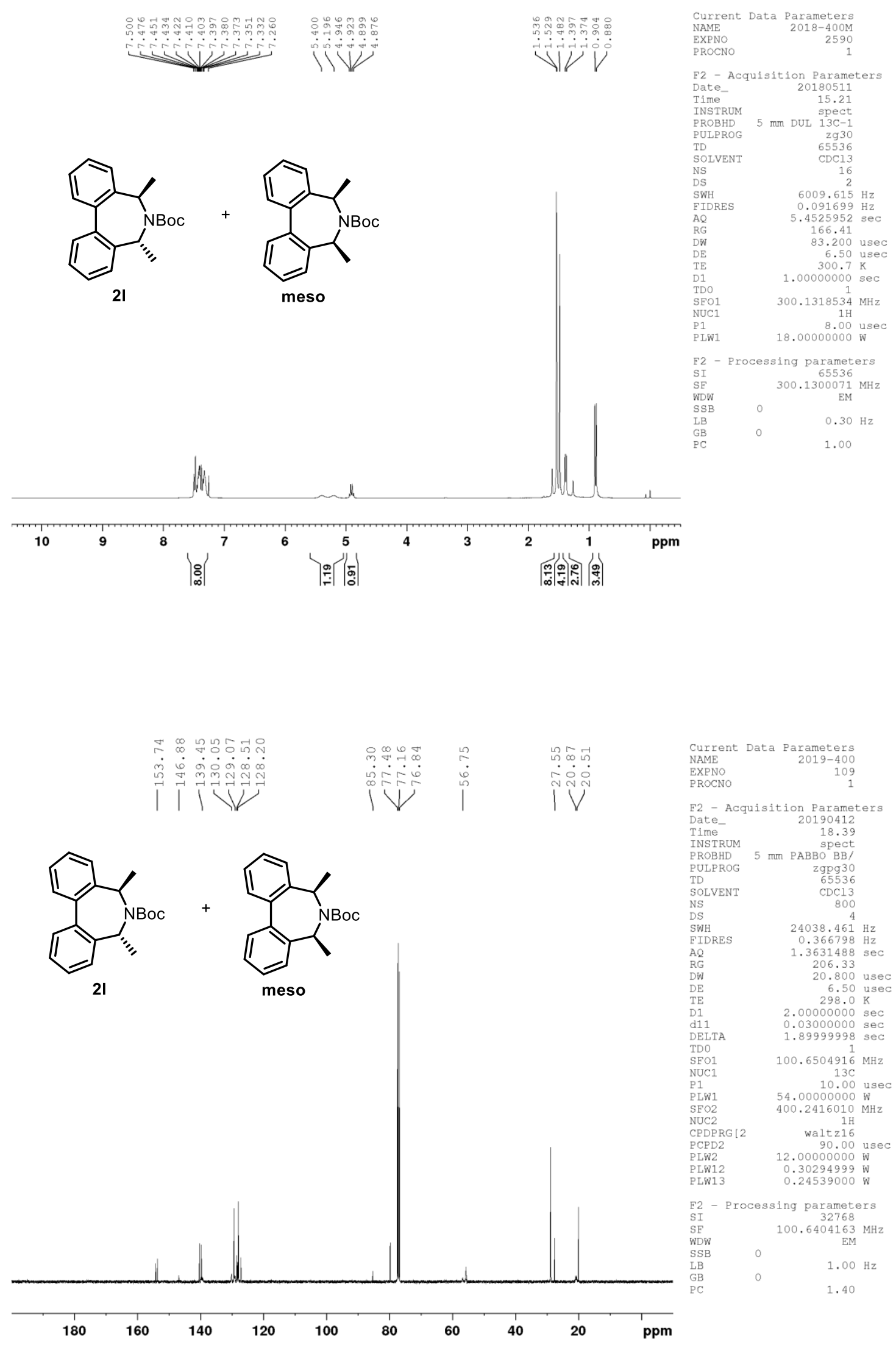



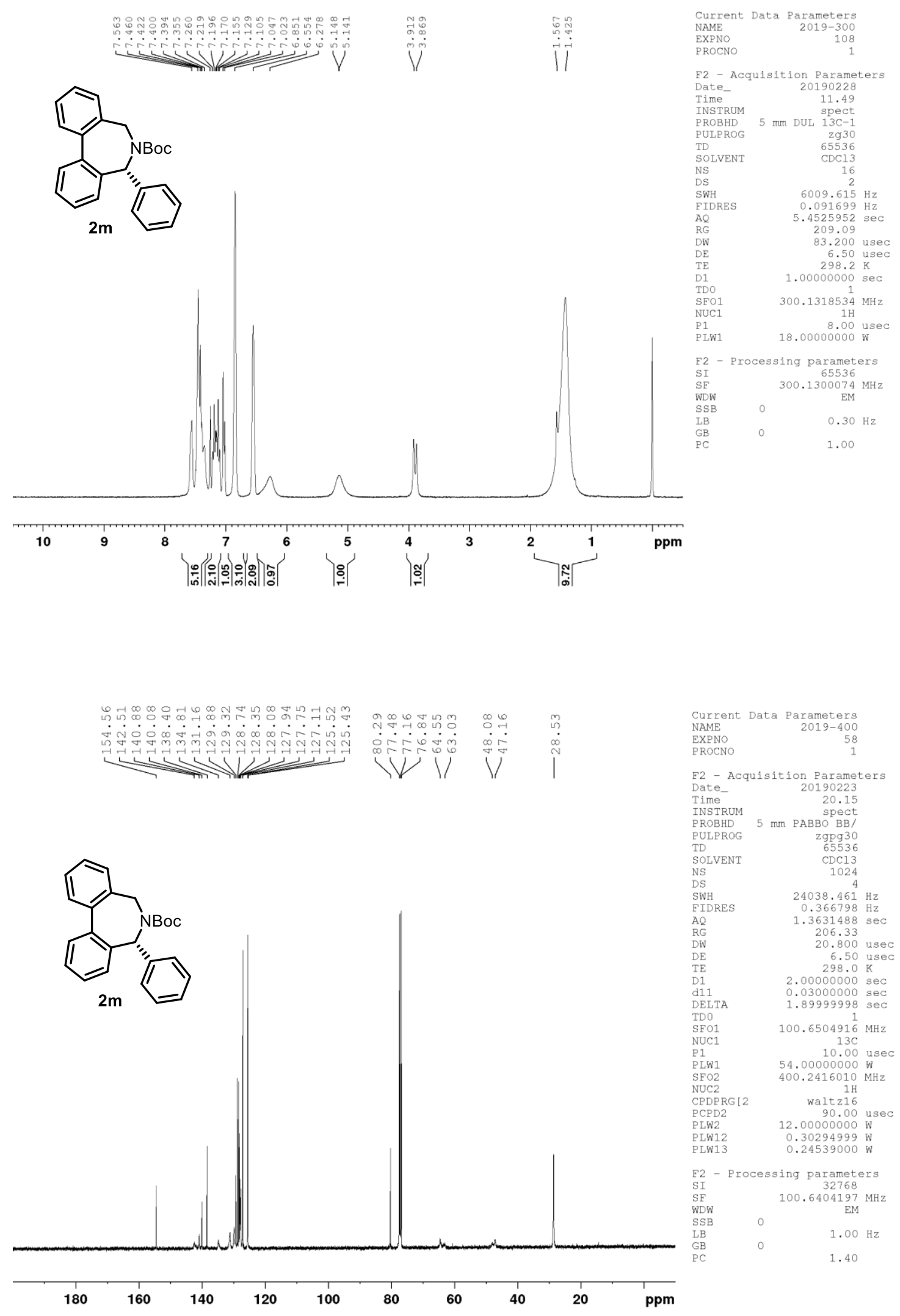


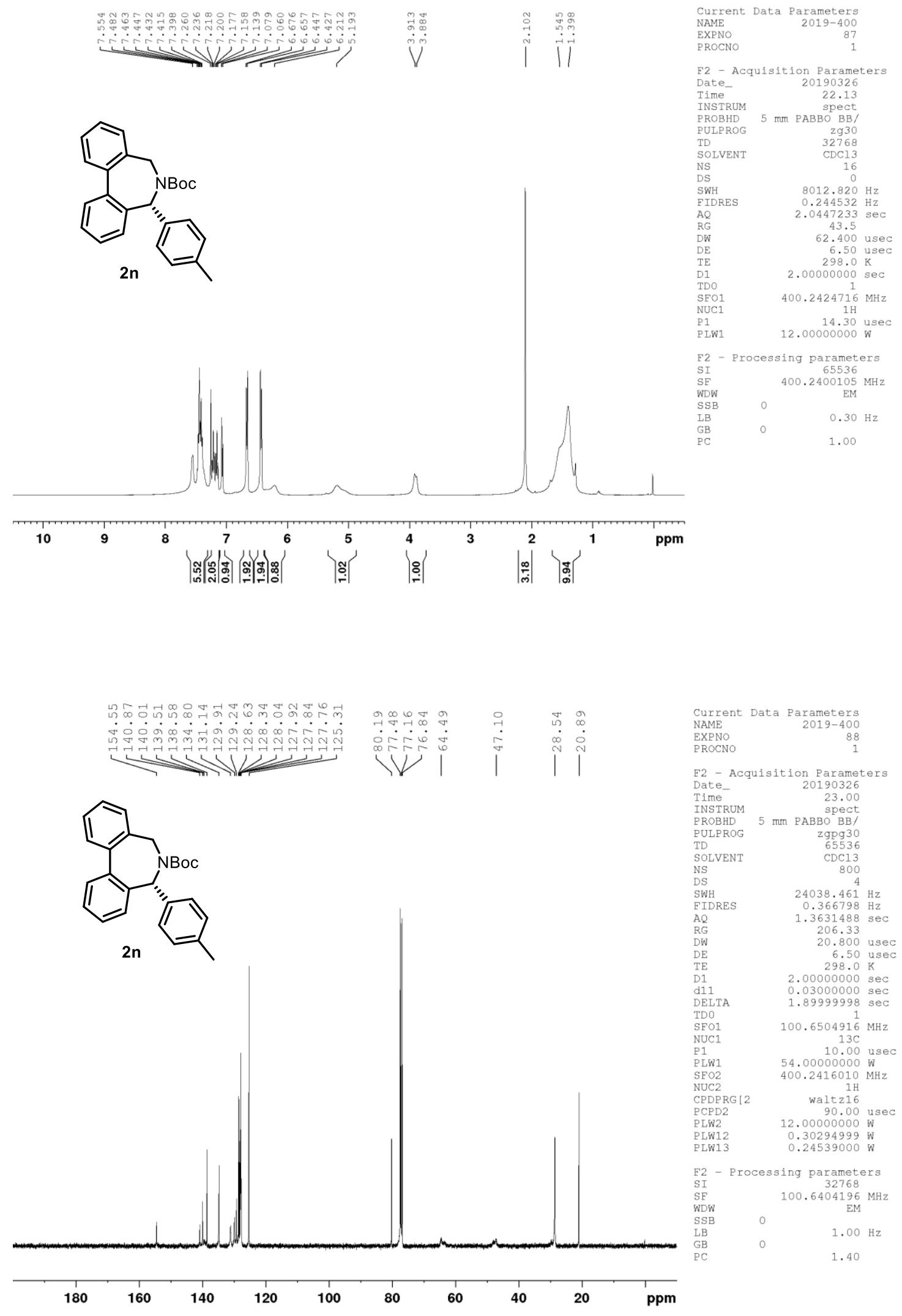



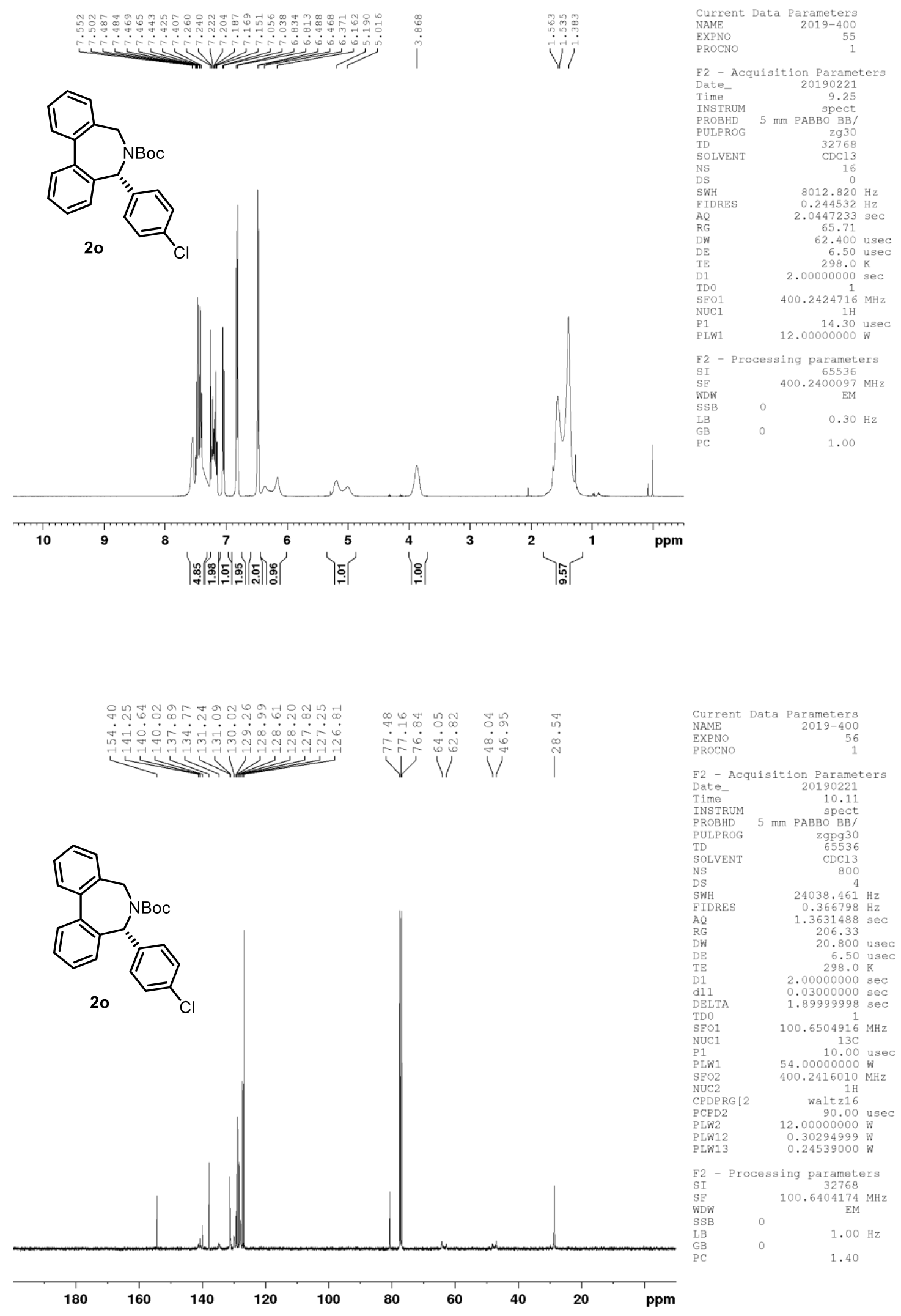


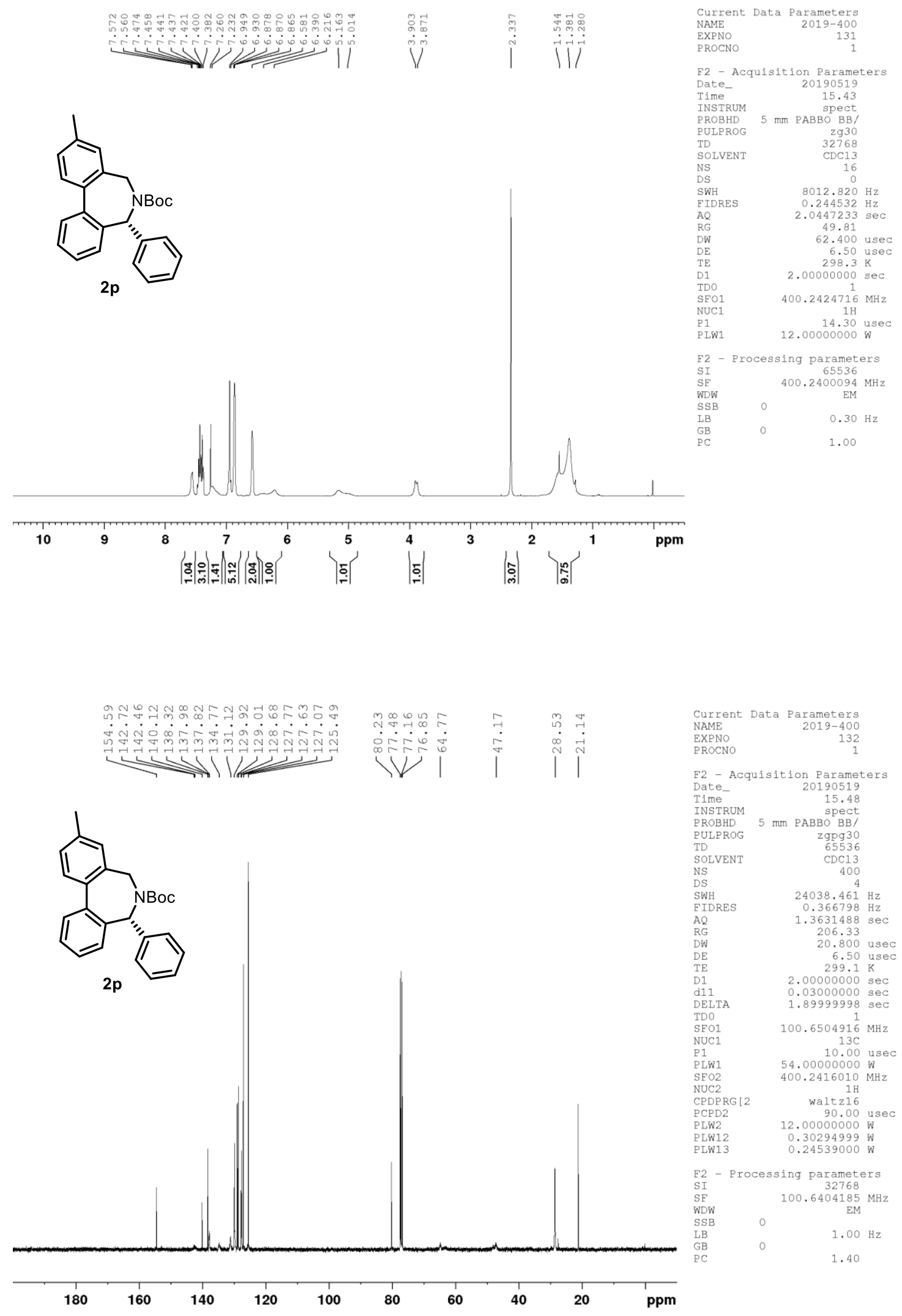




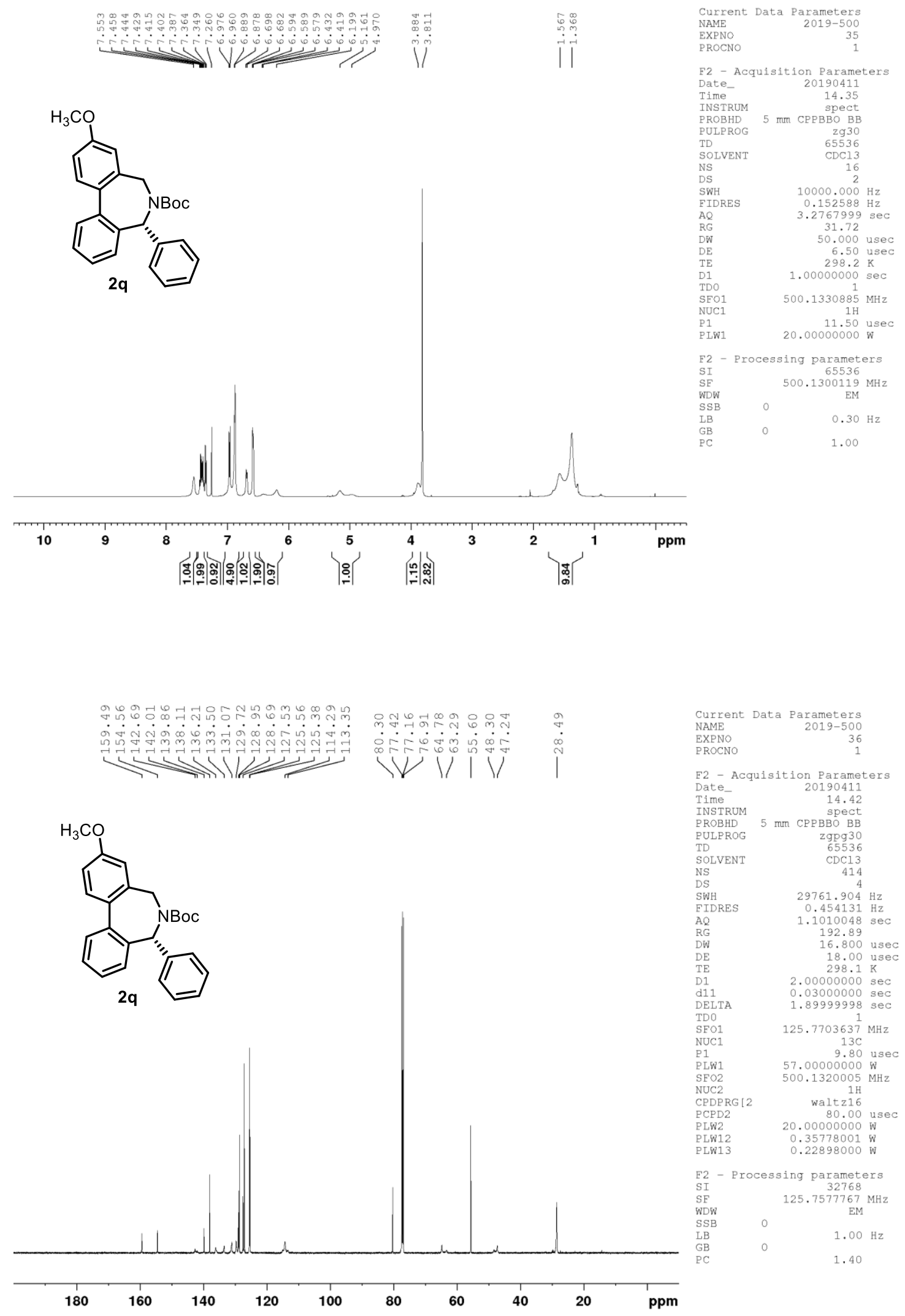




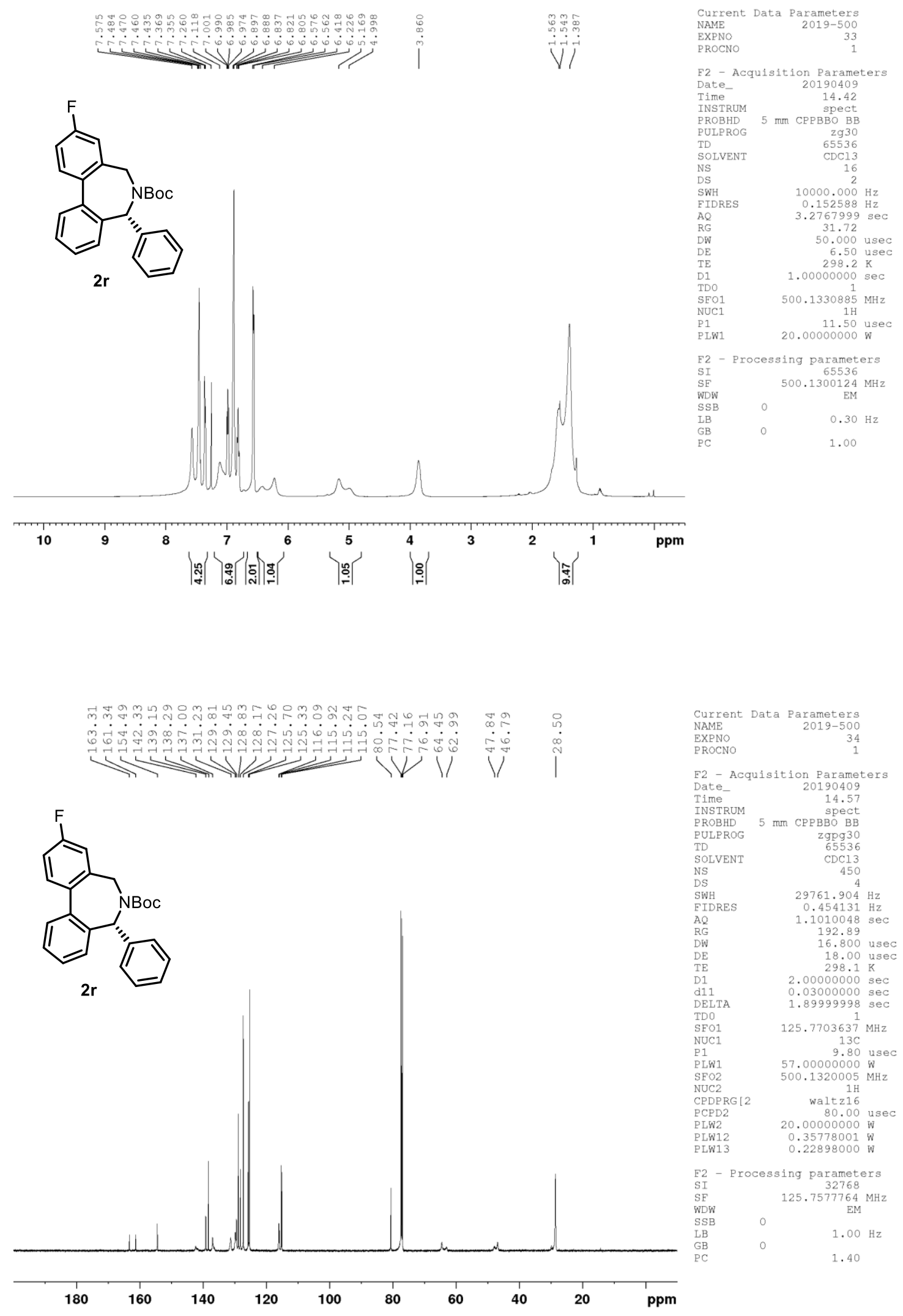




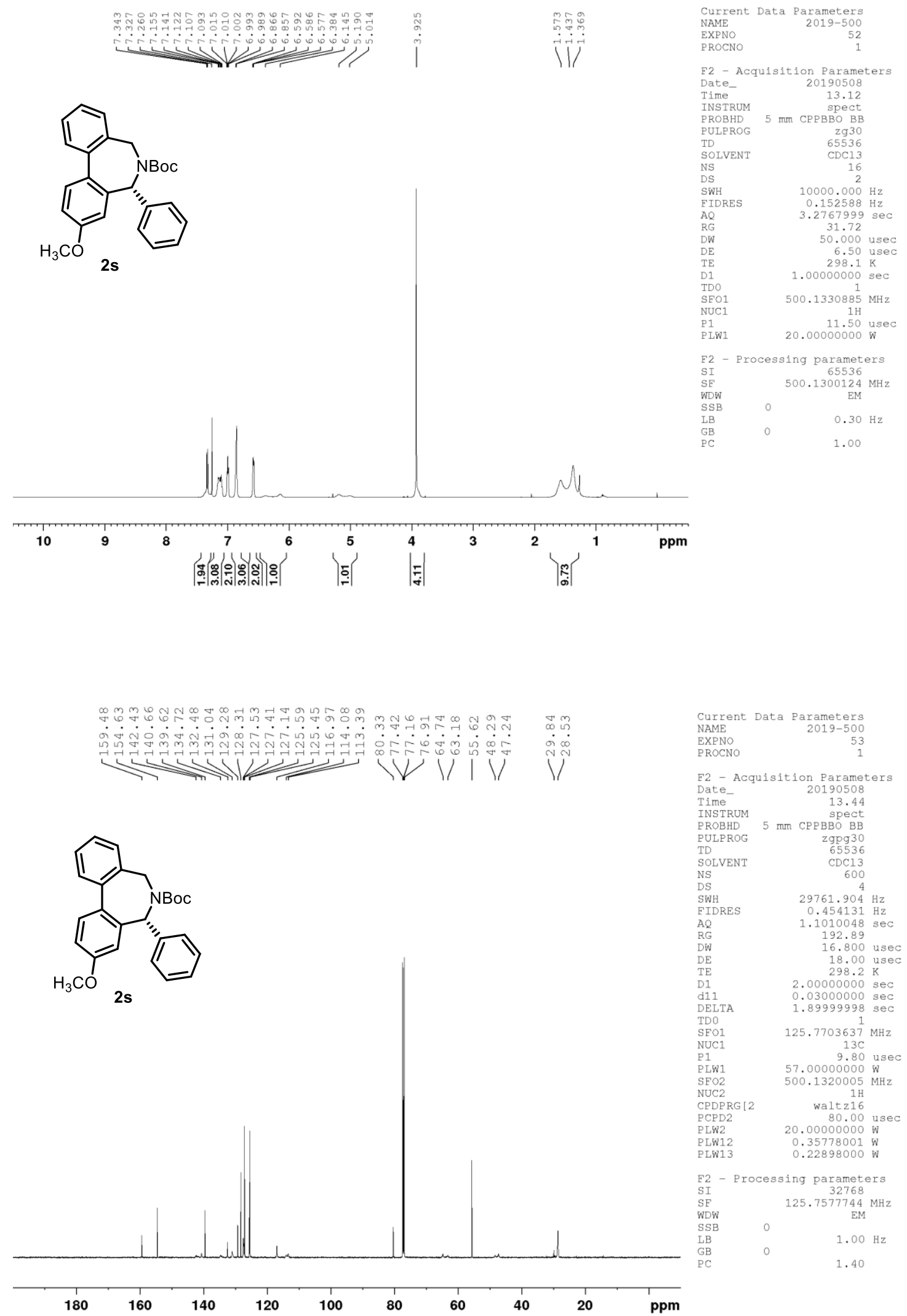




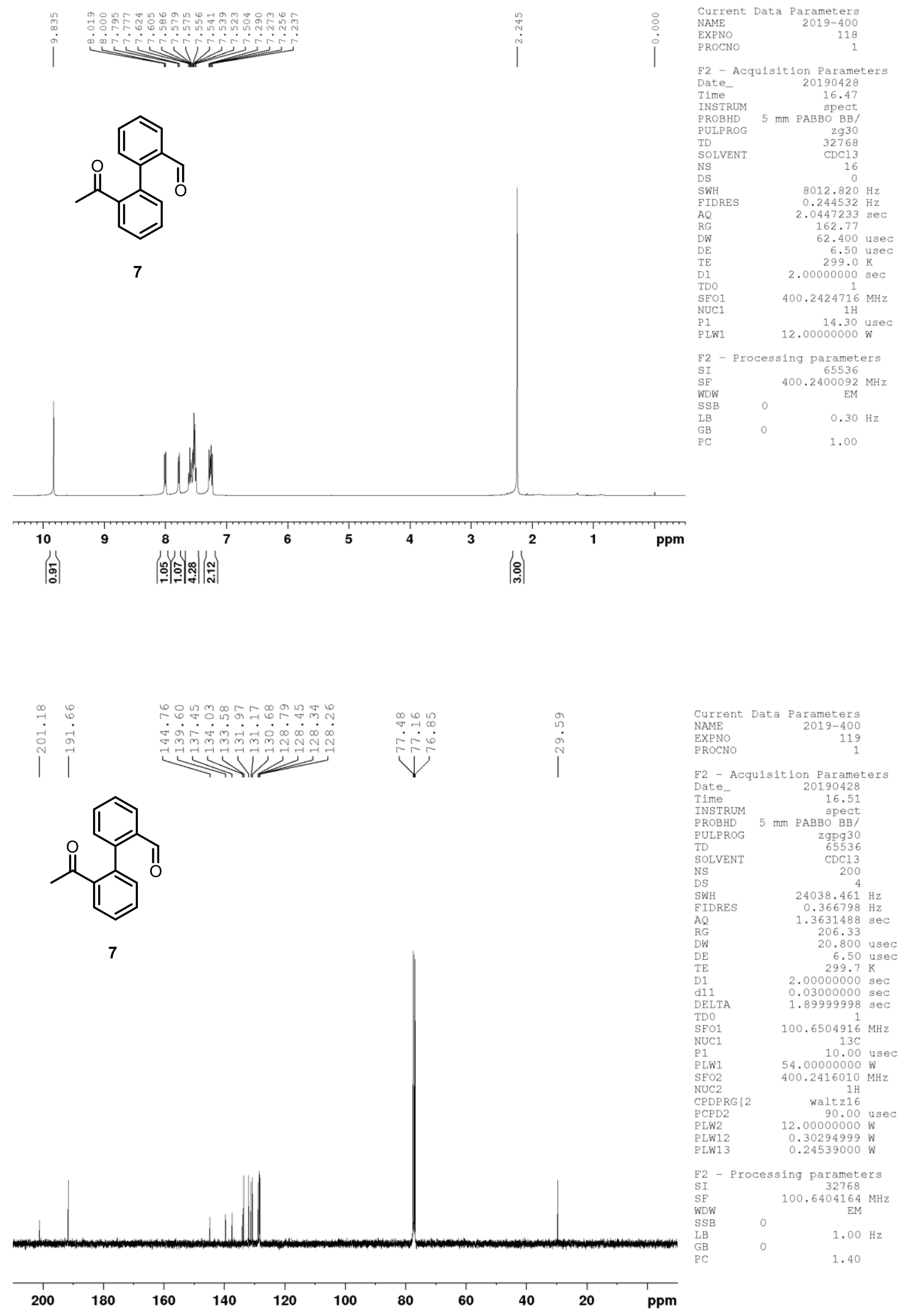



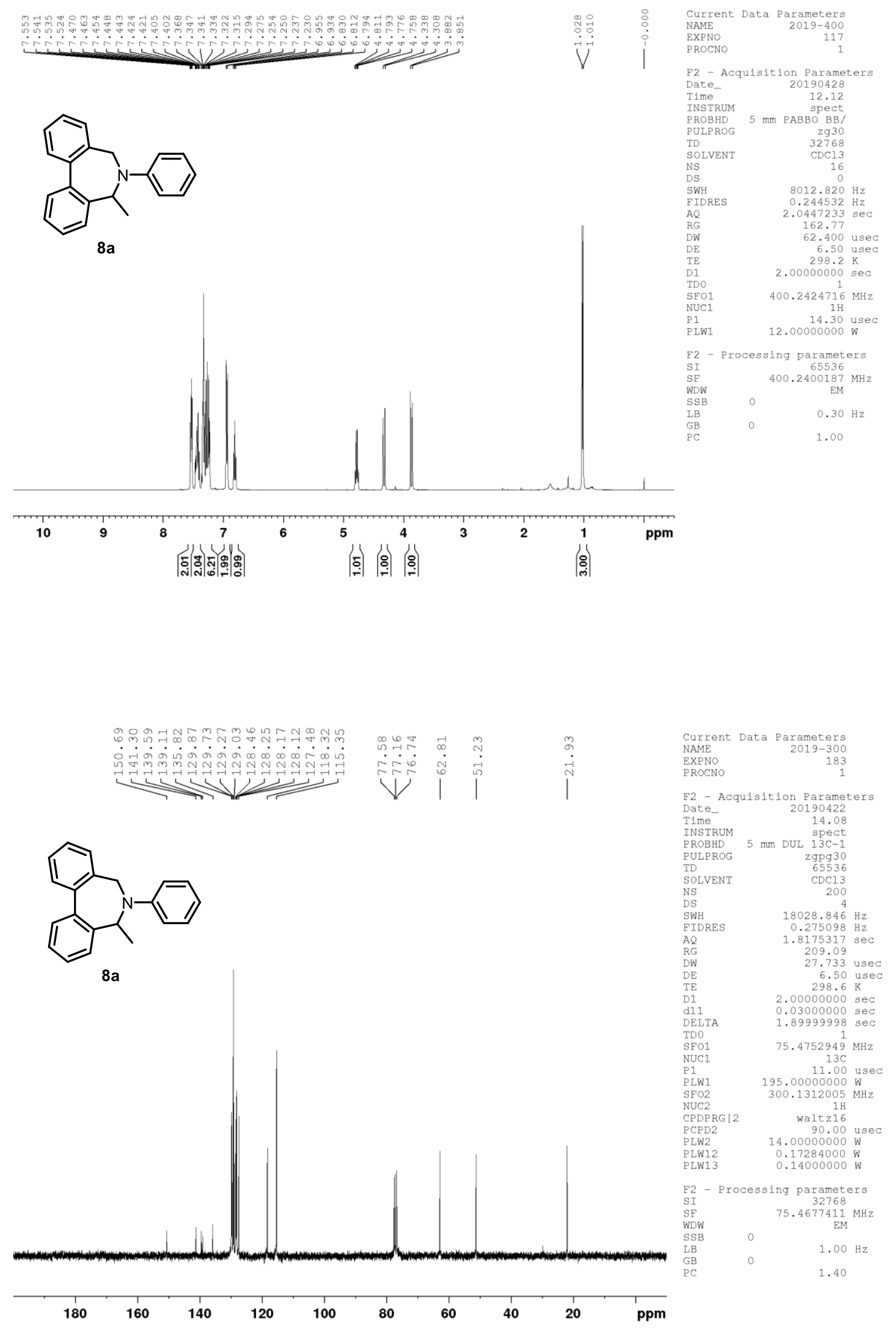


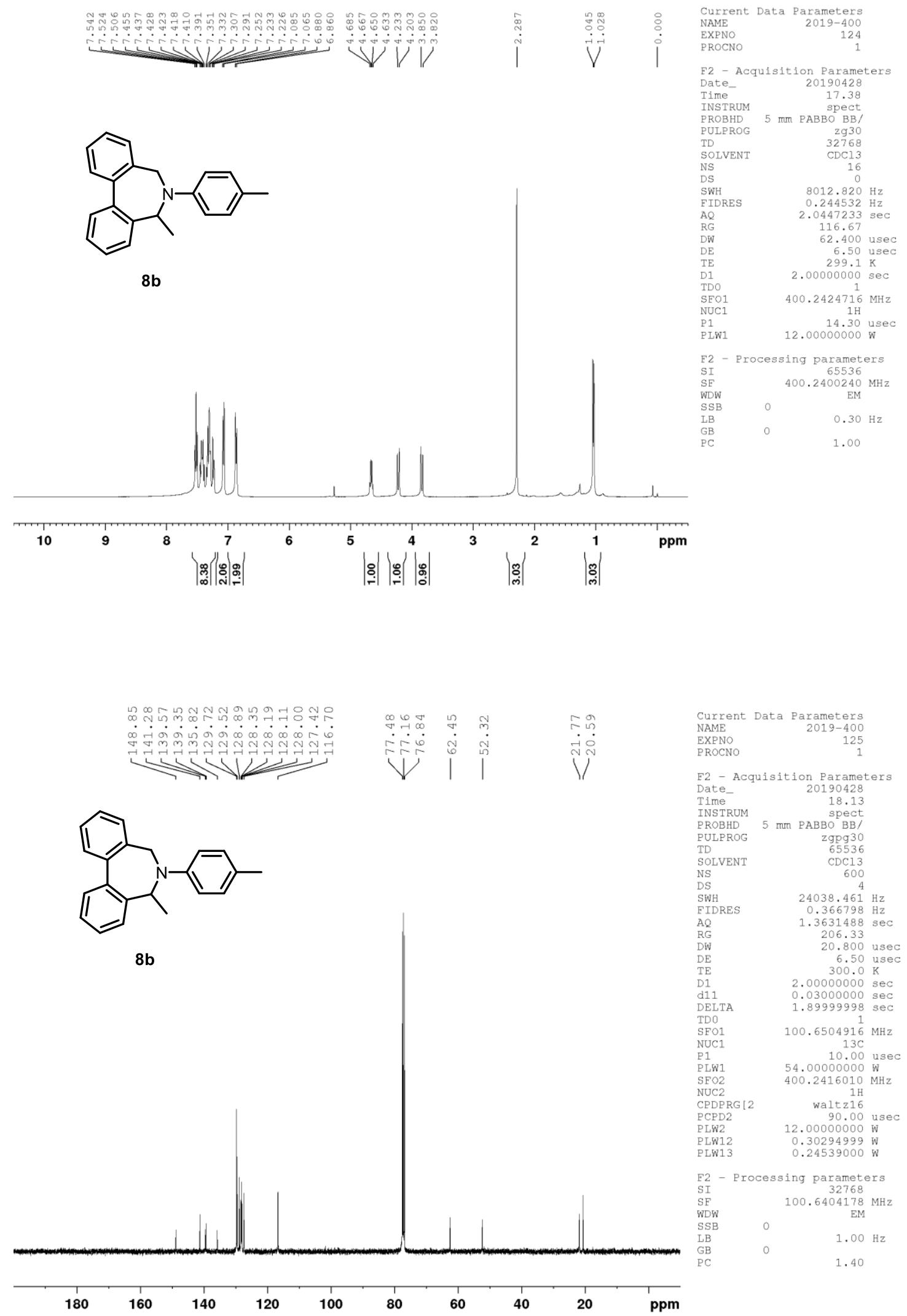




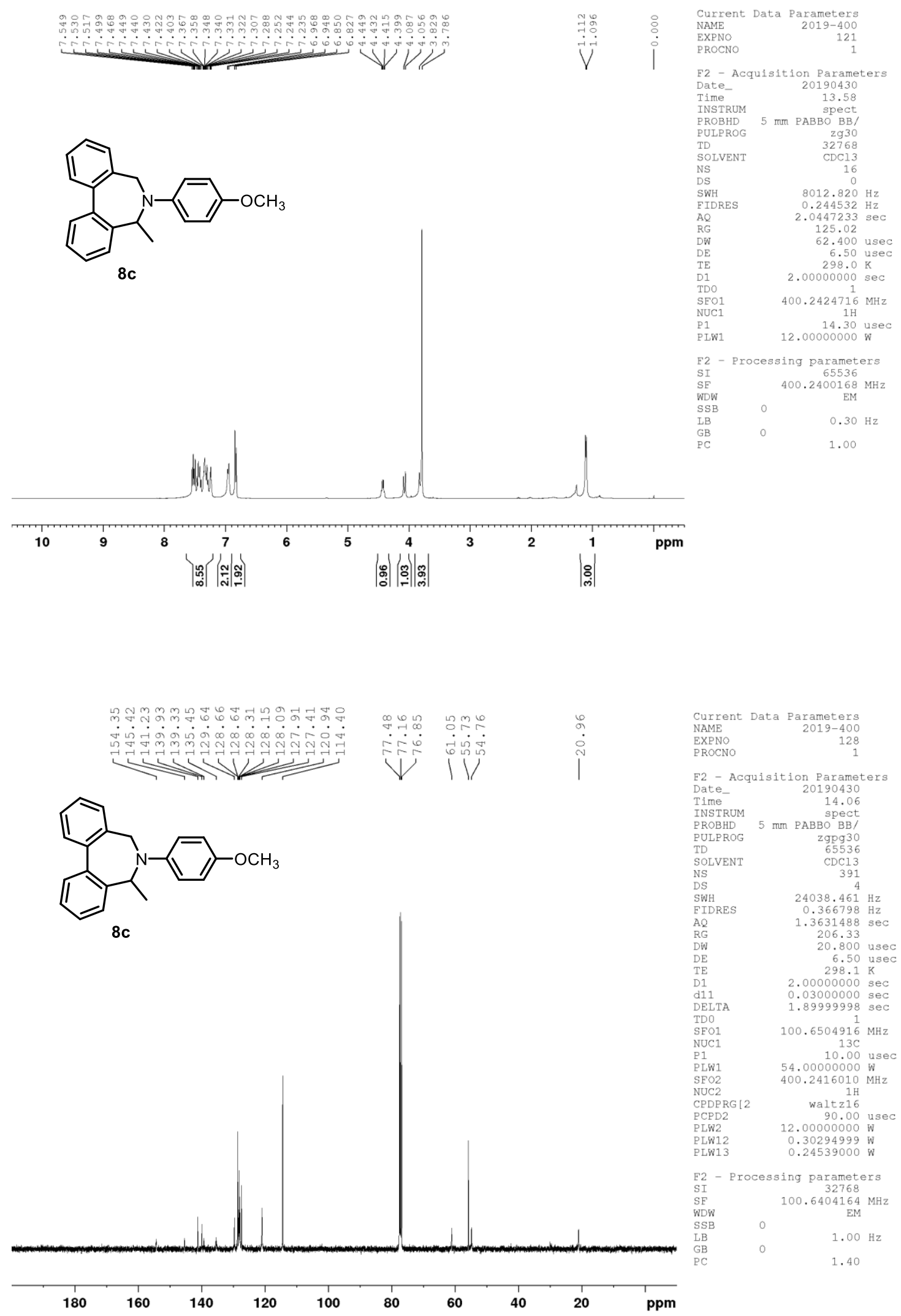



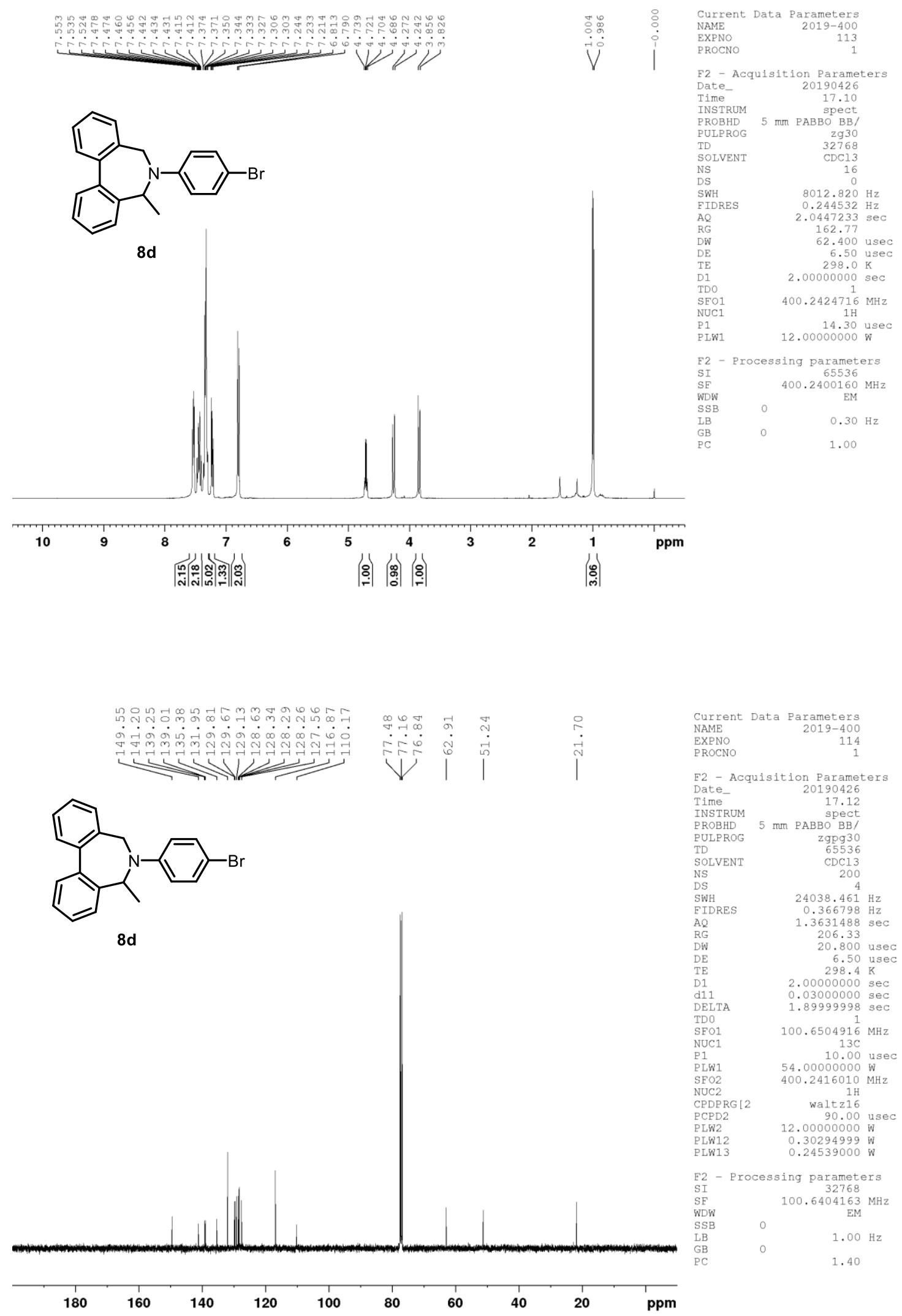

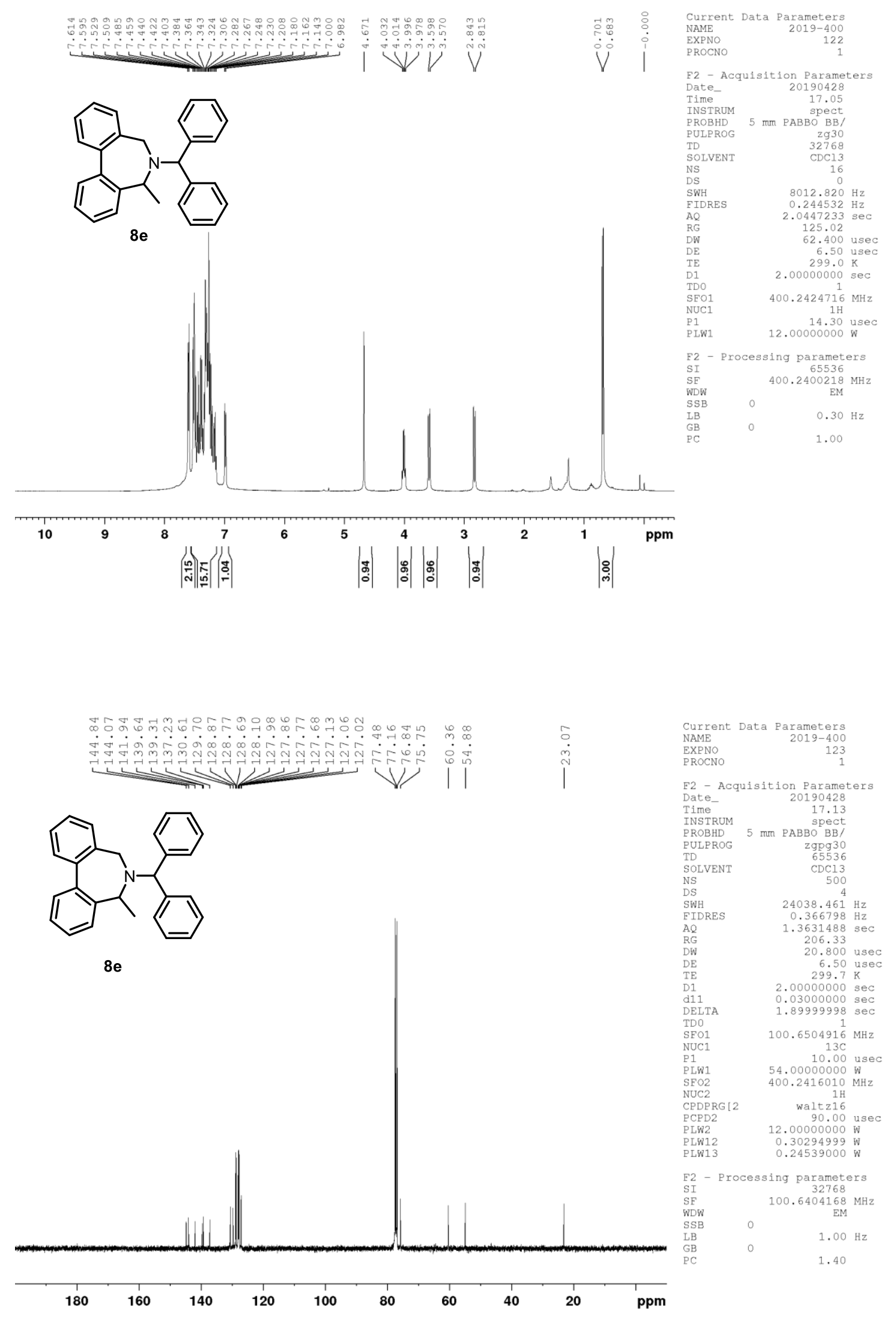

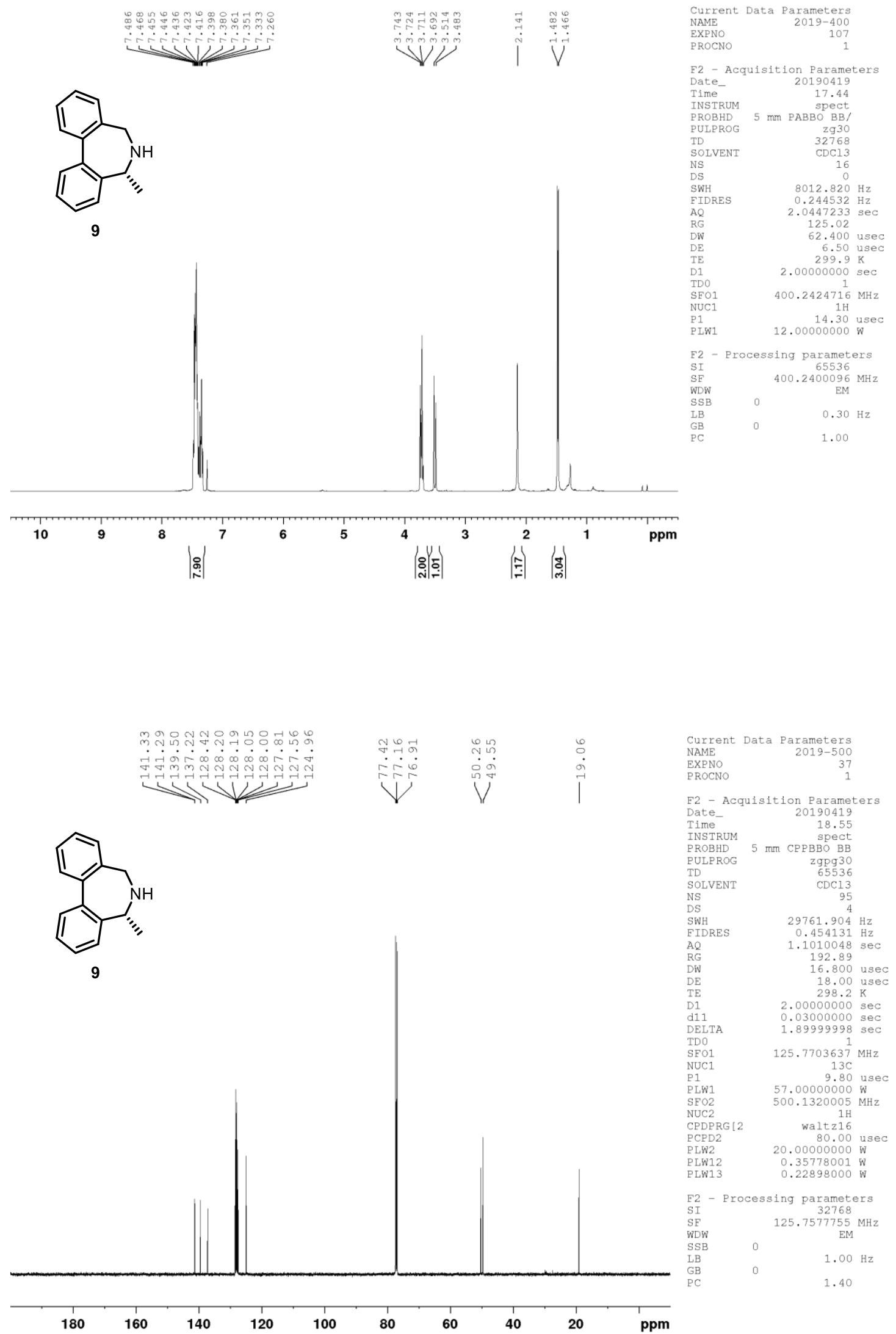


\section{Copy of HPLC spectra}

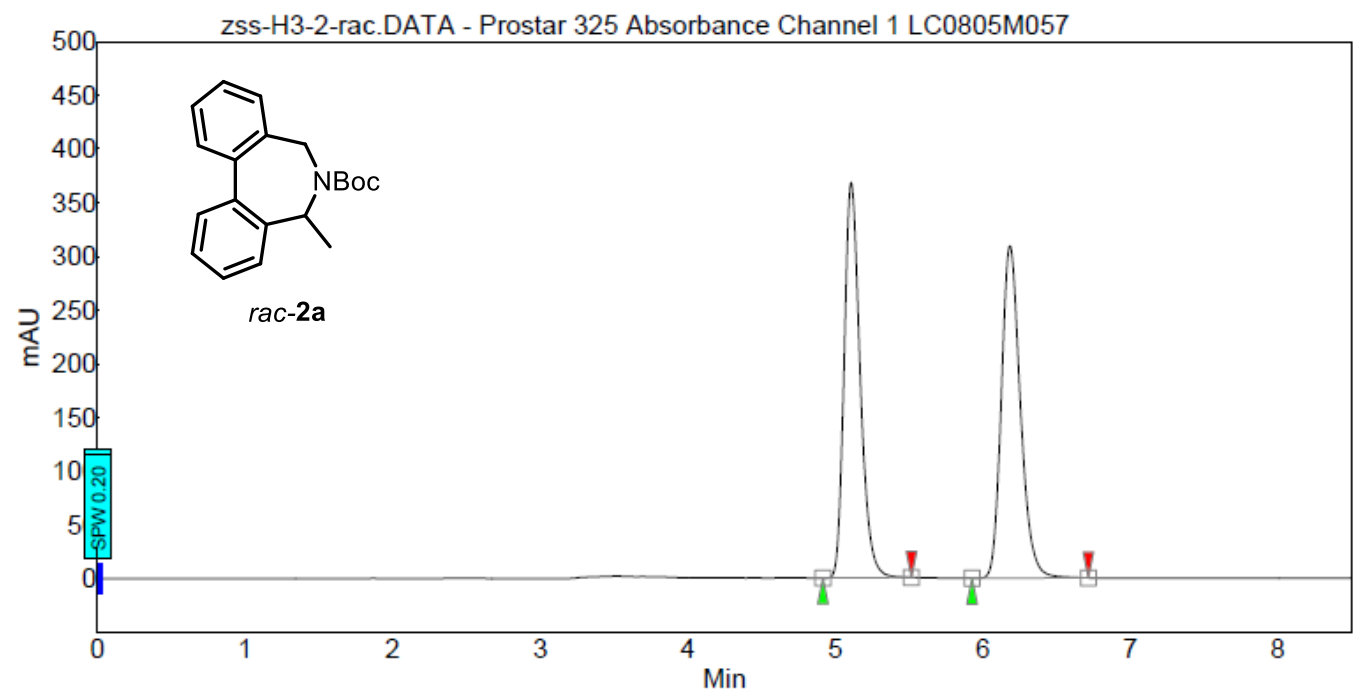

\begin{tabular}{|c|l|r|r|r|r|r|}
\hline Index & 文件名 & $\begin{array}{r}\text { 时间 } \\
\text { [Min] }\end{array}$ & $\begin{array}{r}\text { 数量 } \\
\text { [\% 面积] }\end{array}$ & $\begin{array}{r}\text { 高度 } \\
\text { [mAU] }\end{array}$ & $\begin{array}{r}\text { Area } \\
\text { [mAU.Min] }\end{array}$ & $\begin{array}{r}\text { Area \% } \\
\text { [\%] }\end{array}$ \\
\hline \hline 1 & 末知 & 5.11 & 49.92 & 368.2 & 46.2 & 49.922 \\
\hline 2 & 末知 & 6.18 & 50.08 & 309.0 & 46.4 & 50.078 \\
\hline & & & & & & \\
\hline Total & & & 100.00 & 677.3 & 92.6 & 100.000 \\
\hline
\end{tabular}

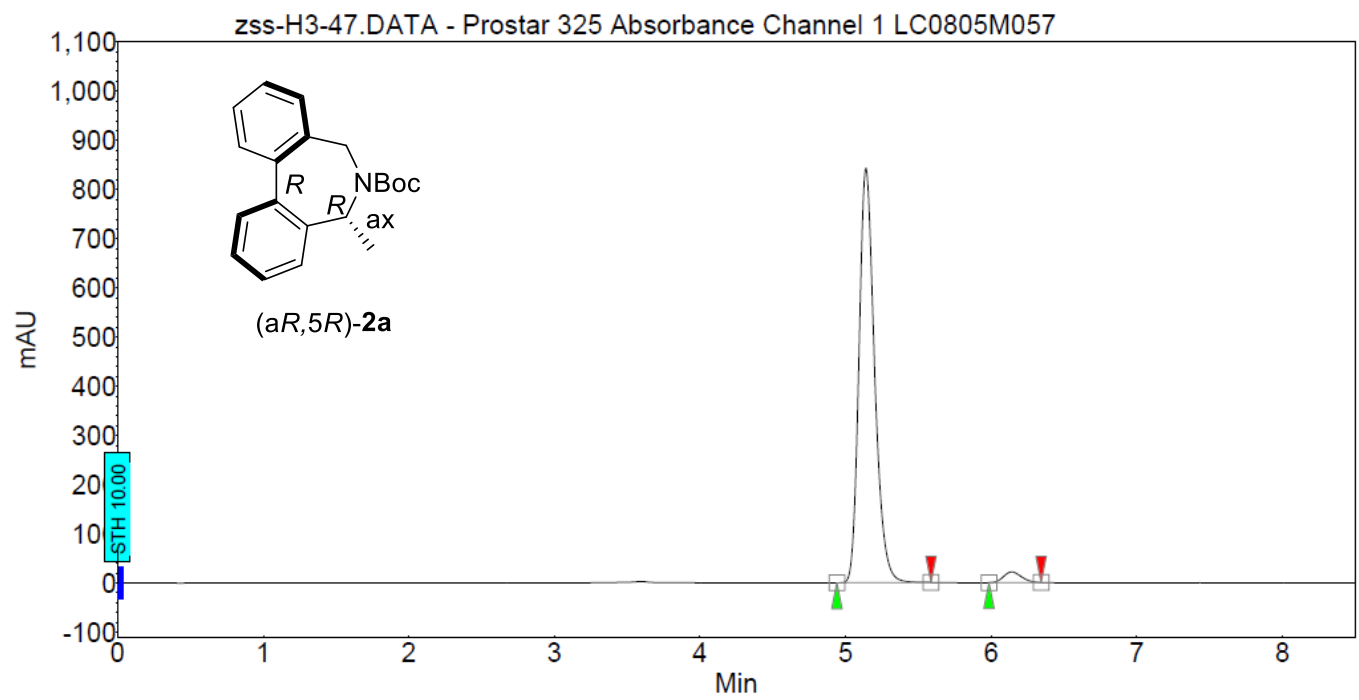

\begin{tabular}{|c|l|r|r|r|r|r|}
\hline Index & 文件名 & $\begin{array}{r}\text { 时间 } \\
{[\text { [Min] }}\end{array}$ & $\begin{array}{r}\text { 数量 } \\
{[\% \text { 面积 }]}\end{array}$ & $\begin{array}{r}\text { 高度 } \\
{[\text { mAU] }}\end{array}$ & $\begin{array}{r}\text { Area } \\
\text { [mAU.Min] }\end{array}$ & $\begin{array}{r}\text { Area \% } \\
{[\%]}\end{array}$ \\
\hline \hline 1 & 未知 & 5.14 & 97.11 & 843.1 & 104.6 & 97.114 \\
\hline 2 & 未知 & 6.14 & 2.89 & 21.8 & 3.1 & 2.886 \\
\hline & & & & & & \\
\hline Total & & & 100.00 & 865.0 & 107.7 & 100.000 \\
\hline
\end{tabular}




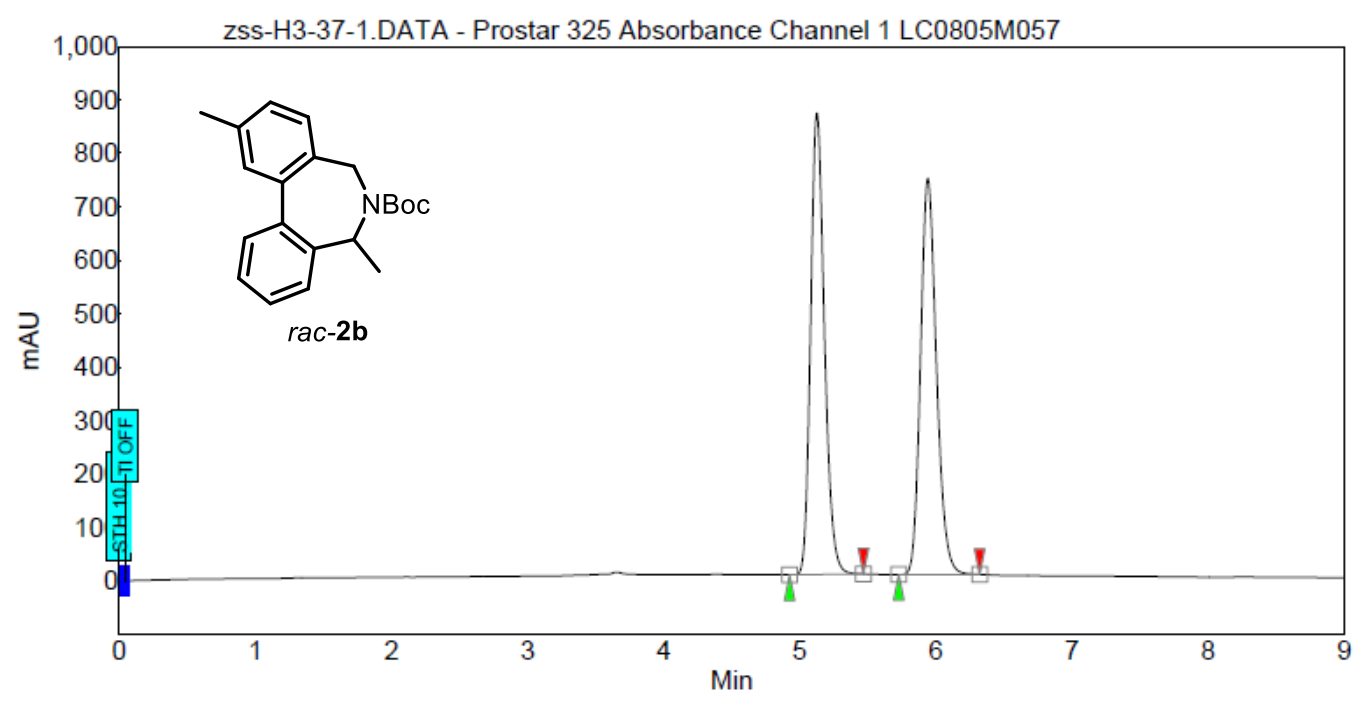

\begin{tabular}{|c|l|r|r|r|r|r|}
\hline \multicolumn{2}{|l|}{ Index 文件名 } & \multicolumn{1}{|c|}{$\begin{array}{l}\text { 时间 } \\
\text { [Min] }\end{array}$} & $\begin{array}{r}\text { 数量 } \\
\text { [\%面积] }\end{array}$ & $\begin{array}{r}\text { 高度 } \\
\text { [mAU] }\end{array}$ & $\begin{array}{r}\text { Area } \\
\text { [mAU.Min] }\end{array}$ & $\begin{array}{r}\text { Area \% } \\
\text { [\%] }\end{array}$ \\
\hline \hline 1 & 末知 & 5.13 & 50.26 & 863.4 & 101.5 & 50.260 \\
\hline 2 & 末知 & 5.94 & 49.74 & 741.9 & 100.5 & 49.740 \\
\hline & & & & & & \\
\hline Total & & & 100.00 & 1605.3 & 202.0 & 100.000 \\
\hline
\end{tabular}

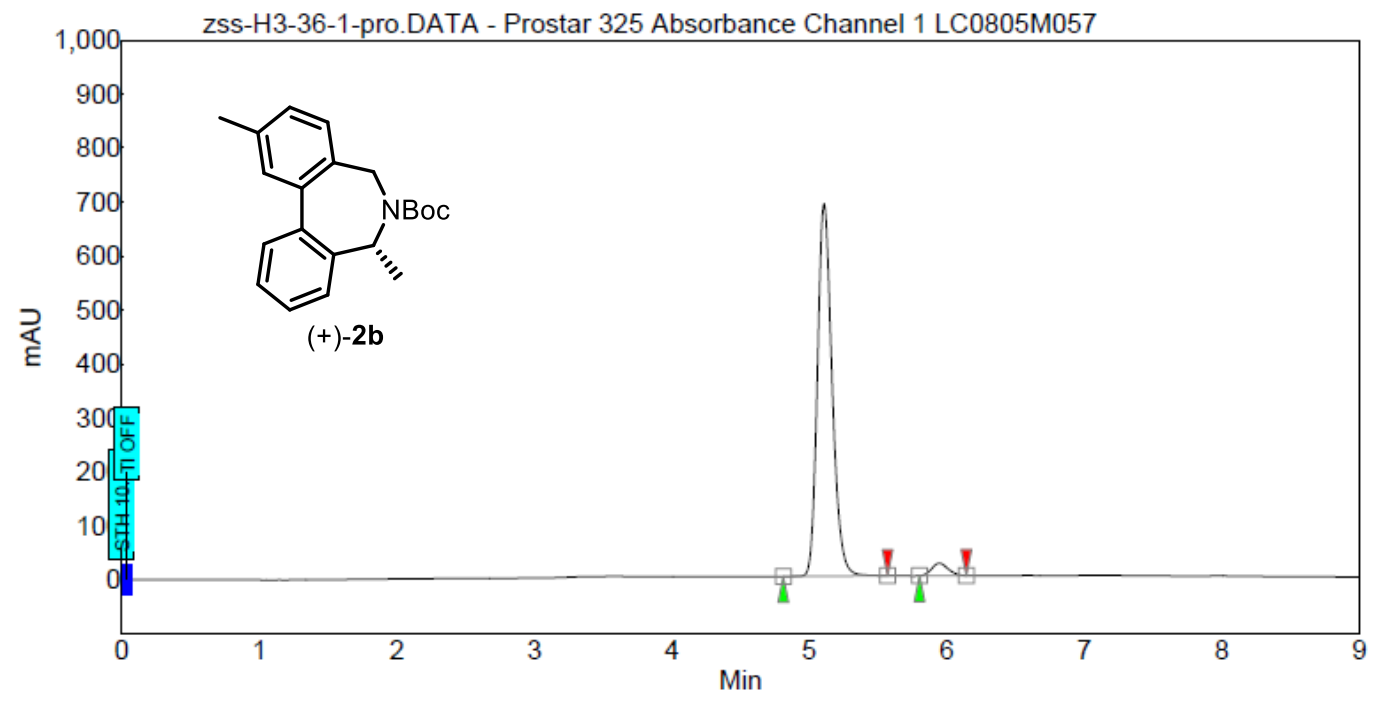

\begin{tabular}{|c|c|c|c|c|c|c|}
\hline Index & 文件名 & $\begin{array}{l}\text { 时间 } \\
\text { [Min] }\end{array}$ & [\% 面数量 & $\begin{array}{r}\text { 高度 } \\
\text { [mAU] }\end{array}$ & $\begin{array}{r}\text { Area } \\
\text { [mAU.Min] }\end{array}$ & $\begin{array}{r}\text { Area \% } \\
{[\%]} \\
\end{array}$ \\
\hline 1 & 未知 & 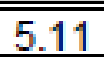 & 96.43 & 690.3 & 85.3 & 96.425 \\
\hline 2 & 末知 & 5.94 & 3.57 & 23.0 & 3.2 & 3.575 \\
\hline Total & & & 10000 & 7133 & 88.4 & 100 \\
\hline
\end{tabular}




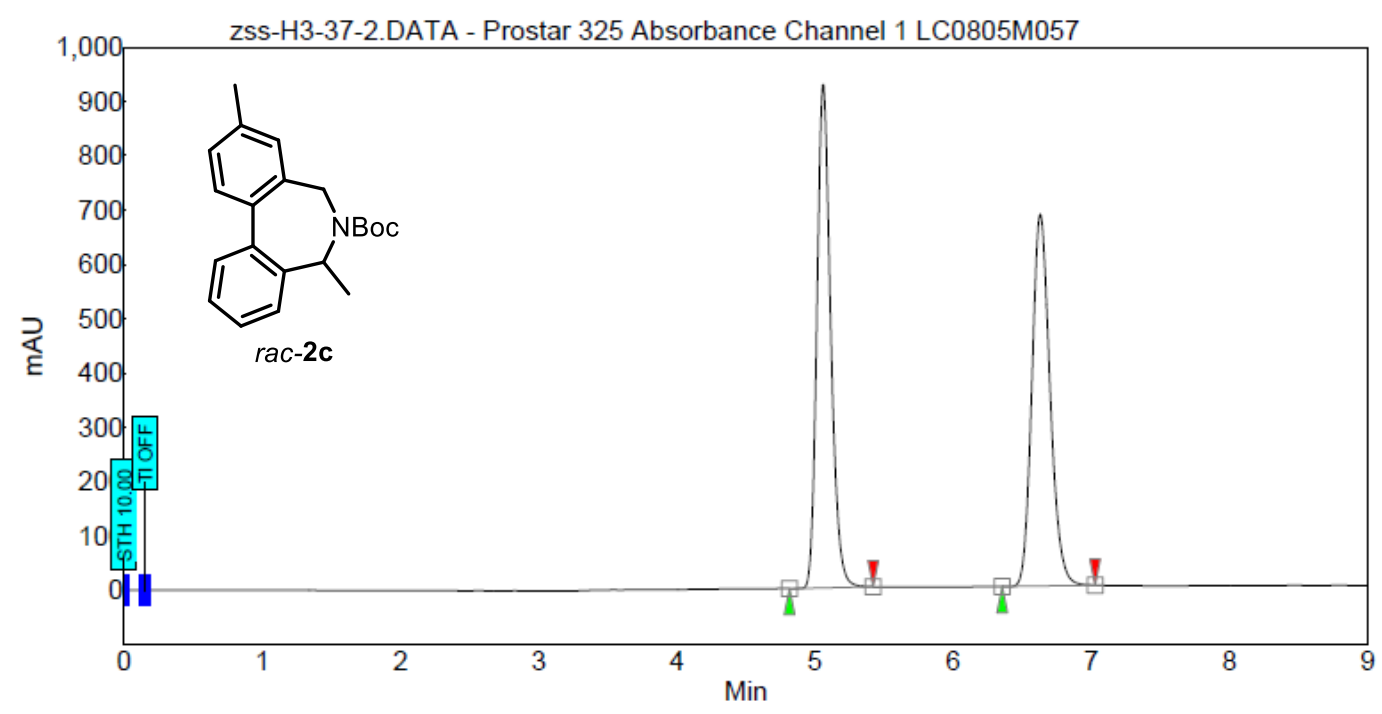

\begin{tabular}{|c|l|r|r|r|r|r|}
\hline \multicolumn{2}{|l|}{ Index 文件名 } & \multicolumn{1}{|c|}{$\begin{array}{l}\text { 时间 } \\
\text { [Min] }\end{array}$} & $\begin{array}{r}\text { 数量 } \\
\text { [\% 面积] }\end{array}$ & $\begin{array}{r}\text { 高度 } \\
\text { [mAU] }\end{array}$ & $\begin{array}{r}\text { Area } \\
\text { [mAU.Min] }\end{array}$ & $\begin{array}{r}\text { Area \% } \\
\text { [\%] }\end{array}$ \\
\hline \hline 1 & 末知 & 5.06 & 50.69 & 926.8 & 107.0 & 50.686 \\
\hline 2 & 末知 & 6.63 & 49.31 & 685.3 & 104.1 & 49.314 \\
\hline & & & & & & \\
\hline Total & & & 100.00 & 1612.0 & 211.2 & 100.000 \\
\hline
\end{tabular}

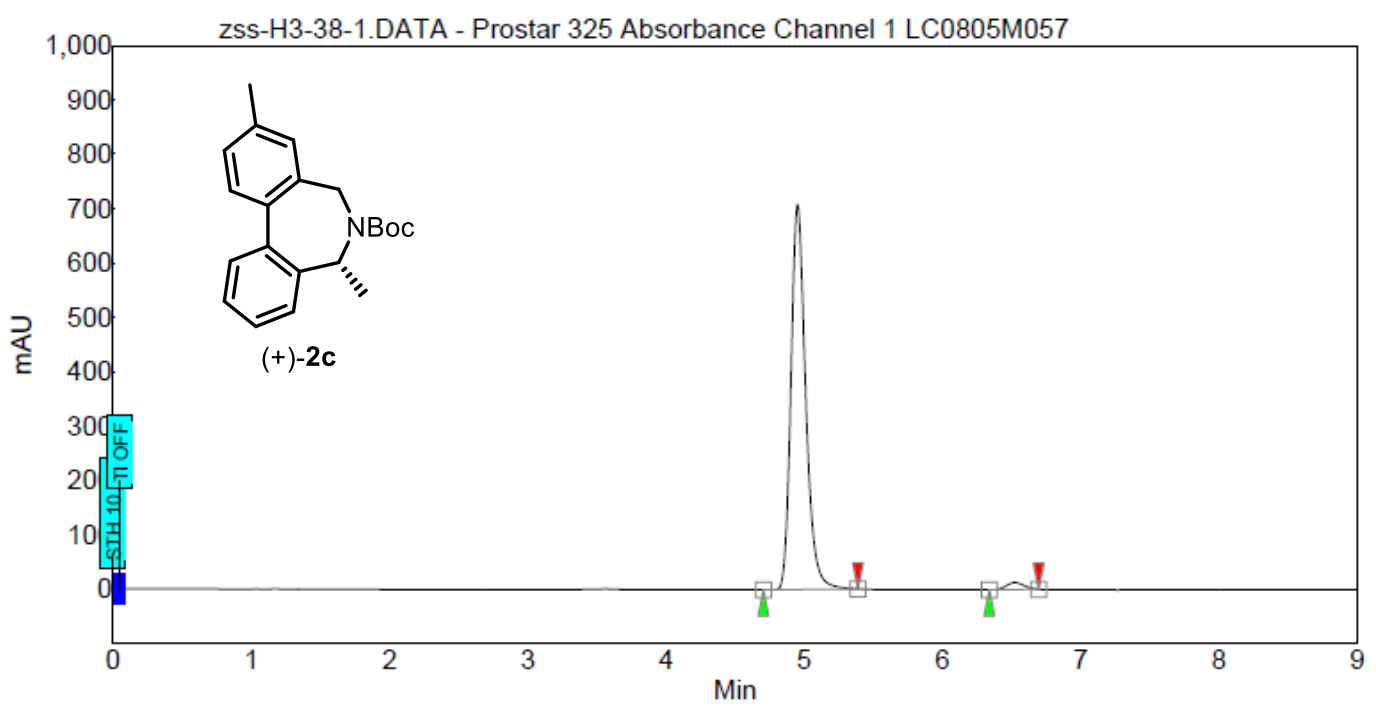

\begin{tabular}{|c|l|r|r|r|r|r|}
\hline \multicolumn{2}{|l|}{ Index 文件名 } & \multicolumn{1}{c|}{$\begin{array}{r}\text { 时间 } \\
\text { [Min] }\end{array}$} & $\begin{array}{r}\text { 数量 } \\
\text { [\% 面积] }\end{array}$ & $\begin{array}{r}\text { 高度 } \\
\text { [mAU] }\end{array}$ & $\begin{array}{r}\text { Area } \\
\text { [mAU.Min] }\end{array}$ & $\begin{array}{r}\text { Area \% } \\
{[\%]}\end{array}$ \\
\hline \hline 1 & 未知 & 4.95 & 97.88 & 708.6 & 87.4 & 97.875 \\
\hline 2 & 未知 & 6.52 & 2.12 & 12.8 & 1.9 & 2.125 \\
\hline & & & & & & \\
\hline Total & & & 100.00 & 721.4 & 89.3 & 100.000 \\
\hline
\end{tabular}




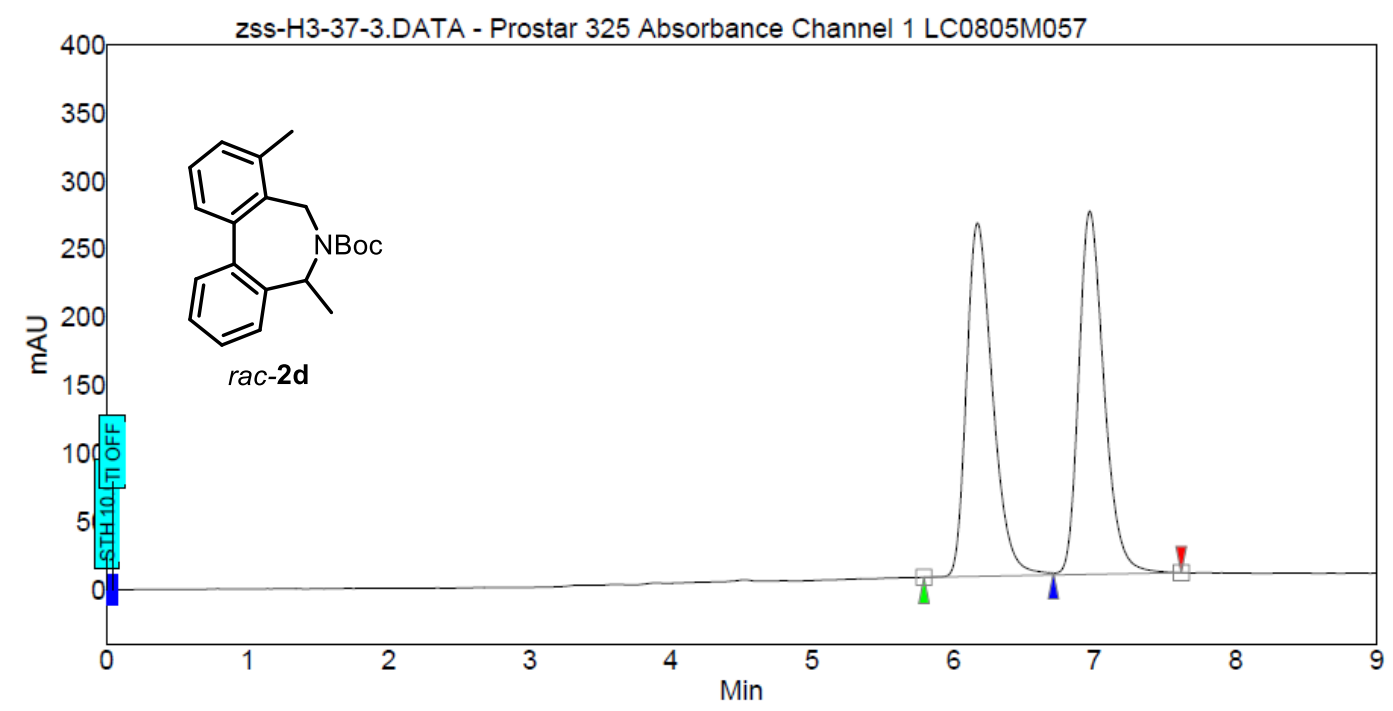

\begin{tabular}{|c|c|c|c|c|c|c|}
\hline Index & 文件名 & $\begin{array}{l}\text { 时间 } \\
\text { [Min] }\end{array}$ & [\% 面积 & $\begin{array}{r}\text { 高度 } \\
\text { [mAU] }\end{array}$ & $\begin{array}{r}\text { Area } \\
\text { [mAU.Min] }\end{array}$ & $\begin{array}{r}\text { Area \% } \\
{[\%]}\end{array}$ \\
\hline 1 & 末知 & 6.17 & 50.01 & 259.5 & 55.6 & 50.012 \\
\hline 2 & 末知 & 6.97 & 49.99 & 266.8 & 55.6 & 49.988 \\
\hline Total & & & 100.00 & 526.3 & 111.3 & 100.000 \\
\hline
\end{tabular}

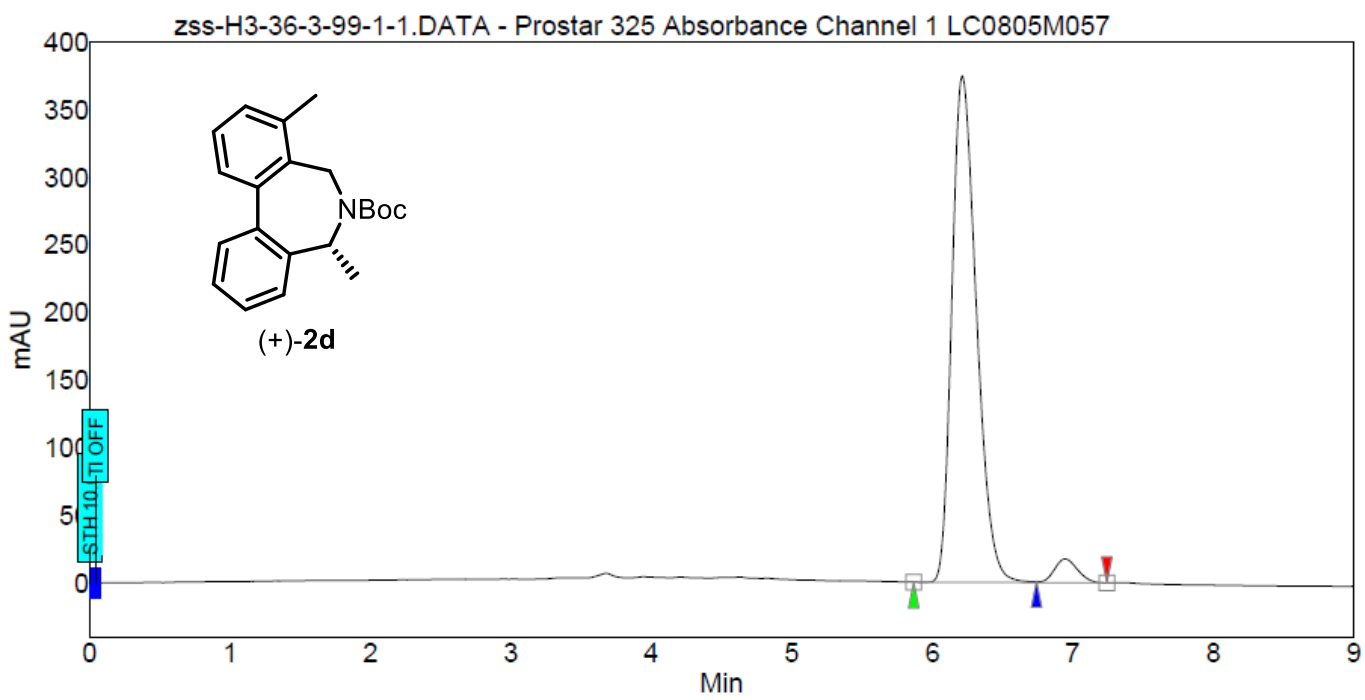

\begin{tabular}{|c|c|c|c|c|c|c|}
\hline Index & 文件名 & $\begin{array}{l}\text { 时间 } \\
\text { [Min] }\end{array}$ & [\% 面积] & $\begin{array}{r}\text { 高度 } \\
\text { [mAU] }\end{array}$ & $\begin{array}{r}\text { Area } \\
\text { [mAU.Min] }\end{array}$ & $\begin{array}{r}\text { Area \% } \\
{[\%]}\end{array}$ \\
\hline 1 & 末知 & 6.21 & 95.74 & 374.6 & 75.8 & 95.742 \\
\hline 2 & 末知 & 6.95 & 4.26 & 17.6 & 3.4 & 4.258 \\
\hline Total & & & 100.00 & 392.2 & 79.2 & 100.000 \\
\hline
\end{tabular}




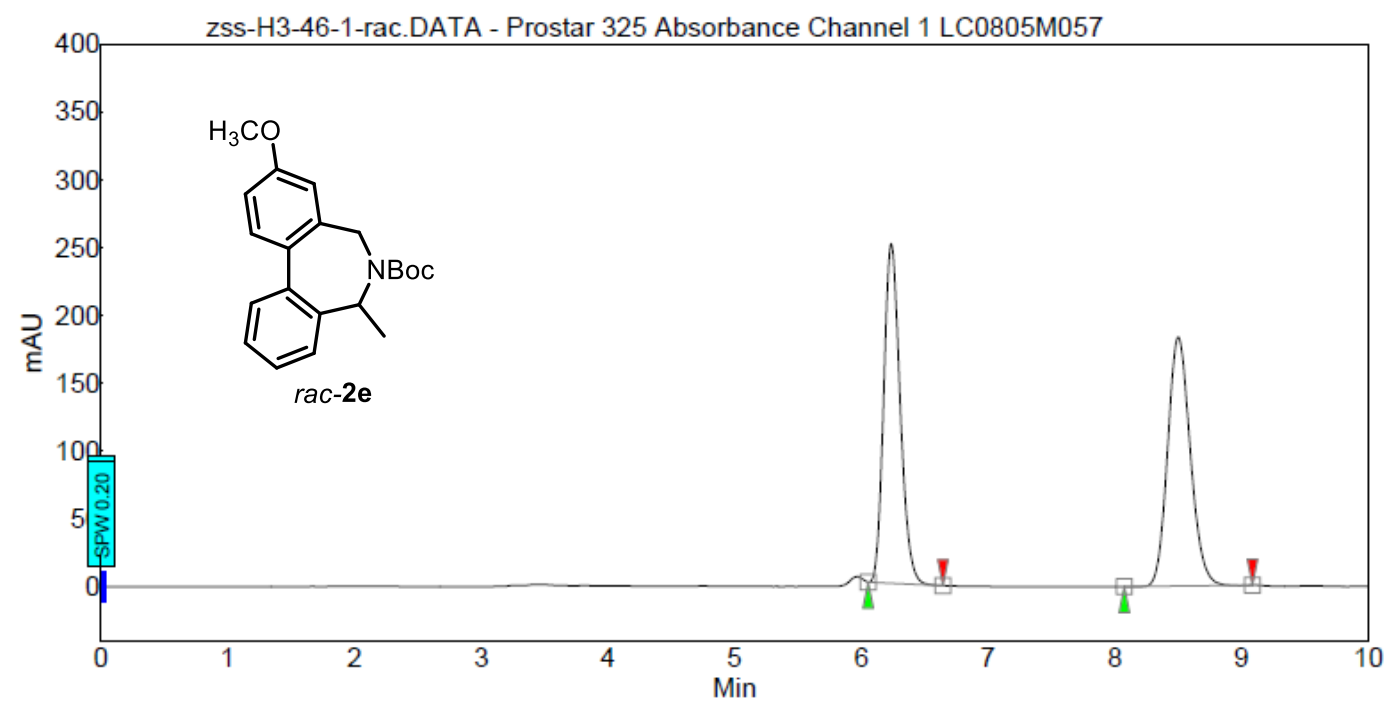

\begin{tabular}{|c|l|r|r|r|r|r|}
\hline Index & 文件名 & $\begin{array}{r}\text { 时间 } \\
{[\mathrm{Min}]}\end{array}$ & $\begin{array}{r}\text { 数量 } \\
\text { [\% 面积] }\end{array}$ & $\begin{array}{r}\text { 高度 } \\
{[\mathrm{mAU}]}\end{array}$ & $\begin{array}{r}\text { Area } \\
\text { [mAU.Min] }\end{array}$ & $\begin{array}{r}\text { Area \% } \\
\text { [\%] }\end{array}$ \\
\hline \hline 1 & 末知 & 6.23 & 49.69 & 250.2 & 37.5 & 49.691 \\
\hline 2 & 末知 & 8.50 & 50.31 & 183.4 & 38.0 & 50.309 \\
\hline & & & & & & \\
\hline Total & & & 100.00 & 433.6 & 75.5 & 100.000 \\
\hline
\end{tabular}

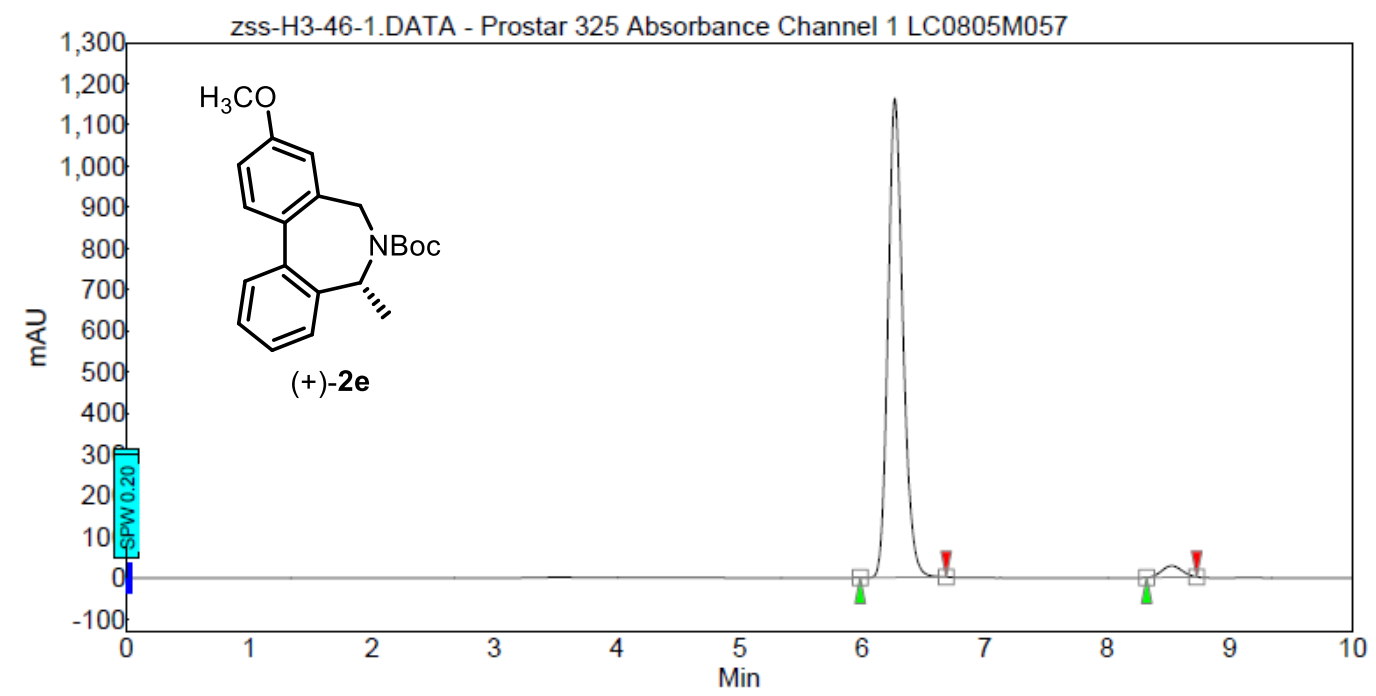

\begin{tabular}{|c|c|c|c|c|c|c|}
\hline Index & 文件名 & $\begin{array}{l}\text { 时间 } \\
\text { [Min] }\end{array}$ & [\% 面积] & $\begin{array}{r}\text { 高度 } \\
\text { [mAU] }\end{array}$ & $\begin{array}{r}\text { Area } \\
\text { [mAU.Min] }\end{array}$ & $\begin{array}{r}\text { Area } \% \\
{[\%]}\end{array}$ \\
\hline 1 & 末知 & 6.27 & 96.88 & 1163.0 & 164.6 & 96.879 \\
\hline 2 & 末知 & 8.53 & 3.12 & 27.8 & 5.3 & 3.121 \\
\hline Total & & & 10000 & 11907 & 1700 & 100000 \\
\hline
\end{tabular}




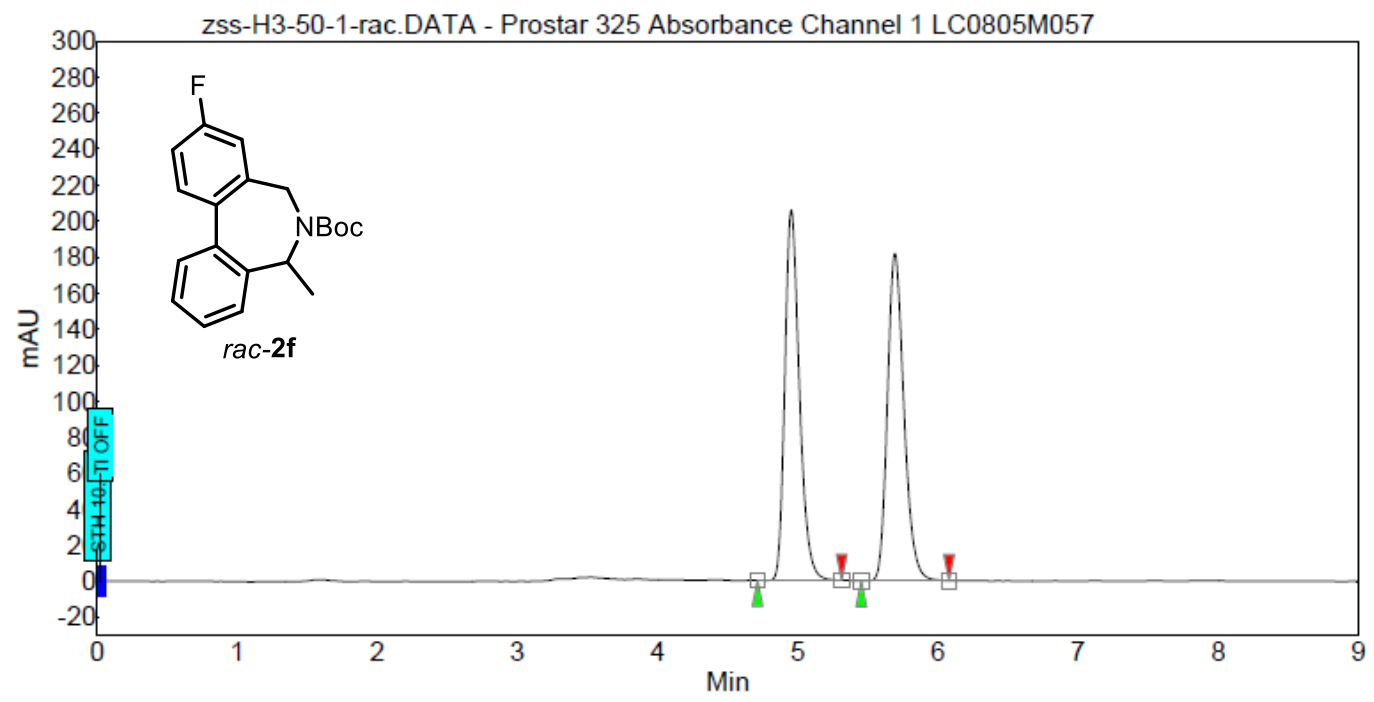

\begin{tabular}{|c|c|c|c|c|c|c|}
\hline Index & 文件名 & $\begin{array}{r}\begin{array}{l}\text { 时间 } \\
\text { [Min] }\end{array} \\
\end{array}$ & [\% 面数量 & $\begin{array}{r}\text { 高度 } \\
\text { [mAU] }\end{array}$ & $\begin{array}{r}\text { Area } \\
\text { [mAU.Min] }\end{array}$ & $\begin{array}{r}\text { Area \% } \\
{[\%]}\end{array}$ \\
\hline 1 & 本知 & 4.95 & 49.89 & 205.7 & 24.7 & 49.890 \\
\hline 2 & 末知 & 5.69 & 50.11 & 181.7 & 24.8 & 50.110 \\
\hline Total & & & 100.00 & 387.4 & 49.6 & 100.000 \\
\hline
\end{tabular}

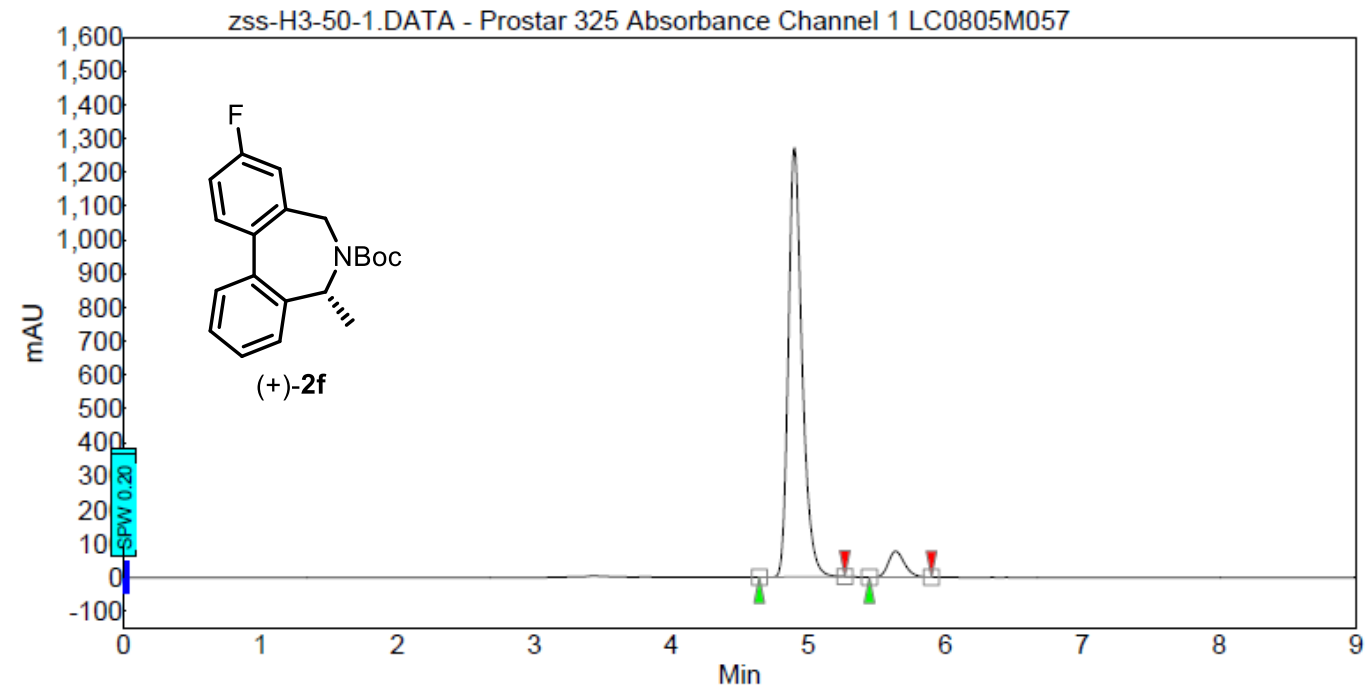

\begin{tabular}{|c|lr|r|r|r|r|}
\hline Index & 文件名 & $\begin{array}{r}\text { 时间 } \\
\text { [Min] }\end{array}$ & $\begin{array}{r}\text { 数量 } \\
\text { [\% 面积] }\end{array}$ & \multicolumn{1}{|c|}{$\begin{array}{r}\text { 高度 } \\
\text { [mAU] }\end{array}$} & $\begin{array}{r}\text { Area } \\
\text { [mAU.Min] }\end{array}$ & $\begin{array}{r}\text { Area \% } \\
\text { [\%] }\end{array}$ \\
\hline \hline 1 & 末知 & 4.90 & 93.37 & 1269.9 & 147.8 & 93.371 \\
\hline 2 & 本知 & 5.63 & 6.63 & 76.9 & 10.5 & 6.629 \\
\hline & & & & & & \\
\hline Total & & & 100.00 & 1346.8 & 158.3 & 100.000 \\
\hline
\end{tabular}




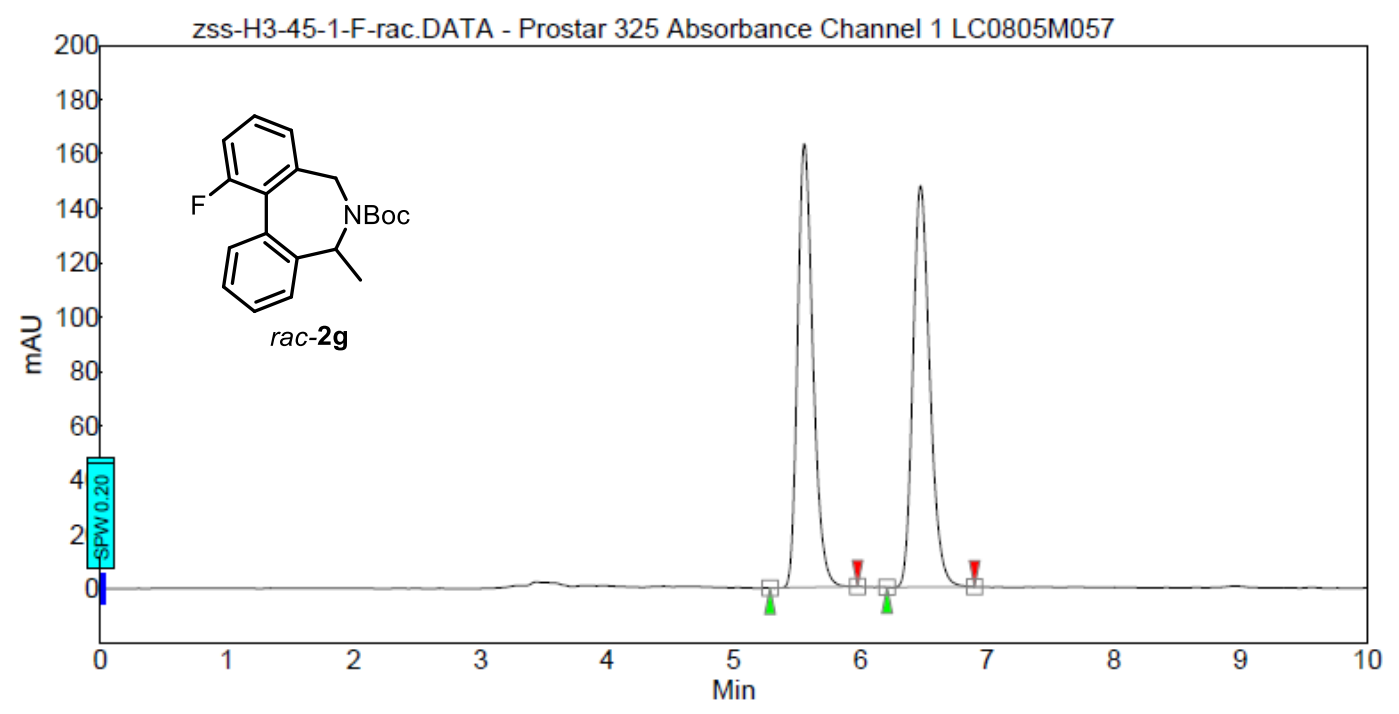

\begin{tabular}{|c|l|r|r|r|r|r|}
\hline Index & 文件名 & $\begin{array}{r}\text { 时间 } \\
{[\mathrm{Min}]}\end{array}$ & $\begin{array}{r}\text { 数量 } \\
\text { [\% 面积] }\end{array}$ & $\begin{array}{r}\text { 高度 } \\
{[\mathrm{mAU}]}\end{array}$ & $\begin{array}{r}\text { Area } \\
\text { [mAU.Min] }\end{array}$ & $\begin{array}{r}\text { Area \% } \\
\text { [\%] }\end{array}$ \\
\hline \hline 1 & 末知 & 5.55 & 50.02 & 163.3 & 23.0 & 50.018 \\
\hline 2 & 末知 & 6.47 & 49.98 & 147.7 & 23.0 & 49.982 \\
\hline & & & & & & \\
\hline Total & & & 100.00 & 311.0 & 45.9 & 100.000 \\
\hline
\end{tabular}

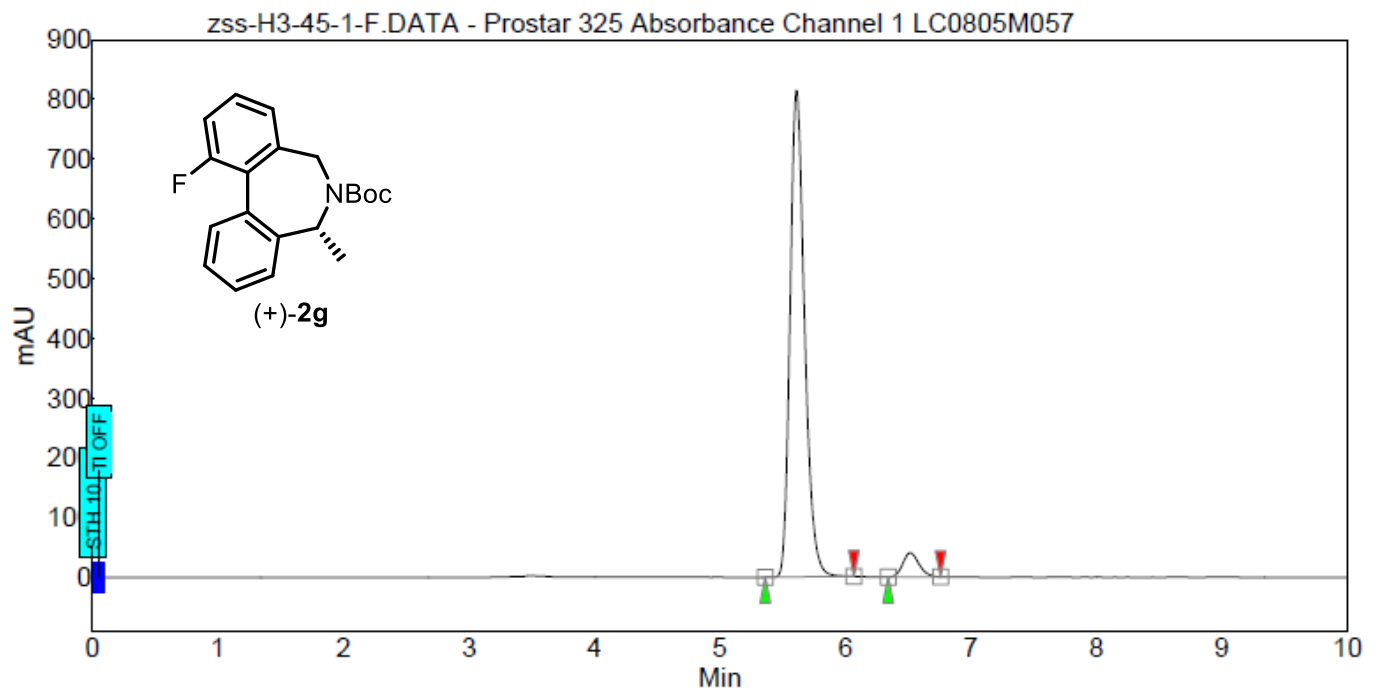

\begin{tabular}{|c|c|c|c|c|c|c|}
\hline Index & 文件名 & $\begin{array}{l}\text { 时间 } \\
\text { [Min] }\end{array}$ & [\% 面积] & $\begin{array}{c}\text { 高度 } \\
\text { [mAU] }\end{array}$ & $\begin{array}{r}\text { Area } \\
\text { [mAU.Min] }\end{array}$ & $\begin{array}{r}\text { Area \% } \\
{[\%]}\end{array}$ \\
\hline 1 & 末知 & 5.61 & 94.77 & 814.6 & 109.3 & 94.767 \\
\hline 2 & 未知 & 6.51 & 5.23 & 40.4 & 6.0 & 5.233 \\
\hline & & & & & & \\
\hline Total & & & 100.00 & 855.0 & 115.3 & 100.000 \\
\hline
\end{tabular}




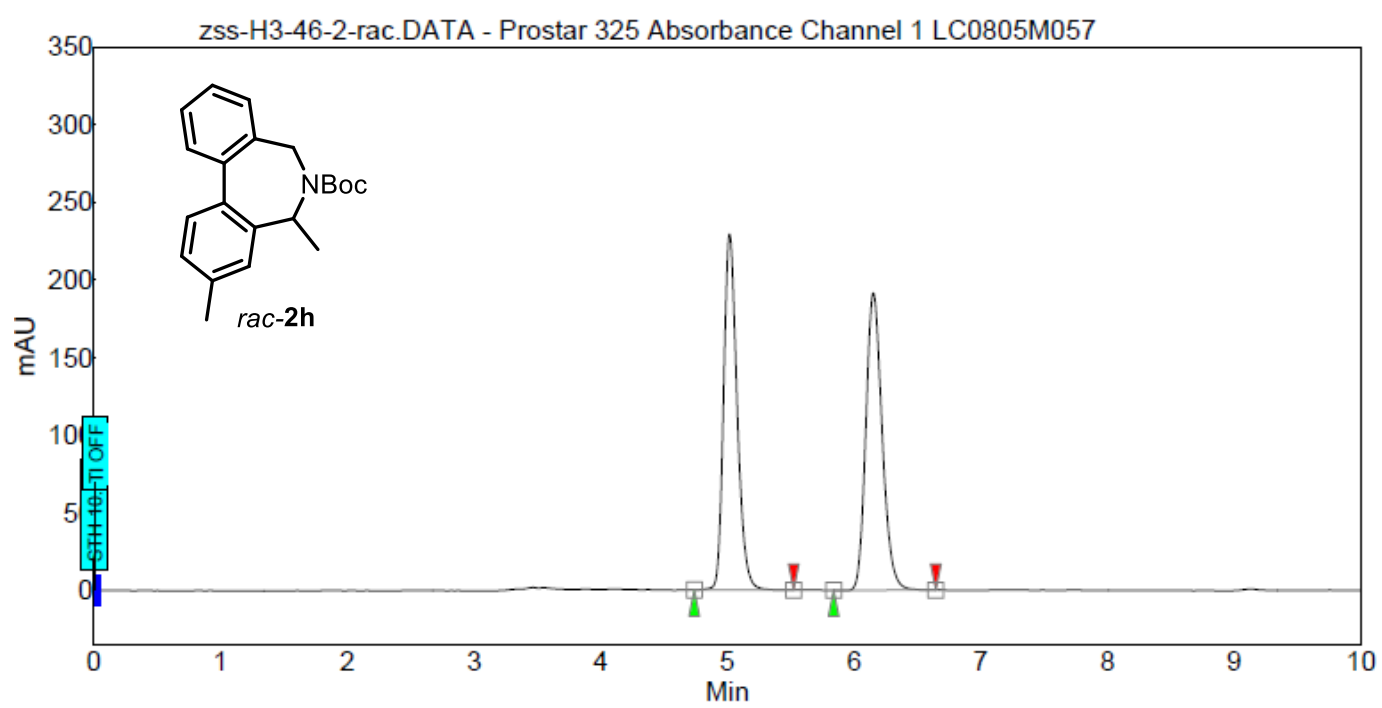

\begin{tabular}{|c|c|c|c|c|c|c|}
\hline Index & 文件名 & $\begin{array}{l}\text { 时间 } \\
\text { [Min] }\end{array}$ & [\% 面积] & $\begin{array}{r}\text { 高度 } \\
\text { [mAU] }\end{array}$ & $\begin{array}{r}\text { Area } \\
\text { [mAU.Min] }\end{array}$ & $\begin{array}{r}\text { Area \% } \\
{[\%]}\end{array}$ \\
\hline 1 & 本知 & 5.01 & 49.73 & 229.1 & 28.3 & 49.733 \\
\hline 2 & 末知 & 6.15 & 50.27 & 191.4 & 28.6 & 50.267 \\
\hline Total & & & 100.00 & 420.5 & 56.9 & 100.000 \\
\hline
\end{tabular}

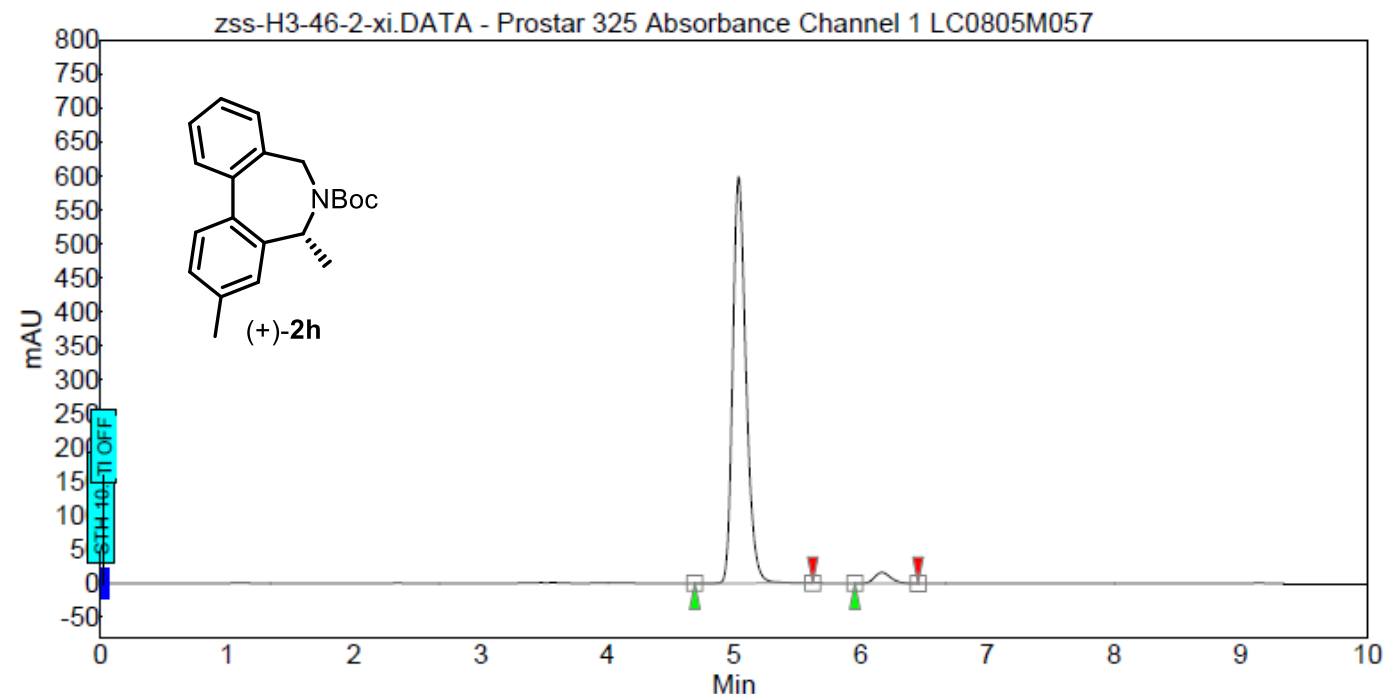

\begin{tabular}{|c|l|r|r|r|r|r|}
\hline Index & 文件名 & $\begin{array}{r}\text { 时间 } \\
\text { [Min] }\end{array}$ & $\begin{array}{r}\text { 数量 } \\
\text { [\% 面积] }\end{array}$ & $\begin{array}{r}\text { 高度 } \\
{[\text { [mAU] }}\end{array}$ & $\begin{array}{r}\text { Area } \\
\text { [mAU.Min] }\end{array}$ & $\begin{array}{r}\text { Area \% } \\
\text { [\%] }\end{array}$ \\
\hline \hline 1 & 末知 & 5.03 & 96.79 & 598.4 & 72.6 & 96.785 \\
\hline 2 & 末知 & 6.17 & 3.21 & 16.2 & 2.4 & 3.215 \\
\hline & & & & & & \\
\hline Total & & & 100.00 & 614.5 & 75.0 & 100.000 \\
\hline
\end{tabular}




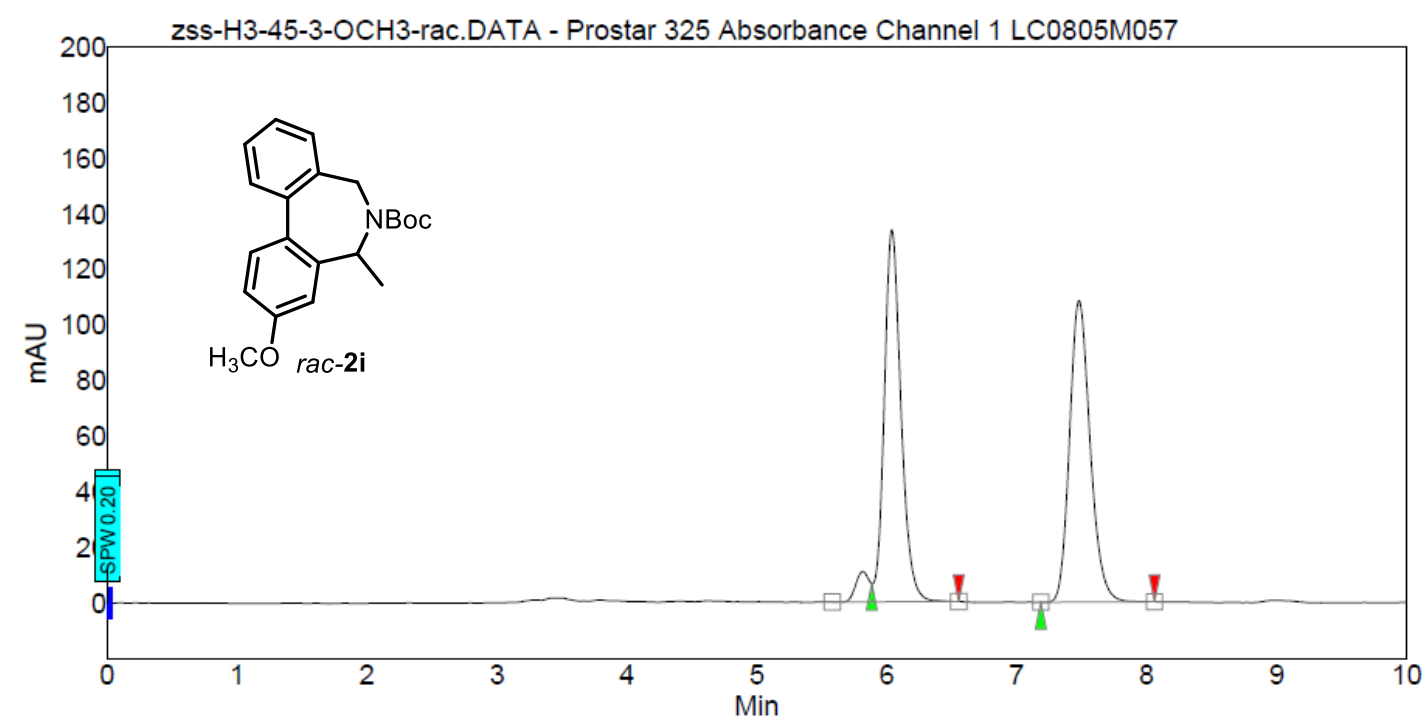

\begin{tabular}{|c|c|c|c|c|c|c|}
\hline Index & 文件名 & $\begin{array}{l}\begin{array}{l}\text { 时间 } \\
\text { [Min] }\end{array} \\
\end{array}$ & [\% 面数量 & $\begin{array}{r}\text { 高度 } \\
\text { [mAU] }\end{array}$ & $\begin{array}{r}\text { Area } \\
\text { [mAU.Min] }\end{array}$ & $\begin{array}{r}\text { Area \% } \\
{[\%]}\end{array}$ \\
\hline 2 & 本知 & 6.04 & 50.18 & 133.9 & 20.1 & 50.184 \\
\hline 1 & 末知 & 7.48 & 49.82 & 108.5 & 19.9 & 49.816 \\
\hline Total & & & 100.00 & 242.4 & 40.0 & 100.000 \\
\hline
\end{tabular}

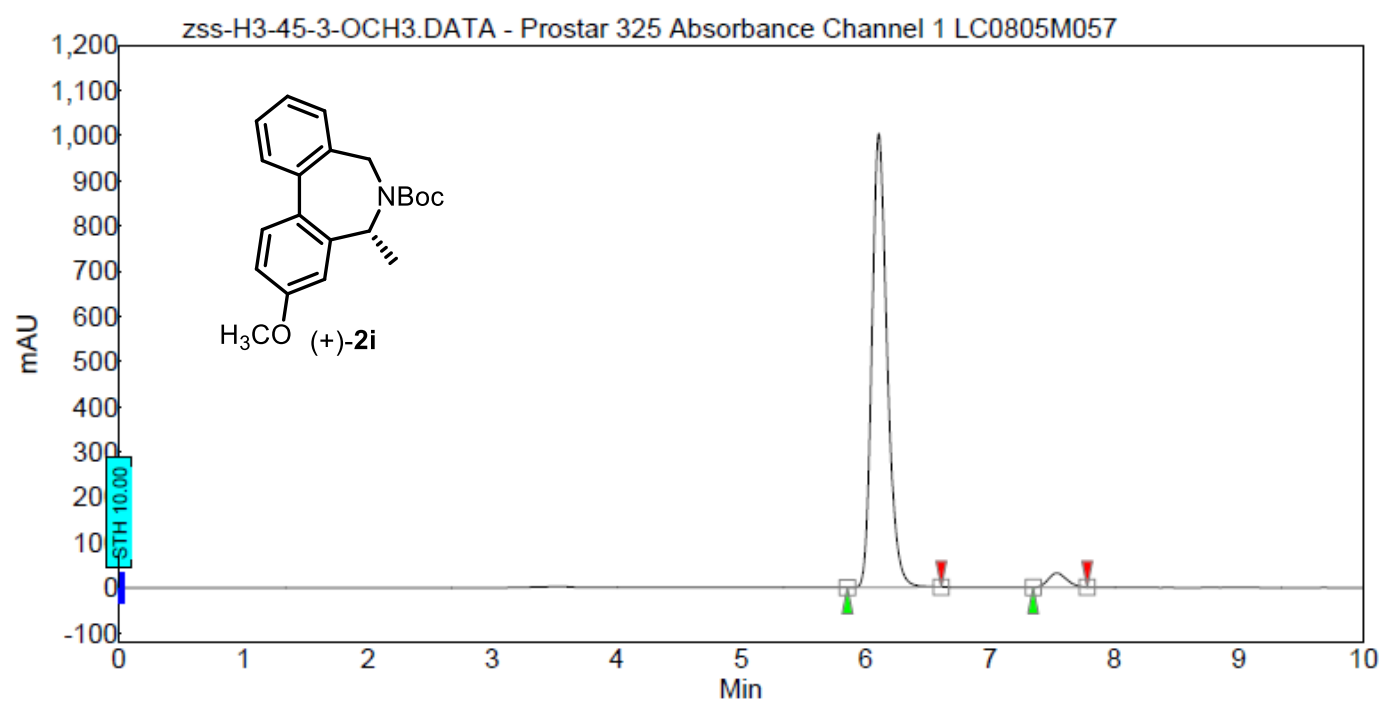

\begin{tabular}{|c|c|c|c|c|c|c|}
\hline Index & 文件名 & $\begin{array}{l}\text { 时间 } \\
\text { [Min] }\end{array}$ & [\% 面数量 & $\begin{array}{r}\text { 高度 } \\
\text { [mAU] }\end{array}$ & $\begin{array}{r}\text { Area } \\
\text { [mAU.Min] }\end{array}$ & $\begin{array}{r}\text { Area \% } \\
{[\%]} \\
\end{array}$ \\
\hline 1 & 本知 & 6.11 & 96.28 & 1003.5 & 142.5 & 96.282 \\
\hline 2 & 末知 & 7.53 & 3.72 & 31.5 & 5.5 & 3.718 \\
\hline Total & & & 100.00 & 1035.0 & 148.0 & 100.000 \\
\hline
\end{tabular}




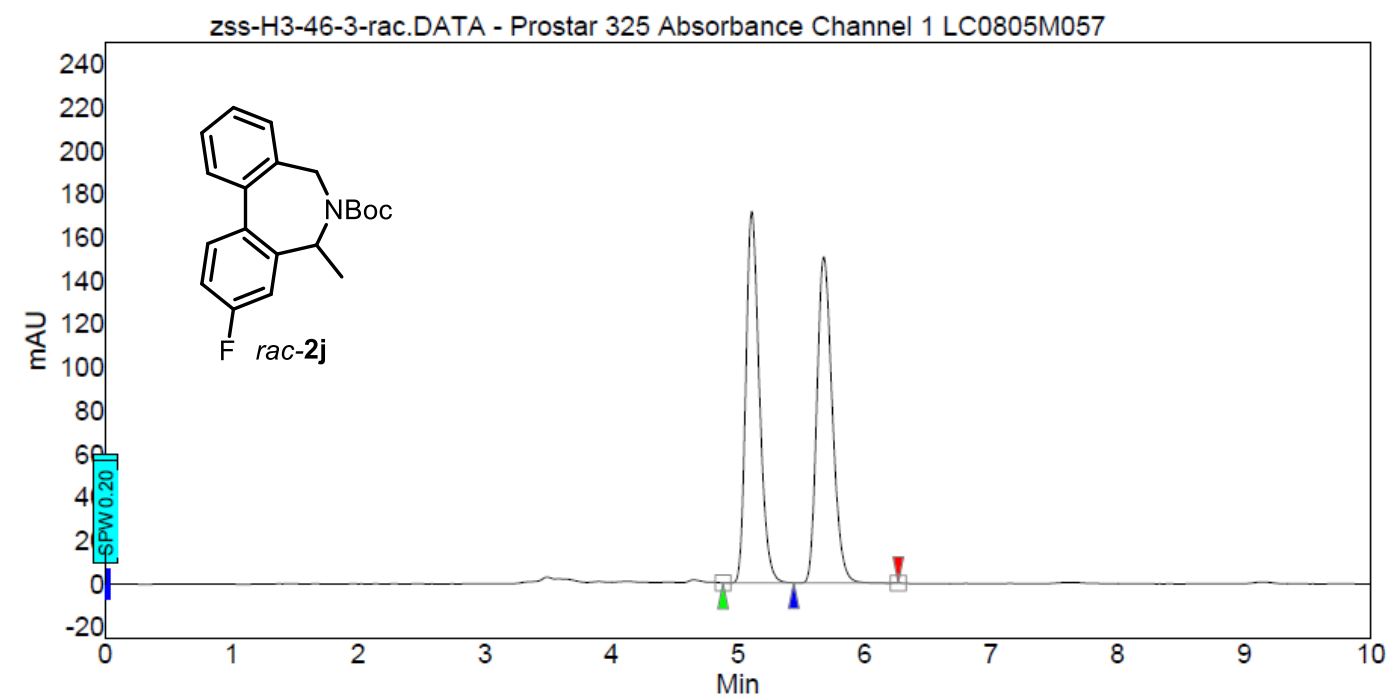

\begin{tabular}{|c|l|r|r|r|r|r|}
\hline Index & 文件名 & $\begin{array}{r}\text { 时间 } \\
\text { [Min] }\end{array}$ & $\begin{array}{r}\text { 数量 } \\
\text { [\% 面积] }\end{array}$ & $\begin{array}{r}\text { 高度 } \\
\text { [mAU] }\end{array}$ & $\begin{array}{r}\text { Area } \\
\text { [mAU.Min] }\end{array}$ & $\begin{array}{r}\text { Area \% } \\
\text { [\%] }\end{array}$ \\
\hline \hline 1 & 末知 & 5.11 & 49.89 & 171.6 & 21.2 & 49.885 \\
\hline 2 & 末知 & 5.68 & 50.11 & 150.6 & 21.3 & 50.115 \\
\hline & & & & & & \\
\hline Total & & & 100.00 & 322.3 & 42.5 & 100.000 \\
\hline
\end{tabular}

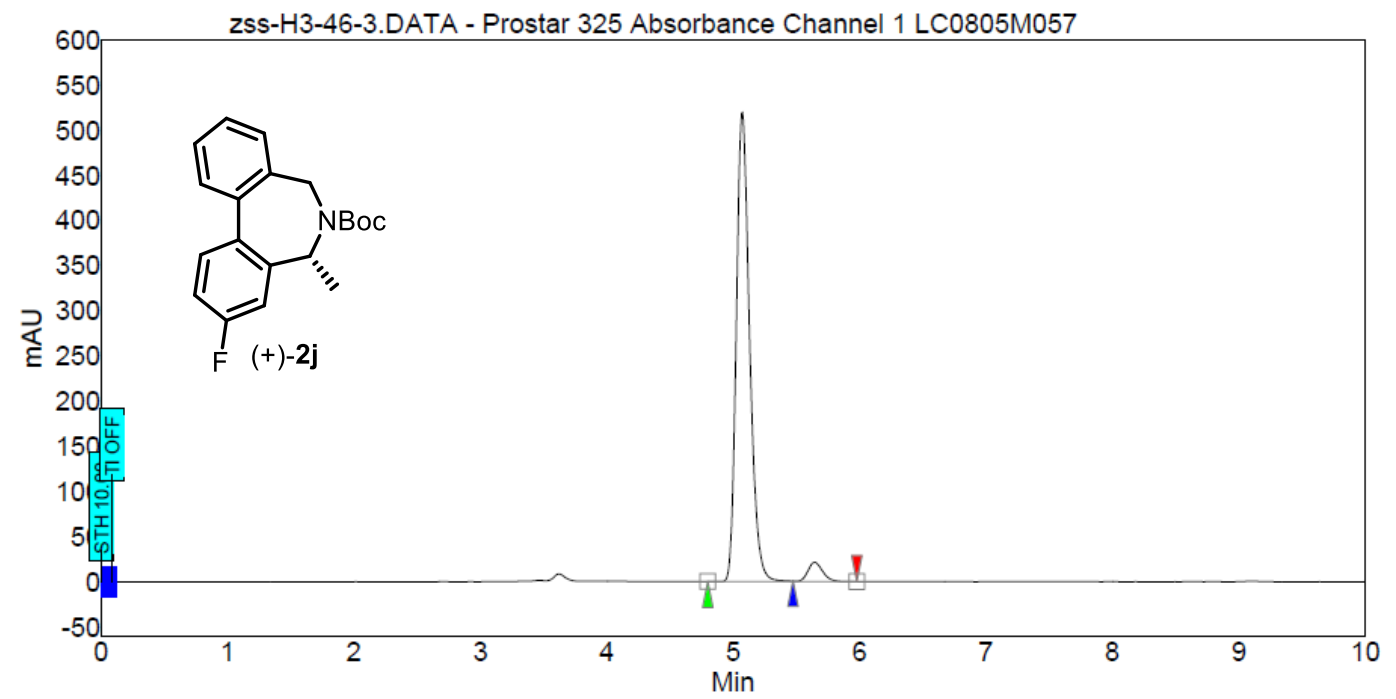

\begin{tabular}{|c|l|r|r|r|r|r|}
\hline Index & 文件名 & $\begin{array}{r}\text { 时间 } \\
\text { [Min] }\end{array}$ & $\begin{array}{r}\text { 数量 } \\
\text { [\% 面积] }\end{array}$ & $\begin{array}{r}\text { 高度 } \\
\text { [mAU] }\end{array}$ & $\begin{array}{r}\text { Area } \\
\text { [mAU.Min] }\end{array}$ & $\begin{array}{r}\text { Area \% } \\
\text { [\%] }\end{array}$ \\
\hline \hline 1 & 末知 & 5.07 & 95.62 & 520.0 & 63.3 & 95.615 \\
\hline 2 & 末知 & 5.65 & 4.38 & 21.4 & 2.9 & 4.385 \\
\hline & & & & & & \\
\hline Total & & & 100.00 & 541.3 & 66.2 & 100.000 \\
\hline
\end{tabular}




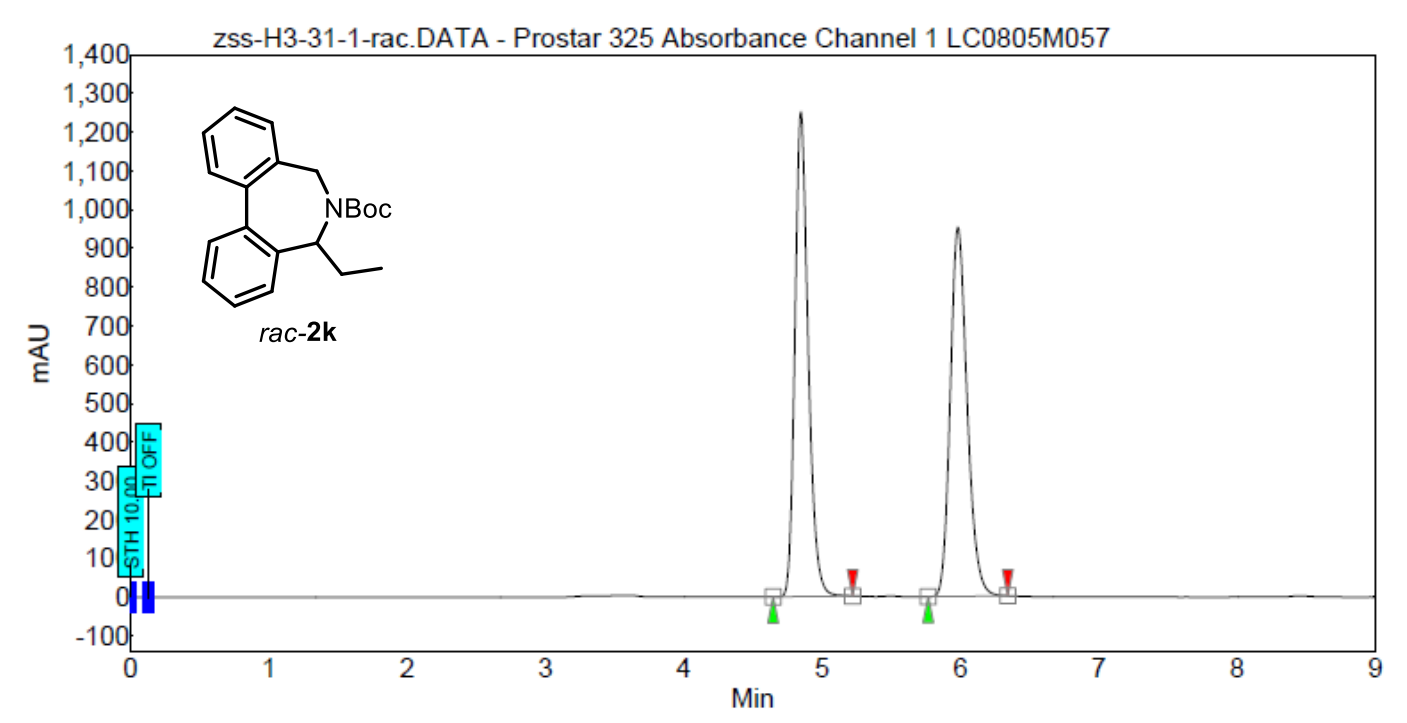

\begin{tabular}{|c|l|r|r|r|r|r|}
\hline Index & 文件名 & $\begin{array}{r}\text { 时间 } \\
\text { [Min] }\end{array}$ & $\begin{array}{r}\text { 数量 } \\
\text { [\% 面积] }\end{array}$ & $\begin{array}{r}\text { 高度 } \\
\text { [mAU] }\end{array}$ & $\begin{array}{r}\text { Area } \\
\text { [mAU.Min] }\end{array}$ & $\begin{array}{r}\text { Area \% } \\
\text { [\%] }\end{array}$ \\
\hline \hline 1 & 末知 & 4.85 & 50.70 & 1250.9 & 138.9 & 50.704 \\
\hline 2 & 末知 & 5.98 & 49.30 & 952.7 & 135.1 & 49.296 \\
\hline & & & & & & \\
\hline Total & & & 100.00 & 2203.6 & 274.0 & 100.000 \\
\hline
\end{tabular}

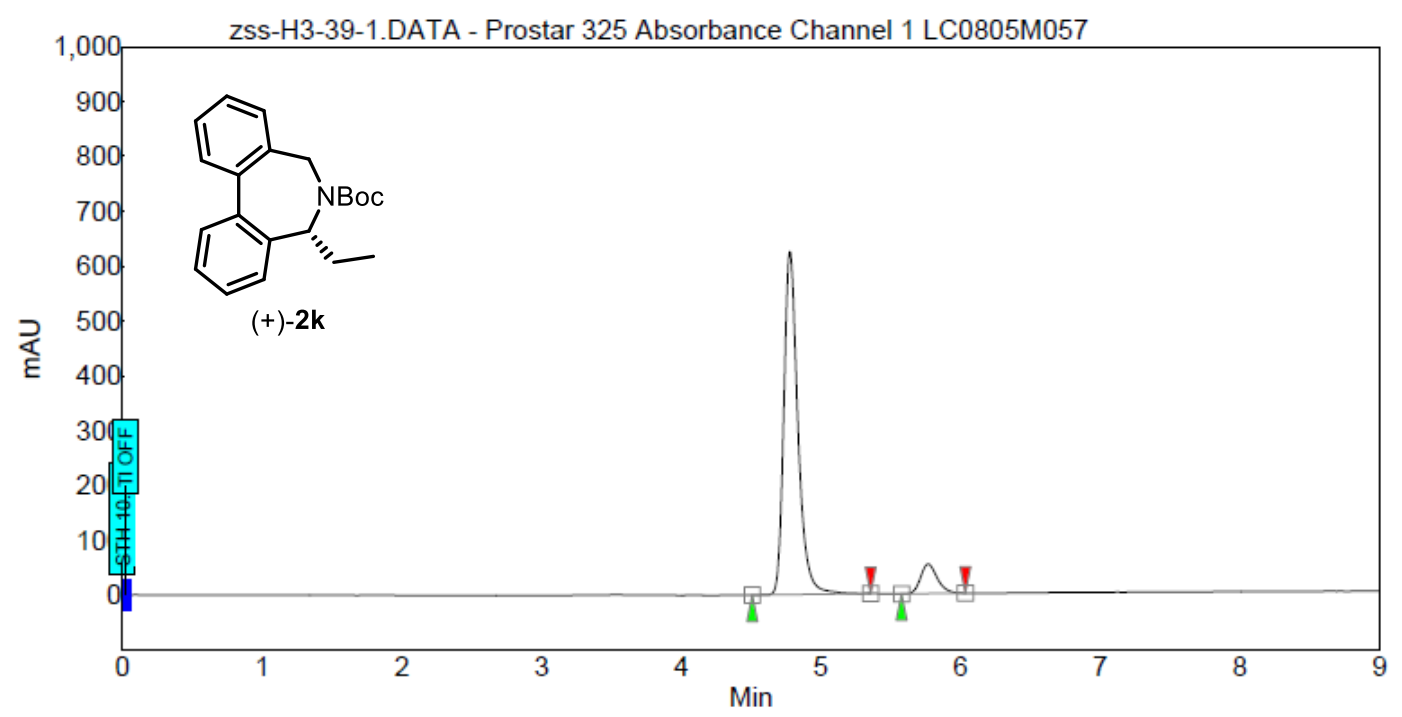

\begin{tabular}{|c|c|c|c|c|c|c|}
\hline Index & 文件名 & $\begin{array}{r}\text { 时间 } \\
\text { [Min] }\end{array}$ & [\% 面数量 & $\begin{array}{r}\text { 高度 } \\
\text { [mAU] }\end{array}$ & $\begin{array}{r}\text { Area } \\
\text { [mAU.Min] }\end{array}$ & $\begin{array}{r}\text { Area \% } \\
{[\%]} \\
\end{array}$ \\
\hline 1 & 末知 & 4.77 & 90.74 & 625.2 & 72.9 & 90.742 \\
\hline 2 & 本知 & 5.77 & 9.26 & 53.8 & 7.4 & 9.258 \\
\hline Total & & & 100.00 & 679.0 & 80.4 & 100.000 \\
\hline
\end{tabular}




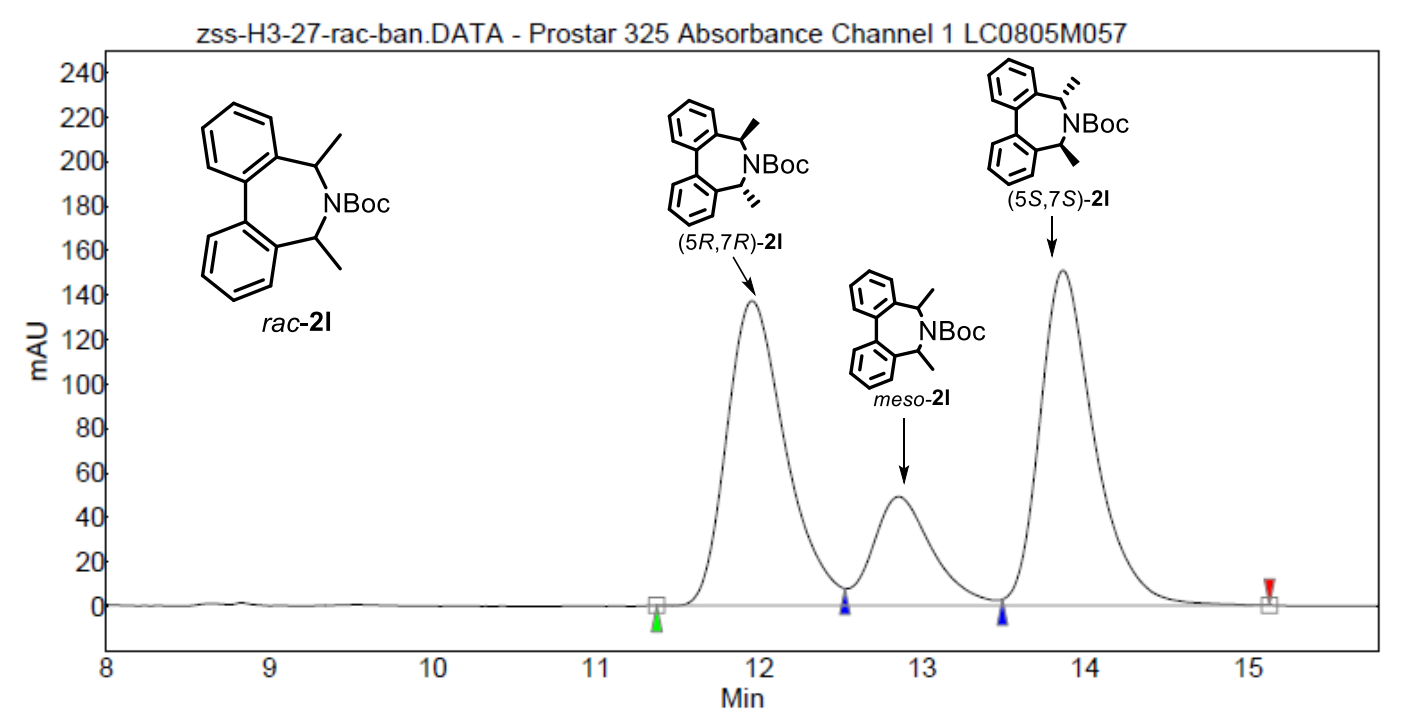

\begin{tabular}{|c|l|r|r|r|r|r|}
\hline Index & 文件名 & $\begin{array}{r}\text { 时间 } \\
\text { [Min] }\end{array}$ & $\begin{array}{r}\text { 数量 } \\
\text { [\% 面积] }\end{array}$ & \multicolumn{1}{|c|}{ [高度 } & $\begin{array}{r}\text { Area } \\
\text { [mAU.Min] }\end{array}$ & $\begin{array}{r}\text { Area \% } \\
\text { [\%] }\end{array}$ \\
\hline \hline 1 & 末知 & 11.96 & 41.99 & 136.8 & 57.2 & 41.994 \\
\hline 2 & 末知 & 12.85 & 15.20 & 48.9 & 20.7 & 15.201 \\
\hline 3 & 末知 & 13.87 & 42.80 & 150.5 & 58.3 & 42.805 \\
\hline & & & & & & \\
\hline Total & & & 100.00 & 336.2 & 136.2 & 100.000 \\
\hline
\end{tabular}

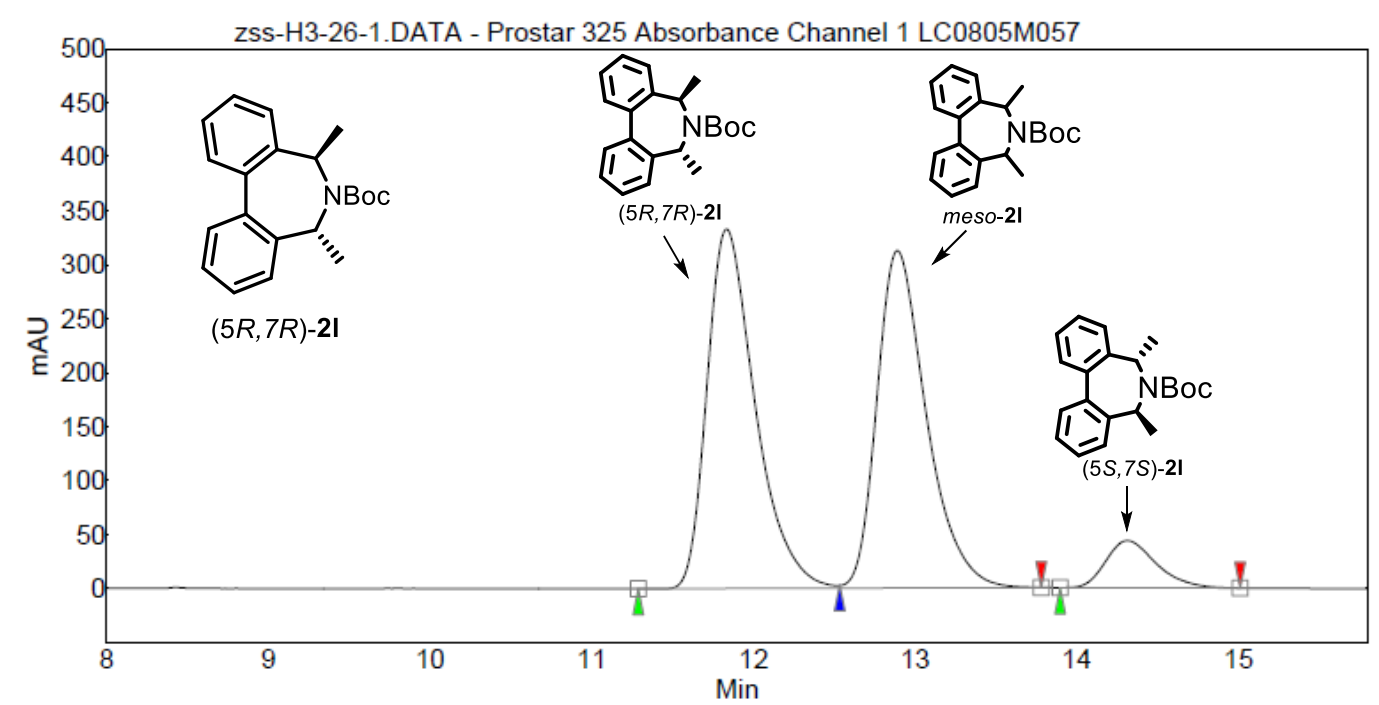

\begin{tabular}{|c|l|r|r|r|r|r|}
\hline Index & 文件名 & $\begin{array}{r}\text { 时间 } \\
\text { [Min] }\end{array}$ & $\begin{array}{r}\text { 数量 } \\
{[\% \text { 面积] }}\end{array}$ & \multicolumn{1}{|c|}{$\begin{array}{r}\text { 高度 } \\
\text { [mAU] }\end{array}$} & $\begin{array}{r}\text { Area } \\
\text { [mAU.Min] }\end{array}$ & $\begin{array}{r}\text { Area \% } \\
{[\%]}\end{array}$ \\
\hline \hline 1 & 末知 & 11.83 & 47.61 & 332.9 & 114.9 & 47.605 \\
\hline 3 & 末知 & 12.89 & 45.85 & 312.4 & 110.6 & 45.850 \\
\hline 2 & 末知 & 14.31 & 6.54 & 43.8 & 15.8 & 6.545 \\
\hline & & & & & & \\
\hline Total & & & 100.00 & 689.0 & 241.3 & 100.000 \\
\hline
\end{tabular}




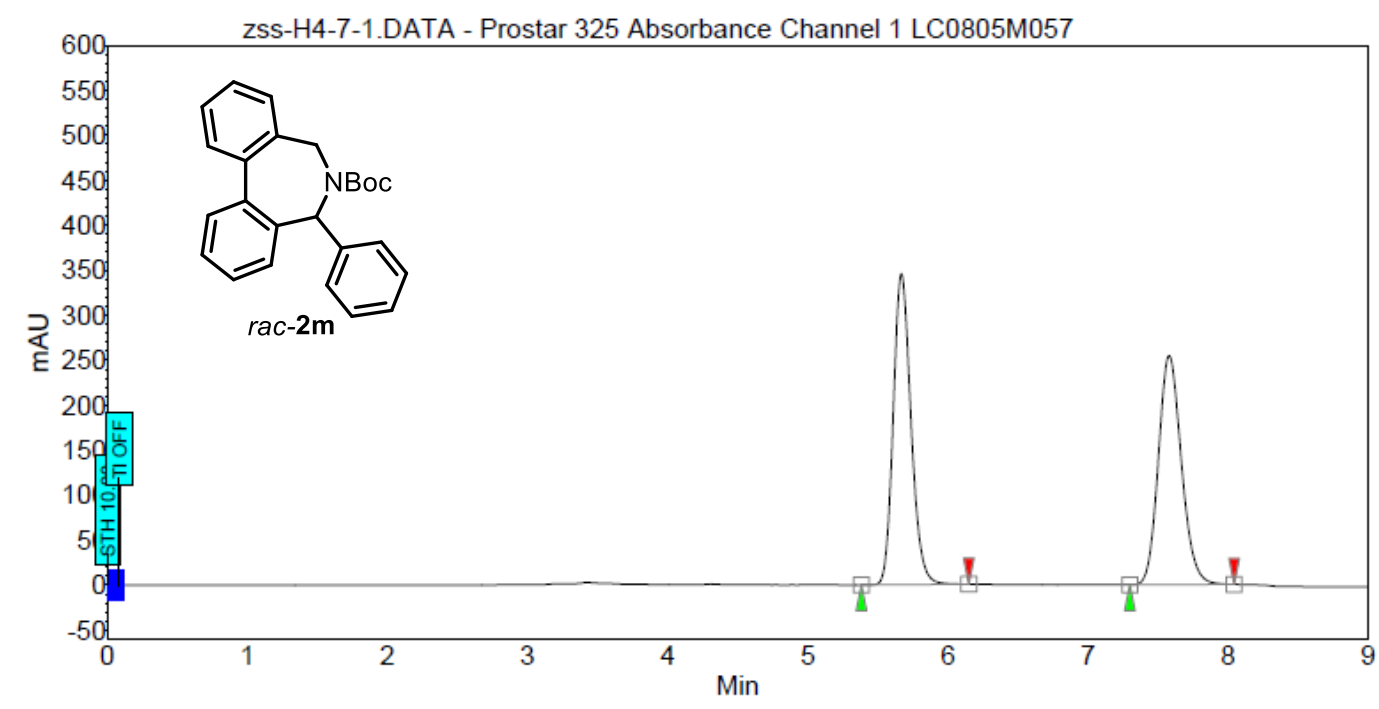

\begin{tabular}{|c|l|r|r|r|r|r|}
\hline \multicolumn{2}{|l|}{ Index 文件名 } & \multicolumn{1}{|c|}{$\begin{array}{l}\text { 时间 } \\
\text { [Min] }\end{array}$} & $\begin{array}{r}\text { 数量 } \\
\text { [\% 面积] }\end{array}$ & $\begin{array}{r}\text { 高度 } \\
\text { [mAU] }\end{array}$ & $\begin{array}{r}\text { Area } \\
\text { [mAU.Min] }\end{array}$ & $\begin{array}{r}\text { Area \% } \\
\text { [\%] }\end{array}$ \\
\hline \hline 1 & 末知 & 5.67 & 51.07 & 345.7 & 50.7 & 51.068 \\
\hline 2 & 末知 & 7.58 & 48.93 & 254.6 & 48.6 & 48.932 \\
\hline & & & & & & \\
\hline Total & & & 100.00 & 600.3 & 99.2 & 100.000 \\
\hline
\end{tabular}

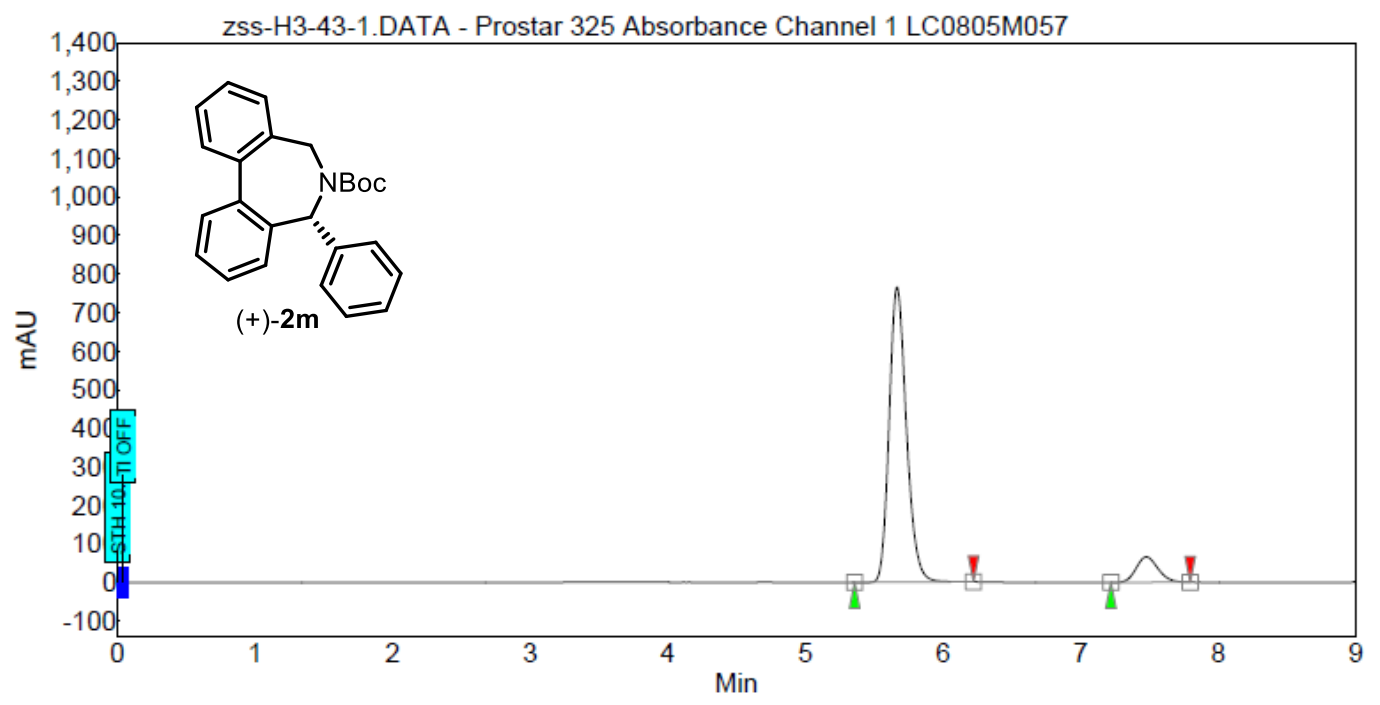

\begin{tabular}{|c|l|r|r|r|r|r|}
\hline Index & 文件名 & $\begin{array}{r}\text { 时间 } \\
{[\mathrm{Min}]}\end{array}$ & $\begin{array}{r}\text { 数量 } \\
\text { [\% 面积] }\end{array}$ & $\begin{array}{r}\text { 高度 } \\
{[\mathrm{mAU}]}\end{array}$ & $\begin{array}{r}\text { Area } \\
\text { [mAU.Min] }\end{array}$ & $\begin{array}{r}\text { Area \% } \\
\text { [\%] }\end{array}$ \\
\hline \hline 1 & 末知 & 5.67 & 90.12 & 765.4 & 111.3 & 90.116 \\
\hline 2 & 末知 & 7.48 & 9.88 & 66.0 & 12.2 & 9.884 \\
\hline & & & & & & \\
\hline Total & & & 100.00 & 831.4 & 123.5 & 100.000 \\
\hline
\end{tabular}




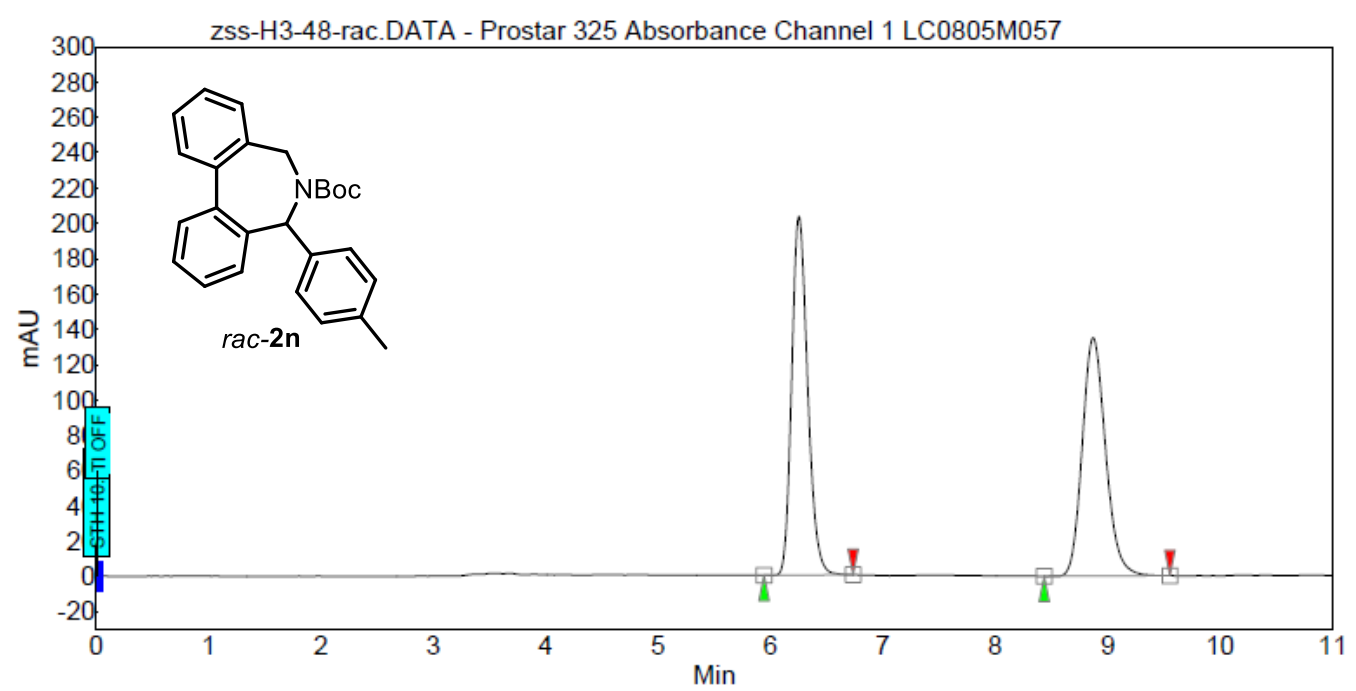

\begin{tabular}{|c|lr|r|r|r|r|}
\hline Index & 文件名 & $\begin{array}{r}\text { 时间 } \\
\text { [Min] }\end{array}$ & $\begin{array}{r}\text { 数量 } \\
\text { [\% 面积] }\end{array}$ & \multicolumn{1}{|c|}{$\begin{array}{r}\text { 高度 } \\
\text { [mAU] }\end{array}$} & $\begin{array}{r}\text { Area } \\
\text { [mAU.Min] }\end{array}$ & $\begin{array}{r}\text { Area \% } \\
\text { [\%] }\end{array}$ \\
\hline \hline 1 & 末知 & 6.25 & 50.73 & 203.1 & 33.4 & 50.727 \\
\hline 2 & 末知 & 8.87 & 49.27 & 135.0 & 32.4 & 49.273 \\
\hline & & & & & & \\
\hline Total & & & 100.00 & 338.1 & 65.8 & 100.000 \\
\hline
\end{tabular}

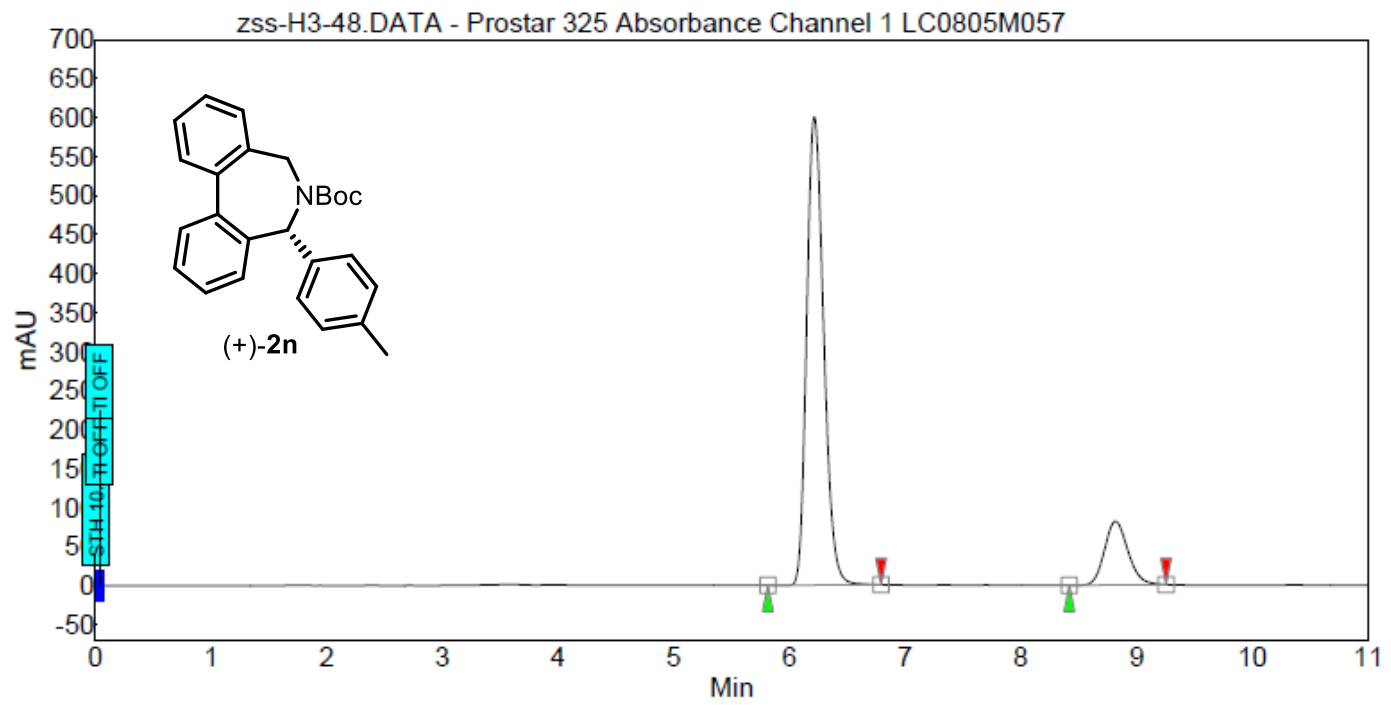

\begin{tabular}{|c|c|c|c|c|c|c|}
\hline Index & 文件名 & $\begin{array}{l}\text { 时间 } \\
\text { [Min] }\end{array}$ & [\% 面数量 & $\begin{array}{r}\text { 高度 } \\
\text { [mAU] }\end{array}$ & $\begin{array}{r}\text { Area } \\
\text { [mAU.Min] }\end{array}$ & $\begin{array}{r}\text { Area \% } \\
{[\%]}\end{array}$ \\
\hline 1 & 本知 & 6.21 & 84.22 & 600.4 & 102.1 & 84.219 \\
\hline 2 & 末知 & 8.81 & 15.78 & 81.5 & 19.1 & 15.781 \\
\hline Total & & & 100.00 & 681.9 & 121.3 & 100.000 \\
\hline
\end{tabular}




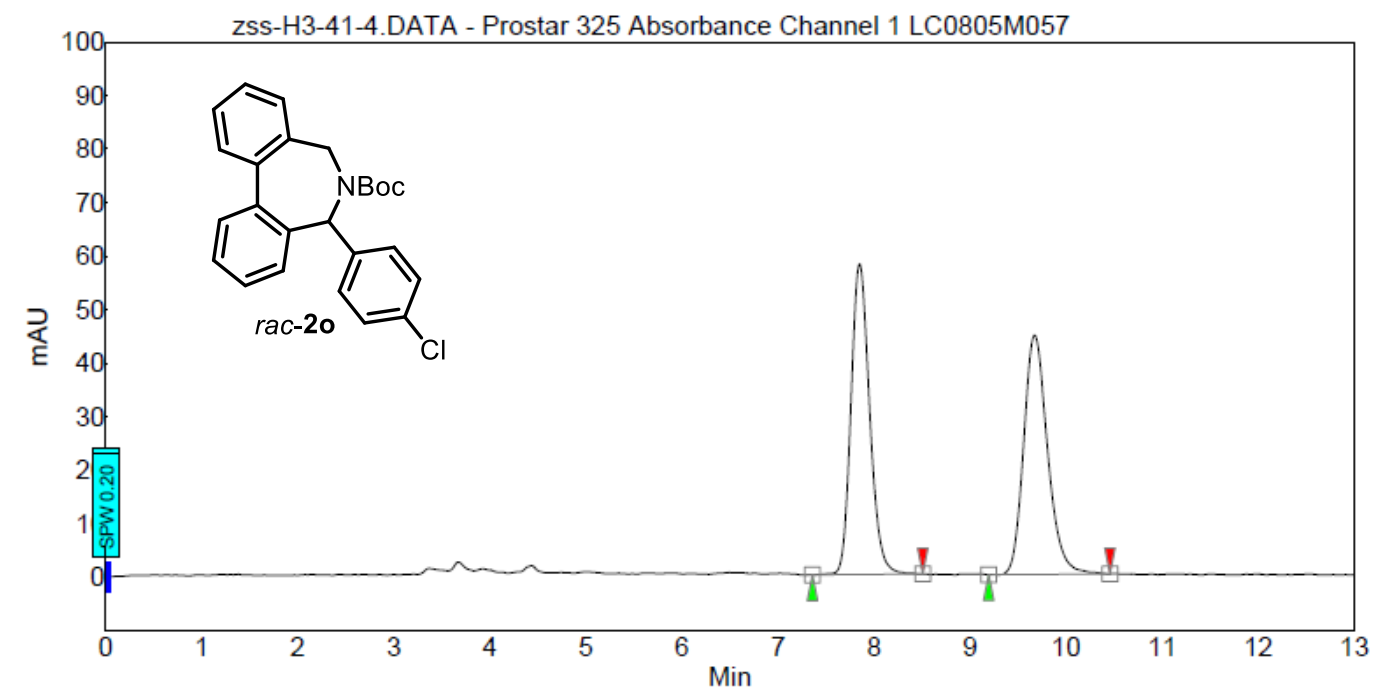

\begin{tabular}{|c|l|r|r|r|r|r|}
\hline Index & 文件名 & $\begin{array}{r}\text { 时间 } \\
\text { [Min] }\end{array}$ & $\begin{array}{r}\text { 数量 } \\
\text { [\% 面积] }\end{array}$ & $\begin{array}{r}\text { 高度 } \\
\text { [mAU] }\end{array}$ & $\begin{array}{r}\text { Area } \\
\text { [mAU.Min] }\end{array}$ & $\begin{array}{r}\text { Area \% } \\
\text { [\%] }\end{array}$ \\
\hline \hline 1 & 末知 & 7.85 & 50.30 & 58.0 & 13.1 & 50.296 \\
\hline 2 & 末知 & 9.67 & 49.70 & 44.7 & 12.9 & 49.704 \\
\hline & & & & & & \\
\hline Total & & & 100.00 & 102.7 & 26.0 & 100.000 \\
\hline
\end{tabular}

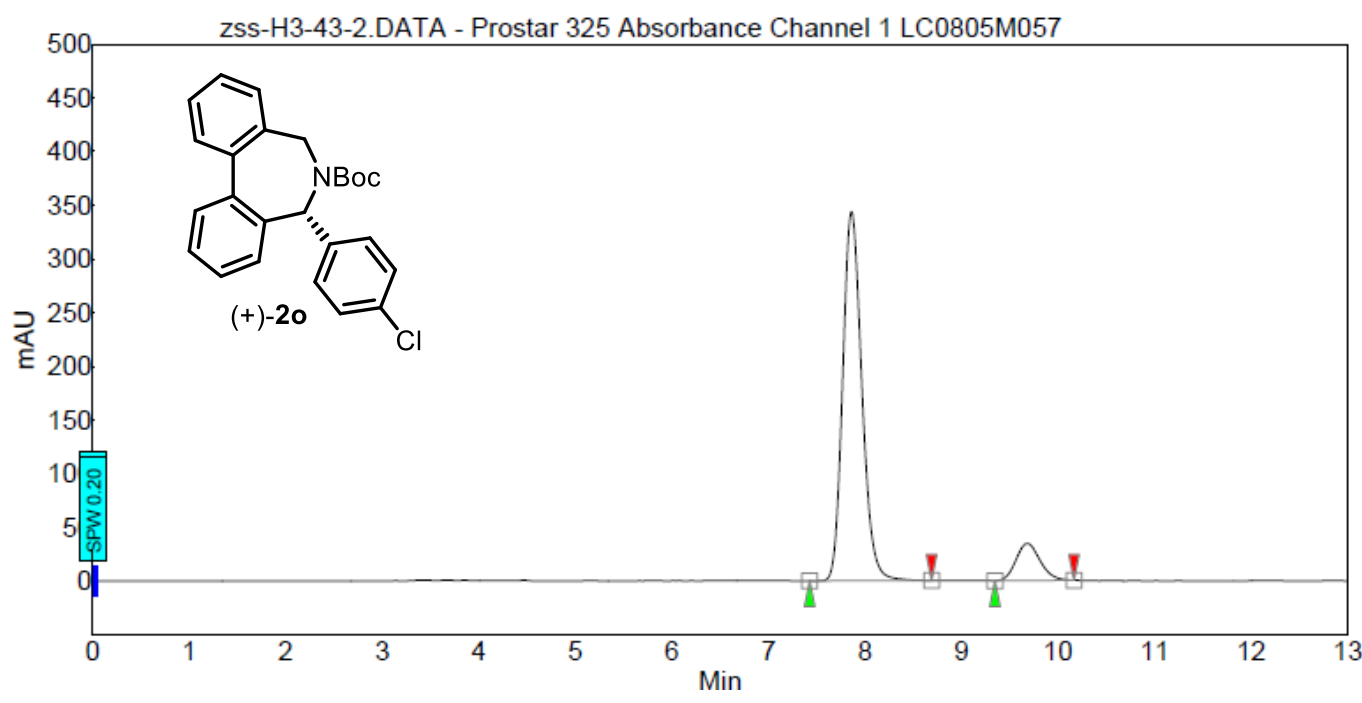

\begin{tabular}{|c|c|c|c|c|c|c|}
\hline Index & 文件名 & $\begin{array}{r}\text { 时间 } \\
\text { [Min] }\end{array}$ & [\% 面积] & 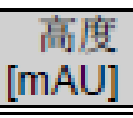 & $\begin{array}{r}\text { Area } \\
\text { [mAU.Min] } \\
\end{array}$ & $\begin{array}{r}\text { Area } \% \\
{[\%]} \\
\end{array}$ \\
\hline 1 & 本知 & 7.86 & 88.72 & 344.0 & 78.8 & 88.716 \\
\hline 2 & 本知 & 9.68 & 11.28 & 34.6 & 10.0 & 11.284 \\
\hline Total & & & 100.00 & 378.6 & 88.8 & 100.000 \\
\hline
\end{tabular}




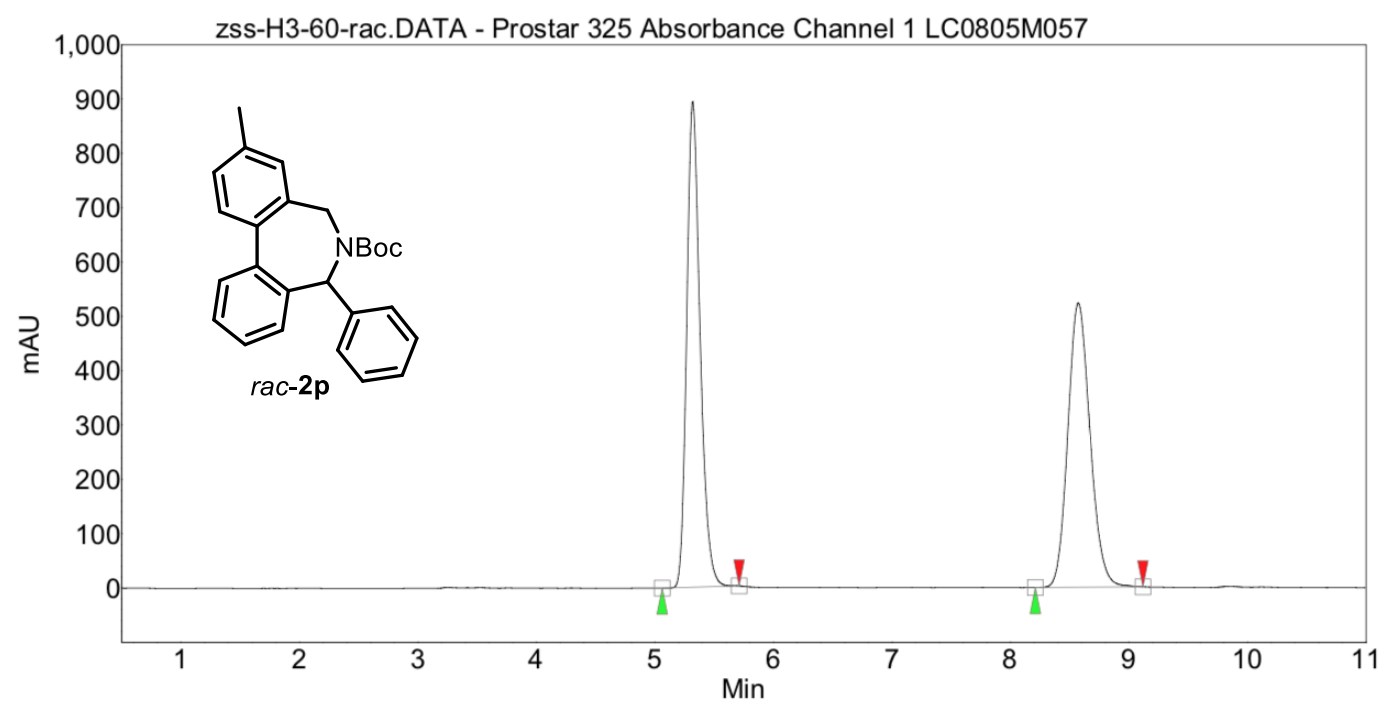

\begin{tabular}{|c|l|r|r|r|r|r|}
\hline Index & 文件名 & $\begin{array}{r}\text { 时间 } \\
{[\text { Min] }}\end{array}$ & $\begin{array}{r}\text { 数量 } \\
{[\% \text { 面积] }}\end{array}$ & $\begin{array}{r}\text { 高度 } \\
{[\mathrm{mAU}]}\end{array}$ & $\begin{array}{r}\text { Area } \\
\text { [mAU.Min] }\end{array}$ & $\begin{array}{r}\text { Area \% } \\
{[\%]}\end{array}$ \\
\hline \hline 1 & 未知 & 5.32 & 50.73 & 893.8 & 117.0 & 50.727 \\
\hline 2 & 未知 & 8.57 & 49.27 & 523.3 & 113.7 & 49.273 \\
\hline & & & & & & \\
\hline Total & & & 100.00 & 1417.1 & 230.7 & 100.000 \\
\hline
\end{tabular}

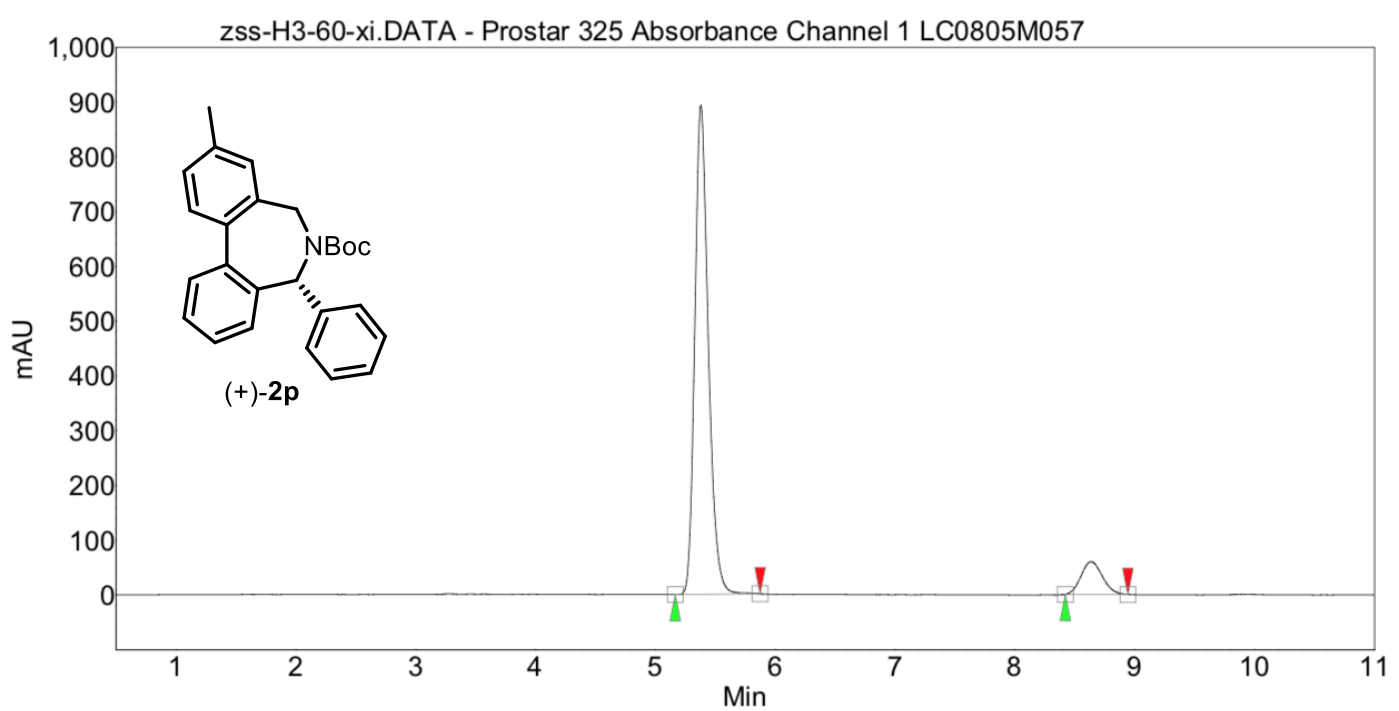

\begin{tabular}{|c|l|r|r|r|r|r|}
\hline Index & 文件名 & $\begin{array}{r}\text { 时间 } \\
\text { [Min] }\end{array}$ & $\begin{array}{r}\text { 数量 } \\
\text { [\% 面积] }\end{array}$ & $\begin{array}{r}\text { 高度 } \\
{[\mathrm{mAU}]}\end{array}$ & $\begin{array}{r}\text { Area } \\
\text { [mAU.Min] }\end{array}$ & $\begin{array}{r}\text { Area \% } \\
{[\%]}\end{array}$ \\
\hline \hline 2 & 未知 & 5.38 & 90.44 & 893.7 & 116.8 & 90.441 \\
\hline 1 & 未知 & 8.63 & 9.56 & 59.7 & 12.3 & 9.559 \\
\hline & & & & & & \\
\hline Total & & & 100.00 & 953.4 & 129.1 & 100.000 \\
\hline
\end{tabular}




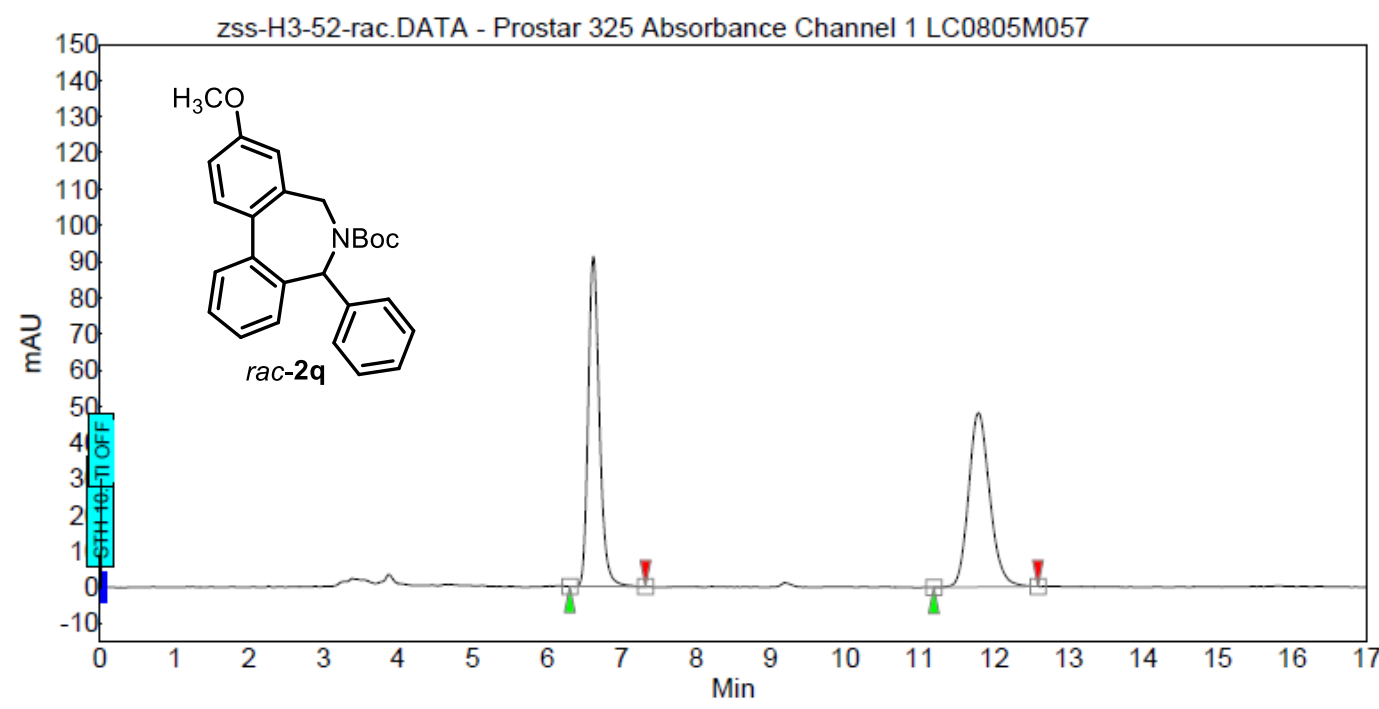

\begin{tabular}{|c|l|r|r|r|r|r|}
\hline Index & 文件名 & $\begin{array}{r}\text { 时间 } \\
{[\mathrm{Min}]}\end{array}$ & $\begin{array}{r}\text { 数量 } \\
\text { [\% 面积] }\end{array}$ & $\begin{array}{r}\text { 高度 } \\
\text { [mAU] }\end{array}$ & $\begin{array}{r}\text { Area } \\
\text { [mAU.Min] }\end{array}$ & $\begin{array}{r}\text { Area \% } \\
\text { [\%] }\end{array}$ \\
\hline \hline 1 & 末知 & 6.62 & 50.14 & 91.2 & 16.0 & 50.144 \\
\hline 2 & 末知 & 11.79 & 49.86 & 48.1 & 15.9 & 49.856 \\
\hline & & & & & & \\
\hline Total & & & 100.00 & 139.3 & 31.8 & 100.000 \\
\hline
\end{tabular}

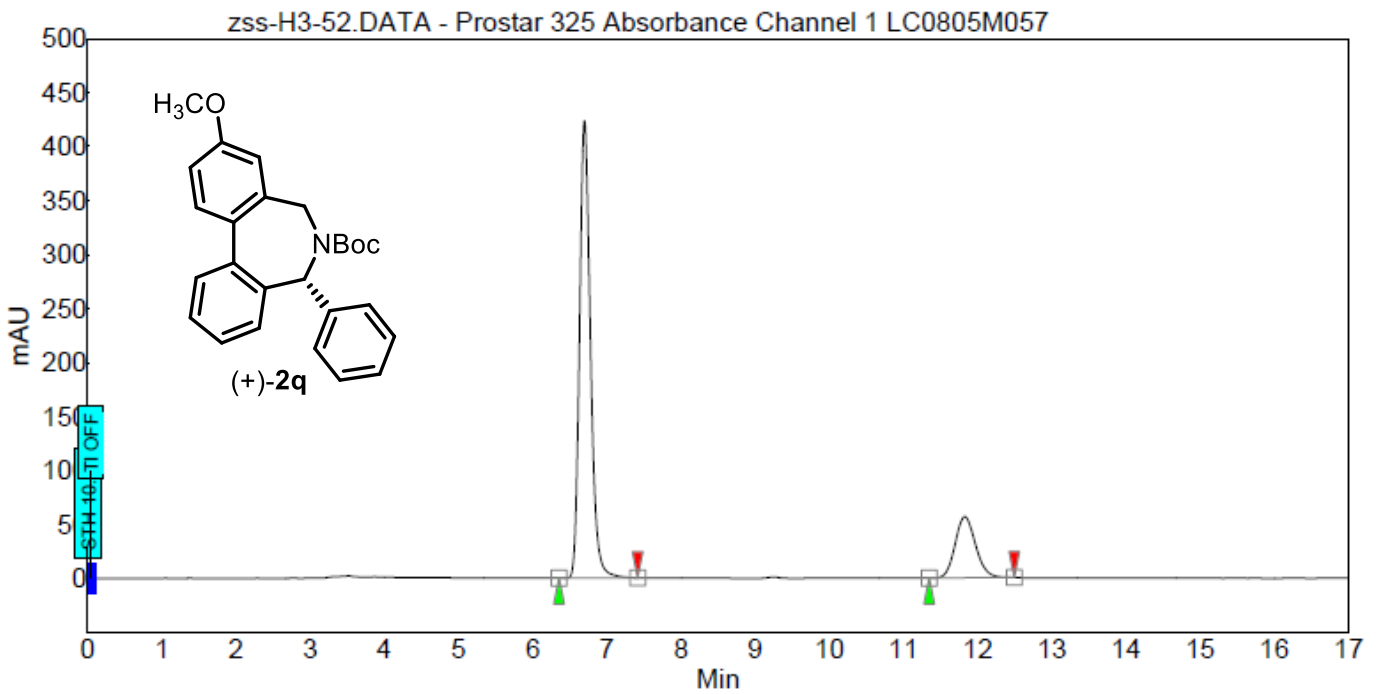

\begin{tabular}{|c|l|r|r|r|r|r|}
\hline Index & 文件名 & $\begin{array}{r}\text { 时间 } \\
\text { [Min] }\end{array}$ & $\begin{array}{r}\text { 数量 } \\
\text { [\% 面积] }\end{array}$ & \multicolumn{1}{|c|}{$\begin{array}{r}\text { 高度 } \\
\text { [mAU] }\end{array}$} & $\begin{array}{r}\text { Area } \\
\text { [mAU.Min] }\end{array}$ & $\begin{array}{r}\text { Area \% } \\
\text { [\%] }\end{array}$ \\
\hline \hline 1 & 末知 & 6.69 & 79.88 & 423.3 & 72.2 & 79.878 \\
\hline 2 & 末知 & 11.83 & 20.12 & 56.8 & 18.2 & 20.122 \\
\hline & & & & & & \\
\hline Total & & & 100.00 & 480.2 & 90.4 & 100.000 \\
\hline
\end{tabular}




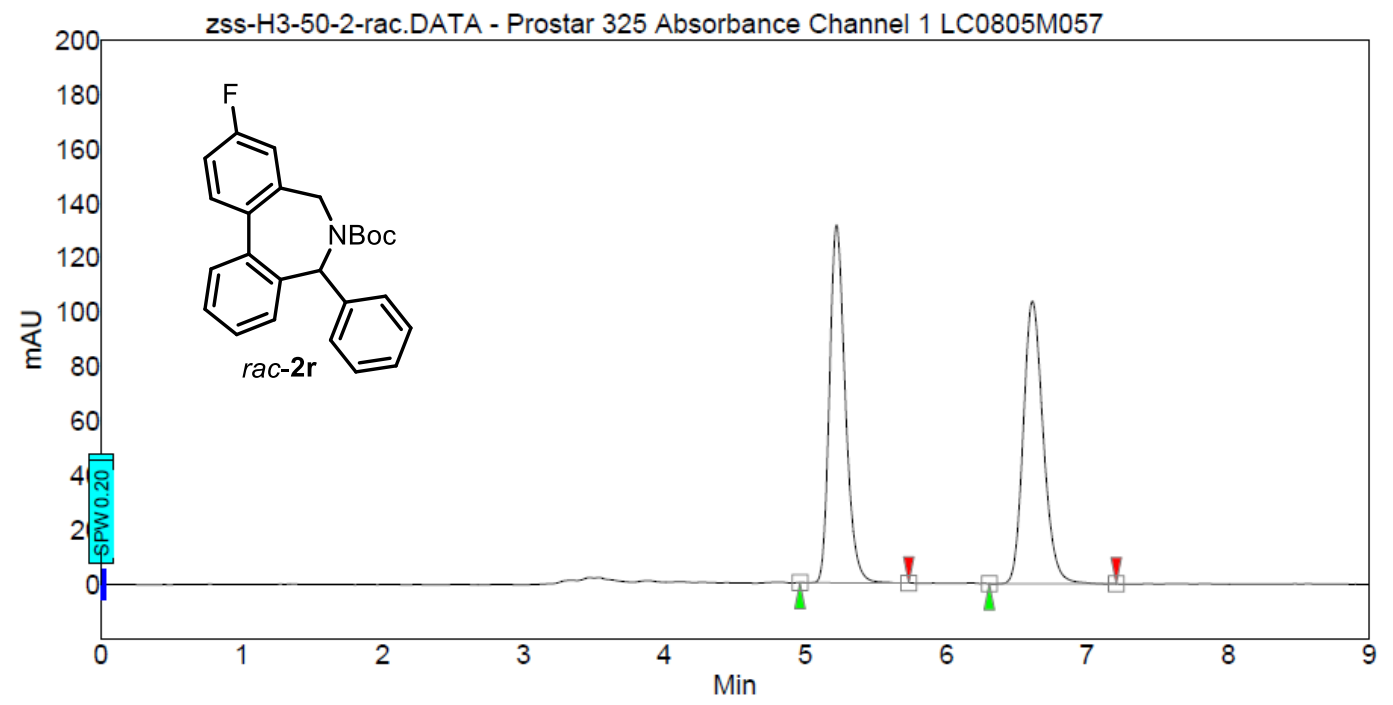

\begin{tabular}{|c|l|r|r|r|r|r|}
\hline Index & 文件名 & $\begin{array}{r}\text { 时间 } \\
{[\mathrm{Min}]}\end{array}$ & $\begin{array}{r}\text { 数量 } \\
\text { [\% 面积] }\end{array}$ & $\begin{array}{r}\text { 高度 } \\
{[\mathrm{mAU}]}\end{array}$ & $\begin{array}{r}\text { Area } \\
\text { [mAU.Min] }\end{array}$ & $\begin{array}{r}\text { Area \% } \\
\text { [\%] }\end{array}$ \\
\hline \hline 1 & 末知 & 5.22 & 50.02 & 131.6 & 17.1 & 50.024 \\
\hline 2 & 末知 & 6.61 & 49.98 & 103.9 & 17.1 & 49.976 \\
\hline & & & & & & \\
\hline Total & & & 100.00 & 235.5 & 34.1 & 100.000 \\
\hline
\end{tabular}

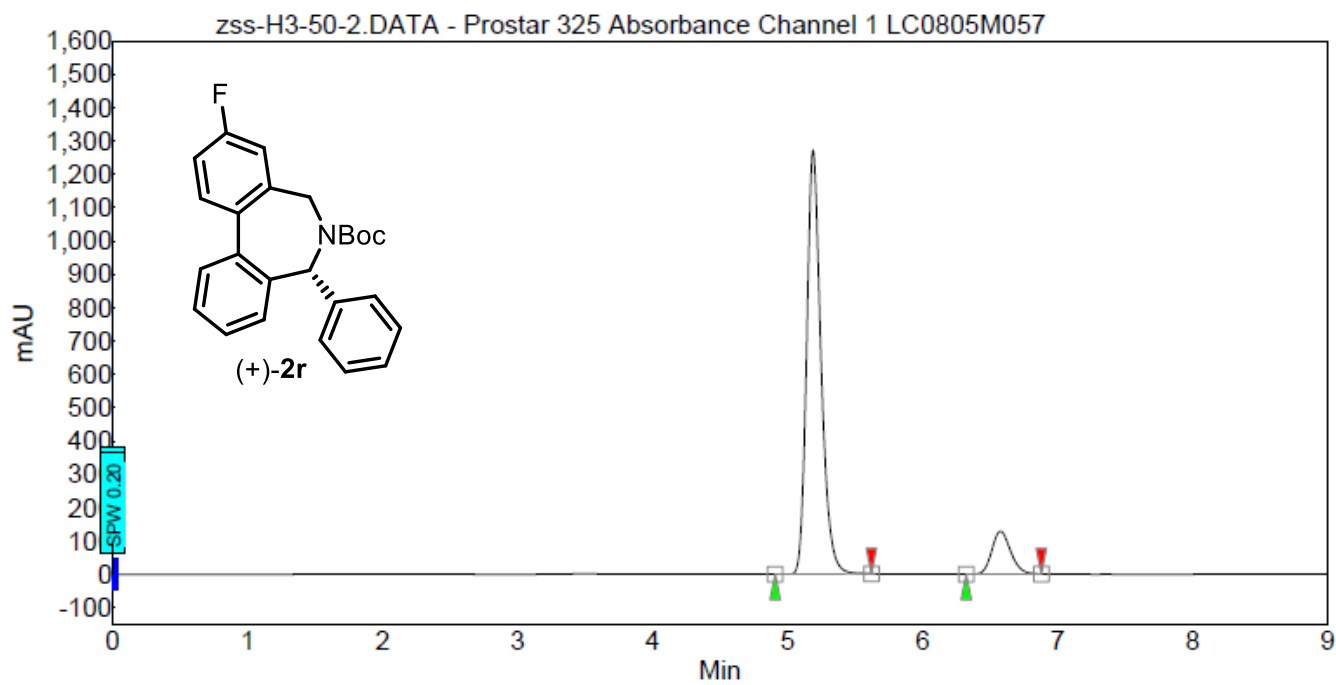

\begin{tabular}{|c|c|c|c|c|c|c|}
\hline Index & 文件名 & $\begin{array}{r}\begin{array}{r}\text { 时间 } \\
\text { [Min] }\end{array} \\
\end{array}$ & $\begin{array}{r}\text { 数量 } \\
\text { [\% 面积] } \\
\end{array}$ & $\begin{array}{r}\text { 高度 } \\
\text { [mAU] }\end{array}$ & $\begin{array}{r}\text { Area } \\
\text { [mAU.Min] }\end{array}$ & $\begin{array}{r}\text { Area \% } \\
{[\%]} \\
\end{array}$ \\
\hline 1 & 本知 & 5.19 & 88.27 & 1271.3 & 156.3 & 88.267 \\
\hline 2 & 末知 & 6.57 & 11.73 & 128.6 & 20.8 & 11.733 \\
\hline Total & & & 100.00 & 1399.8 & 177.1 & 100.000 \\
\hline
\end{tabular}




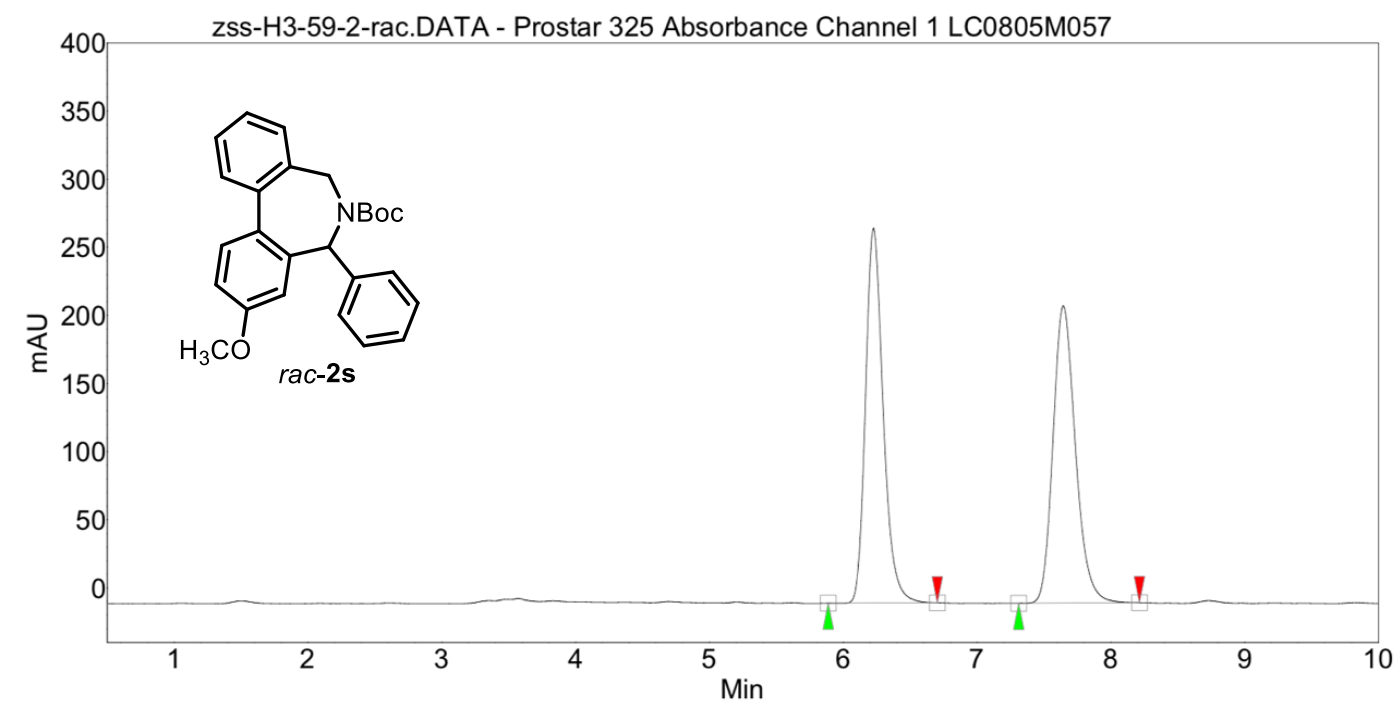

\begin{tabular}{|c|l|r|r|r|r|r|}
\hline Index & 文件名 & $\begin{array}{r}\text { 时间 } \\
\text { [Min] }\end{array}$ & $\begin{array}{r}\text { 数量 } \\
\text { [\% 面积] }\end{array}$ & $\begin{array}{r}\text { 高度 } \\
\text { [mAU] }\end{array}$ & $\begin{array}{r}\text { Area } \\
\text { [mAU.Min] }\end{array}$ & $\begin{array}{r}\text { Area \% } \\
{[\%]}\end{array}$ \\
\hline \hline 1 & 未知 & 6.23 & 49.91 & 275.3 & 42.2 & 49.909 \\
\hline 2 & 未知 & 7.65 & 50.09 & 218.1 & 42.3 & 50.091 \\
\hline & & & & & & \\
\hline Total & & & 100.00 & 493.4 & 84.5 & 100.000 \\
\hline
\end{tabular}

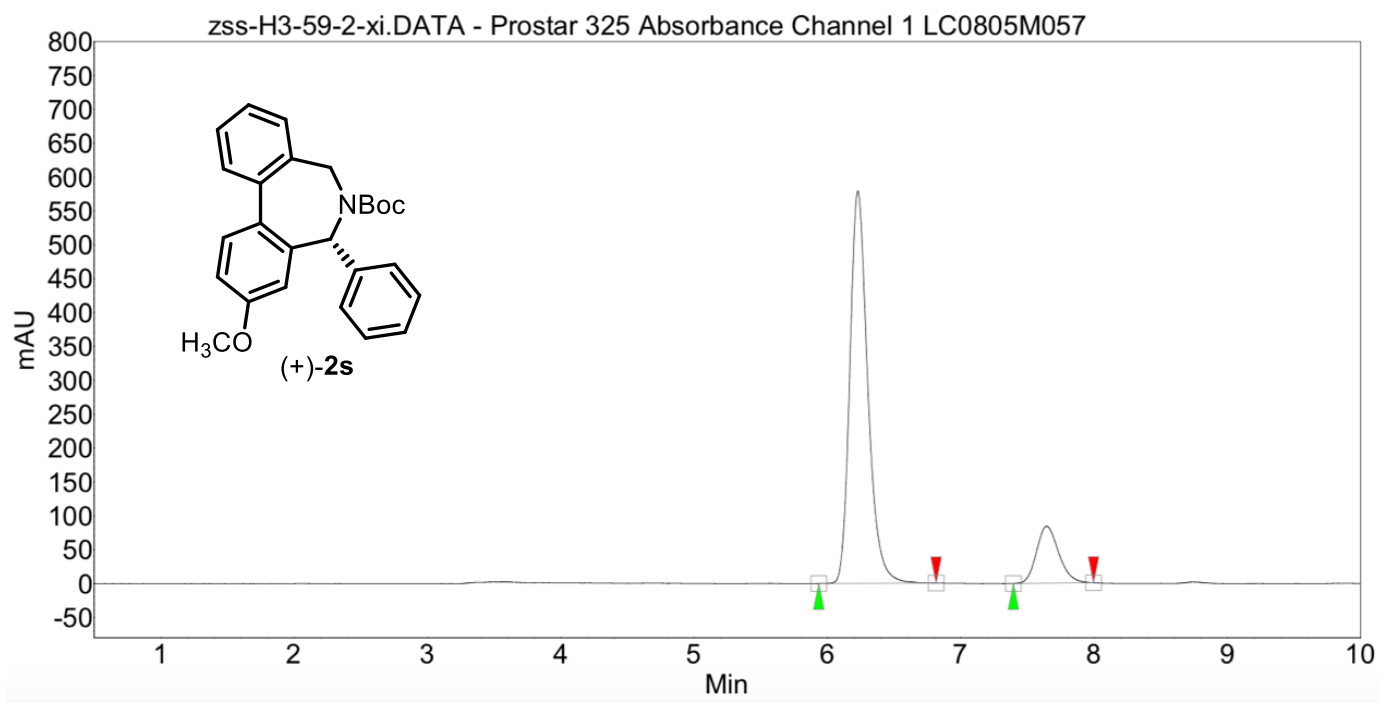

\begin{tabular}{|c|l|r|r|r|r|r|}
\hline Index & 文件名 & $\begin{array}{r}\text { 时间 } \\
\text { [Min] }\end{array}$ & $\begin{array}{r}\text { 数量 } \\
\text { [\% 面积] }\end{array}$ & $\begin{array}{r}\text { 高度 } \\
\text { [mAU] }\end{array}$ & $\begin{array}{r}\text { Area } \\
\text { [mAU.Min] }\end{array}$ & $\begin{array}{r}\text { Area \% } \\
{[\%]}\end{array}$ \\
\hline \hline 1 & 未知 & 6.23 & 84.77 & 579.3 & 90.2 & 84.765 \\
\hline 2 & 未知 & 7.65 & 15.23 & 84.1 & 16.2 & 15.235 \\
\hline & & & & & & \\
\hline Total & & & 100.00 & 663.3 & 106.5 & 100.000 \\
\hline
\end{tabular}




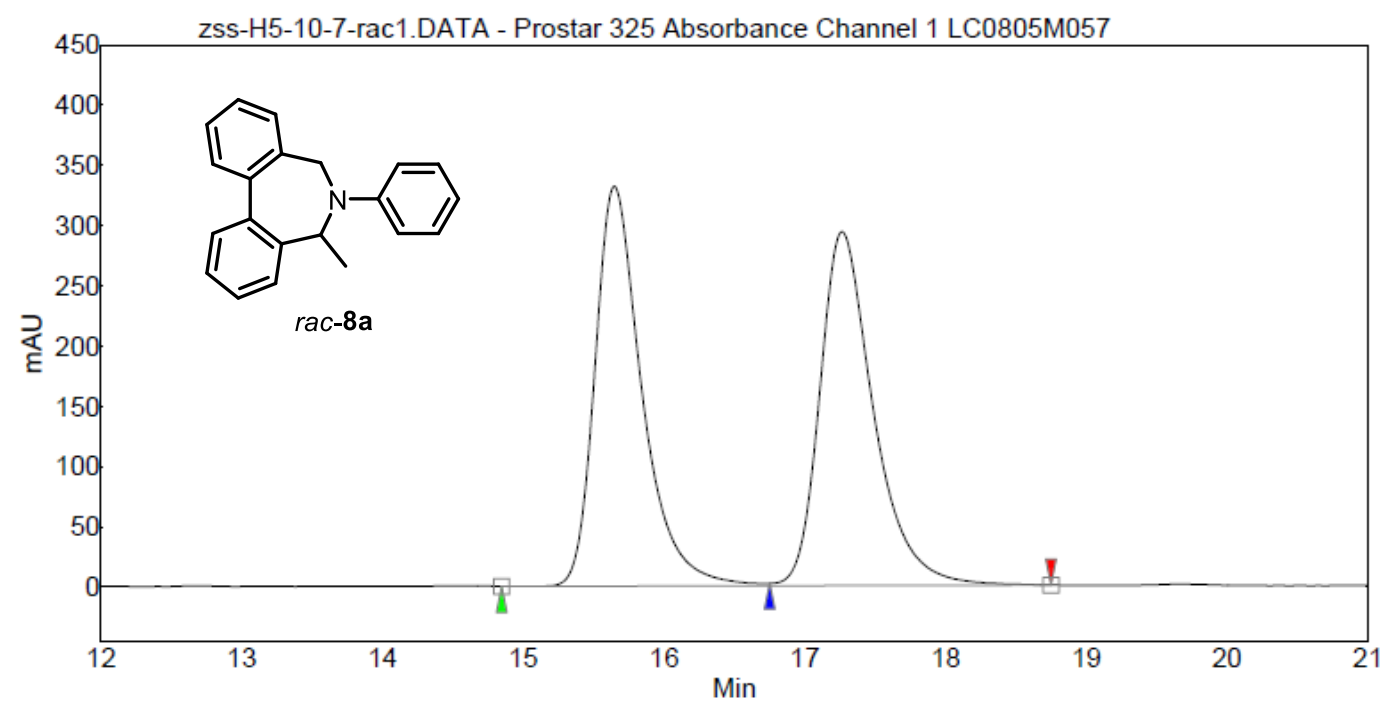

\begin{tabular}{|c|l|r|r|r|r|r|}
\hline Index & 文件名 & $\begin{array}{r}\text { 时间 } \\
\text { [Min] }\end{array}$ & $\begin{array}{r}\text { 数量 } \\
\text { [\% 面积] }\end{array}$ & $\begin{array}{r}\text { 高度 } \\
\text { [mAU] }\end{array}$ & $\begin{array}{r}\text { Area } \\
\text { [mAU.Min] }\end{array}$ & $\begin{array}{r}\text { Area \% } \\
\text { [\%] }\end{array}$ \\
\hline \hline 1 & 末知 & 15.65 & 49.73 & 331.8 & 128.3 & 49.732 \\
\hline 2 & 末知 & 17.26 & 50.27 & 293.6 & 129.7 & 50.268 \\
\hline & & & & & & \\
\hline Total & & & 100.00 & 625.5 & 257.9 & 100.000 \\
\hline
\end{tabular}

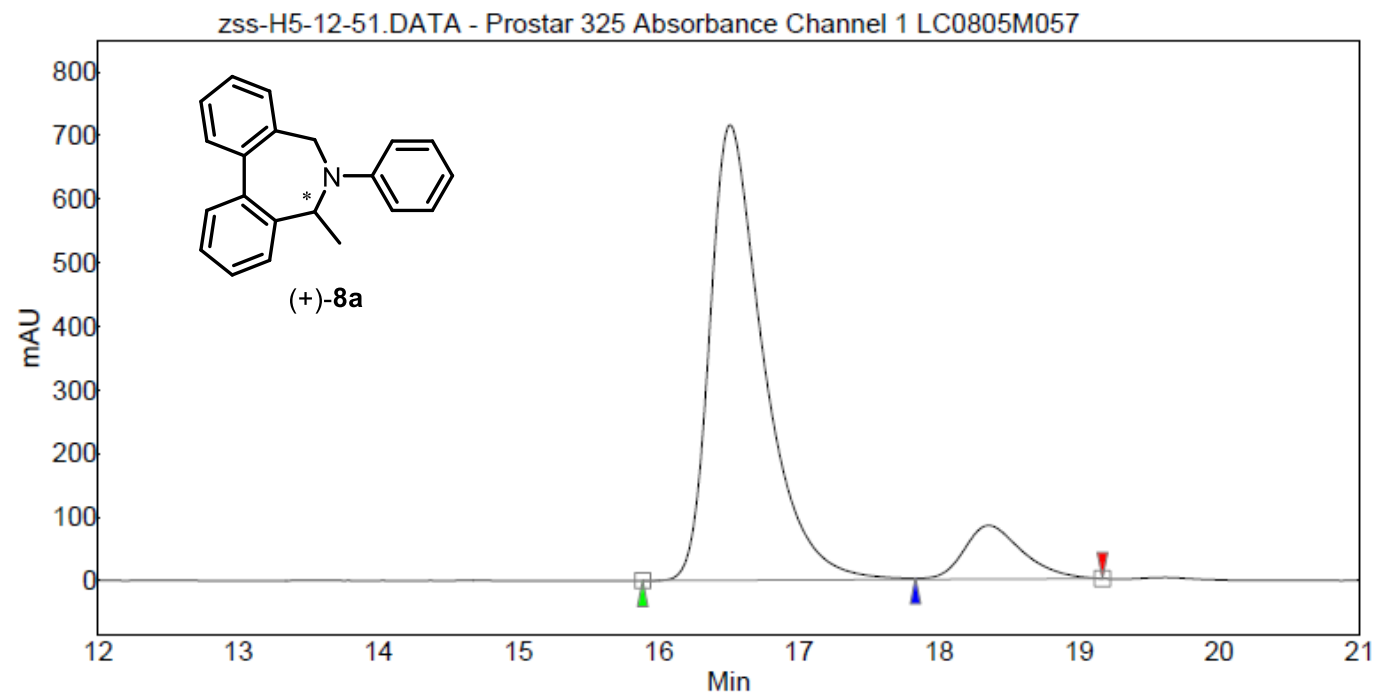

\begin{tabular}{|c|c|c|c|c|c|c|}
\hline Index & 文件名 & $\begin{array}{r}\begin{array}{l}\text { 时间 } \\
\text { [Min] }\end{array} \\
\end{array}$ & [\% 面积] & $\begin{array}{r}\text { 高度 } \\
\text { [mAU] }\end{array}$ & $\begin{array}{r}\text { Area } \\
\text { [mAU.Min] }\end{array}$ & $\begin{array}{r}\text { Area \% } \\
{[\%]}\end{array}$ \\
\hline 1 & 末知 & 16.51 & 88.66 & 715.9 & 317.6 & 88.656 \\
\hline 2 & 末知 & 18.35 & 11.34 & 84.4 & 40.6 & 11.344 \\
\hline Total & & & 100.00 & 800.3 & 358.2 & 100.000 \\
\hline
\end{tabular}




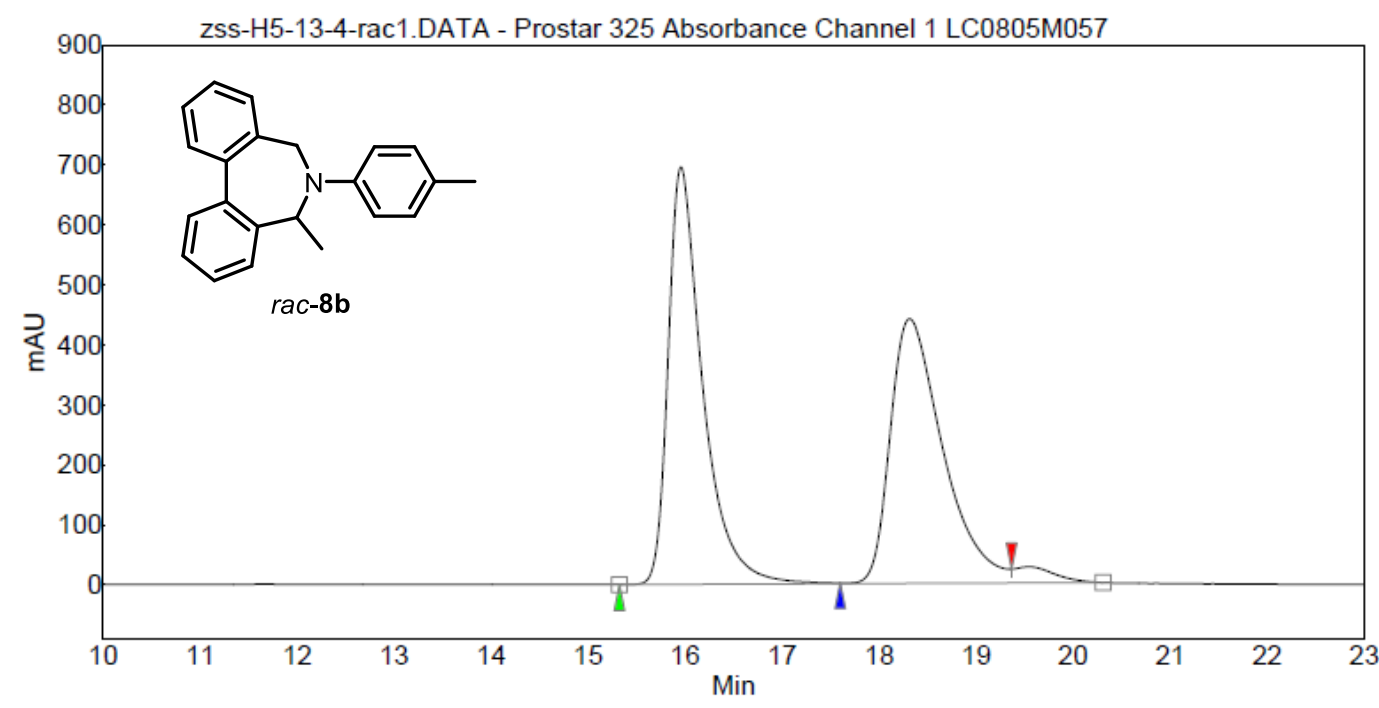

\begin{tabular}{|c|l|r|r|r|r|r|}
\hline Index & 文件名 & $\begin{array}{r}\text { 时间 } \\
{[\mathrm{Min}]}\end{array}$ & $\begin{array}{r}\text { 数量 } \\
\text { [\% 面积] }\end{array}$ & $\begin{array}{r}\text { 高度 } \\
{[\mathrm{mAU}]}\end{array}$ & $\begin{array}{r}\text { Area } \\
\text { [mAU.Min] }\end{array}$ & $\begin{array}{r}\text { Area \% } \\
\text { [\%] }\end{array}$ \\
\hline \hline 1 & 末知 & 15.95 & 50.93 & 695.6 & 287.2 & 50.928 \\
\hline 2 & 末知 & 18.31 & 49.07 & 441.0 & 276.7 & 49.072 \\
\hline & & & & & & \\
\hline Total & & & 100.00 & 1136.6 & 563.9 & 100.000 \\
\hline
\end{tabular}

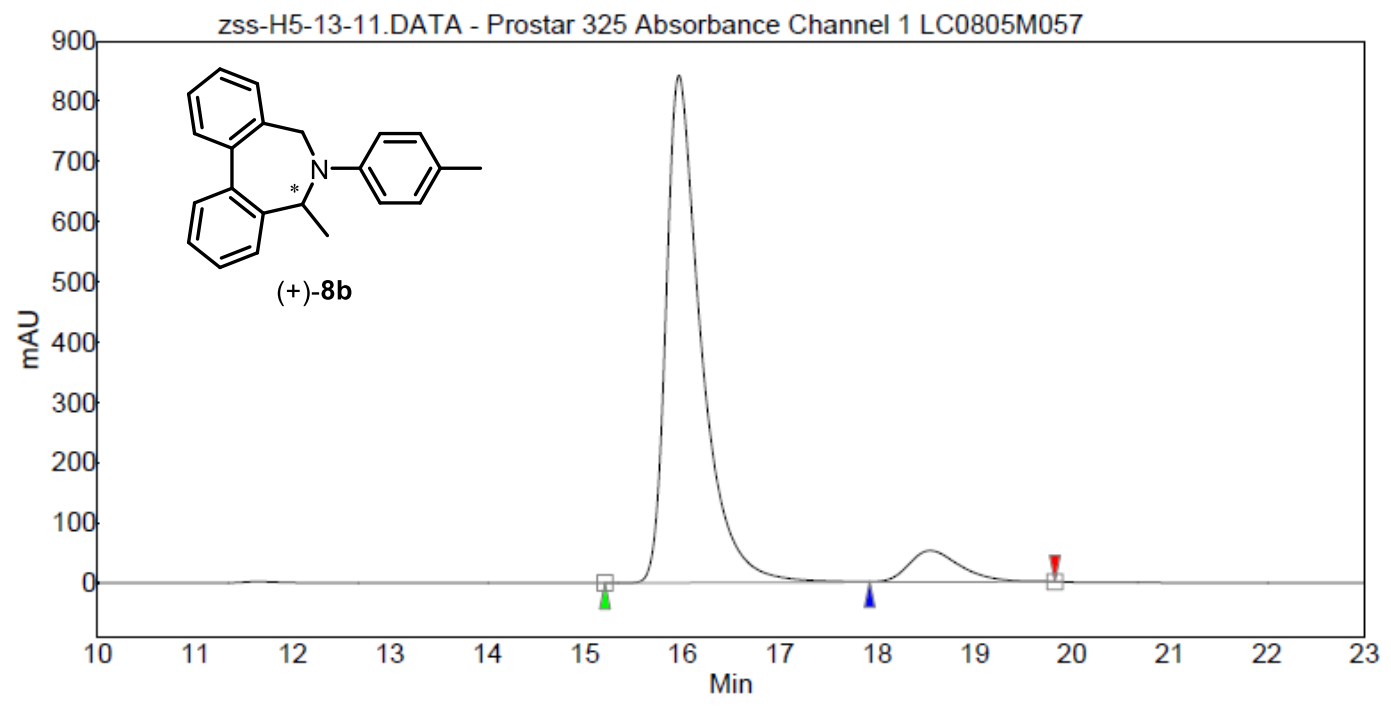

\begin{tabular}{|c|l|r|r|r|r|r|}
\hline Index & 文件名 & $\begin{array}{r}\text { 时间 } \\
\text { [Min] }\end{array}$ & $\begin{array}{r}\text { 数量 } \\
\text { [\% 面积] }\end{array}$ & $\begin{array}{r}\text { 高度 } \\
\text { [mAU] }\end{array}$ & $\begin{array}{r}\text { Area } \\
\text { [mAU.Min] }\end{array}$ & $\begin{array}{r}\text { Area \% } \\
\text { [\%] }\end{array}$ \\
\hline \hline 1 & 末知 & 15.97 & 91.62 & 842.8 & 351.9 & 91.616 \\
\hline 2 & 末知 & 18.55 & 8.38 & 52.1 & 32.2 & 8.384 \\
\hline & & & & & & \\
\hline Total & & & 100.00 & 894.9 & 384.1 & 100.000 \\
\hline
\end{tabular}




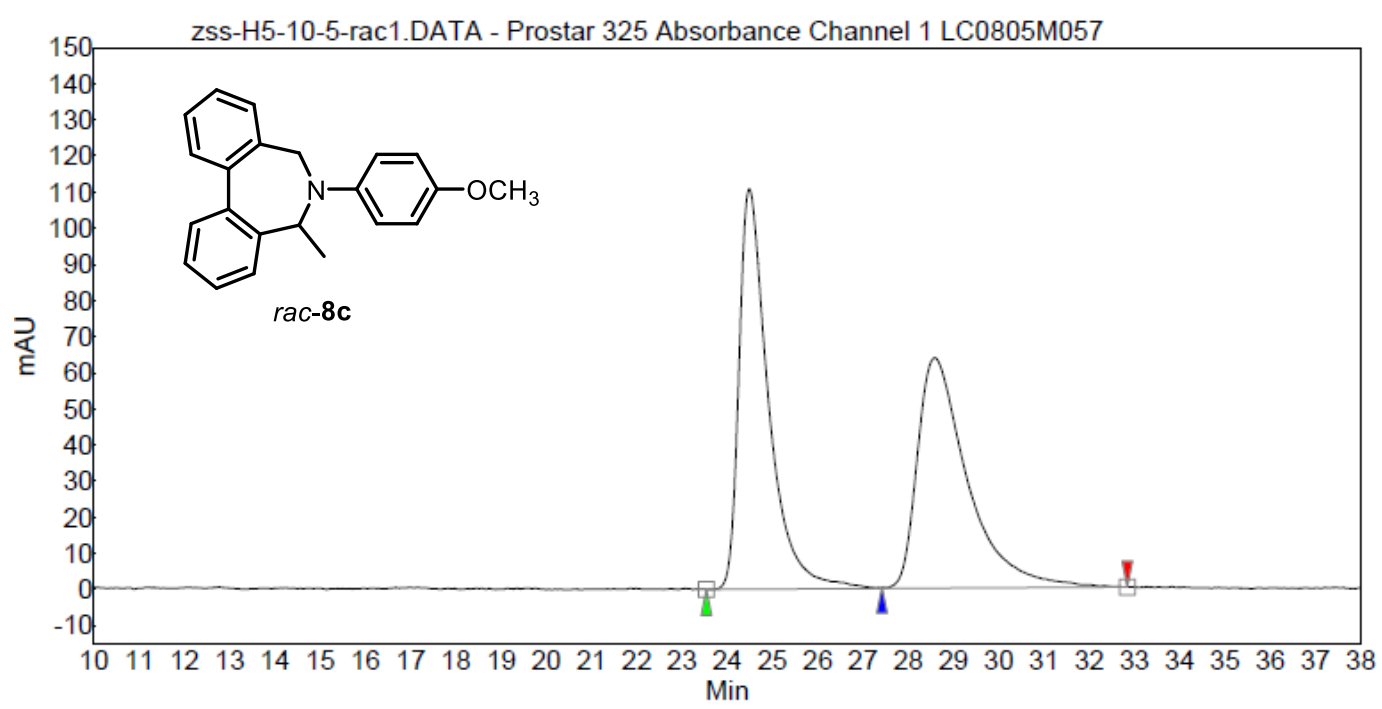

\begin{tabular}{|c|l|r|r|r|r|r|}
\hline Index & 文件名 & $\begin{array}{r}\text { 时间 } \\
\text { [Min] }\end{array}$ & $\begin{array}{r}\text { 数量 } \\
\text { [\% 面积] }\end{array}$ & $\begin{array}{r}\text { 高度 } \\
\text { [mAU] }\end{array}$ & $\begin{array}{r}\text { Area } \\
\text { [mAU.Min] }\end{array}$ & $\begin{array}{r}\text { Area \% } \\
\text { [\%] }\end{array}$ \\
\hline \hline 1 & 末知 & 24.48 & 50.59 & 110.8 & 83.9 & 50.586 \\
\hline 2 & 本知 & 28.58 & 49.41 & 63.7 & 81.9 & 49.414 \\
\hline & & & & & & \\
\hline Total & & & 100.00 & 174.5 & 165.8 & 100.000 \\
\hline
\end{tabular}

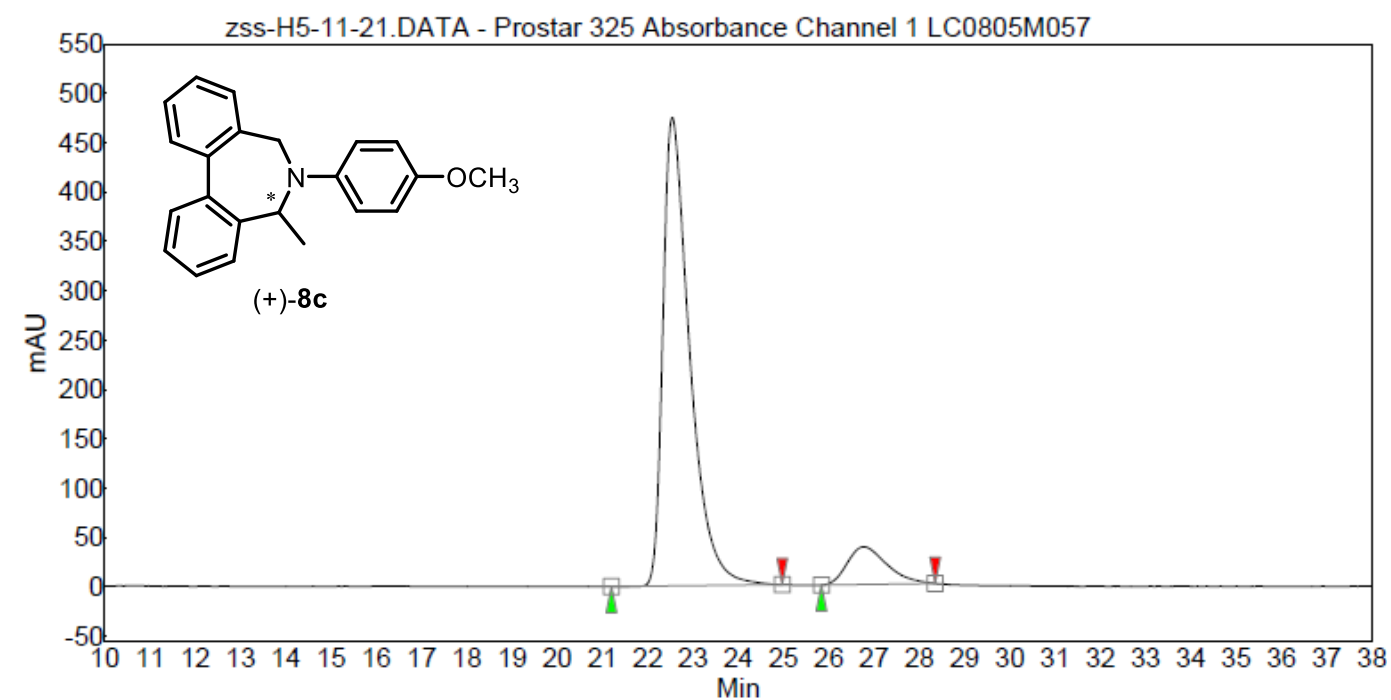

\begin{tabular}{|c|l|r|r|r|r|r|}
\hline Index & 文件名 & $\begin{array}{r}\text { 时间 } \\
{[\mathrm{Min}]}\end{array}$ & $\begin{array}{r}\text { 数量 } \\
\text { [\% 面积] }\end{array}$ & $\begin{array}{r}\text { 高度 } \\
{[\mathrm{mAU}]}\end{array}$ & $\begin{array}{r}\text { Area } \\
\text { [mAU.Min] }\end{array}$ & $\begin{array}{r}\text { Area \% } \\
{[\%]}\end{array}$ \\
\hline \hline 1 & 末知 & 22.53 & 89.38 & 474.5 & 325.4 & 89.379 \\
\hline 2 & 末知 & 26.77 & 10.62 & 38.2 & 38.7 & 10.621 \\
\hline & & & & & & \\
\hline Total & & & 100.00 & 512.6 & 364.1 & 100.000 \\
\hline
\end{tabular}




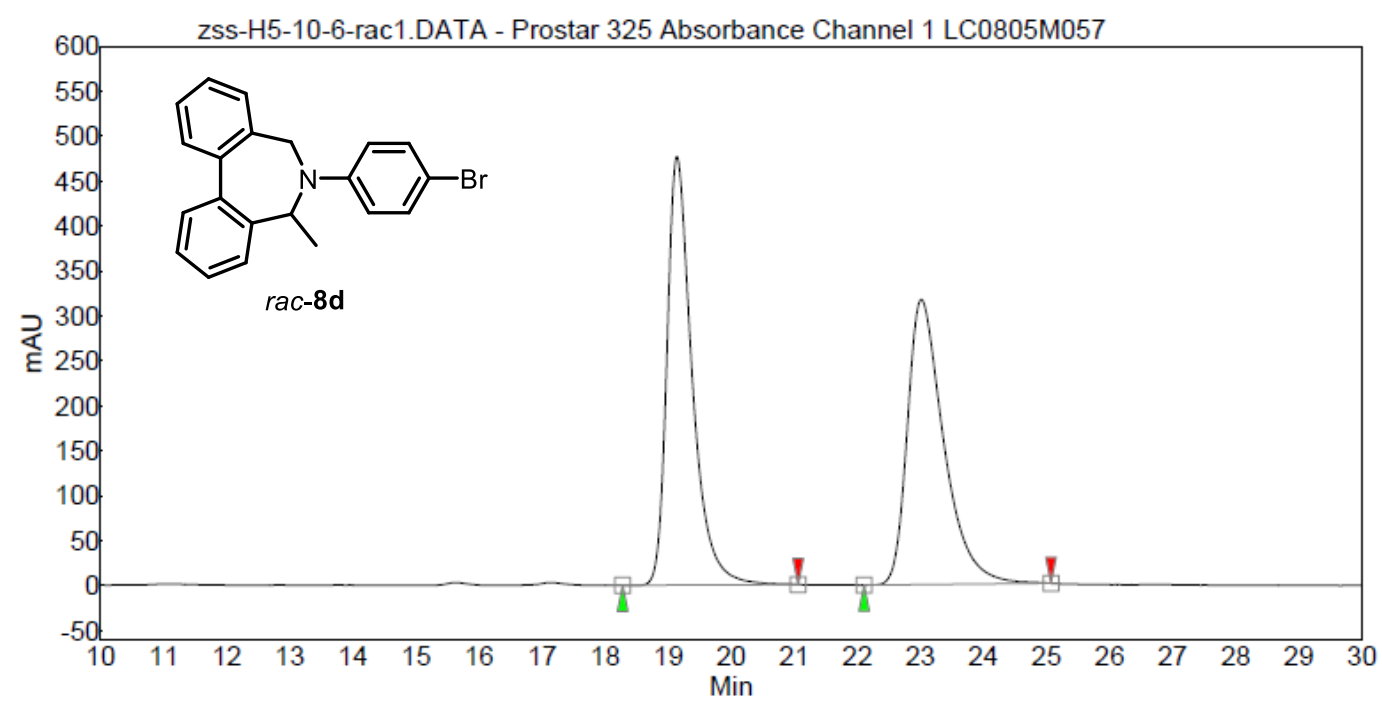

\begin{tabular}{|c|c|c|c|c|c|c|}
\hline Index & 文件名 & 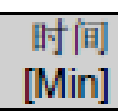 & [\% 面积] & $\begin{array}{r}\text { 高度 } \\
\text { [mAU] }\end{array}$ & $\begin{array}{r}\text { Area } \\
\text { [mAU.Min] }\end{array}$ & $\begin{array}{r}\text { Area \% } \\
{[\%]}\end{array}$ \\
\hline 1 & 本知 & 19.13 & 50.98 & 476.5 & 220.6 & 50.980 \\
\hline 2 & 本知 & 23.01 & 49.02 & 316.9 & 212.1 & 49.020 \\
\hline Total & & & 100.00 & 793.4 & 432.7 & 100.000 \\
\hline
\end{tabular}

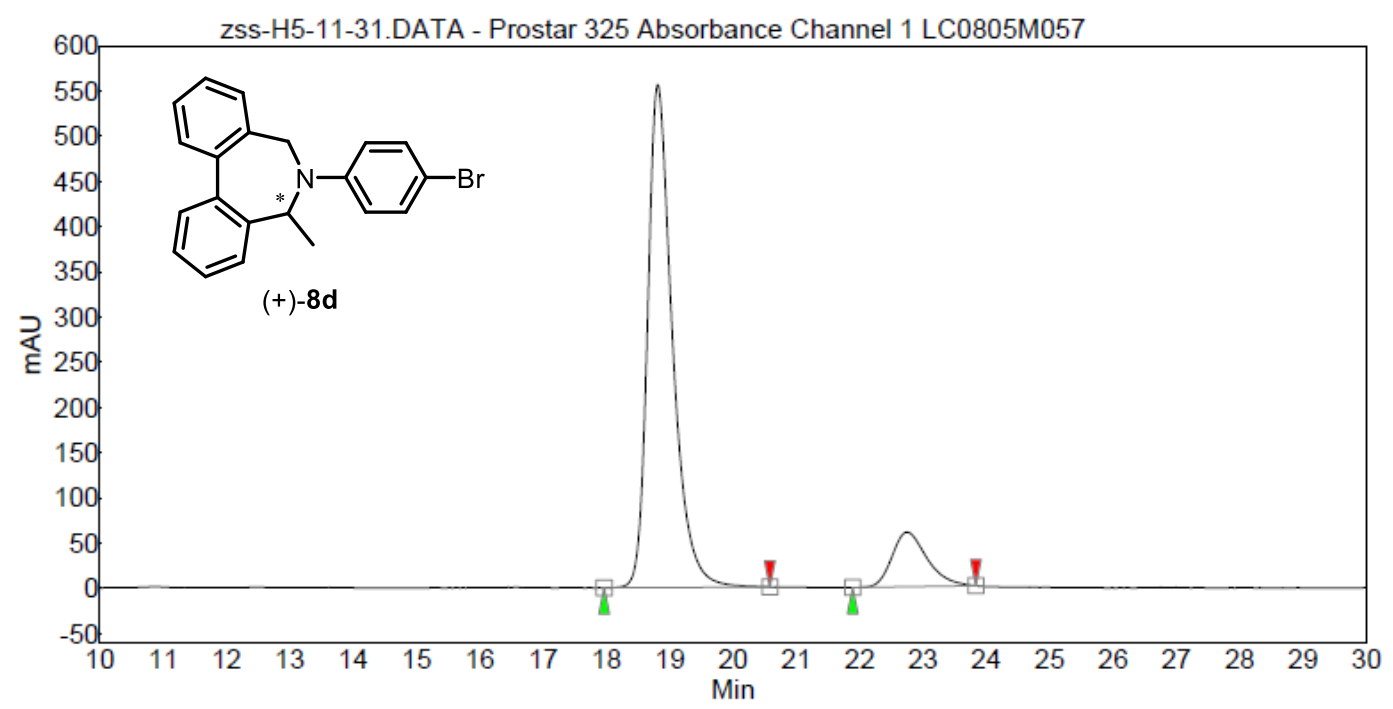

\begin{tabular}{|c|l|r|r|r|r|r|}
\hline Index & 文件名 & $\begin{array}{r}\text { 时间 } \\
\text { [Min] }\end{array}$ & $\begin{array}{r}\text { 数量 } \\
\text { [\% 面积] }\end{array}$ & $\begin{array}{r}\text { 高度 } \\
{[\mathrm{mAU}]}\end{array}$ & $\begin{array}{r}\text { Area } \\
\text { [mAU.Min] }\end{array}$ & $\begin{array}{r}\text { Area \% } \\
\text { [\%] }\end{array}$ \\
\hline \hline 1 & 末知 & 18.81 & 86.68 & 556.0 & 252.3 & 86.675 \\
\hline 2 & 末知 & 22.74 & 13.32 & 60.4 & 38.8 & 13.325 \\
\hline & & & & & & \\
\hline Total & & & 100.00 & 616.4 & 291.0 & 100.000 \\
\hline
\end{tabular}




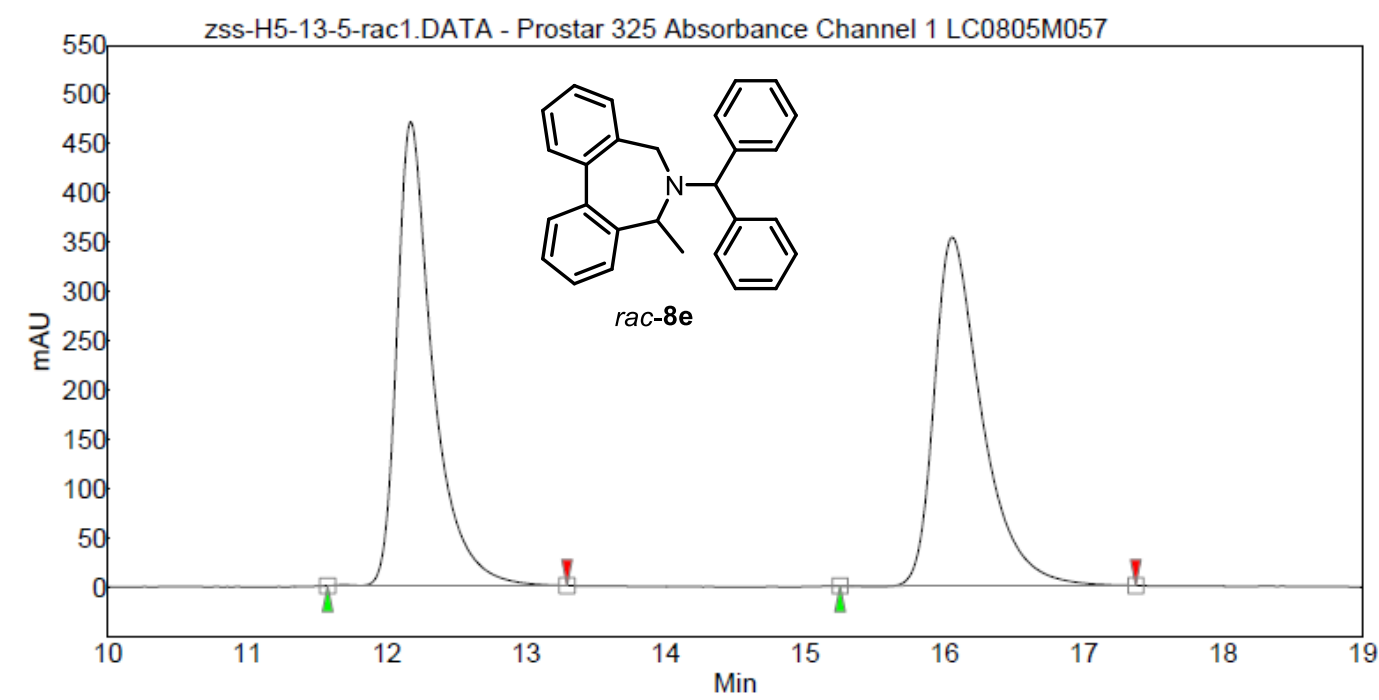

\begin{tabular}{|c|l|r|r|r|r|r|}
\hline Index & 文件名 & $\begin{array}{r}\text { 时间 } \\
\text { [Min] }\end{array}$ & $\begin{array}{r}\text { 数量 } \\
\text { [\% 面积] }\end{array}$ & \multicolumn{1}{|c|}{ [高度 } & $\begin{array}{r}\text { Area } \\
\text { [mAU] }\end{array}$ & $\begin{array}{r}\text { Area \% } \\
\text { [\%] }\end{array}$ \\
\hline \hline 1 & 末知 & 12.17 & 50.04 & 470.5 & 140.7 & 50.044 \\
\hline 2 & 本知 & 16.05 & 49.96 & 353.6 & 140.4 & 49.956 \\
\hline & & & & & & \\
\hline Total & & & 100.00 & 824.1 & 281.1 & 100.000 \\
\hline
\end{tabular}

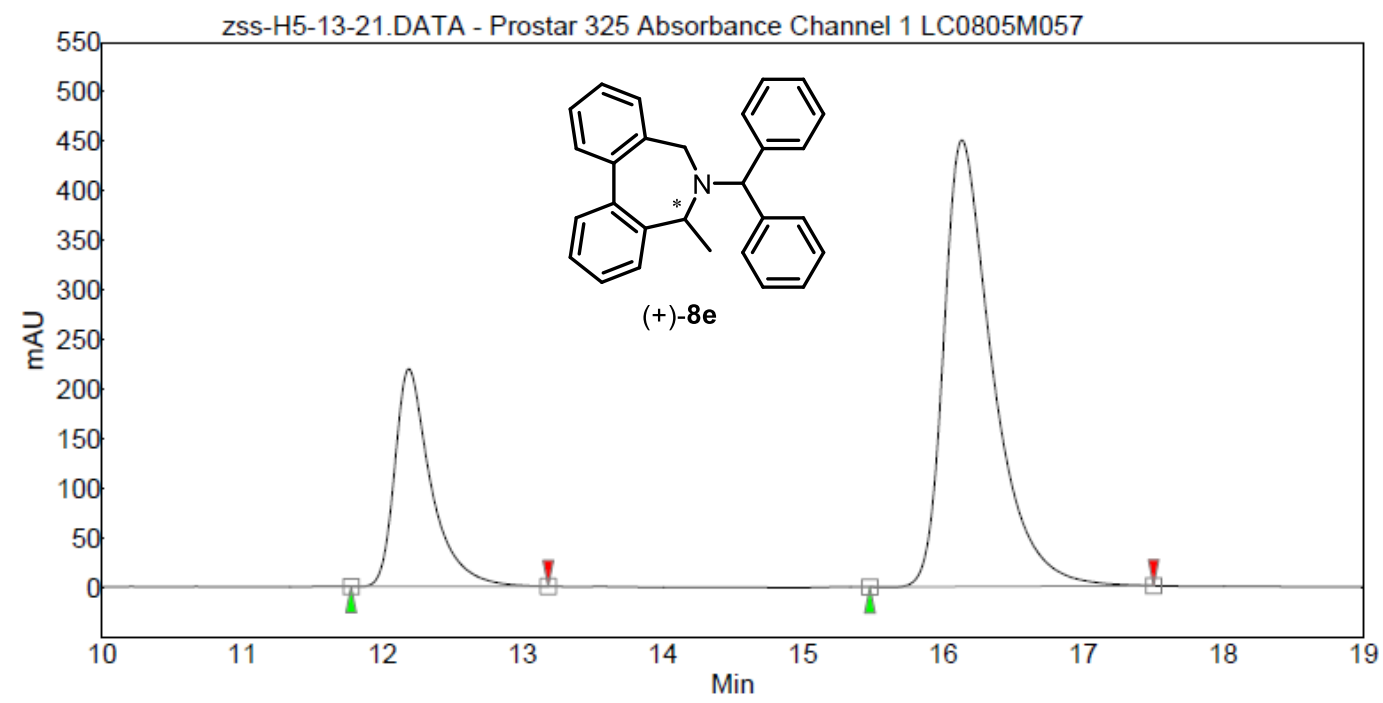

\begin{tabular}{|c|c|c|c|c|c|c|}
\hline Index & 文件名 & 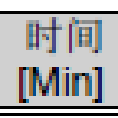 & [\% 面数量 & $\begin{array}{r}\text { 高度 } \\
\text { [mAU] }\end{array}$ & $\begin{array}{r}\text { Area } \\
\text { [mAU.Min] } \\
\end{array}$ & $\begin{array}{r}\text { Area } \% \\
{[\%]} \\
\end{array}$ \\
\hline 1 & 本知 & 12.19 & 26.63 & 219.5 & 66.0 & 26.627 \\
\hline 2 & 本知 & 16.13 & 73.37 & 450.4 & 182.0 & 73.373 \\
\hline Total & & & 100.00 & 669.8 & 248.0 & 100.000 \\
\hline
\end{tabular}

